BNWL-235 2

\title{
PACIFIC NORTHWEST LABORATORY ANNUAL REPORT FOR 1965 IN THE PHYSICAL SCIENCES
}

\author{
to the
}

USAEC Division of Biology and Medicine VOLUME 2: RADIOLOCICAL SCIENCES

MAY, 1966

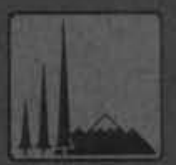

BattelleN=NoRthWest

BATTELLE MEMORIAL INSTITUTE / PACIFIC NORTHWEST LABORATORY 


\section{LEGAL NOTICE}

This report was prepared as an account of Government sponsored work. Neither the United States, nor the Commission, nor any person acting on behalf of the Commission:

A. Makes any warranty or representation, expressed or implied, with respect to the accuracy, completeness, or usefulness of the information contained in this report, or that the use of any information, apparatus, method, or process disclosed in this report may not infringe privately owned rights; or

B. Assumes any liabilities with respect to the use of, or for damages resulting from the use of any information, apparatus, method, or process disclosed in this report.

As used in the above, "person acting on behalf of the Commission" includes any employee or contractor of the Commission, or employee of such contractor, to the extent that such employee or confractor of the Commission, or employee of such contractor prepares, disseminates, or provides occess to, any information pursuant to his employment or contract with the Commission, or his employment with such contractor.

\section{PACIFIC NORTHWEST LABORATORY RICHLAND, WASHINGTON operated by BATTELLE MEMORIAL INSTITUTE}

for the

UNITED STATES ATOMIC ENERGY COMMISSION UNDER CONTRACT AT(45-1)-1830 


\section{BNWL-235 2 \\ $\mathrm{UC}-48$}

Biology and Medicine

PACIFIC NORTHWEST LABORATORY

ANNUAL REPORT FOR 1965

IN THE PHYSICAL SCIENCES

to the

USAEC Division of Biology and Medicine

VOLUME 2: RADIOLOGICAL SCIENCES

By

Staff Members of

Environmental and Radiological Sciences Department

Radiological Sciences Section

D. W. Pearce and J. K. Green

May, 1966

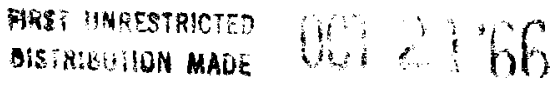

PACIFIC NORTHWEST LABORATORY

RICHLAND, WASHINGTON 
BNWL - 2352

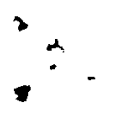

Printed in USA. Price $\$ 5.00$. Available from the Clearinghouse for Federal Scientific and Technical Information National Bureau of Standards U.S. Department of Commerce Springfield, Virginia 
TABLE OF CONTENTS

Page No.

\section{$\underline{\text { Radiological Physics }}$}

FAST PULSE AVERAGING-W. E. Wilson and W. C. Roesch . . . . . . . . . 1 TIME-RESOLVED SPECTROSCOPY-W. E. Wilson . . . . . . . . . . . . . . 4 SPECIFIC IONIZATION OF MONOENERGETIC PROTONS IN TISSUE-

EQUIVALENT GAS-D. N. Samsky and W. A. Glass . . . . . . . . . . 5 GRID-WALLED PROPORTIONAL COUNTER-W. A. Glass . . . . . • . . . . . 9 SELF ABSORPTION IN SCINTILLATION CRYSTALS-D. N. Samsky • • • . . .10 PULSED X-RAY MACHINE-L. A. Braby . . . . . . . . . . . . . . . . . .14 LOCAL ENERGY DENSITY AND HIT THEORY-W. C. Roesch . . . . . . . . . .15 LONG TERM CHANGE OF Pu-Be NEUTRON SOURCES-L. L. Nichols. . . . . . 18 LOCAL-ENERGY-DENSITY DISTRIBUTIONS-W. C. Roesch . . . . . . . . . . . . . . . CALORIMETRIC MEASUREMENT OF 210 Po-D. M. Fleming . . . . . . . . . . . 21 APPARATUS FOR LOCAL-ENERGY-DENSITY MEASUREMENTS--W. A GlasS . . . . .21 PROGRESS IN ABSORBED-DOSE-CALORIMETRY-D. M. Fleming . . . . . . . .25 FAST NEUTRON MEDICAL RESEARCH FACILITY-K. L. Swinth . . . . . . . . 26 TISSUE EQUIVALENT ION CHAMBER DEVELOPMENT-L. A. Braby. • • • • . . . 27 CALCULATION OF THE DISTRIBUTION OF DOSE IN LINEAR ENERGY

TRANSFER FROM SPHERICAL PROPORTIONAL COUNTER

DATA--D. N. Samsky and W. A. Glass . . . . . . . . . . . . . .29

LOW ENERGY FAST NEUTRON SPECTROMETER-W. A. Glass • • • • • • • • . . 33 TRANSITION ZONE DOSIMETRY-K. G. Pailthorp. . . . . . . . . . . . . 33 WHOLE BODY COUNTING OF PLUTONIUM IN DOGS-K. L. Swinth. . • . . . . . 39 SHORT-TERM RETENTION OF PLUTONIUM IN DOGS-K. L. Swinth . . . . . . .42 PROGRESS IN PLUTONIUM WHOLE BODY COUNTING-K. L. Swinth . . . . . . .45 ${ }^{55} \mathrm{Fe}$ IN THE MARINE ENVIRONMENT AND IN PEOPLE

WHO CONSUME OCEAN FISH-H. E. Palmer and T. M. Beasley . . . . .50 SEASONAL CYCLE OF ${ }^{137}$ CS IN ALASKAN NATIVES AT

ANAKTUVUK PASS-H. E. Palmer, W. C. Hanson and L. A. Braby . . . .54

THE APPLICATION OF SIMPLIFIED WHOLE BODY COUNTING IN ALASKA-H. E. Palmer . . . . . . . . . . . . . . . . . 55

Radiation Chemistry

DETERMINATION OF ERYTHROCYTE SIZE DISTRIBUTION-W. D. Felix • • . . .57 RADIATION-INDUCED HEMOLYSIS OF ERYTHROCYTES-W. D. Felix . . . . . .62 
TABLE OF CONTENTS (Contd)

$\underline{\text { Page No. }}$

EFFECT OF CHLORIDE ION ON THE RADIOLYSIS OF

ERIOGLAUCINE SOLUTIONS-W. D. Felix . . . . . . . . . . . . . . .64

ELECTRON SPIN RESONANCE DETECTION INSTRUMENTS-R. N. Diebel . . . 67

${ }^{60}$ CO IRRADIATOR FLOW SYSTEM FOR ELECTRON

SPIN RESONANCE-R. N. Diebel and D. R. Kalkwarf . . . . . . .70

LONG-LIVED ORGANIC RADICALS FORMED IN IRRADIATED

AQUEOUS SOLUTIONS-D. R. Kalkwarf and R. N. Diebel . . . . . . .71

REACTION KINETICS OF FREE RADICALS IN AQEOUS SOLUTION-D. R. Kalkwarf and R. N. Diebel . . . . . . . . . .76

CHEMICAL PROTECTION OF AGAROSE JELLIES FROM RADIATION DAMAGE - D. R. Kalkwarf . . . . . . . . . . . . 79

Radiological Chemistry

ALPHA-EMITTERS IN THE COLUMBIA RIVER-C. D. Jennings

and T. M. Beasley... . . . . . . . . . . . . . 82

THE REDUCTION OF REACTOR EFFLUENT WATER RADIONUCLIDE CONCENTRATIONS BY THE ADDITION OF SODIUM SILICATE

TO PROCESS WATER-D. E. Robertson . . . . . . . . . . . . .83

THE EFFECT OF REDUCED ALUM ADDITION ON THE REMOVAL OF $/ \mathrm{s}$ As

AT THE KW-REACTOR WATER TREATMENT PLANT-W. A. Haller. . . . . 88

ION EXCHANGE STUDIES OF THE CHEMICAL FORM OF RADIONUCLIDES

IN COLUMBIA RIVER WATER AND REACTOR EFFLUENT-Jack L. Nelson . . .89

DETERMINATION OF SEASONAL VARIATIONS IN SEDIMENT DEPOSITION

RATES IN MCNARY RESERVOIR OF THE COLUMBIA RIVER-Jack L. Nelson. .90

THE USE OF ${ }^{65} \mathrm{z} /{ }^{60}$ Co RATIOS TO DETERMINE AGE AND DEPOSITION

RATE OF RIVER BOTTOM SEDIMENTS-Jack L. Nelson and J. M. Nielsen .92

A METHOD FOR DETERMINATION OF ${ }^{32} \mathrm{P}$ AND OTHER RADIONUCLIDES

ON FILTERS CONTAINING SUSPENDED RIVER SEDIMENTS-Jack L. Nelson

and $\mathrm{W}$. B. Silker . . . . . . . . . . . . . .95

BEHAVIOR AND TRANSPORT OF RADIONUCLIDES IN THE COLUMBIA

RIVER BETWEEN HANFORD AND VANCOUVER, WASHINGTON-R. W. Perkins,

J. L. Nelson, and W. L. Haushild . . . . . . . . . . . .96

NATURAL AND ARTIFICIAL RADIONUCLIDE CONCENTRATION IN FARM PRODUCE IRRIGATED WITH COLUMBIA RIVER

WATER-R. W. Perkins . . . . . . . . . . . . 96

DISTRIBUTION AND EXCRETION OF TECHNETIUM IN HUMANS-T. M. Beasley,

H. E. Palmer and W. B. Nelp. . . . . . . . . . . 100

$210 \mathrm{~Pb}$ AND ${ }^{210} \mathrm{PO}$ IN ALASKAN BIOLOGICAL SAMPLES-T. M. Beasley and H. E. Palmer . . . . . . . . . . . . . . . 100

THE DISTRIBUTION OF NATURAL AND ARTIFICIAL RADIONUCLIDES

THROUGH THE FOOD CHAIN IN ALASKA-R. W. Perkins

and $\mathrm{W}$. C. Hanson .. . . . . . . . . . . . . 103 
TABLE OF CONTENTS (Contd)

Page No.

ULTRA SENSITIVE MEASUREMENT OF RADIONUCLIDES AND TRACE ELEMENTS CONSTITUENTS IN THE OCEAN BY MULTIDIMENSIONAL GAMMA-RAY SPECTROMETRY-R. W. Perkins, D. E. Robertson and H. G. Rieck. . . . . . . . . . . . . . . . . 108

COSMIC-RAY PRODUCED RADIONUCLIDES IN THE ENVIRONMENT-R. W. Perkins and J. M. Nielsen . . . . . . . . . 116

TRACE ELEMENT MEASUREMENTS IN SNOW-R. W. Perkins. . . . . . 116 CONCENTRATION OF FALLOUT RADIONUCLIDES DURING WET AND DRY SEASONS-C. W. Thomas. . . . . . . . . . . . . . . 117 AIRBORNE RADIONUCLIDES MEASUREMENT-R. W. Perkins . . . . . . . . 121 ${ }^{133}$ Xe AS AN AtMOSPheRIC TRACER-J. D. Ludwick . . . . . . . . 123 THE MEASUREMENT OF ${ }^{131}$ I TRANSPORT IN ATMOSPHERIC DIFFUSION EXPERIMENTS-J. D. Ludwick . . . . . . . . . . . 127

ORGANIC ${ }^{131}$ I COMPOUNDS RELEASED FROM A NUCLEAR FUEL CHEMICAL PROCESSING PLANT-W. H. Haller. . . . . . . . . . . . . 133

FISSION PRODUCT AEROSOL BEHAVIOR IN THE PLUTONIUM RECYCLE TEST REACTOR FUEL ROD FAILURE OF SEPTEMBER 29, 1965-R. W. Perkins, C. W. Thomas and W. B. Silker... . . . . . . . . . . . . . . 133

A SIMPLIFIED METHOD FOR THE DETERMINATION OF TRACE ELEMENTS IN GEOLOGIC SAMPLES BY ACTIVATION ANALYSIS-W. B. Silker . . . . . . . . . . . . . . . . . . . 134

IDENTIFICATION OF INDIVIDUAL BASALT FLOWS BY ACTIVATIONANALYSIS -W. B. Silker . . . . . . . . . . . . . . 135

TRACE ELEMENT IMPURITIES IN VITREOUS SILICA TUBING-D. E. Robertson . . . . . . . . . . . . . . . 137

PRELIMINARY EVALUATION OF AN EXISTING MULTIDIMENSIONAL GAMMA-RAY SPECTROMETER FOR WHOLE BODY COUNTING-D. E. Robertson and R. W. Perkins . . . . . . . . . . . . . . . . . . . 141

AN ANTICOINCIDENCE-SHIELDED Ge(Li) GAMMA-RAY SPECTROMETER-M. W. Hill . . . . . . . . . . . . . . . . . . 149

REDESIGN AND APPLICATION OF AN ANTICOINCIDENCE-SHIELDED Ge(Li) GAMMA-RAY SPECTROMETER-C. W. Thomas . . . . . . . . . 150

EVALUATION OF RADIOCHEMICAL METHODS FOR BIOLOGICAL PURPOSES-C. W. Thomas, W. B. Silker, and J. D. Ludwick . . . . 156

DETERMINATION OF ELEMENTS IN WHEAT GRAIN BY NEUTRON ACTIVATION ANALYSIS AND MULTIDIMENSIONAL GAMMA-RAY SPECTROMETRY-W. A. Ha11er . . . . . . . . . . . . . 156

SELECTIVE AND SENSITIVE ANALYSIS OF ACTIVATION PRODUCTS BY MULTIDIMENSIONAL GAMMA-RAY SPECTROMETRY -R. W. Perkins and D. E. Robertson . . . . 156 
TABLE OF CONTENTS (Contd)

Page No.

GAMMA-RAY BACKGROUND STUDIES FOR NaI(T1)

AND PlaStIC DETECTORS-C. W. Thomas . . . . . . . . . . 157

GAMMA-RAY TRANSITIONS IN ${ }^{58}$ Fe-M. W. Hill . . . . . . . . 162

TRACE ELEMENTS IN CANCEROUS AND NONCANCEROUS

HUMAN TISSUES - C. W. Thomas . . . . . . . . . . . . . . 164 
BNWL -2352

PACIFIC NORTHWEST LABORATORY

ANNUAL REPORT FOR 1965

IN THE PHYSICAL SCIENCES

\section{VOLUME 2: RADIOLOGICAL SCIENCES}

\section{RADIOLOGICAL PHYSICS}

FAST-PULSE AVERAGING-W. E. Wilson and W. C. Roesch

A block diagram of the system, an improvement of one reported earlier, (1) used to observe scintillations is presented in Figure 1. A signal is taken from the last dynode of the photomultiplier tube, amplified in wideband amplifiers, and then used to trigger the sampling scope. The anode signal is delayed to allow for the insertion delay of the trigger amplifiers and is analyzed without prior amplification.

An emitter-follower was inserted in the channel-advance line between the digital oscilloscope and the sampling oscilloscope. This then allows one to use this same pulse for counting the number of samples taken.

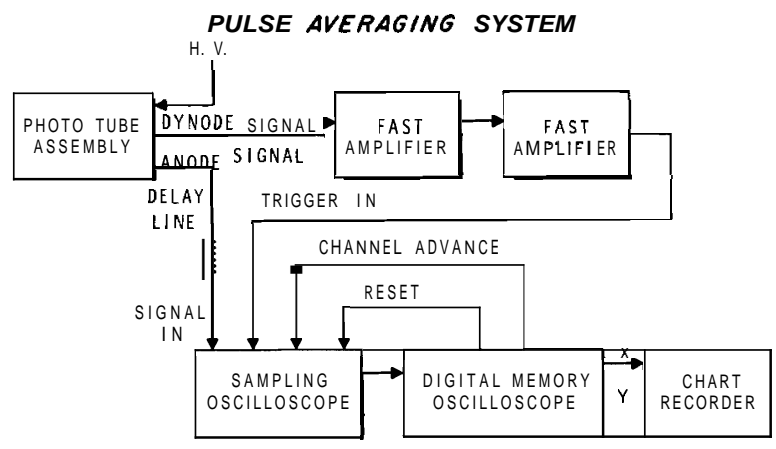

FIGURE 1. Pulse Averaging System
It was difficult to obtain a proper termination at the anode of the photomultiplier tube. This is very critical for observing the fast signals from stilbene, for example. The problem was largely solved by using a tube with a coaxial anode connection designed to match the input impedance $(50 \Omega)$ of the sampling oscilloscope. Figure 2 compares the averaged anode pulses obtained from a 56 AVP with an XP-1021. The reduction of circuit ringing achieved with the XP-1021 is obvious. The rise times and widths of these shapes agree with the manufacturer's specifications.

The averaged pulses from several common scintillators excited by ${ }^{60}$ Co were obtained and are presented in

Figures 3 through 6. The XP-1021 tube was used for all measurements but the $\mathrm{NaI}(\mathrm{T} 1)$, for which the 56 AVP was adequate. All crystals were thick ( $\geq 5 \mathrm{~mm}$ ), except again for the NaI(T1) which was very thin ( $0.025 \mathrm{~mm})$. The signal decay times-the time required for the photomultiplier tube response 


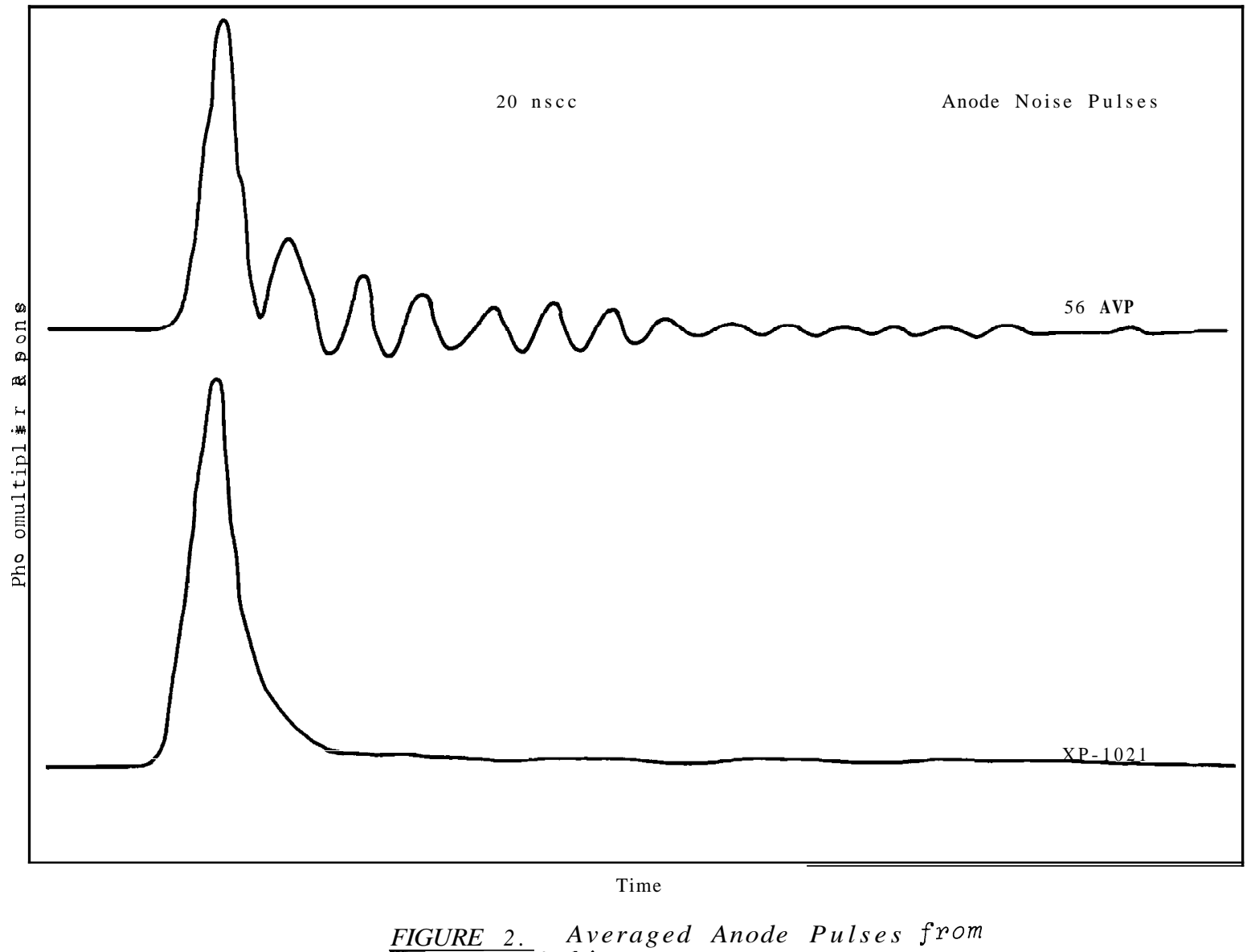

Photomultiplier Tubes

Anthracene

10 ns/cycle Calib.

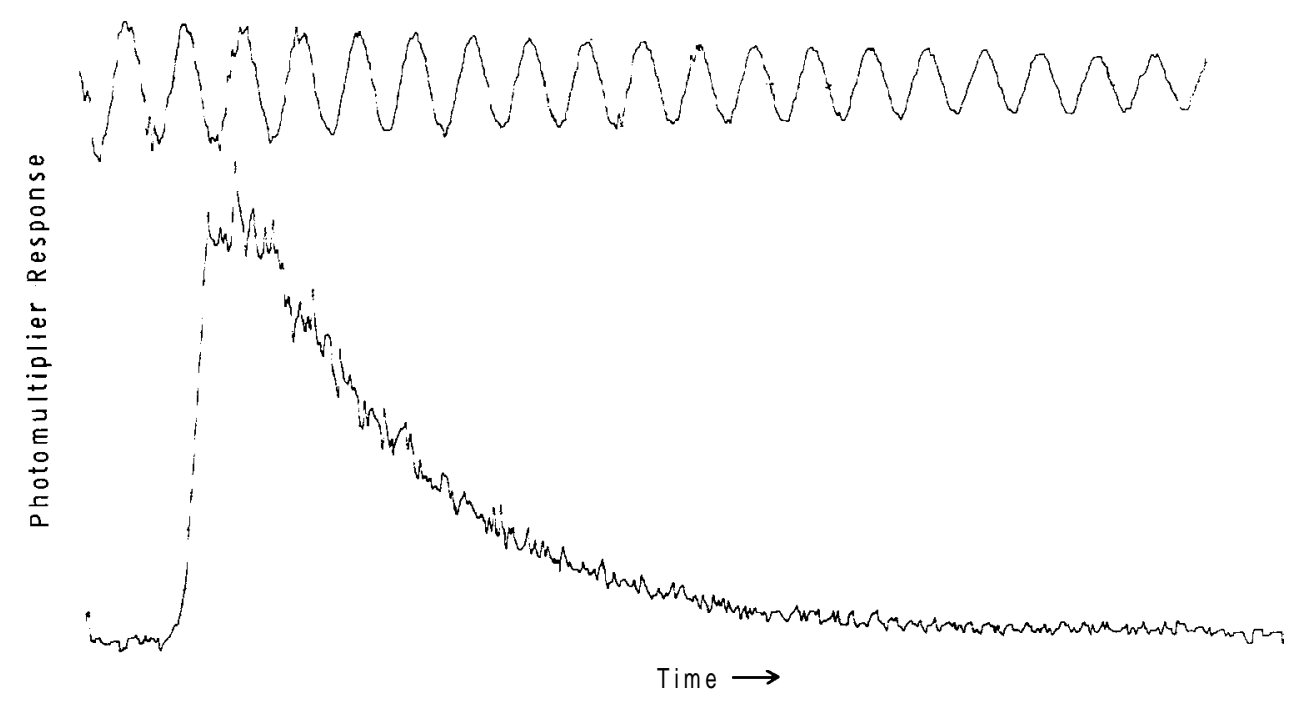

FIGURE 3. Scintizlation

$\overline{\text { Responses of Anthracene }}$ 

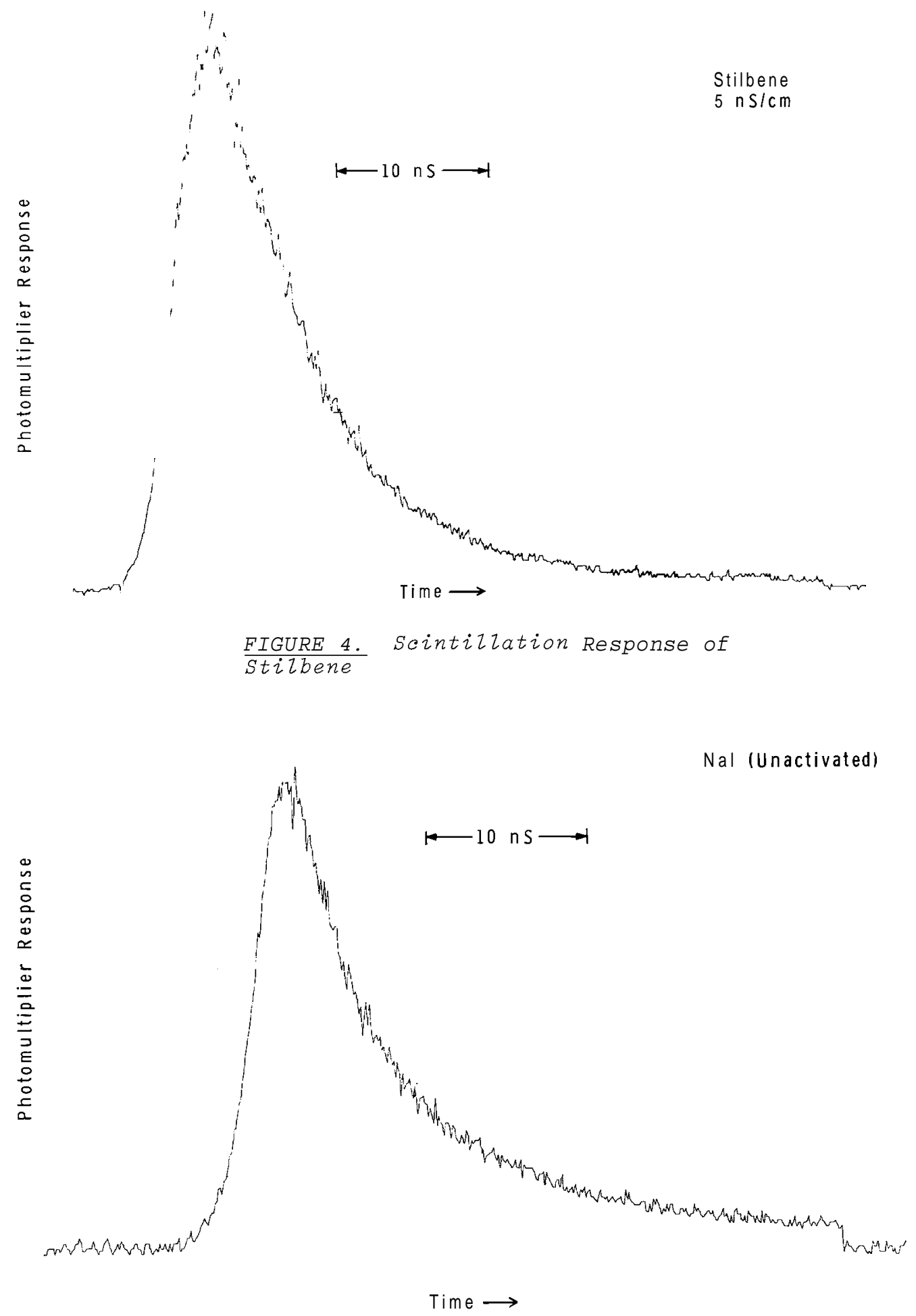

FIGURE 5. Scintiziation Response of $\mathrm{Na}$ NaI 


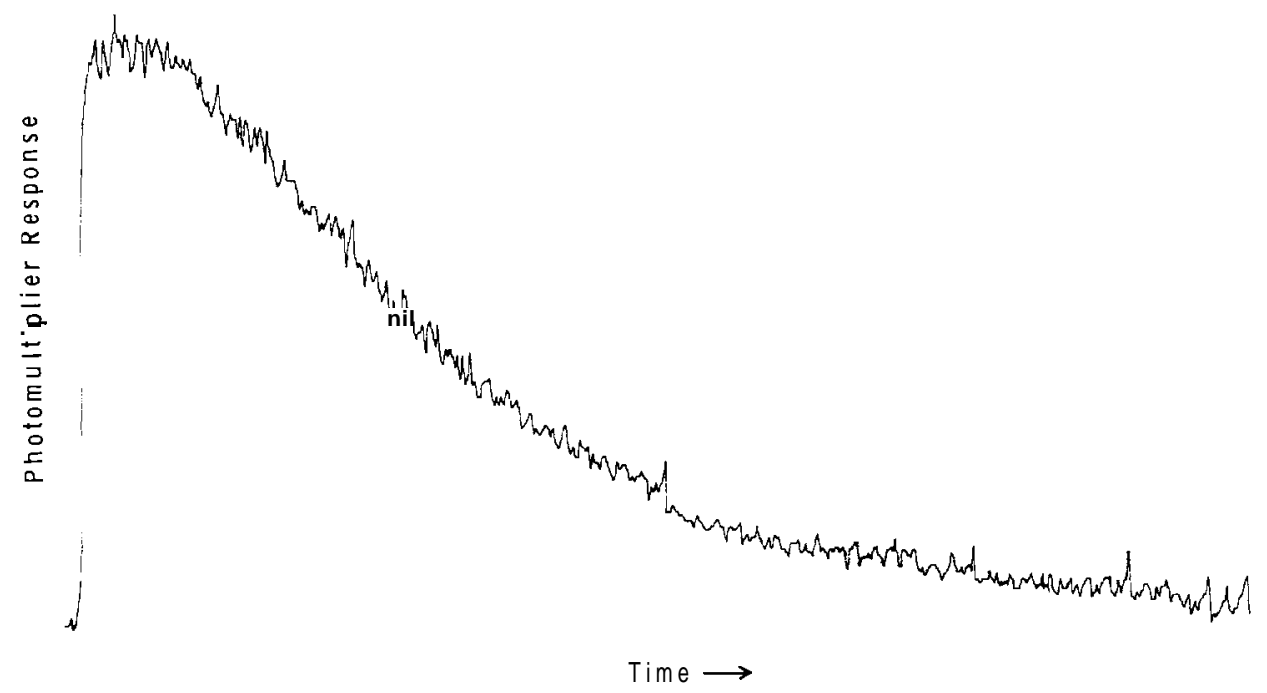

FIGURE 6. Scintizlation-

Response of Thallium Activa-

ted Sodium Iodide

to $e^{-1}$ of its initial value-observed directly with this system are given in Table I. Care must be used in interpreting these results for the two faster scintillators, because the photomultiplier-tube response function (Figure 2) is not negligible. If this response function is unfolded to obtain the natural decay time of the crystals, estimates consistent with other measurements are obtained.

TABLE 1. Signal Decay Times

\begin{tabular}{lc}
$\frac{\text { Crystal }}{\text { Anthracene }}$ & $\frac{\tau(\mathrm{nS})}{30}$ \\
Stilbene & 8.5 \\
$\mathrm{NaI}$ (Unactive) & 7 \\
$\mathrm{NaI}(\mathrm{T} 1)$ & 240 \\
\hline
\end{tabular}

Major components of the system have been acquired-a 500 mm Ebert-type scanning spectrometer, vacuum chamber for target holders, and various parts of the quartz optical system, and plans have been completed for a semipermanent beam port facility on the accelerator.

The pulse-averaging system ${ }^{(2)}$ was attached to the spectrometer as the detector and preliminary tests were made. Light emitted from a high-pressure xenon 
flash lamp was focussed on the entrance slit of the spectrometer and the spectrum recorded. The spectrum was a continuum through the region 400 $\mathrm{m}<\mathrm{A}<600 \mathrm{~m}(1 \mathrm{~mm} \equiv 10 \mathrm{~A})$ with several broad lines between 450 and $490 \mathrm{~nm}$ superimposed on it. To test the principle of the pulse-averaging system, the time dependence of two of these lines was measured. Figure 7 shows the emission at $472.9 \mathrm{~mm}$ and at $468.2 \mathrm{~mm}$ as a function of time. The time scale was not accurately calibrated, but the full width of the pulses is approximately $1.5 \mu \mathrm{sec}$. Within the accuracy of the measurements, the two lines decay with the same time dependence.

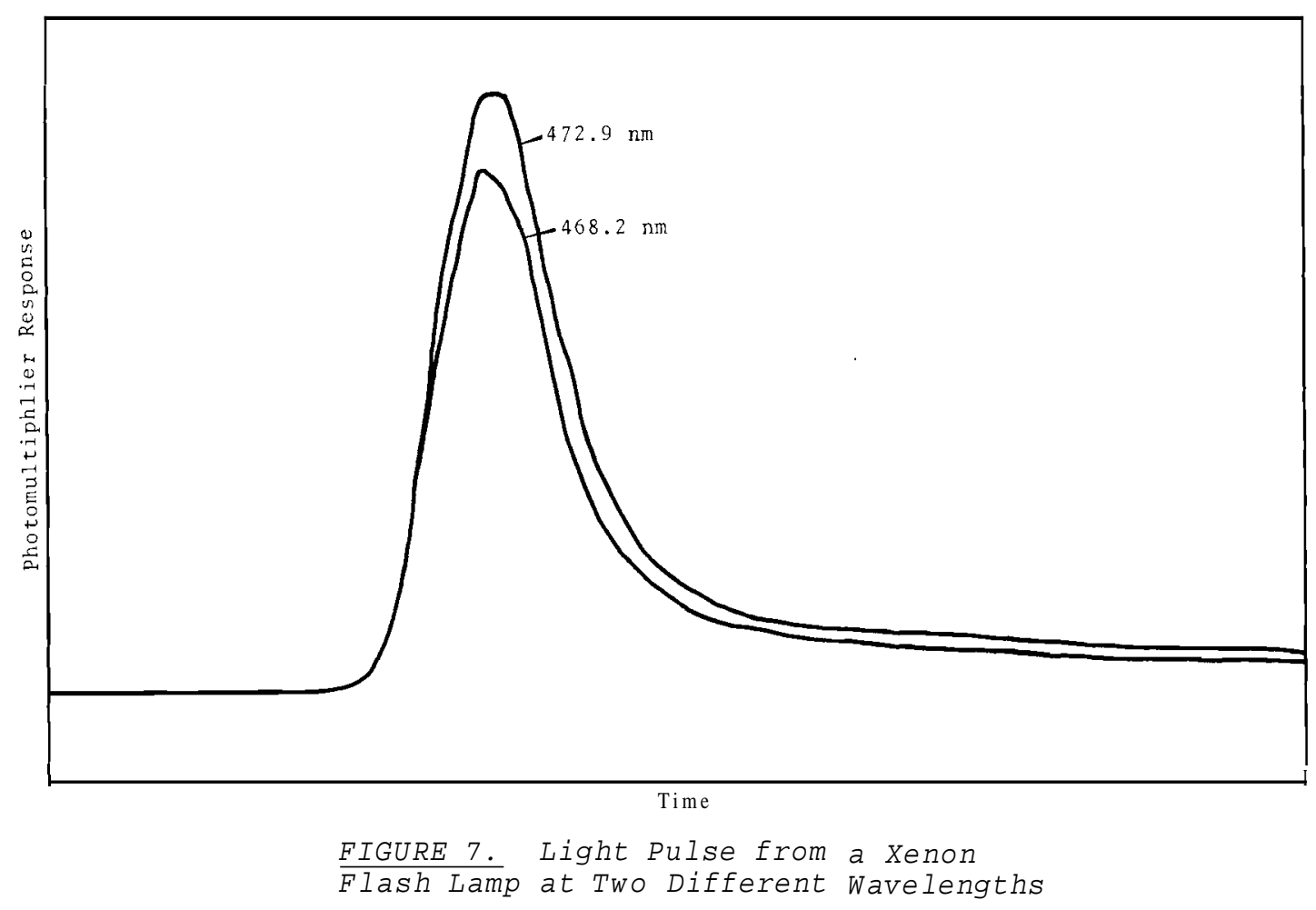

SPECIFIC IONIZATION OF MONOENERGETIC PROTONS IN TISSUE-EQUIVALENT GASD. N. Samsky and W. A. Glass

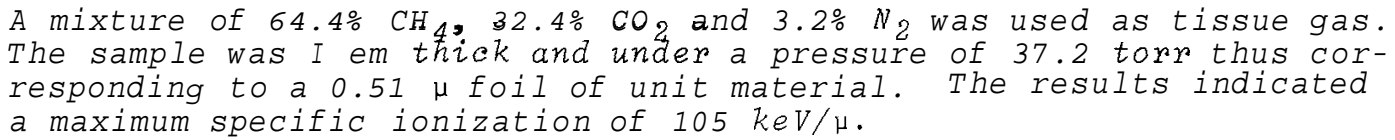

The study of the distribution of absorbed neutron dose in microscopic absorption sites has indicated the the need for more detailed information than is currently available regarding the specific ionization of protons in tissue-like materials.

Absorption sites of interest in neutron dosimetry are so small that the statistical nature of the specific ionization and the inferred energy loss becomes important. Due to the 
nature of ionization, the number of the ions formed in a given path length can vary. If the average number of ionizations is large, then the variation in that number can be approximated by a Gaussian distribution, and no distinction need be made between the average and the most probable values. Therefore, in keeping with the usual sense of specific ionization, average values will be used even in cases where the Gausian approximation no longer holds. Another common practice is the assignment of a constant value to the ratio of energy loss and ionization. Although this procedure is not defensible for proton energies below the onset of charge exchange, the specific ionization will be reported in terms of $\mathrm{keV} / \mu$. It must be stressed, however, that for low energy protons, the specific ionization is not a good indication of energy loss. Further, a distinction must be made between the energy lost by the proton and that gained by the gas. Proton energy lost through coulomb interaction will occasionally produce electrons with sufficient energy to contribute to the observed ionization. These $\delta$-rays may contribute to the measured ionization even though the proton lost the energy outside the region of interest. This experiment was designed to measure the energy transferred to the medium under charged particle equilibrium conditions; that is, the protons were required to pass through sufficient gas to establish equilibrium with the most energetic 6-rays.

The specific ionization measurements were made with a thin-foil proportiona 1 counter; the construction details are shown in Figure 8. The thin foil is defined by a $1 \mathrm{~cm}$ window in a shield tube that surrounds the proportional counter anode. The voltage on the tube was adjusted to match the equipotential surface in the cylindrical

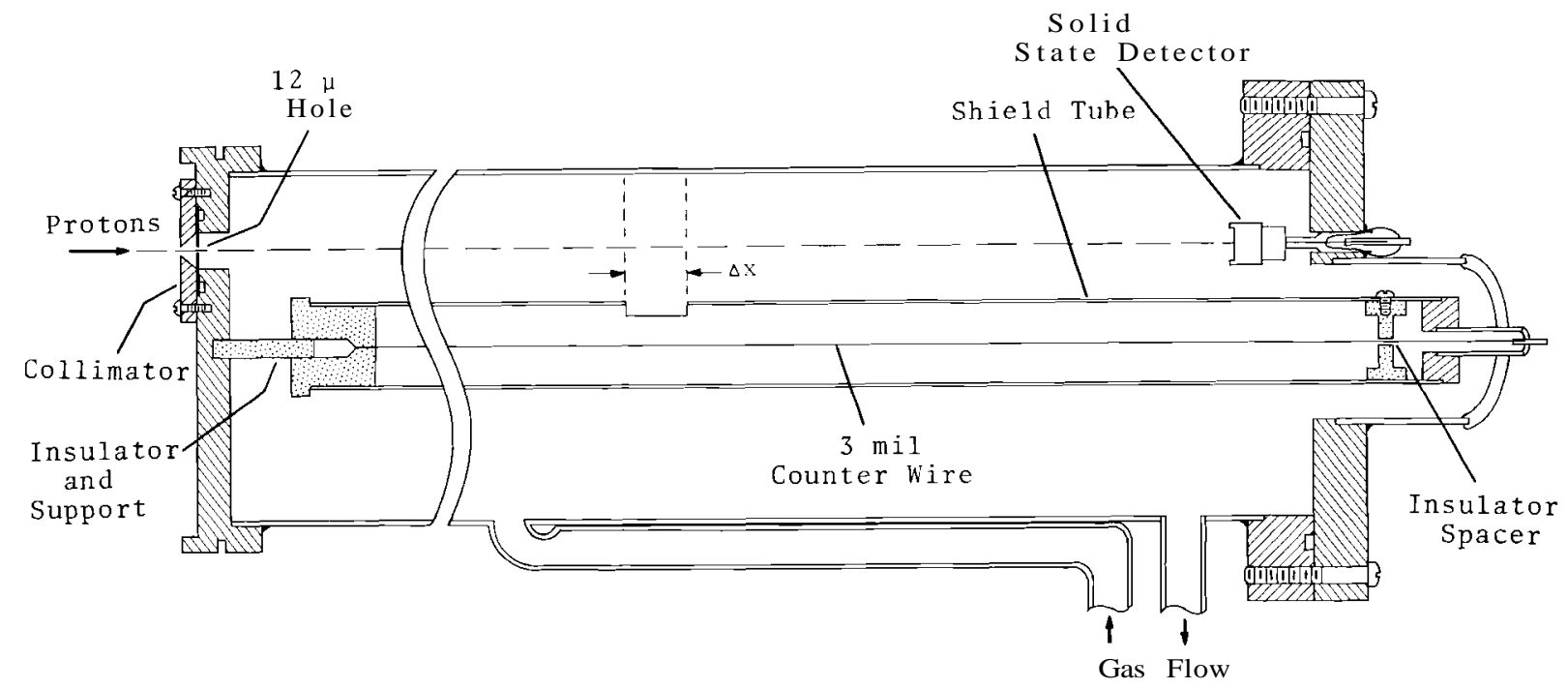

FIGURE 8. The Thin Foil Proportional Counter 
counter action was not disturbed, and the region of ion collection was well defined.

The proton beam of the Van de Graaff accelerator was magnetically analyzed and collimated by a $12 \mu$ diam hole before entering the chamber. Except at very low energies, the protons traversed the entire length of the chamber and stopped in a solid state detector. The responses of the latter with various gas pressures in the chamber were used to calibrate the proportional counter. Signals from this detector were also used to gate the signals from the proportional counter, thus providing additional collimation. The apparatus was operated under constant gas flow conditions at a pressure of $\mathbf{3 7 . 2}$ torr. At this pressure and at $20{ }^{\circ} \mathrm{C}$, the $1 \mathrm{~cm}$ sample of gas represents a unit density foil of $0.51 \mu$.

A block diagram of the electronic system is shown in Figure 9. The charge-sensitive preamplifier was chosen to be compatible with the action of the proportional counter. The discriminator was set to accept for analysis only those protons that passed through the foil parallel with the anode wire.

The data for this experiment were taken as a function of proton energy before they entered the chamber. Figure 10 shows the uncalibrated response of the proportional counter at 37.2 torr and responses of the detector at 37.2 torr, at calibration pressure $\left(\mathrm{P}_{\mathrm{C}}\right)$, and at 0.0 torr. The calibration pressure, approximately 19 torr, is that pressure which simulates the positioning of the detector at the front edge of the sensitive volume at the operating pressure. For machine energies above about $1.0 \mathrm{MeV}$, the slope of the proportional counter is nearly constant, and an energy calibration of it can be made. The average energy lost in the sensitive volume can be taken as the ratio of the length of the sensitive volume to the length of the chamber, times the energy lost in the chamber as measured by the detector. The result, a calibrated response curve of the proportional counter, is shown in Figure 11. The peak of the ionization curve occurs at $100 \mathrm{keV}$ and reaches a maximum of $105 \mathrm{keV} / \mu$.

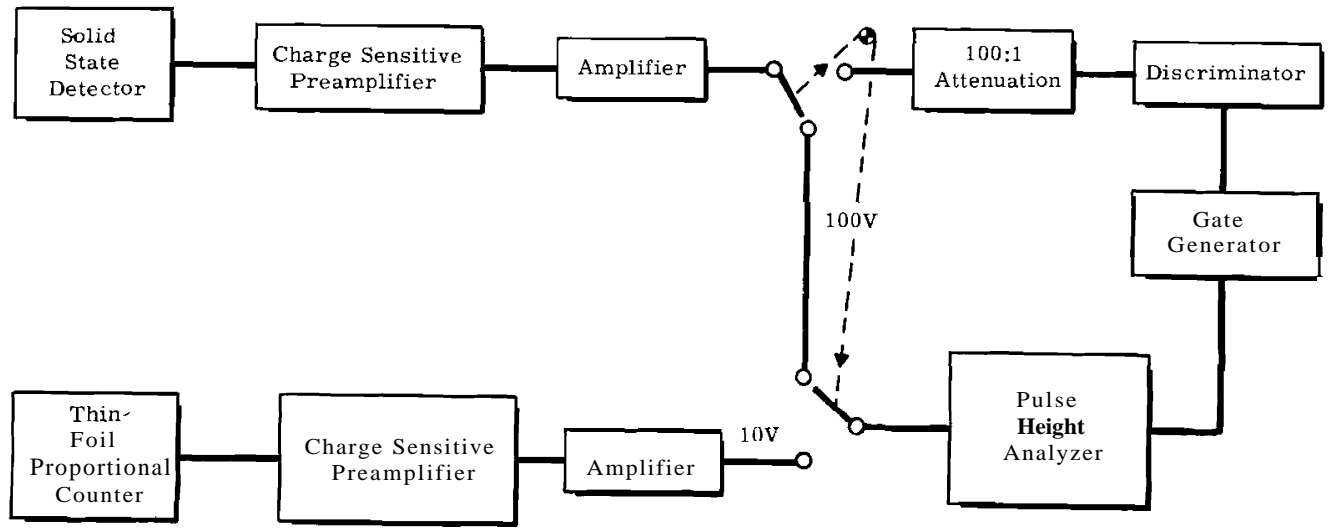

FIGURE 9. The Electronic System for the Thin Foil Proportional Counter 


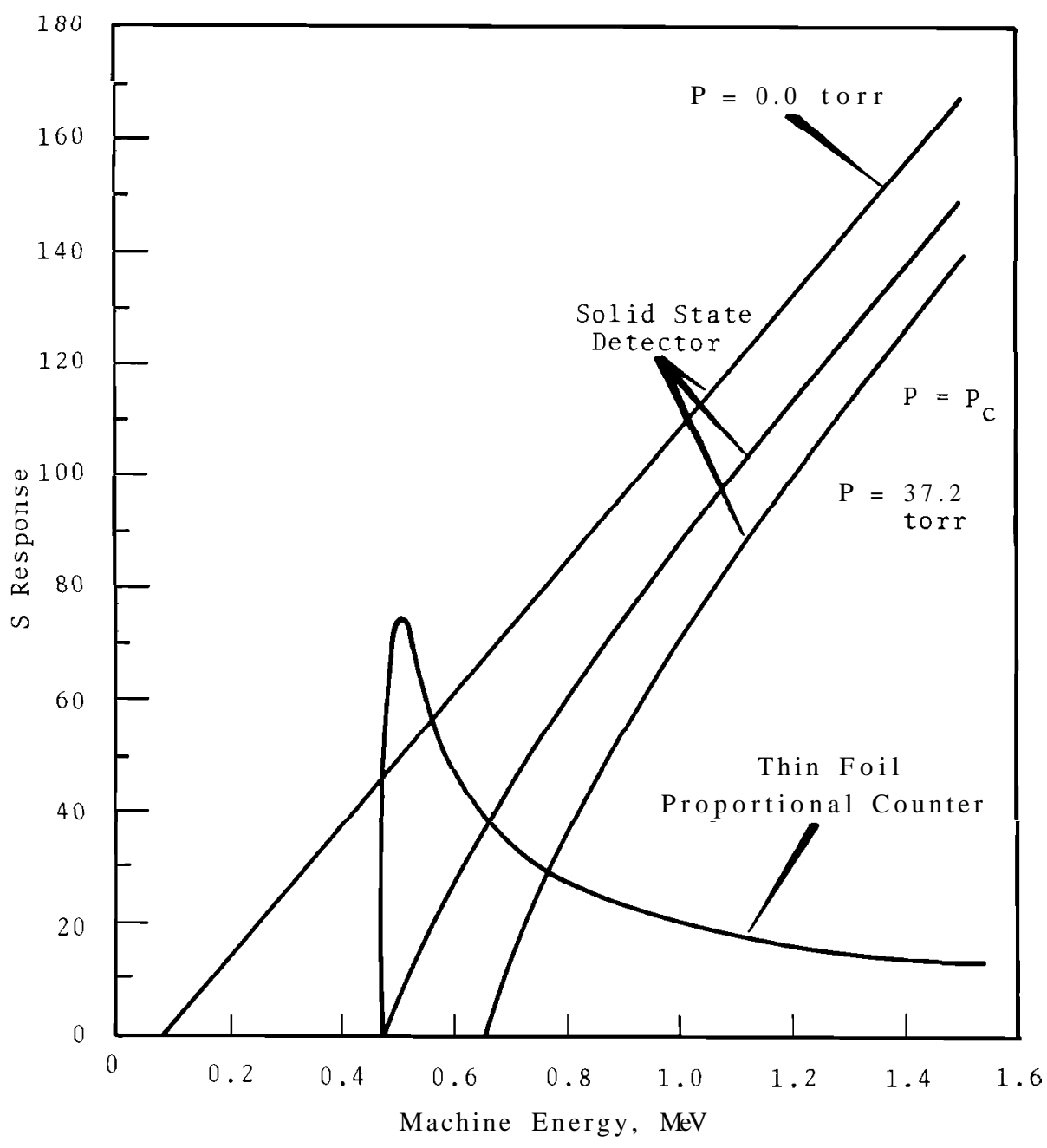

FIGURE 10. Response of the

Solid State Detector and

the Thin Foil Proportional

Counter

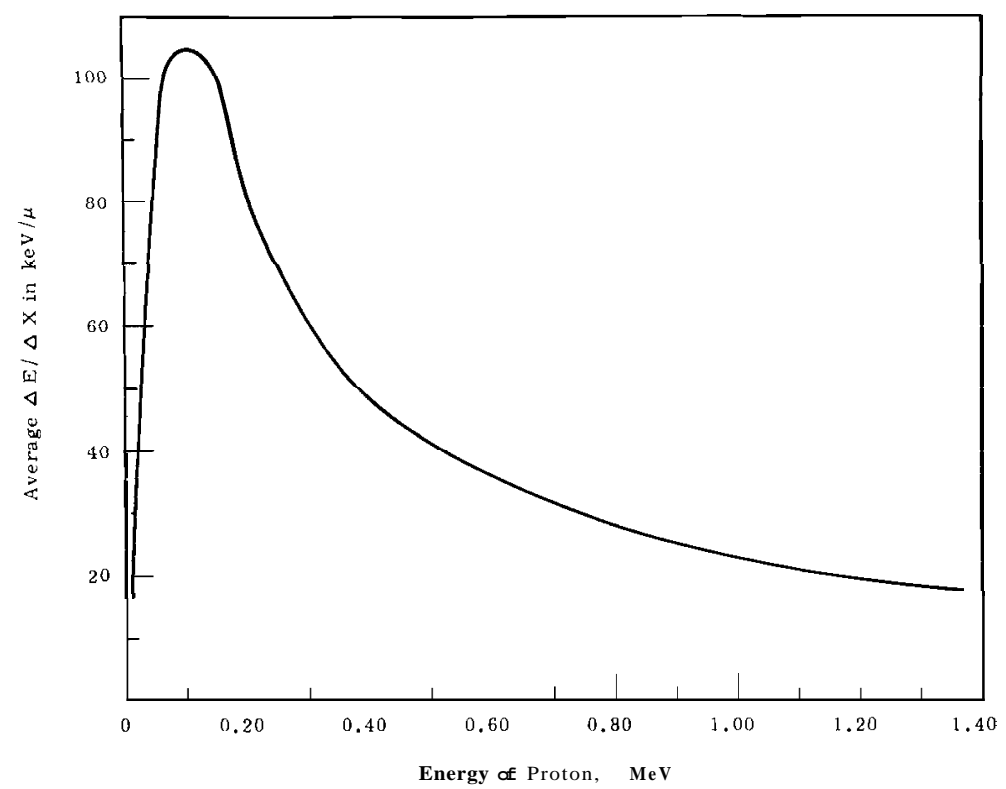

FIGURE 11. $\triangle E / \triangle X$ of Protons

in a Tissue Equivalent Gas

Sample Corresponding to a Unit

Density Foil of $0.51 \mu$ 
GRID-WALLED PROPORTIONAL COUNTER-W. A. GI as S

\begin{abstract}
A miniature proportional counter was developed for studies of the effect of wall-scattered radiation on measured local-energy-density distributions. It has a helical-grid cathode structure that is approximately transparent. The sensitive volume is a cylinder $1 \mathrm{~cm}$ diam and $1 \mathrm{~cm}$ long.
\end{abstract}

Proportional counters make possible the measurement of the distribution of energy deposited in microscopic sites by ionizing radiation. It is usually assumed that the distribution of energies deposited in such a counter by ionizing radiation is the same as that in the equivalent microscopic site.

Rossi (3) has given an example that shows that the assumption is not rigorously true. A particle, particularly an electron, may cross the counter, undergo multiple scattering in the wall, and come back through the counter; whereas, a similar electron going through the equivalent solid would have missed the volume after being scattered.

To investigate just how much this phenomenon influences the energy distributions measured, it was decided to compare distributions measured with a conventional solid-wall counter with

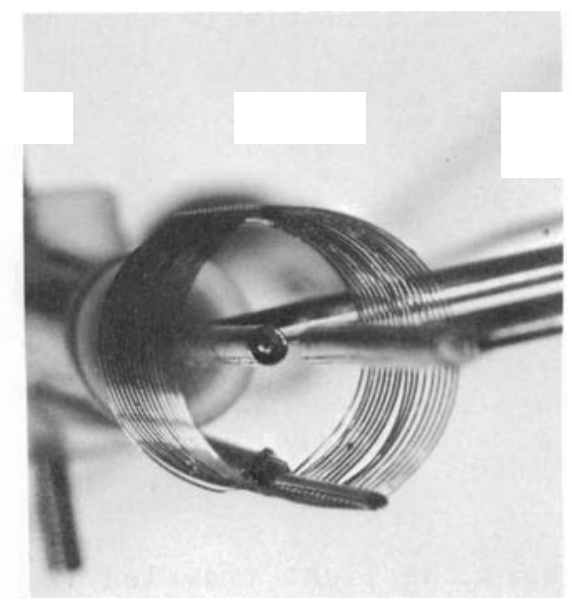

FIGURE 12. One Centimeter Diameter Grid-WaZZed Counter with Fiezd Shaping Electrodes those measured in a counter of the same size but whose walls were of the same density as the gas in the counter. Scattering in the latter should be similar to that in the equivalent solid.

A counter with essentially transparent walls would be placed in a very large tank that contained the counting gas. The investigation would be made with a beta-ray emitter dispersed in the gas and whose range was less than the distance from the counter to the wall of the tank. This would simulate a system of uniform density in which there was charged particle equilibrium. The similar situation will be simulated in the conventional counter by incorporating the same beta-emitter in its walls and counting gas.

The counter has been completed and tested and is illustrated in Figure 12. It is a circular cylinder $1 \mathrm{~cm}$ diam and $1 \mathrm{~cm}$ long. The transparent wall is made of $0.127 \mathrm{~mm}$ wire wound as a helix with spacing of $1.6 \mathrm{~mm}$ between adjacent turns. This construction provides greater than $90 \%$ transparency to incident beta particles, yet provides a satisfactory cathode and definition of the counting volume for the cylindrical counter. The helix is supported by two wires running the length of the structure. These also provide support for a solid wall that can be added without disturbing the internal parts of the counter to make the corresponding conventional counter Since the length of the counter is 


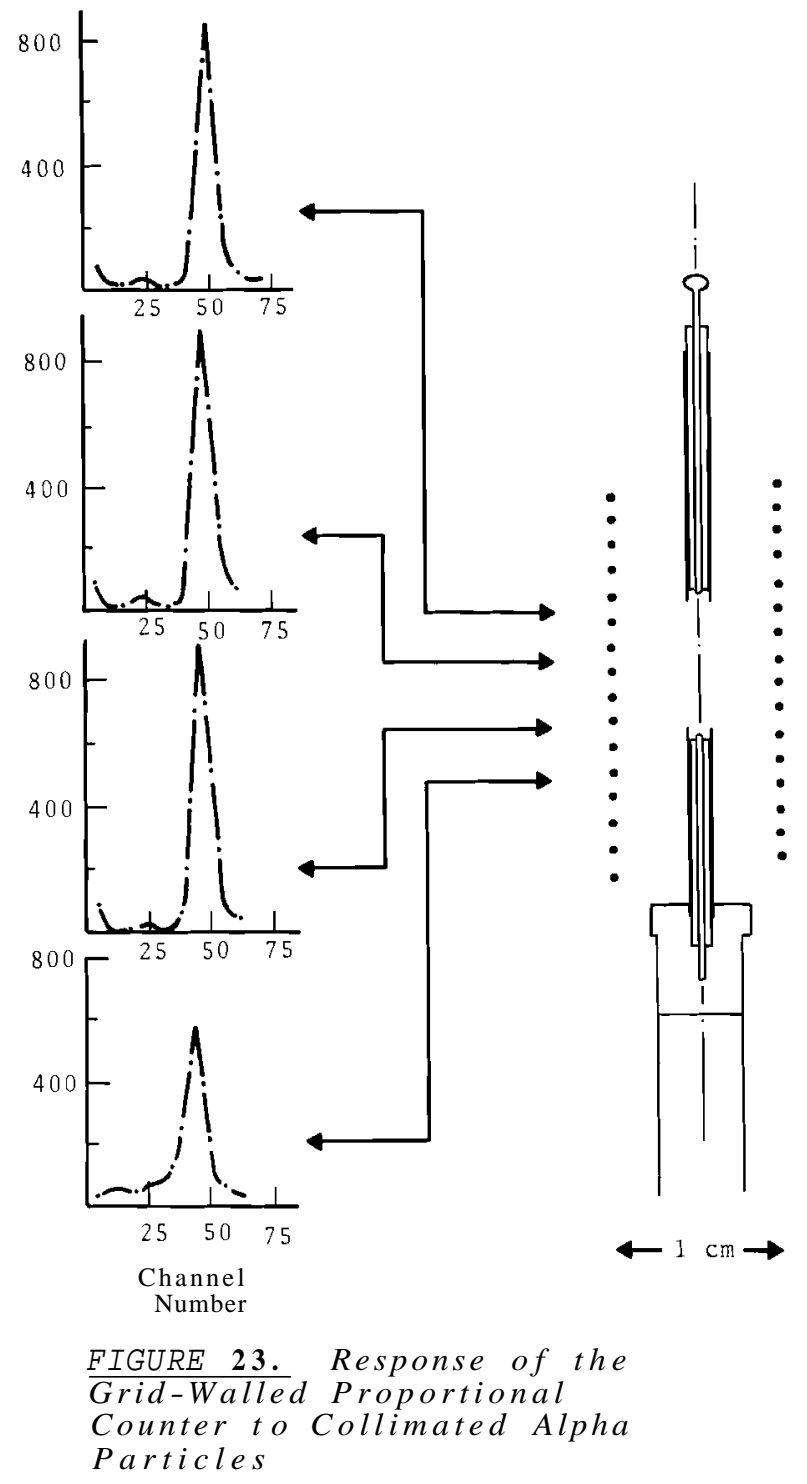

the same as its diameter, field-shaping electrodes were necessary. They are thin-walled tubes $1.6 \mathrm{~mm}$ diam. The field-shaping electrodes are insulated from the anode supports by Teflon bushings which also center the anode.

In operation, the potential on the field-shaping electrodes is adjusted to minimize distortion of the cylindrical electric field over the length of the anode. The anode is a stainless steel wire $0.076 \mathrm{~mm}$ diam. The counter is designed to operate at the center of a $122 \mathrm{~cm}$ diam spherical tank containing ${ }^{14}$ C-1abelled ethylene at a pressure of about 200 torr, which is just adequate to assure beta-ray equilibrium. At this pressure the counter will simulate a unit density site of approximately $3 \mu$.

The counter was tested for resolution and uniformity of response by directing collimated monoenergetic alpha particles across the counter normal to its axis. Figure $\mathbf{1 3}$ shows the response of the counter to these particles as they enter at different locations along the axis of the counter. The response is essentially the same at a 11 points.

\author{
SELF-ABSORPTION IN SCINTILLATION CRYSTALS-O. N. Samsky
}

\begin{abstract}
Self-absorption in scintillators implies that there is partial overlapping of their emission and absorption spectra. The intensity of light, as measured by a photomuztiplier tube, decreases as the path traveled by the light through the scintiZator increases. Optical filters enhance the effect of selfabsorption.
\end{abstract}

A study was undertaken to measure self-absorption in scintillation crystals. The amount of self-absorption depended upon the scintillator and the distance the light traveled in the scintillator before being detected by a photomultiplier tube. A well collimated beam of protons entered the scintilla- 
tor at various distances (P) from its optically coupled surface. An optical filter, placed between the scintillator and the photomultiplier tube, enhanced the effect of the self-absorption.

The experimental apparatus is shown in Figure 14. The energy control slits

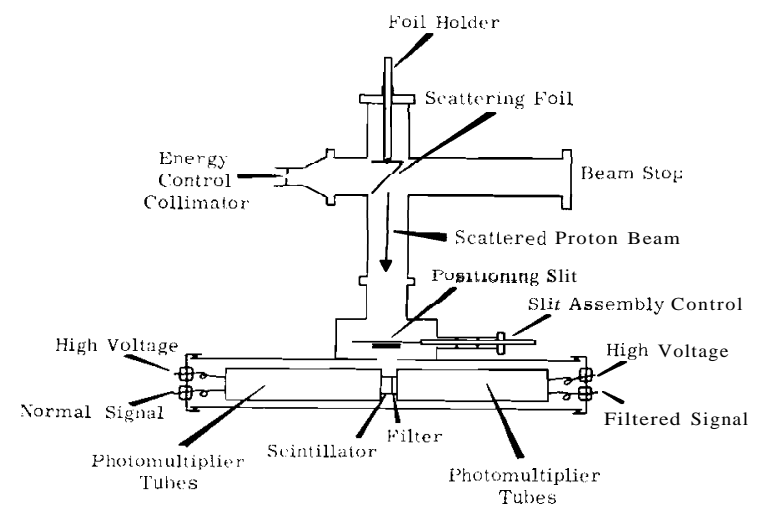

FIGURE 14. Experimental Apparatus for Determining Self-absorption in ScintilZation Crystals

and the analyzing magnet of the $2 \mathrm{MeV}$ Van de Graaff generator were used to define unique proton energies ranging from 0.3 to $1.8 \mathrm{MeV}$. A thin $(1.3 \mu)$ nickel scattering foil was used to reduce the intensity of the beam to $1 / 10^{6}$ of the original. The foil, mounted so that it could be rotated in and removed from the path of the beam, was chosen to minimize energy loss and energy spread in the scattered beam.

The energy lost in the foil in its normal position was found by rotating the foil and by using a solid state detector to measure differences in pulse height. Figure 15 defines the normal position $A$ and the rotated position $B$ of the scattering foil. With the foil in position $B$, the maximum response of the detector was used as a calibration of the machine energy
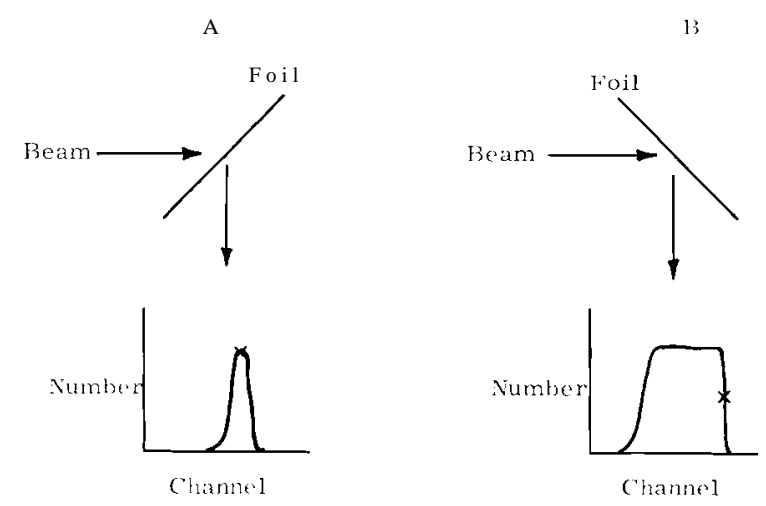

FIGURE 15. Scattering Foil Positions for Proton Energy Loss Measurements

With the foil in position W, the detector was used to measure the average energy of the proton after scattering. Figure 16 gives the relationship between the machine energy and the energy of the protons used.

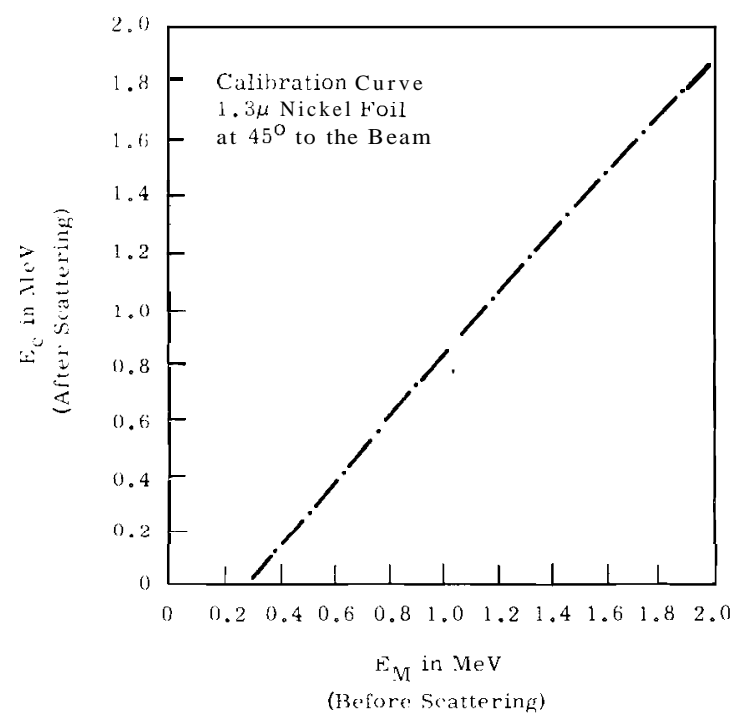

FIGURE 16. Energy Rezationship for Proton Scattering at $90^{\circ}$ Through a 1.3 w Nickel Foil

The scattered beam passed through a positioning slit. The width of the s lit was adjustable and was set at $0.13 \mathrm{~mm}$ with a feeler gage. The slit assembly, controlled by rotating a 
hexagonal nut on a 0.7874 thread per mm (20 threads/in.) bolt, gave a position reproducible to within $0.05 \mathrm{~mm}$. The beam was allowed to enter the scintillator at positions (P) ranging from 0.13 to $13.0 \mathrm{~mm}$ away from its face. The beam intensity, variable from a few to several thousand protons per second, was controlled by the energy slit separation and the intensity of the beam current.

A scintillator and a filter were optically coupled to the two Amperex 56 UVP photomultiplier tubes by silicone grease. The scintillators, anthracene and NE102, were polished discs $19 \mathrm{~mm}$ thick and $38 \mathrm{~mm}$ diam. The optical filters* have transmission curves as given in Figure 17. Figure 18 is a schematic of the electronics. The linear pulses from the photomultiplier tubes were fed into their respective preamplifiers and discriminator-amplifiers. A 11 but the smallest noise pulses caused discrim inator pulses to be fed into the coincidence unit. One of the linear pulses

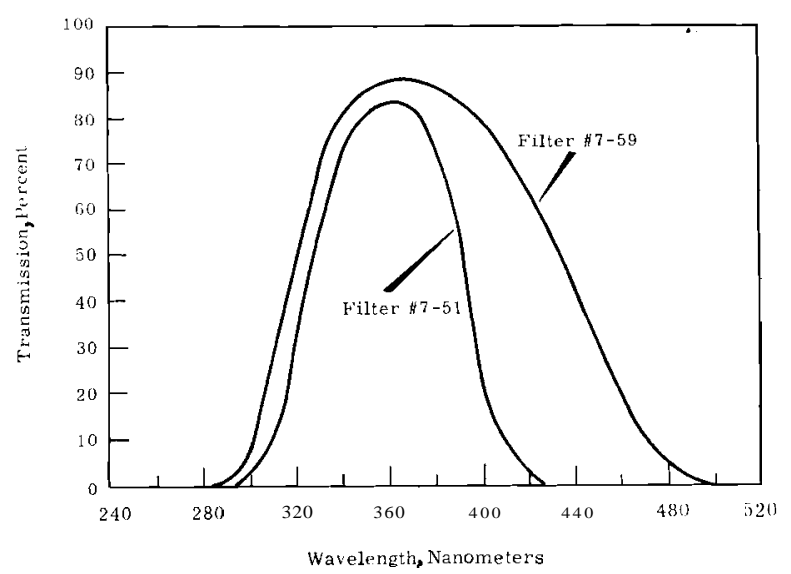

FIGURE 17. Transmission Curves for the Optical Filters

*Corning glass filters \#7-51

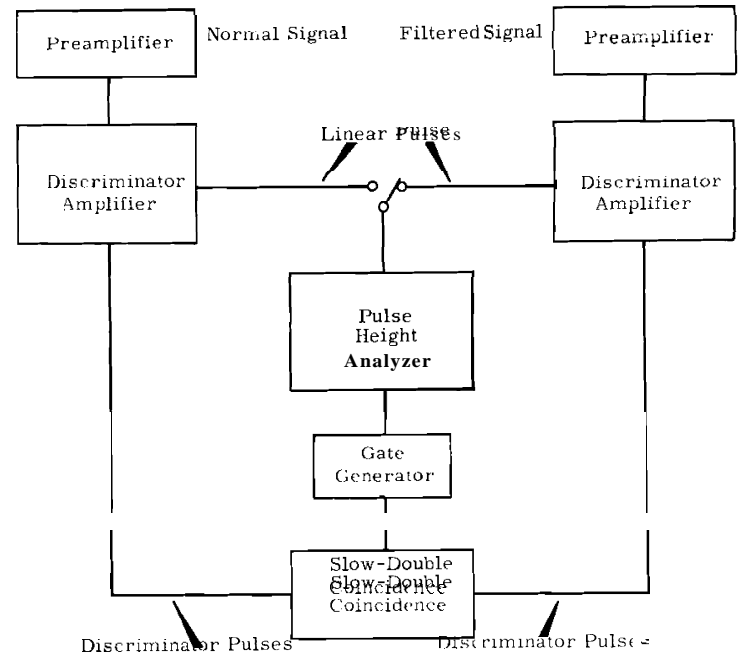

FIGURE 18. Electronic Scheme for Self-absorption in ScintizZation Crystals

was accepted for analysis by the pulse height analyzer if accompanied by a gate pulse from the coincidence unit. This coincidence gating greatly reduced the effect of photomultiplier noise.

The shift in the pulse height was measured by recording the average channel number for each position of the positioning collimator. Each measurement-a response versus position curvewas made with two scans in opposite directions so that the last points taken checked the first points for drifts in gain. Any run with a gain shift was not used. In addition, for various fixed positions, a response versus energy curve was generated by varying the accelerator energy of the Van de Graaff.

The results are shown in Figures 19 through 25. Figures 19 and 22, response versus energy curves, show that as the transmission of the filters decreases, the minimum detectable energy increases. The general shape of the curves, however, indicates that the response of the filtered photomultiplier tube can be related to the 


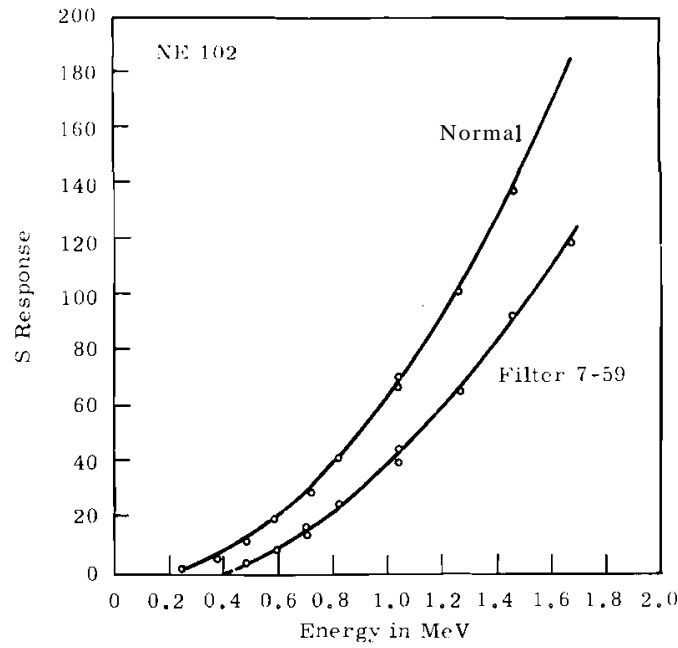

FIGURE 19. Response versus Energy Curves for NE102 for the Normal and Filtered \#7-59

Cases

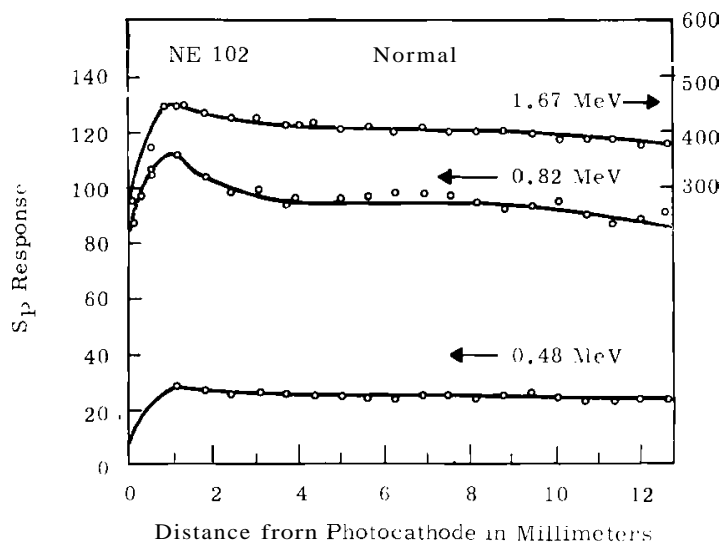

FIGURE 20. Response versus

Position Curves for the Normal

Case of NE102 at $1.67 \mathrm{MeV}$,

$0.82 \mathrm{MeV}$, and $0.48 \mathrm{MeV}$

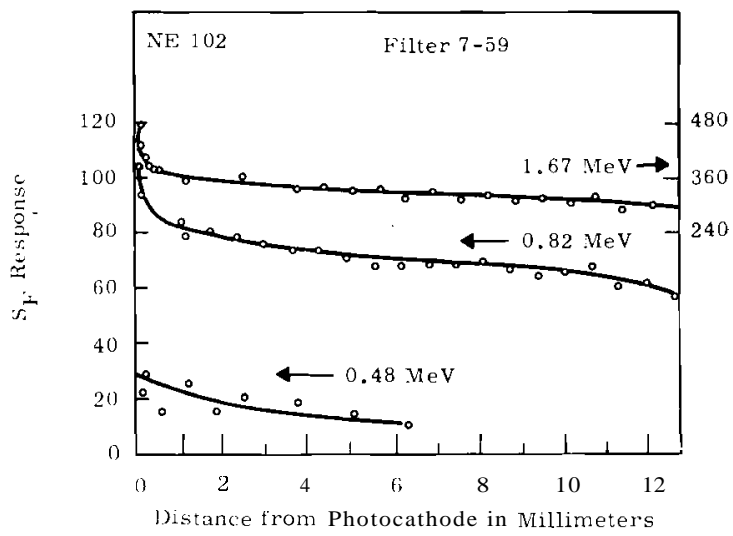

FIGURE 21. Response versus Position Curves for the Filtered \#7-59 Case of NE102 at $1.67 \mathrm{MeV}, 0.82 \mathrm{MeV}$, and $0.48 \mathrm{MeV}$

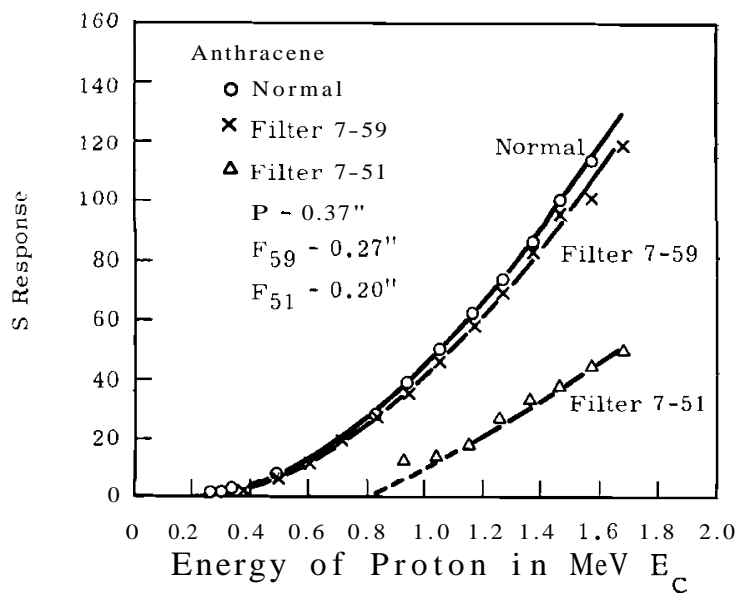

FIGURE 22. Response versus Energy Curves for Anthracene for the Normal and Filtered \#7-59 and 7-51 Cases.

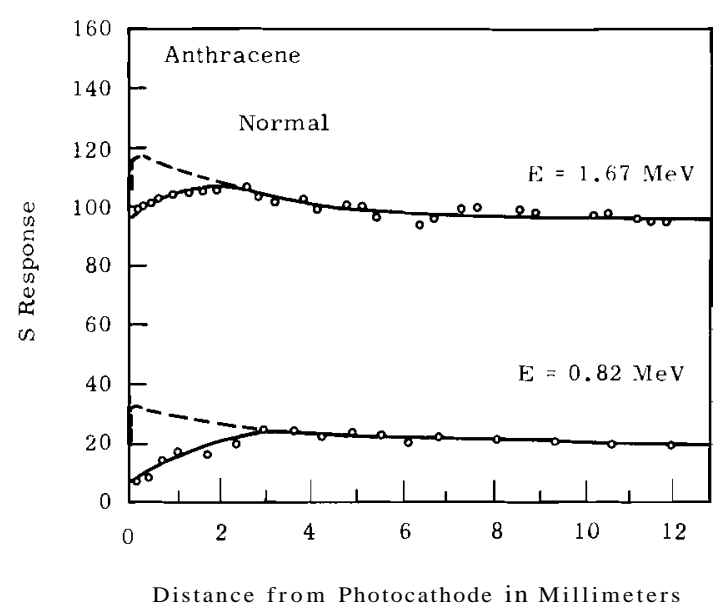

FIGURE 23. Response versus

Position Curves for the Normal

Case of Anthracene at $1.67 \mathrm{MeV}$ and $0.82 \mathrm{MeV}$

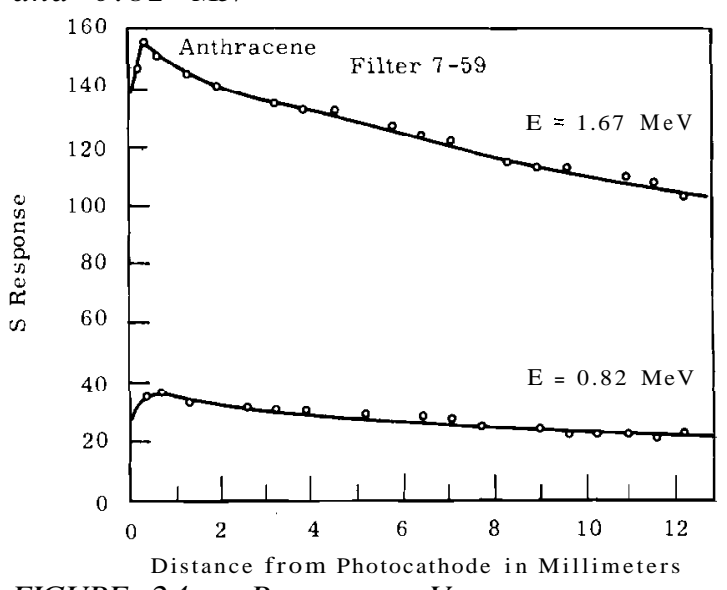

FIGURE 24. Response Versus

Position Curves for the Filter-

ed \#7-59 Case of Anthracene

at $1.67 \mathrm{MeV}$ and $0.82 \mathrm{MeV}$ 


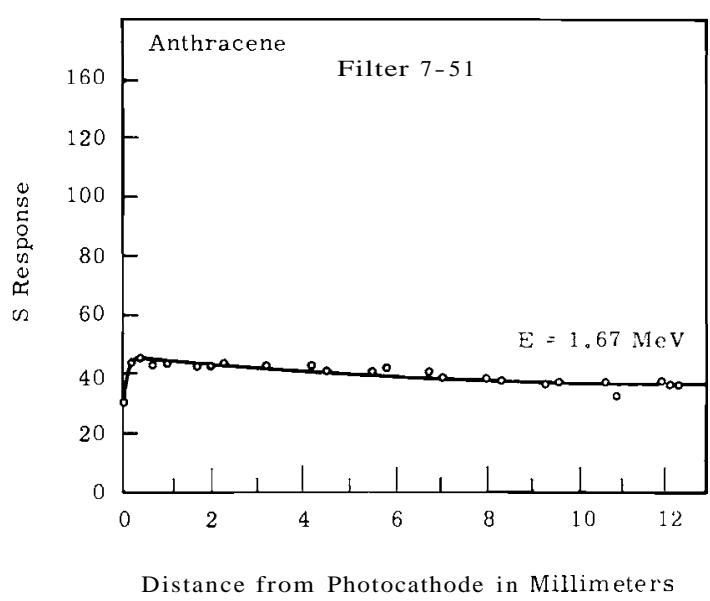

FIGURE 25. Response Versus Position Curve for the Filtered \#7-51 Case of Anthracene at $1.67 \mathrm{MeV}$

energy of the foil scattered protons. The remaining figures show self-absorption effects for anthracene and NE102, with filters \#7-59 and \#7-51 at energies of $1.67,0.82$, and 0.48 $\mathrm{MeV}$. As the position (P) increases from zero, the response reaches a maximum value. In this region, the effect of photomultiplier tube coupling predominates. As the point of scintillation approaches the photomultiplier tube scintillator interface, the effective area of coupling decreases, and hence the light reaching the photomultiplier tube also decreases. After the maximum the response curves are seen to drop off, first rapidly and then at a slower rate. This decrease in pulse height amplitude can be attributed to self-absorption in the scintillator. The effect can be explained by realizing that when ionizing radiation passes through a scintillator, it excites a primary emission spectrum that in most cases partially overlaps the absorption spectrum for that scintillator. Thus, for a given wave length the intensity of the initial light will fall off expotentially with distance. The absorption coefficient is determined from the absorption spectrum of the scintillator. The final transmitted light is a summation of all the exponential terms. This relation has been put in approximate analytical form by Kaiser and deVilliers. (4) Their measurements, however, were made using scintillators thicker than $8 \mathrm{~mm}$ and using $244 \mathrm{Cm}$ alphas as a source. No attempt has been made here to form an analytical expression of the self-absorption. The effect of the filters was to reduce the amount of light that reached the photomultiplier tube that was nonabsorbable in the scintillator. For NE102, filter \#7-51 was not used since the intensity of light that it passed was not sufficient for analysis.

The self-absorption in a scinti11ator can be measured in a single crystal using protons. The absorption effect is a function of path length to the photomultiplier tube and can be enhanced by appropriate use of optical filters. The effect seems to be energy independent.

PULSED X-RAY MACHINE- L. A. Braby

Evaluation of the $600 \mathrm{kv}, 12 \mathrm{~kJ}$ pulsed X-ray machine* was continued.

*Field Emission Corporation,

McMinnvizie, Oregon.
A problem in early tests of the pulser was the inconsistency of current and voltage measurements using the load resistors provided. The value of these 
resistors seemed to change both with voltage and time. We learned that they were made up of high power, low-value carbon film resistors connected in series and that others had experienced similar deterioration under pulse use. It was suggested that resistive elements of copper sulphate in aqueous solution be used between copper electrodes. Such resistors were known to work reliably with very short pulses, but they hau not been used for pulses longer than 1 usec. They have a relatively high temperature coefficient of resistance; therefore, the volume and resistivity of the solution must be adjusted to provide sufficient thermal capacity so that the temperature change caused by power dissipation will be less than a fraction of a degree. A Lucite cylinder 9 om diam and $1 \mathrm{~m}$ long, containing approximately $0.05 \mathrm{M} \mathrm{CuSO}_{4}$, makes a $300 \Omega$ resistor which increased in temperature about $1 / 3{ }^{\circ} \mathrm{C}$ with a 12 $k J$ pulse. Tests showed no problem with 10 psec pulses, and successive current measurements agreed within the accuracy of the oscilloscope readout. From these tests, it appears that the power supply is performing satisfactorily; however, no lifetime or endurance tests have been made. Experiments with this device would involve the operation of photomultiplier tubes and other circuitry placed near the pulser. Since the spark gaps are unshielded, the device radiates a large amount of RF noise. To keep this noise from the data signals, a photomultiplier and preamplifier were shielded with a $1 \mathrm{~cm}$ thick aluminum can and connected to display equipment by a single cable thus avoiding any possibility of ground loops. Tests indicated that these precautions reduced the noise on the output signal to a reasonable level for preliminary experiments.

LOCAL ENERGY DENSITY AND HIT THEORY-W. C. Roesch

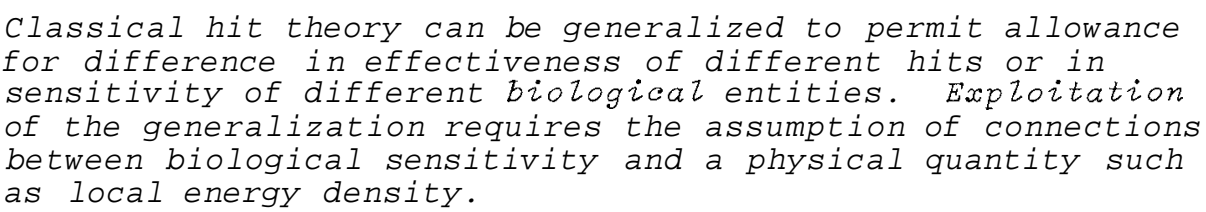

Hit "theory" is a hypothesis to explain the observed forms of dose-effect curves by relating them to elementary quantized radiation absorption events (hits) distributed according to Poisson statistics. For example, if a single hit within a biological entity is sufficient to kill it, then the fraction surviving a dose $D$ is where $m$ is the average number of hits expected in a biological entity and $\lambda=(\mathrm{m} / \mathrm{D})=$ constant is the average number expected per unit dose. Zimmer recently reviewed the subject of hit theory ${ }^{(6)}$. Among the criticisms he noted that had been made of the theory were that the mathematical analysis had not been developed to allow for 
the difference in efficiency of different interactions or for the difference in radiosensitivity of diferent biological entities. This paper supplies the necessary mathematics. In the first part of the paper the necessary formal changes are made to classical hit theory. In the second part a connection with local energy density is assumed in order to exploit the developments in the first part; some other way of associating biological and physical events on the microscopic scale might do equally well or better.

H it Theory

It is assumed that each hit produces its effects on the biological entity independently of every other hit. Then there must be a probability of survival, $\mathrm{S}_{1}$, following a single hit. This $\mathrm{S}_{1}$ is determined by the range of effectiveness of individual hits and by the differences in radiosensitivity of different entities in the irradiated population. Because of the assumed independence of the effects of different hits, the probability of surviving $n$ hits is $s^{n}$. The probability of $\mathrm{n}$ hits, when the mean number expected is $\mathrm{m}$, is

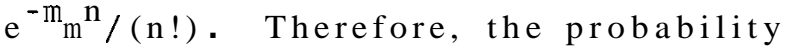
of surviving such a dose is

$$
S=\sum_{n=0}^{\infty} e^{-m} m^{n} s_{1}^{n} /(n !)=e^{-m\left(1-s_{1}\right)} .
$$

From local-energy-density theory ( 7$)$ $D=m\langle\Delta Z\rangle$. This can be introduced in Equation (2) as a physical relationship without any implication regarding a connection of effectiveness or sensitivity with local energy density.

$$
S=e^{-k D}
$$

with

$$
k=\frac{\left(1-S_{1}\right)}{\langle\Delta Z\rangle}
$$

At this point any of the usual more elaborate forms of hit theory can be built up from Equations (2) or (3).

For example, if each of $N$ entities in a system must be destroyed to kill the system, then the probability of survival of a system is $1-\left(1-e^{-k D}\right)^{N}$ with $e^{-k D}$. from Equation (3). If destruction of any one of the $\mathrm{N}$ will kill the system, then the probability of survival is $\mathrm{e}^{-\mathrm{kDN}}$, e tc.

The Relative Biological Effectiveness (RBE) of a radiation, denoted by h, relative to $x$ rays, denoted by $x$, is

$$
\mathrm{RBE}=\mathrm{k}_{\mathrm{h}} / \mathrm{k}_{\mathrm{x}}
$$

whether the survival curve is a simple exponential such as Equation (3a) or one of the more complicated functions just considered.

If a specific radiobiological phenomenon is to be described by the above equations, a single type of survival curve [Equation (3), one of the N-entity equations, or others] must fit the data for all types of radiation by changing only the value of $k$. There is no difficulty in seeing how this is possible if the curves are all simple exponent i a $1 \sim$. Another type of family of survival curves often seen is that of exponential curves for high LET radiation with more complex curves, similar to multi-hit curves, for low LET. How this might arise is shown in Figure 26. Here $1-\left(k-e^{-k D}\right)^{2}$ is shown for $k^{\prime} s$ in the ratio $1: 4: 8$. All the curves are the same shape, but, one the scale chosen, the higher RBE curves look exponential. 


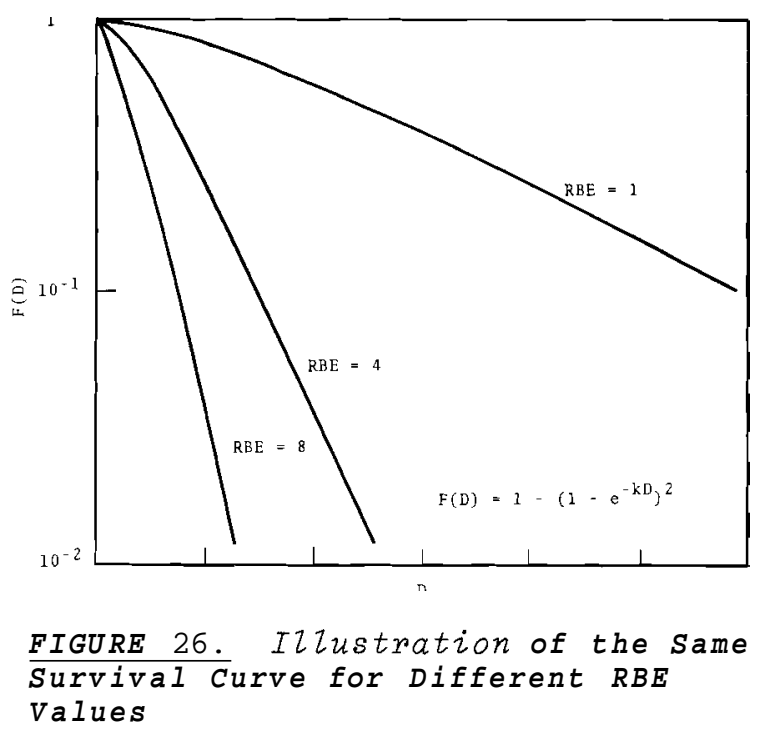

A remaining question, then, is, if Equation (3b) will give the necessary variation of $\mathrm{k}$ with LET. To answer this question requires additional assumptions about $S_{1}$. It must be assumed to vary with some quantity that describes the radiation. We will assume for the moment that the local energy density introduced by Rossi, Biavati, and Gross (8) is the necessary quantity.

\section{Local Energy Density}

Assume that in a hit in which there is an increment $\Delta Z$ to the local energy density there is a probability of survival s $(\Delta Z)$. Then

$$
S_{1}=\int_{0}^{\infty} s(\Delta Z) P(\Delta Z) d \Delta Z .
$$

One expects a priori that $s(\Delta Z)$ is nearly unity for small $\Delta Z$ and that it decreases monotonically with increasing $\Delta Z$.

$$
\begin{aligned}
& \text { Taking } \\
& \qquad S(\Delta Z)=e^{-\lambda \Delta Z}
\end{aligned}
$$

corresponds to the classical single-hit theory. Equations (5) and (3b) give

$$
k=\left(1-\left\langle e^{-\lambda \Delta z}\right\rangle\right) /\langle\Delta z\rangle
$$

where the notation is that of reference ${ }^{(7)}$. If $X A Z \ll 1$ over most of the $\Delta Z$ distribution, the exponential in Equation (7) can be replaced by the first two terms of its power series expansion. This gives $\mathrm{k}=\lambda=$ constant. The survival curves are exponential, and the RBE's are the same for all radiations. Keeping another term in the series shows that the RBE of high LET radiations is slightly less than that for low LET; this is what is observed in single-hit phenomena. If $\lambda \Delta Z>>1$, the exponential in Equation (7) can be neglected and $\mathrm{k}=\langle\Delta \mathrm{Z}\rangle^{-1}$. This gives very low RBE's for high LET radiations and is seldom of interest in radiobiology.

Calculations with other possible functions for $s(\Delta Z)$ indicate that much of the shape of RBE curves can be understood from the behavior of $s(\Delta Z)$ for small $\Delta Z$. If, for example, with $\mathrm{n}>1$

$$
s(\Delta Z) \simeq 1-\Delta Z^{n}
$$

for small $\Delta \mathrm{Z}$, Equation (3b) gives

$$
\mathrm{k} \simeq\left\langle\Delta \mathrm{z}^{\mathrm{n}}\right\rangle /\langle\Delta \mathrm{z}\rangle
$$

Then k will be small for low LET, rise to a maximum, and decrease as $\langle\Delta\rangle\rangle^{-1}$ for high LET. This is how many RBE curves behave. 
The change in neutron yiezd of two Pu-Be neutron sources with time was determined to be $1.0 \pm 0.3 \%$ per year. The accuracy of result is dependent on the stability of the $B F_{3}$ tube sen-
sitivity.

The flux from Pu-Be neutron sources changes as a function of time. This is caused primarily by the build-up of 241 Am from the original ${ }^{241} \mathrm{Pu}$ in the source. Since these sources are used as flux standards in neutron experiments, it is importnat to know how the output changes with time.

A previous attempt ${ }^{(9)}$ to measure this change by comparing the unknown source with a known ${ }^{238} \mathrm{Pu}-\mathrm{Be}$ source was unsuccessful. The change in neutron emission rate was calculated using the known isotopic composition and the known number of neutrons per alpha. The source was made from a powdered mixture of $\mathrm{PuO}_{2}$ and beryllium instead of the more stable PuBe ${ }_{13}$ complex, and it failed to yield reproducible results. Previous attempts to measure secular changes using a $\mathrm{BF}_{3}$ tube as a standard were hampered by radiation damage effects. (10) More recently, however, tubes have been obtained that are considerably less sensitive to this damage.*

The sources were positioned inside a precision long counter(11) and the count rate for each source was measured periodically over a 2 yr period. The data were fitted with a straight line and the percent change per year was calculated. For our two standard sources $\left(M-246^{\prime}, M-710^{\prime}\right)$ the neutron flux increases by $1.0 \pm 0.3 \% /$ year.

The uncertainty in this value was determined by estimating the magnitude of the known sources of error. These errors include the standard deviation due to counting statistics, a small error in the technique used to set the gain of the $\mathrm{BF}_{3}$ tube counting system, and an error caused by density changes in the polyethylene in the long counter brought about by variations in room temperature.

* Twentieth Century Electronics, New Addington, Croyden, Surrey, England

The labor of determining the Zocal-energy-density distribution can often be avoided by reducing the desired quantity to an expression involving only moments of the single-event distribution or by making use of the Gaussian approximation when the expected number of events is large.

Rossi, Biavati, and Gross (8) intro- and biological effects of ionizing radiduced the quantity "local energy density", ation at the microscopic level. The $Z$, to assist in relating the physical local energy density is the energy ab- 
sorbed in a small spherical volume of matter divided by the mass of the matter in that volume. For spheres small enough to be of radiobiological interest, a few microns and less in diameter, a given absorbed dose will produce different values of the local energy density in different spheres because the particle nature of radiation results in concentration of absorbed energy along particle tracks rather than in uniform distributions throughout the irradiated material. Determining the local-energy-density distribution, $P(Z)$, is the first task. One or more events may contribute to $Z$. Determining $P(\Delta Z)$ the distribution of increments $\Delta Z$ to $Z$ in single events, is easier than determining $P(Z)$. The purpose of this paper is to demonstrate mathematical relations between $P(\Delta Z)$ and $P(Z)$ that frequently permit avo+-ding the determination of $P(Z)$.

Rossi(12) has given a formula for calculating $P(Z)$ from $P(\Delta Z)$ :

$$
P(z)=\sum_{n=0}^{\infty} e^{-m_{m} n}(n !)^{-1} P_{n}(z)
$$

where

$$
P_{n}(Z)=\int_{0}^{z} P\left(\Delta Z^{\prime}\right) P_{n-1}\left(z-\Delta Z^{\prime}\right) d \Delta Z^{\prime}
$$

and

$$
P_{0}(Z)=\delta(Z),
$$

the Dirac delta function. $P_{n}(Z)$ is the probability of a local energy density $\mathrm{Z}$ in $n$ events; $\mathrm{e}^{-\mathrm{m}_{\mathrm{m}} \mathrm{n}}(\mathrm{n} !)^{-1}$ is the Poisson probability of $n$ events when the average number is $\mathrm{m}$. Equations(1) through (3) give $P(Z)$ when $P(\Delta Z)$ is known, but usually much computational labor is involved.

Let us use the notation

$$
\langle f(z)\rangle=\int_{0}^{\infty} f(z) P(z) d z .
$$

We will calculate $\left\langle e^{-\lambda z}\right\rangle$. This is the Laplace transform of $P(Z)$; if the sign of $\lambda$ were changed, it would be what is known in probability theory as the moment-generating function. Putting $f(Z)=e^{-\lambda Z}$ in Equation (4), substituting $P(Z)$ from Equation ( 1 ), and using the Laplace transform convolution theorem $n$ times in the n $\frac{\text { th }}{\text { term gives }}$

$$
\left\langle e^{-\lambda z}\right\rangle=e^{-m\left[1-\left\langle e^{-\lambda \Delta z}\right\rangle\right]}
$$

where the brackets in $\left\langle e^{-\lambda \Delta z}\right\rangle$ denote the same operation as in equation (4), but the presence of the $A$ indicates that the integration is over the distribution $P(\triangle Z)$ rather than $P(Z)$.

It is readily shown that

$$
\left\langle z^{n} e^{-z}\right\rangle=\frac{(-1)^{n} d^{n}\left\langle e^{-\lambda z}\right\rangle}{d \lambda^{n}}
$$

From this and Equation (5)

$$
\begin{aligned}
\left\langle e^{-\lambda z}\right\rangle= & e^{-m}\left[1-\left\langle e^{-\lambda \Delta z}\right\rangle\right] \\
\left\langle z e^{-\lambda z}\right\rangle= & m\left\langle\Delta z e^{-\lambda \Delta z}\right\rangle e^{-m[1} \\
& \left.-\left\langle e^{-\lambda \Delta z}\right\rangle\right] \\
\left\langle z^{2} e^{-\lambda z}\right\rangle= & {\left[m^{2}\left\langle\Delta z e^{-\lambda \Delta z}\right\rangle^{2}\right.} \\
& \left.+m\left\langle\Delta z^{2} e^{-\lambda \Delta z}\right\rangle\right] e^{-m[1} \\
& \left.-\left\langle e^{-\lambda \Delta z}\right\rangle\right]
\end{aligned}
$$




$$
\begin{aligned}
\left\langle z^{3} e^{-\lambda z}\right\rangle= & {\left[m^{3}\left\langle\Delta z e^{-\lambda \Delta z}\right\rangle^{3}\right.} \\
& +3 m^{2}\left\langle\Delta z e^{-\lambda \Delta z}\right\rangle\left\langle\Delta z^{2} e^{-\lambda \Delta z}\right\rangle \\
& \left.+m\left\langle\Delta z^{3} e^{-\lambda \Delta z}\right\rangle\right] e^{-m}[1 \\
& \left.-\left\langle e^{-\lambda \Delta z}\right\rangle\right]
\end{aligned}
$$

etc., and, by letting $A=0$,

$$
\begin{aligned}
\left\langle z^{0}\right\rangle= & 1 \\
\langle z\rangle= & m(A z) \\
\left\langle z^{2}\right\rangle= & m^{2}\langle\Delta z\rangle^{2}+m\left\langle\Delta z^{2}\right\rangle \\
\left\langle z^{3}\right\rangle= & m^{3}\langle\Delta z\rangle^{3}+3 m^{2}\langle\Delta z\rangle\left\langle\Delta z^{2}\right\rangle \\
& +m\left\langle\Delta z^{3}\right\rangle
\end{aligned}
$$

etc.

The mean value of $Z,(Z)$, equals the absorbed dose; ${ }^{(9)}$ therefore, from Equation ( $8 \mathrm{~b}$ )

$$
D=m\langle\Delta z\rangle \text {. }
$$

Equation (8a) says that the $P(Z)$ distribution is normalized to unity. It is so normalized because it includes the term $\mathrm{e}^{\mathrm{m}_{\delta(}(z)}$ for the probability of no energy deposition event taking place (the probability of an event taking place but with no energy deposition is $P(A Z=0) d \triangle Z)$. Determinations of $P(Z)$ do not include this term but give instead $\mathrm{P}^{*}(\mathrm{Z})=\mathrm{P}(\mathrm{Z})-\mathrm{e}^{-\mathrm{m}} \delta(\mathrm{z}) \cdot \mathrm{P}^{*}(\mathrm{z})$ is normalized to $1-\mathrm{e}^{-\mathrm{m}}$ rather than unity. Using * to denote the operation of Equation (4) but with $\mathrm{P}^{*}(\mathrm{z}) /\left(1-\mathrm{e}^{-\mathrm{m}}\right)$ gives

$$
\langle z\rangle^{*}=\frac{m\langle\Delta z\rangle}{1-e^{-m}}
$$

and

$$
\begin{aligned}
\sigma^{2} & =\left\langle z^{2}\right\rangle^{*}-\langle z\rangle^{*} 2 \\
& =\frac{m\left\langle\Delta z^{2}\right\rangle}{1-e^{-m}}-\frac{m^{2}\langle\Delta z\rangle^{2} e^{-m}}{\left(1-e^{-m}\right)^{2}} .
\end{aligned}
$$

For m much less than one, $\langle z\rangle^{*}$ approaches $\langle\Delta z\rangle$ and $\sigma^{2}$ approaches $\left\langle\Delta z^{2}\right\rangle-\langle\Delta z\rangle^{2}$, i.e., $\mathrm{P}^{*}(Z) /\left(1-\mathrm{e}^{-\mathrm{m}}\right)$ approaches $\mathrm{P}(\Delta Z)$ or $\mathrm{P}^{*}(Z)$ essentially equals $m P(\Delta Z)$. For $m$ much larger than one, $\langle z\rangle^{*}$ approaches $m\langle\Delta z\rangle$ and $\sigma^{2}$ approaches $\mathrm{m}\left\langle\Delta z^{2}\right\rangle$; higher odd central moments approach zero. This indicates that $P^{*}(Z)$ [or $P(Z)$ ] approaches

a Gaussian distribution

$$
\begin{aligned}
P^{*}(z)= & {\left[2 \pi D\left\langle\Delta z^{2}\right\rangle /\langle\Delta z\rangle\right]-1 / 2 } \\
& \exp \left[(z-D)^{2}\langle\Delta z\rangle / 2 D\left\langle\Delta z^{2}\right\rangle\right] .
\end{aligned}
$$

Applications seldom require $P(Z)$ it self; they often require some expression of the form of Equation (4). For example, Biavati(13) used $f(=)$ equal to one for $Z \leq Z_{n}$ and equal to zero for $Z>Z_{\circ}$. This can be evaluated

$$
\frac{1}{2}\left\{1+\operatorname{erf}\left[\frac{z_{0}-D}{\left(\frac{2 D<\left\langle z^{2}\right\rangle}{\langle\Delta z\rangle}\right)^{1 / 2}}\right]\right\}
$$

for large m.

More generally, $f(Z)$ can be approximated by a power series. Or, an expression of the form

$$
f(z)=e^{-A Z}\left(a_{0}+a_{1} z+a_{2} z^{2}+\ldots\right)
$$

may be a better approximation. In this case

$$
\langle f(z)\rangle=a_{0}\left\langle e^{-\lambda z}\right\rangle+a_{1}\left\langle z e^{-\lambda z}\right\rangle+\ldots
$$


and the terms on the right can be evaluated by Equations (8).

Sometimes one wants just the event frequency

$$
1-e^{-m}=1-e^{-D} /\langle\Delta z\rangle
$$

from Equation (9).

\section{CALORIMETRIC MEASUREMENT OF ${ }^{210} \mathrm{Po}^{*}$-D. M. Fleming}

A calorimeter of the twin type(14) was used for cylindrical samples as large as $24 \mathrm{~cm}$ long and $5 \mathrm{~cm}$ diam. Thermal insulation was obtained by a - $1.3 \mathrm{~cm}$ layer of styrofoam. The calorimeter relied on the high thermal conductivity of a $3.2 \mathrm{~mm}$ thick copper jacket to provide the same temperature environment for each side. Temperature rise of the system was 4.2 ${ }^{\mathrm{O}} \mathrm{C} / \mathrm{W}$. A 12-junction, iron-constantan thermopile was used to detect any temperature difference between cells. Thermopile output was fed to an amplifier which in turn supplied a signal to a proportional control system.

The controller provided the calibration side of the calorimeter with the electrical power necessary to keep the two sides at the same temperature. At equilibrium the heat output of the sample is equal to the power supplied to the calibration heater. A clock- controlled readout system recorded the voltage on the heater at predetermined intervals. The system was therefore completely automated.

Maximum measurable source power is $10 \mathrm{~W}$ and the minimum detectable power level is about $10 \mathrm{~mW}$. Accuracy required for the measurement was $1 \%$ which was easily obtained. Sixteen irradiated bismuth elements were measured. The first element to be measured was remeasured at the end of the experiment to obtain the decay rate of the heat output. The measured value differed from the accepted half-life of ${ }^{210}$ Po by less than the estimated experimental error; this supports the assumption that the heat contribution from radioactive contaminants was negligible.

*This work was not funded by the Division of Biology and Medicine.

APPARATUS FOR LOCAL-ENERGY-DENSITY MEASUREMENTS-W. A. Glass

A proportional counter is described capable of measuring the distribution of energies deposited in microscopic spherical sites (of the order of $\left.5 x 20^{-12} \mathrm{~g}\right)$ by ionizing radiation. Distributions of the dose as a function of event size for monoenergetic neutrons are compared with similar results obtained at the Radiological Research Laboratory, Columbia University.

Investigation of the energy deposited by ionizing radiation in microscopic absorption sites (sites of the order of
$5 \times 10^{-12} \mathrm{~g}$ ) is made possible by measuring the energy deposited in cavities filled with a gas having the same atomic 
composition as the wall material. Theories advanced by U. Fano ${ }^{(15)}$, G. Failla ${ }^{(16)}$, and others show that when such a cavity is surrounded by sufficient material to establish charged particle equilibrium, the cavity experiences the same energy absorption per unit mass as the surrounding wall. Rossi, et a1. $(8,17,18)$ have shown that except for a possible effect due to electron scattering, the energy spectrum of ionizing events in such a cavity is the same as the spectrum in microscopic sites of higher density material. The dimensional scaling factor is the ratio of the density of the gas to the density of the material being simulated.

Techniques for producing proportional counters in spherical cavities have been developed at the Radiological Research laboratory at Columbia University Our Laboratory recently obtained one of these spherical proportional counters with tissue equivalent walls.* The following is a description of the equipment necessary for making local-energydensity measurements and of the general performance characteristics of the counter.

The proportional counter is a spherical cavity $10 \mathrm{~cm}$ diam surrounded by about $6 \mathrm{~mm}$ of tissue equivalent plastic. A cylindrical electric field about the anode, necessary to produce uniform gas multiplication for ionizing events independent of their location within the cavity, is maintained by a tenuous spiral

* The counter was made available to us through the kindness of H. H. Rossi and his co-workers at Columbia University. It was fabricated by Mr. Rudolph Gand and Mr. Robert Lee. grid held at a potential 0.2 that of the anode. This voltage is sufficient to collect electrons from ionizing events within the spherical cavity. The collected electrons pass through the transparent grid and into the cylindrical electric field between the grid and anode where the gas multiplication process takes place to produce a signal proportional to the initial ionization. Figure 27 shows construction details. Evidence that electrons are collected from all regions of the spherical volume and are subject to uniform multiplication is given in Figure 28. This figure displays the pulse height distribution resulting from a monoenergetic alphá particle source mounted flush with the counter wall. It is assumed that the alpha particles lose a sufficiently small amount of energy in traversing the cavity that their

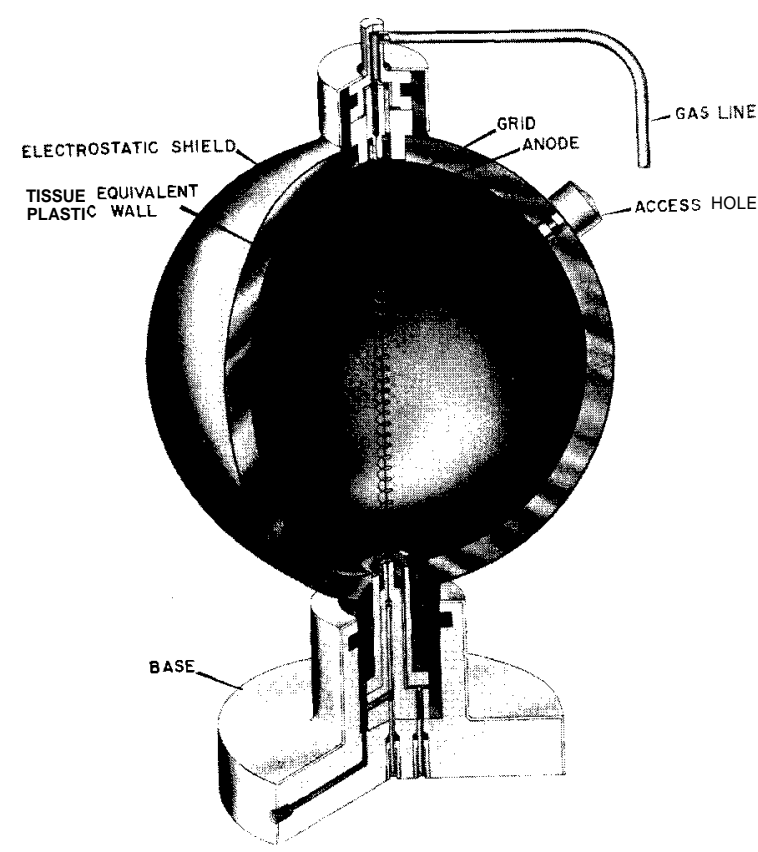

FIGURE 27. The Spherical Proportion- 


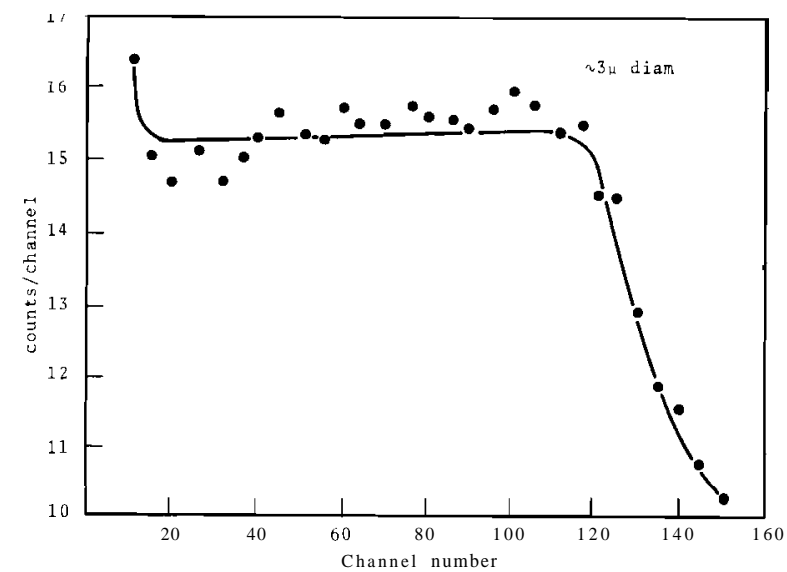

FIGURE 28. Event Spectrum Resulting from a Monoenergetic Alpha-Particle Source Mounted Flush with Counter Wall

stopping power is constant. Hence, the resulting energy loss distribution distribution of chords drawn, with equal probability in a 11 directions, from a point on the wall of the sphere. That is, all energy losses should be equally probable from zero to a maximum value which represents a traversal along the diameter; larger events obviously have zero probability.

I t was necessary to provide additional electrostatic shielding of the counter against noise pick-up. An adequate conductor was produced by vacuum deposition of about $10 \mu$ of silver onto the outside wall of the counter. This shield was then electrically connected to the counter base and the common ground.

Signals from proportional counters typically display a rather slow and variable rise time primarily due to the finite drift velocity of electrons in the gas. This is particularly true for this counter since the radial extent of ionizing events can be as great as $5 \mathrm{~cm}$. Signals of this type are best amplified with a charge-sensitive preamplifier followed by an RC amplifier with relatively long differentiation and integration time constants. Figure 29 shows the effect of amplifier time constants on resolution. The resolution is taken as the fractional fullwidth-at-half-maximum for events produced by collimated alpha particles directed across a diameter of the counter. These results indicate little or no improvement in resolution by increasing the time constants beyond $3.2 \mu \mathrm{sec}$.

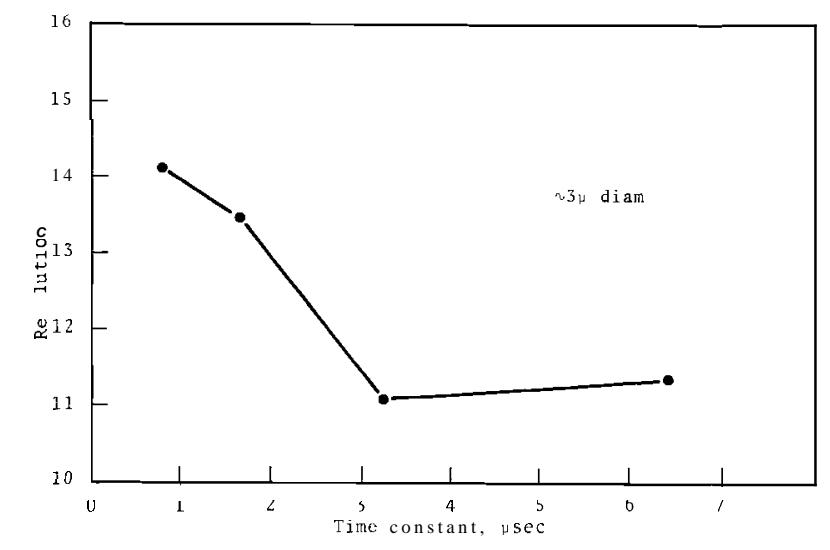

FIGURE 29. Resolution of the Spherical Proportional Counter as a Function of Amplifier Time Constants

Figure 30 shows the wide range of gas gain over which this counter will function satisfactorily. Stable operation at very high gas gain is important when measuring ionizing events produced by high energy electrons. These events may contain only a few

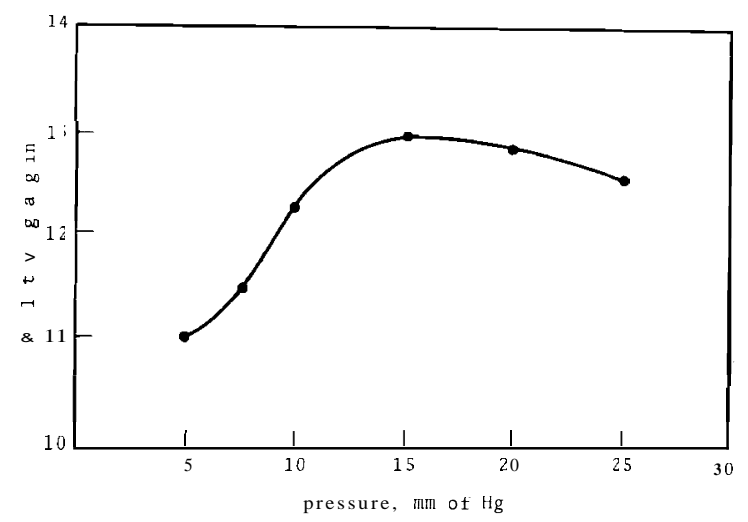

FIGURE 30. Gas Gain of the Spherical Proportional Counter as a Function of Gas Pressure 
initial ion pairs, and gas gains of $10^{3}$ or more are necessary to raise the signal above the inherent electronic noise.

Simulation of spherical absorption sites of the order of $1 \mu$ diam in tissue requires operation of the counter at gas pressures of a few torr.

The gas gain of proportional counters is a very sensitive function of pressure, particularly at pressures below 20 torr, as shown in Figure 31. Thus, for ultimate resolution, the gas flow system used with the spherica 1 proportional counter must maintain a constant pressure for the period of

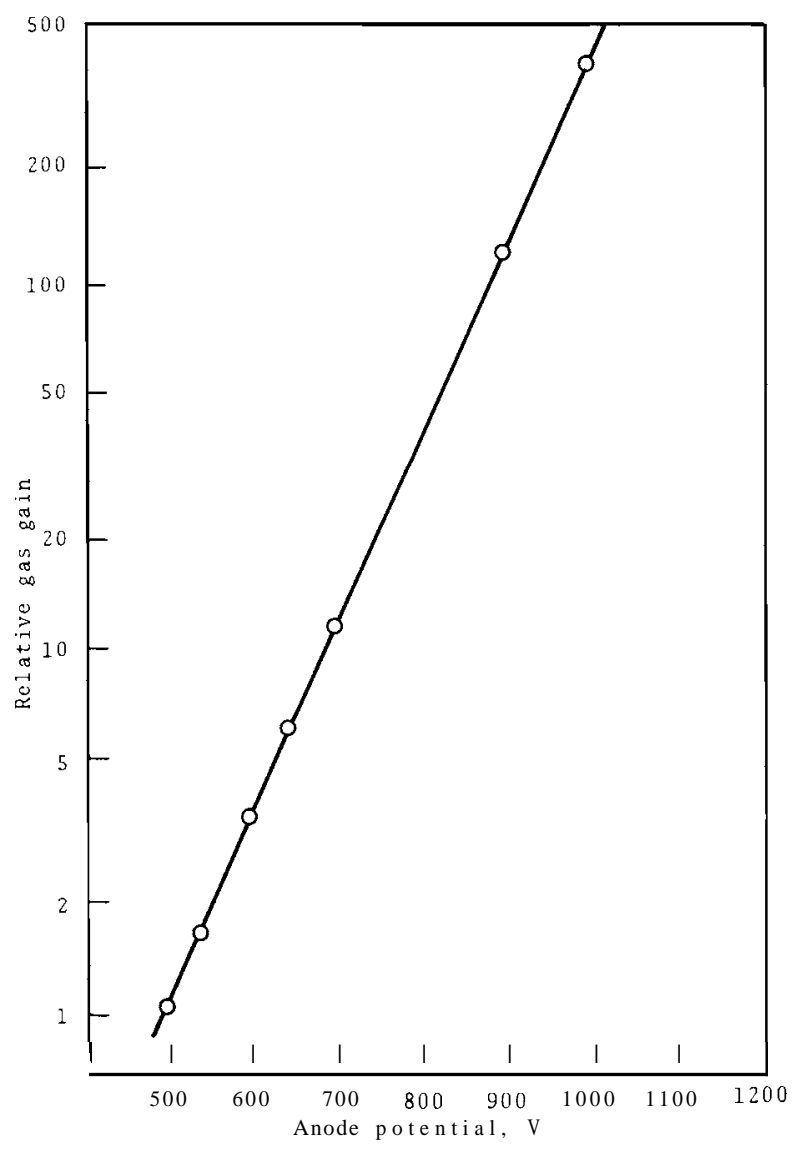

FIGURE 32. Gas Gain of the Spherical Proportional Counter as a Function of Anode Potential time required to accumulate a spectrum. Our gas handling system, simi1 ar to that reported by M. H. Biavati e t al. (19) has shown some difficulty with long term stability of gas pressure. Work has begun on an electronically controlled feedback device to regulate gas pressure with an error signal derived from gain shifts in another proportional counter operating from the same pressure system.

A comparison of our results with those obtained by the group at Columbia University, who pioneered this type of measurement, seems appropriate. A good result to compare is the dose distribution in event size for monoenergetic neutrons. Event size has been defined as the energy (measured in KeV) deposited in a spherical site divided by the equivalent diameter of that site in unit density material.

Figure 32 compares the normalized dose distributions obtained for monoenergetic neutrons in our laboratory with those reported by M. H. Biavati et a1. (20) The general features of these

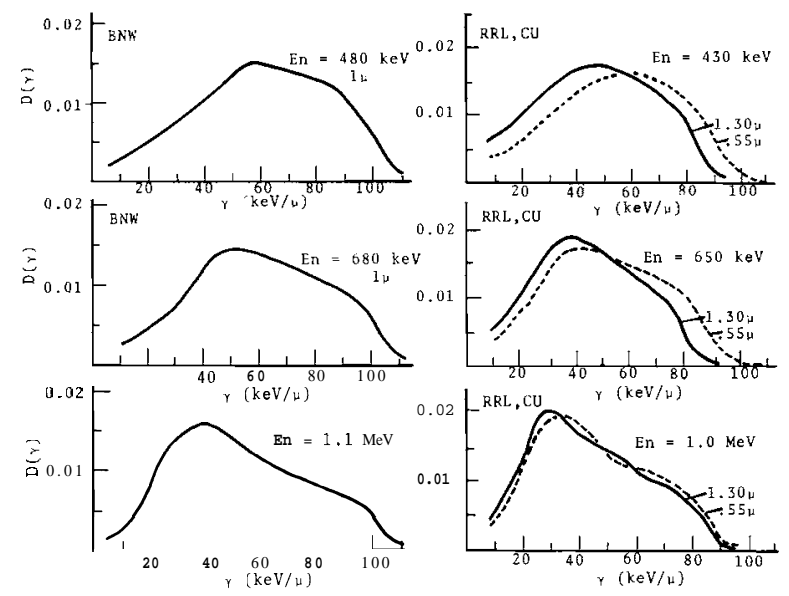

FIGURE 32. Comparison of the Dose Distribution in $Y$ of Monoenergetic Neutrons (Distributions at Right from Radiological Research Laboratory, Columbia University) 
distributions show the expected agreement. The difference in energy scale reflects a difference in calibration techniques. The Columbia group uses the stopping power of monoenergetic a1pha particles to determine the maximum energy lost by protons to the spherical counter. Our energy scale has been made to agree with an experiment recently performed in our laboratory $(21)$ that measures the maximum energy lost (ionization) by protons passing through the gas used in the spherical counter. This experiment indicates that the maximum energy a proton can lose to a $1 \mu$ site is about $100 \mathrm{KeV}$.

If one makes certain assumptions regarding the behavior of the ionizing events in the counter, the instrument can be used to determine experimentally the dose distribution in linear energy transfer. Figure 33 shows an experimentally measured dose distribution in linear energy transfer for a Pu-Be neutron source (Hanford source \#M-1221), compared with a theoretical dose dis tribution for neutrons from Pu-Be sources The curves have been normalized to equal areas and maximum linear energy transfer of $100 \mathrm{keV} / \mu$.

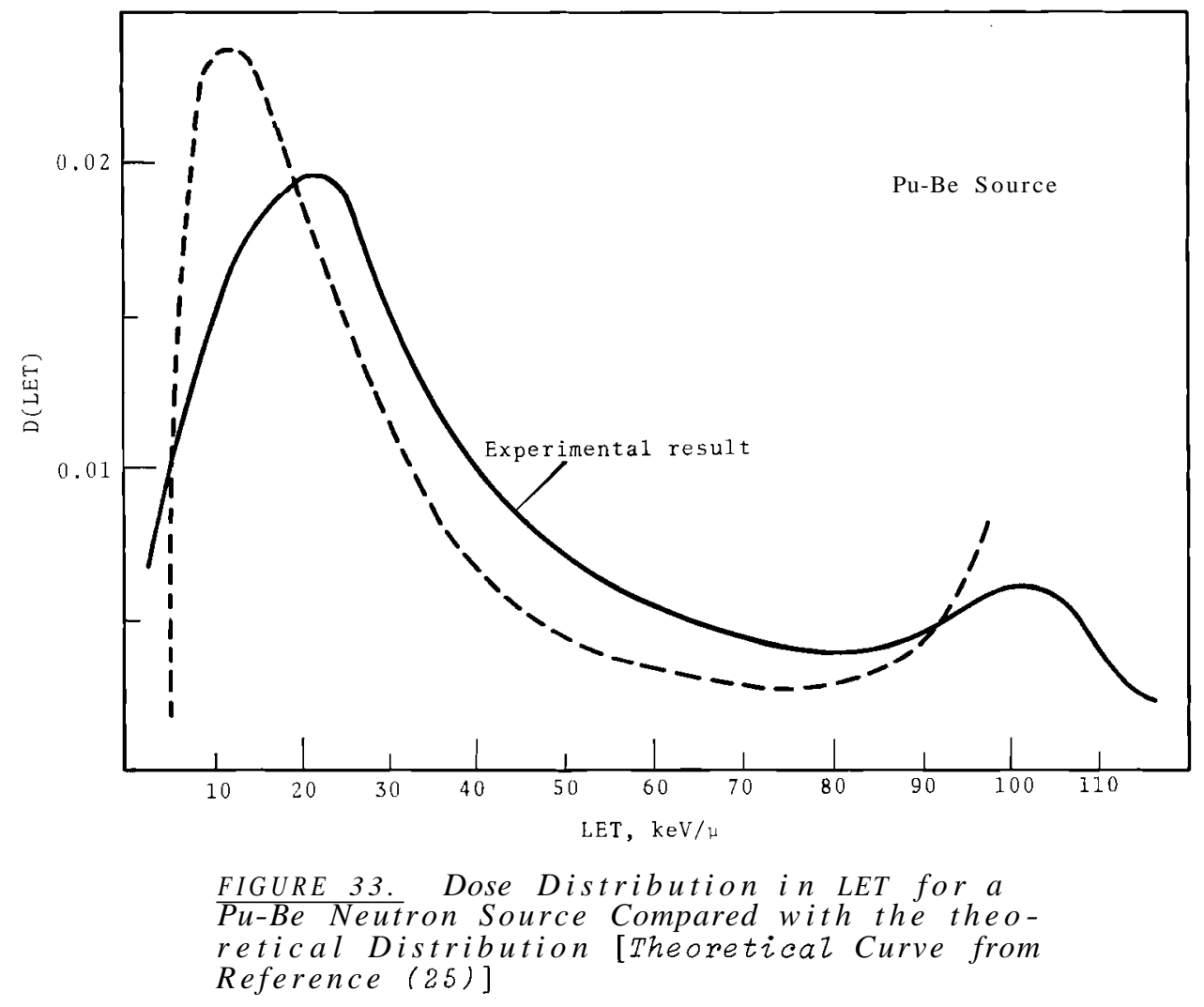

PROGRESS IN ABSORBED-DOSE CALORIMETRY-D. M. Fleming

Calorimetry studies were continued with the goal of developing a calorimeter for use in the measurement of absorbed neutron dose. The dose rate which must be measured is about $1 \mathrm{rad} / \mathrm{hr}$ which is extremely low for calorimetric techniques. If isothermal calorimetry is employed and the measurement extended 
over an 8 hr period, accurate measurement of this dose rate will require the ability to detect temperature changes as low as $10^{-7}{ }^{o} \mathrm{C}$. Extreme temperature stability of the calorimeter will also be required for the $8 \mathrm{hr}$ experiment. Calculations indicate that the required temperature stability and sensitivity may be achieved by using a twin calorimeter.

An important characteristic of the twin calorimeter is the reduced dependence on temperature stability, because temperature changes in the thermal shield affect each cell identically in the ideal case. Also, the twin calorimeter technique is not dependent upon an absolute temperature measurement but reuires only differential measurement which is much easier to obtain.

To apply the twin calorimeter to the measurement of absorbed dose, some means must be incorporated to differentiate between the dose supplied to each side The technique in use is to provide a mass difference between the two absorbers. The outsides of the absorbers are identical to provide the same heat transfer properties, but one is hollow. The absorber with the lower mass absorbs less radiation and as a result has a lower temperature rise.

As a preliminary to the neutron calorimetry, a $\gamma$-ray calorimeter (Figure 34) is partially assembled. This calorimeter will be used as a tool to develop the sensitivity and stability desired for the neutron calorimeter.

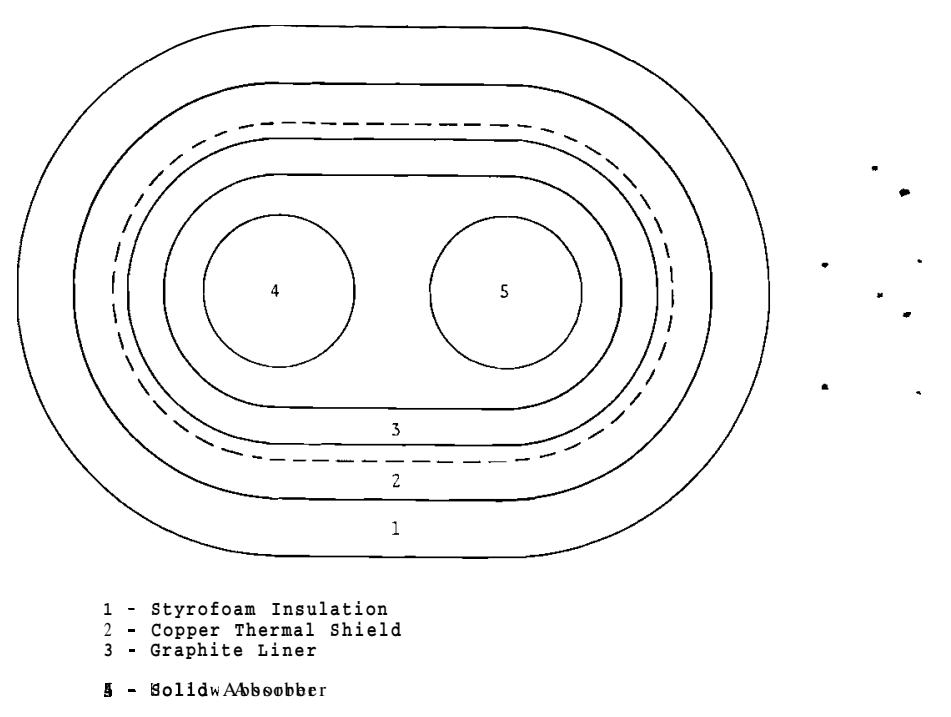

FIGURE 34. Absorbed Dose CaIorimeter with Lids Removed

Later this technique will be adapted for use with neutrons.

Studies are also in progress in the area of sensitive temperature measurement. Although several thermometry systems are available which may be pushed to the $1 \mathrm{imit}$ of $10^{-7}{ }^{\circ} \mathrm{C}$, most have serious drawbacks. Of the several systems that have been studied, thermoelectric materials and gas thermometry show promise of greater sensitivity.

\section{FAST NEUTRON MEDICAL RESEARCH FACILITY - K. L. Swinth}

A shield and irradiation facility were set of for the University of Washington medical research laboratory in Walla Walla. Major modifications to the shielding were made by replacing paraffin with water tanks and concrete bricks. A periscope and ventilation tunnel were included in the shield to alleviate the feeling of closeness for the 
subject. An uneven floor made necessary a slight modification of the tracks on which the bed, spacer, and neutron generator move.

After optimization of the performance of the neutron generator, an estimate of the average dose rate available to the volume of interest using the $D(d, n)$ reaction was made with air-filled, tissue-equivalent, ionization chambers. The average dose rate was found to be $1.2 \mathrm{rads} / \mathrm{hr}$. Similar chambers, but flushed with tissue-equivalent gas were designed and are being assembled (22). Although the $T(d, n)$ reaction will be used for most experiments, the effects of neutrons of lower energy from the $D(d, n)$ reaction are being investigated because of their greater biological interest.

TISSUE EQUIVALENT ION CHAMBER DEVELOPMENT - L. A. Braby

The desirability of tissue equivalence for absorbed-dose measuring devices is well known. In addition, a gamma-only device is needed when mixed fields are encountered. The A150 tissue equivalent plastic(23) was chosen for total dose measurements and graphite for the gamma determinations.

The program needs for ion chambers are a miniature chamber to be used with a pulse pencil reader (24) for depth-dose studies and a small ion chamber for neutron dosimetry to assist the University of Washington spermatogenesis studies.

Since this latter project requires dose distribution measurements and because tissue equivalent gas filling would be inconvenient, a miniature chamber with a small cavity and thick walls was designed. When a desired sensitivity $D /\left(V_{0}-V_{1}\right)$ in rads per volt, has been determined, the necessary ratio of volume to capacitance can be calculated from

$$
Q=f q v=f \frac{e}{w 1.6 \times 10^{-17}} D \frac{(S / \rho)}{(S / \rho)_{1}^{2}} \rho v
$$

and

$$
Q=c\left(V_{0}-V_{1}\right)
$$

where $f$ is the collection efficiency, $q$ is the charge produced in coulombs per $\mathrm{cm}^{3}$ of air, e is the charge of an electron in coulombs. D is the dose in rads, $\frac{(S / \rho)_{1}}{(S / \rho)_{2}}$ is the stopping power ratio of air to wall material, $w$ is the energy in electron volts absorbed per ion pair, p is the density of air, c is the chamber capacitance, and $V_{0}-V_{1}$ is the change in potential on the chamber due to the charge collected.

For the desired V/c value a convenient combination of $r_{2}$, the inner radius of the cavity, and $r_{1}$, the radius of the electrode, can be obtained from

$c=2 \pi \varepsilon_{0} \frac{r_{1} r_{2}}{r_{2}-r_{1}}+2 \pi \varepsilon_{o} L / \ln \left(r_{2} / r_{1}\right)$

$v=2 / 3 \pi\left(r_{2} 3-r_{1}^{3}\right)+\pi\left(r_{2} 2-r_{1}^{2}\right) L$

by successive approximation where L 
is the length of a cylindrical cavity with one hemispherical end. The Type A chamber (Figure 35) was designed for $4 \mathrm{pF}$ and $0.5 \mathrm{rad} / \mathrm{V}$. It s total volume is $18.5 \mathrm{~mm}^{3}$ and $r_{1}$ is $1.375 \mathrm{~mm}$.

Experience with machining and cleaning small insulators soon indicated that Teflon was the best choice, unless polystyrene could be molded to the finished shape. Normally, a new insulator leaks quite badly until it has been cleaned several times. In small chambers dust particles in the gap between electrodes is also a
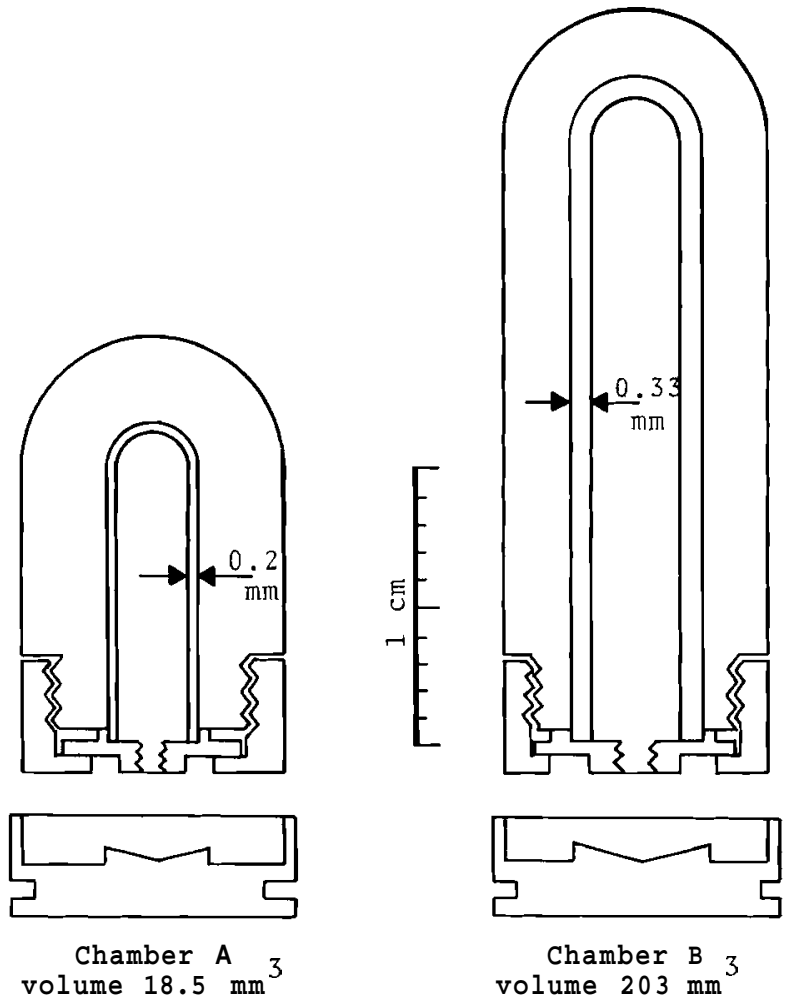

Chamber A volume $18.5 \mathrm{~mm}^{3}$ in gas volumes other than the intended cavity. Even the small air gaps intended to lengthen the surface leakage path over insulators can cause major errors. The chamber contacts, if left uncovered, can collect ions from a very large volume. The configuration illustrated for the A and $B$ chambers proved to be adequate.

In the spermatogenesis project the need arose for more sensitivity and the Type B chambers resulted. These have a sensitivity of $7 \mathrm{~V} / \mathrm{rad}$ (as determined from neutron flux measurements) which is somewhat less than expected. To improve sensitivity without increasing size, these chambers will have to be adapted to the pulse pencil reader.

A $100 \mathrm{~cm}^{3}$ flow chamber using tissue equivalent gas and with $6.5 \mathrm{~mm}$ tissue equivalent plastic walls was designed for calibrating the small chambers. In this chamber,

(Figure 36) insulation is provided by

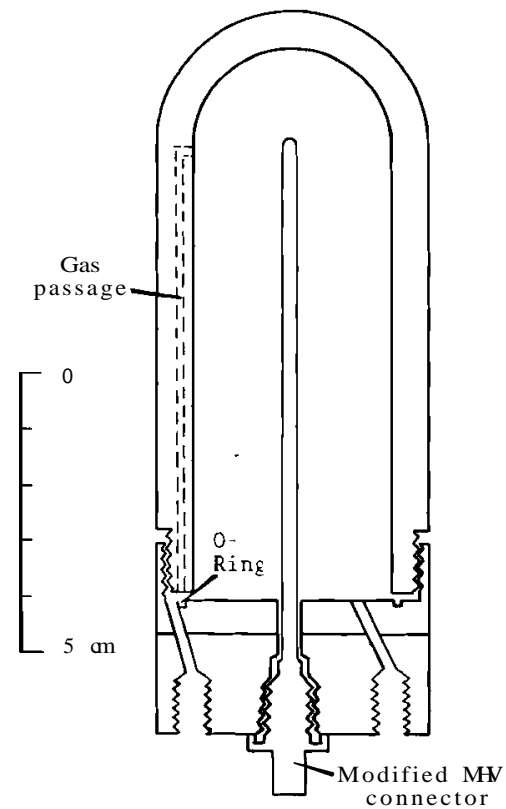

$$
\begin{gathered}
\text { Chamber } \mathrm{M} \\
\text { volume } 100 \mathrm{~cm}^{3}
\end{gathered}
$$

FIGURE 36. Calibrating Chamber Employing Tissue Equivalence and Gas Flow 
a modified MHV connector and leakage resistance is $>10^{16} \Omega$. Chamber current is measured with a vacuum tube electrometer.

CALCULATION OF THE DISTRIBUTION OF DOSE IN LINEAR ENERGY TRANSFER FROM SPHERICAL PROPORTIONAL COUNTER DATA-D. N. Samsky and W. A. GIas S

\begin{abstract}
A computer program was written to calculate neutron dose distributions in linear energy transfer from spherical proportional counter data. A flow diagram is given and sample calculations for several monoenergetic neutron energies are presented. Comparisons with theoretical calculation shows that calculated linear energy transfer distributions are probably valid for neutrons having energies greater than $1 \mathrm{MeV}$.
\end{abstract}

In radiation fields where the charged particles have greatly different linear energy transfers it is difficult to assign a dose equivalent. For radiation protection purposes it would be desirable to measure the absorbed dose for each linear energy transfer, multiply by the corresponding quality factor, and sum the products to arrive at a dose equivalent. Approximate methods have been developed for determining $D(L)$, the dose distribution in linear energy transfer. (25) This method requires knowledge of $N(Y)$, the eventsize distribution. Event size is related to the response observed in a spherical proportional counter and is defined as the energy lost by an ionizing event in a site divided by the equivalent diameter of that site. Then, if it is assumed that the secondaries comprising the events cross the sohere in straight lines, along which their linear energy transfer is constant and, if secondaries starting or stopping in the counter can be neglected,

$$
D(L)=\frac{K}{2 \pi r^{2}}\left[Y N(Y)-Y^{2} \frac{d N(Y)}{d Y}\right]_{Y=L}
$$

where $r$ is the equivalent radius of the sphere. These assumptions are reasonably well satisfied for neutrons above about $1.0 \mathrm{MeV}$, high energy charged particle beams, etc. I t is convenient to note that $D(Y) \propto Y N(Y)$; then

$$
\begin{aligned}
D(L)= & \frac{K^{\prime}}{2 \pi r^{2}} \\
& {\left[2 D(Y)-Y \frac{d D(Y)}{d Y}\right]_{Y=L} }
\end{aligned}
$$

The data for $N(Y)$ are obtained as a histogram pulse-height distribution from a multichannel analyzer. A computer program was written to produce $D(L)$ from such pulse-height spectra.

The program was written in Fortran IV for the Univac 1107 computer. Figure 37 is a brief flow diagram of the code. The necessary input parameters, in addition to the $N(Y)$ distribution, are the counter 


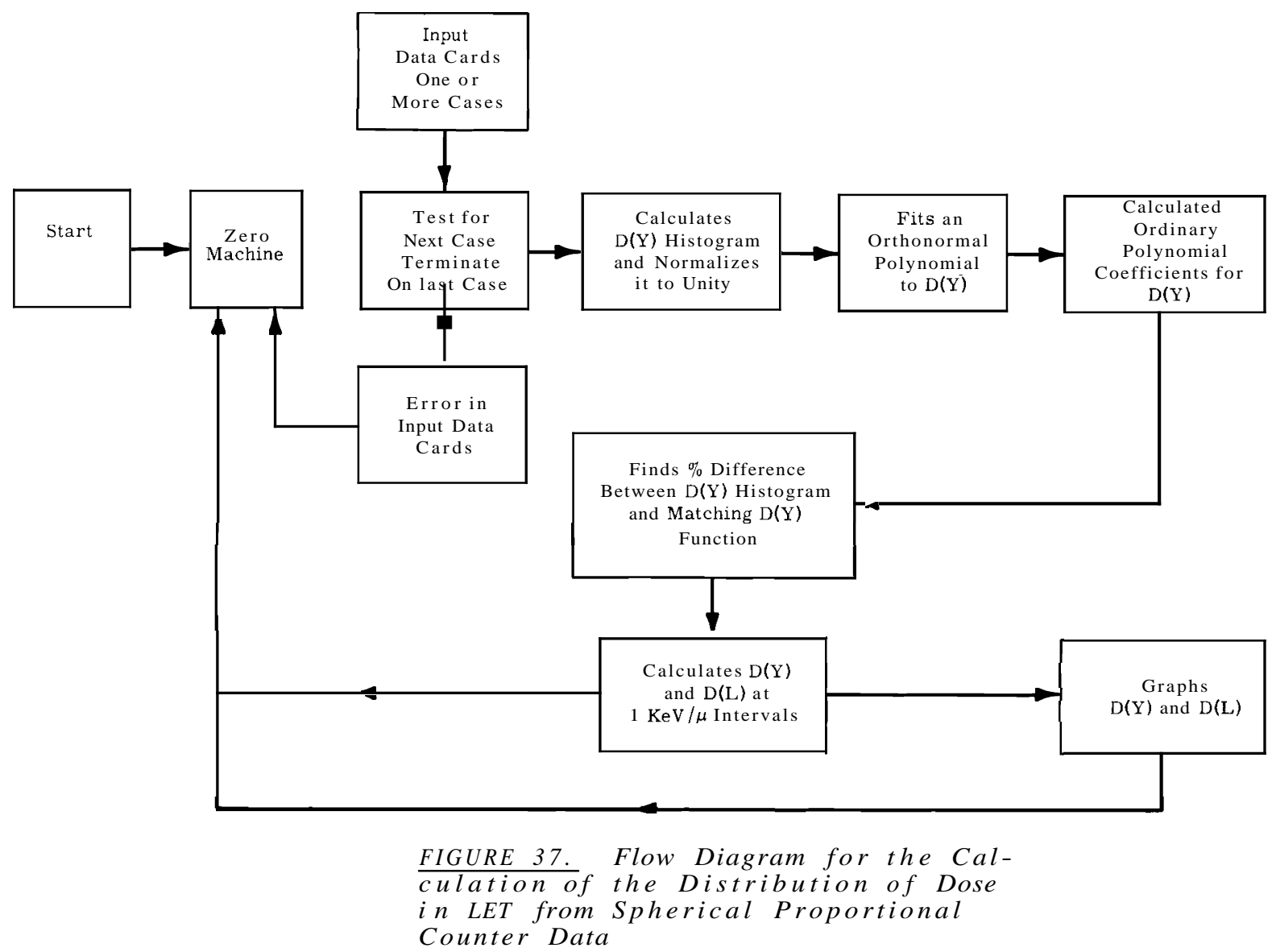

size and input energy calibration.

The date, run number, energy and any other additional information can be associated with each computation. A plotting option is available so that either $D(L)$ or $D(Y)$ and $D(L)$ are graphed. Only the statistically significant data of the multichannel analyzer output were included for analysis.

The input was the Hanford standard LISTIV subroutine. First a D(Y) histogram was calculated from the data and normalized to unity. This calculation as well as all others, was performed in double precision so that each number had 21 significant digits thus insuring that round off errors in divisions and subtractions would not affect the results. A set of orthonormal polynomials was then found to represent the normalized $D(Y)$ histogram. The order of the polynomial, less than 16, was chosen to minimize the residue. From this, a set of ordinary polynomial coefficients was constructed. A point by point comparison between the $D(Y)$ histogram and the calculated fit reflected no more than the statistical fluctuation of the data. Adjustments were made so that computer calculations of the $D(Y)$ and $D(L)$ function were made at $1 \mathrm{keV} / \mu$ intervals. The code returns the normalized $D(Y)$ histogram, the matching $D(Y)$ function, the percent difference between the two, the ordinary polynomial coefficients, and the adjusted $D(Y)$ and $D(L)$ functions. In addition it can supply plots for $D(Y)$ and $D(L)$. 
The operation of the code was checked by comparison with a few points calculated by hand and was satisfactory. Figures 38 and 39 show the results of representative cases that have been normalized so that the high linear energy transfer peak in the $D(L)$ spectrum falls in Channel 100. It should be noted that even though results for $0.5 \mathrm{MeV}$ neutrons have been presented, the $D(L)$ distribution is not correct due to the high percentage of secondaries stopping within the counter. It has been included to show that its general shape above $40 \mathrm{keV} / \mu$ is similar to that from the theoretical calcu- lations by Rossi. (25) A comparison of the N(Y) distribution with the theoretical distribution calculated by Caswell(26) is shown in Figure 40. I t shows that for neutron energies above $1.0 \mathrm{MeV}$ a $\mathrm{D}(\mathrm{L})$ distribution can be constructed. A D (Y) distribution (Figure 41a) for a Pu-Be neutron source indicates that it would be correct to calculate a $D(L)$ distribution (Figure 41b). If the accepted values for quality factors at various linear energy transfer values (Handbook 59) are used, a quality factor of 7 can be assigned to the neutrons from a $\mathrm{Pu}-\mathrm{Be}$ neutron source on the basis of its linear energy transfer dose distribution.

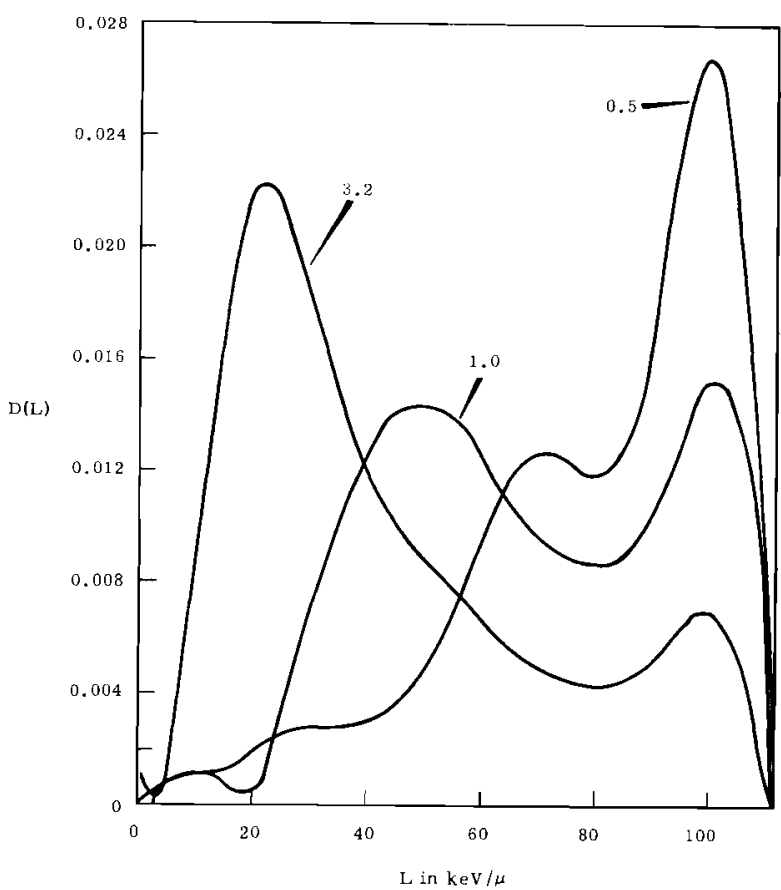

FIGURE 39. Dose Distribution in LET for Monoenergetic Neutrons 

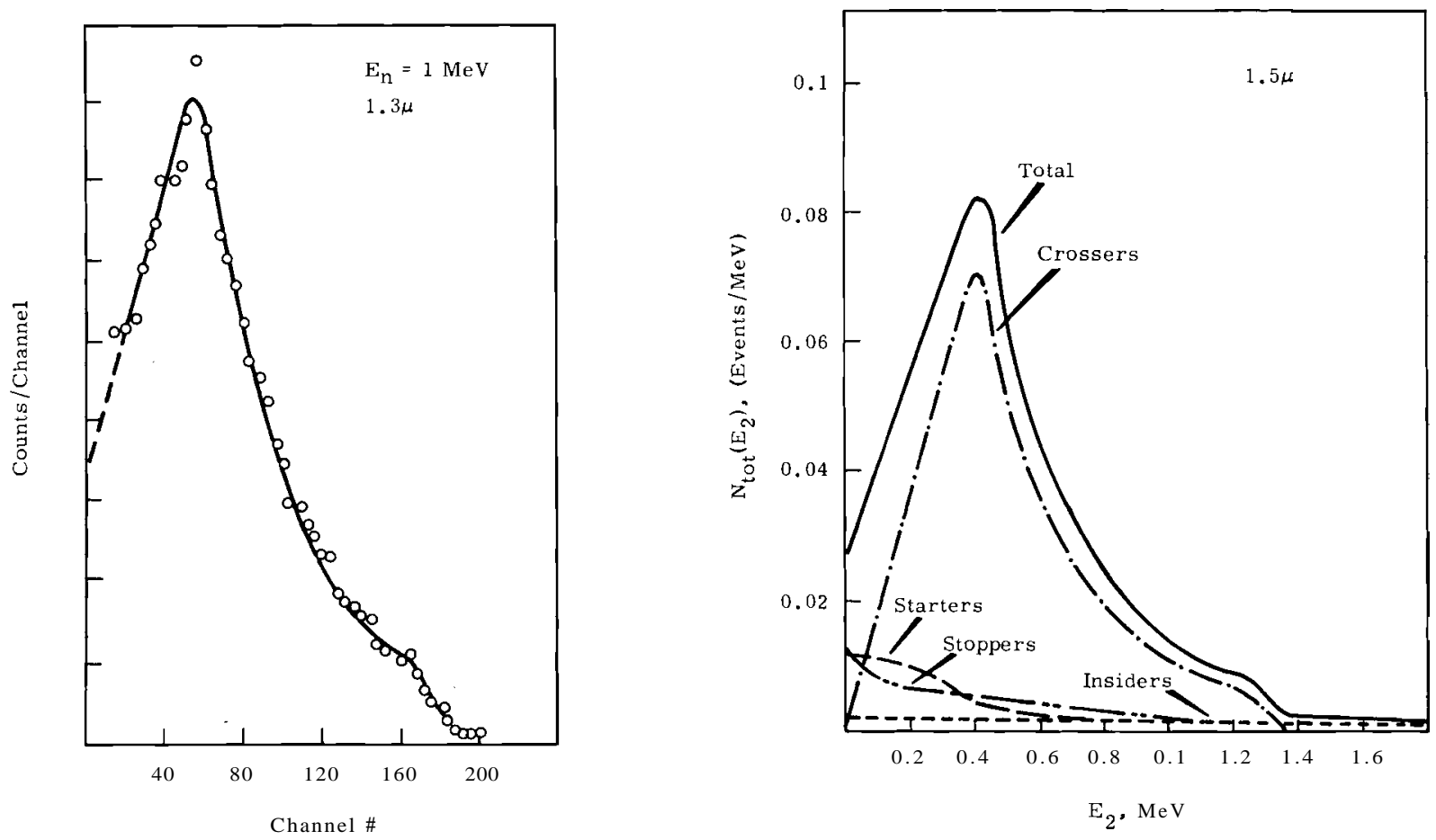

FIGURE $40 \mathrm{fa}) . \quad N(Y)$ Distribution from Spherical Proportional Counter Data

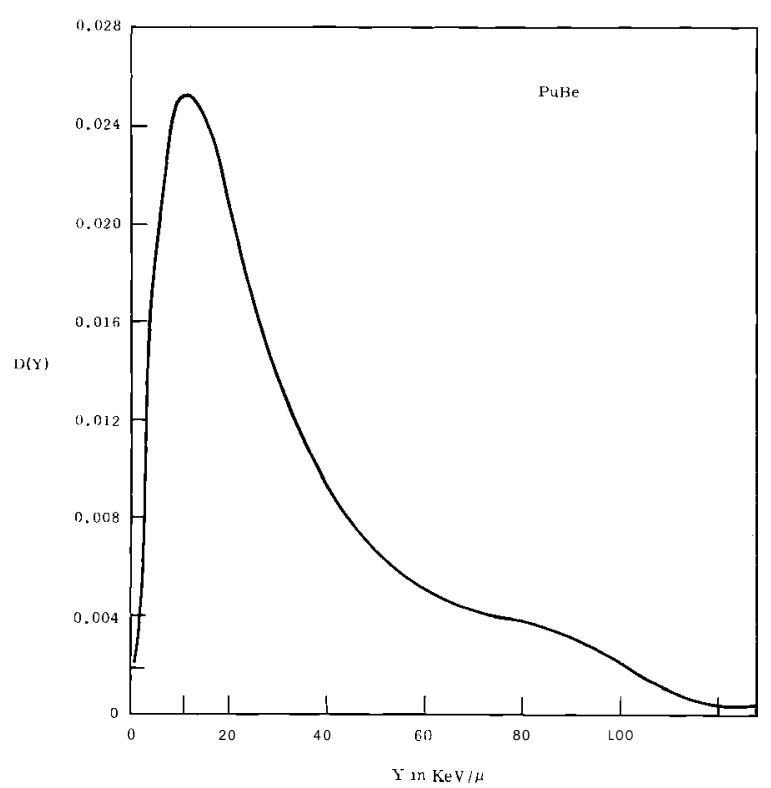

FIGURE 40 (b). N(Y) Distribution from Caswe zl's Calculation

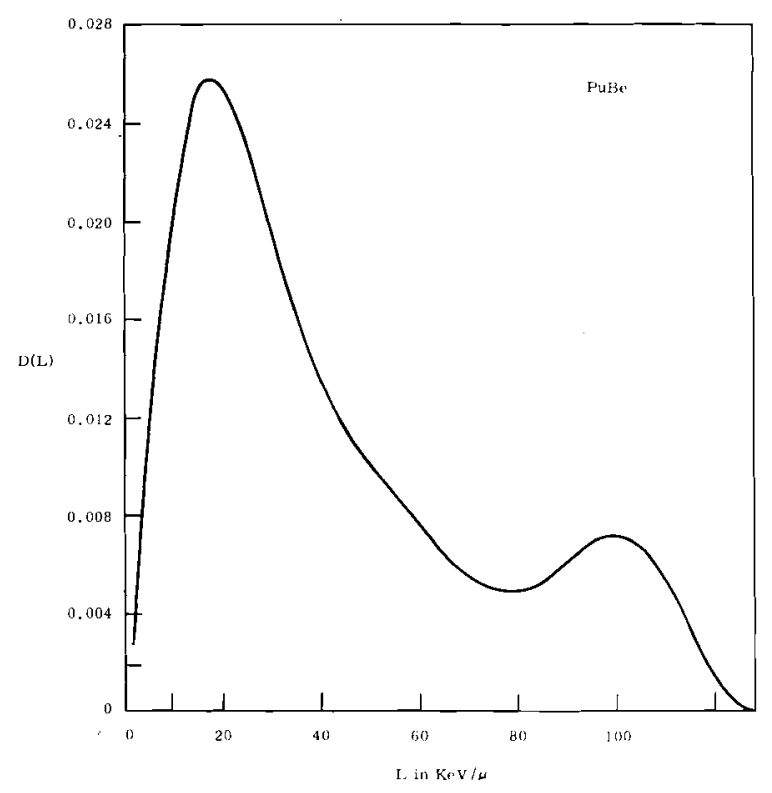

FIGURE $41 \mathrm{fa})$. Dose Distribution on Y for a Pu-Be Source

FIGURE 41 (b). Dose Distribution in LET for a Pu-Be Source 
LOW ENERGY FAST NEUTRON SPECTROMETER-W. A. Glass

A $\mathrm{He}^{3}$ - filled proportional counter was developed to aid in the evaluation of neutron spectra in the energy range 0.1 to $1.0 \mathrm{MeV}$. The counter is characterized by its wa11less construction and isotropic response to neutron flux. Resolution is approximately $40 \mathrm{keV}$ f.w.h.m. for $125 \mathrm{keV}$ neutrons.

TRANSITION ZONE DOSIMETRY-K. G. Pailthorp

The distribution of the dose near the interface between a beta-emitting half-space and another medium is influenced by the atomic composition of the backscattering medium as well as that of the emitting medium. For half-spaces with dissimilar atomic compositions, the dose distribution is discontinuous at the interface, a phenomenon known as the stopping power jump, since the discontinuity results from the difference in the mass stopping powers of the materials. In this study, dose distributions were studied for two metallic emitting media in combination with three metallic backscattering media--a total of six distinct cases.

A calibrated solution of ${ }^{32} \mathrm{P}$ was mixed with 250 mesh powders of aluminum and lead. The mixtures were allowed to dry and then compacted on a 300 ton press. (28) The result was two metal disks 3 in. diam and thicker than the maximum range of ${ }^{32} \mathrm{P}$ beta particles. The densities achieved were 100 and $93 \%$ of theoretical density for aluminum and lead respectively.

Aucoradiographs were made of the sources to determine the uniformity of the activity. Figure 42 is a histogram display of optical density readings at a number of random sites in the collecting region of the faces of the emitting disks. The aperture of the optical densitometer used was $1 / 16$ by $3 / 16$ in., so some averaging is inherent in the readings.

The dose rate at the interface for an example in which the two media are of the same atomic composition is equal to one half of the dose rate at the center of an infinite emitting medium, $R_{\infty}$, which is just (29)

$$
R_{\infty}=2.13 \bar{E} C \mathrm{rad} / \mathrm{hr}
$$

where $\overline{\mathrm{E}}$ is the average energy in $\mathrm{MeV}$ of the electrons emitted, and $\mathrm{C}$ is the concentration of emitter in $\mu \mathrm{Ci} / \mathrm{g}$. If the activity was uniformly distributed, the measured interface dose rate would agree with that calculated from the above relationship. The calculated and measured interface dose rates for the aluminum emitter half-space were respectively 10.20 and $10.36 \mathrm{rad} / \mathrm{hr}$. The calculated and measured interface dose rates for the lead emitter half-space were respectively 10.21 and $10.18 \mathrm{rad} / \mathrm{hr}$. The agreement is within the range of the experimental uncertainty. 
BNWL-235 3

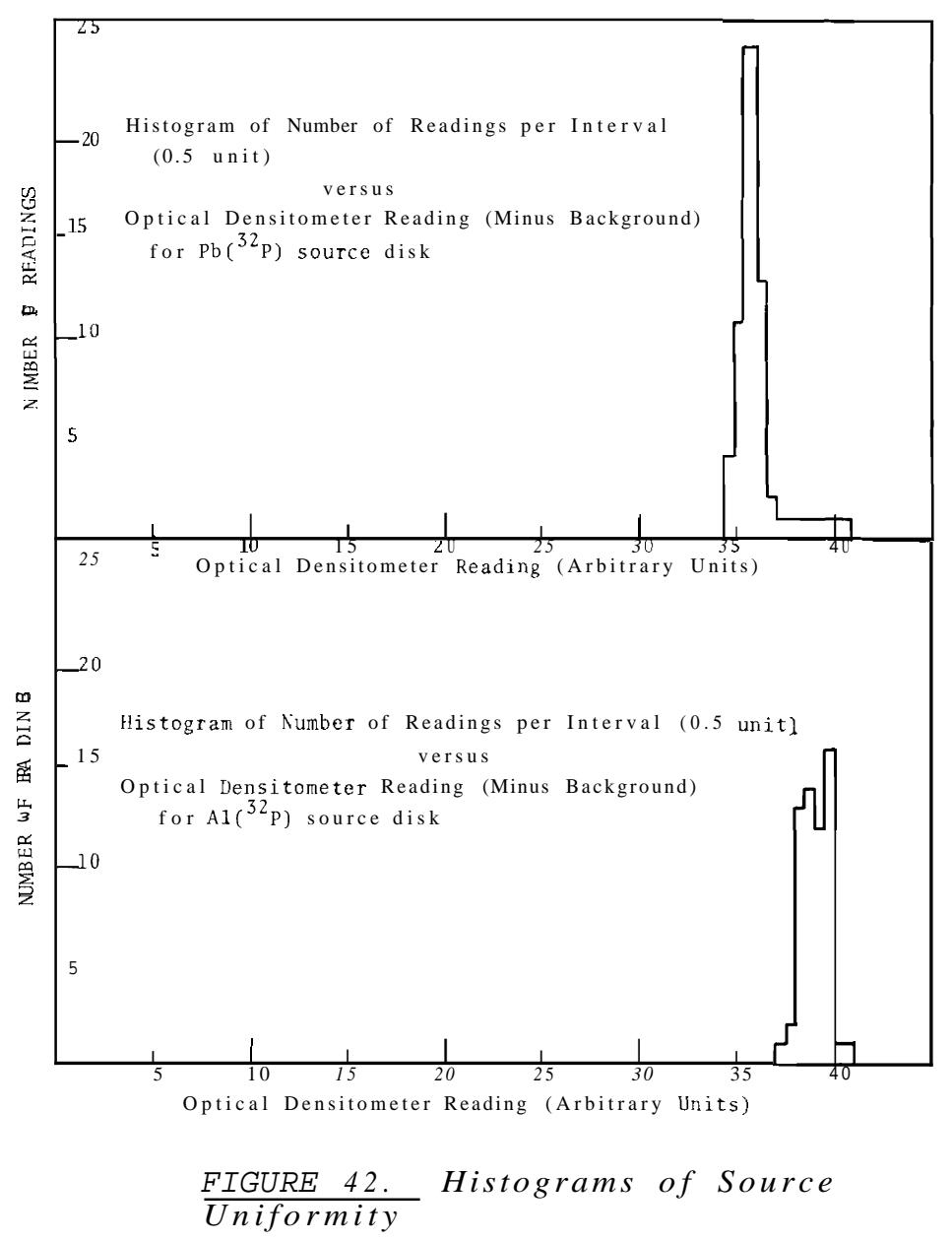

The extrapolation chamber is shown in Figure 43. The emitter half-spaces were mounted to Lucite disks through which extended electrical leads.

This assembly served as the accelerating electrode within the extrapolation chamber.

In opposition to this electrode within the extrapolation chamber was one of three collector-guard ring assemblies--one of aluminum, one of copper, and one of lead. Each assembly consisted of a disk 1 in. diam surrounded by an annulus of $1.010 \mathrm{in}$. ID and 3 in. OD. These were cemented to a disk of cast electrical epoxy resin in such a way as to maintain the 0.005 in. air gap between the collector and the guard ring. The front surface of this assembly was subsequently faced off on a lathe to insure the coplanarity of the collector and guard ring. Electrical leads extending through the resin maintained the guard ring at ground potential and conducted the ionization current from the collecting electrode to the input of a vibrating reed electrometer. The electrometer was used in the rate-ofcharge mode, and the output was displayed on a strip chart recorder.

The entire extrapolation chamber assembly was mounted directly on the 


\section{Metal}

Epoxy
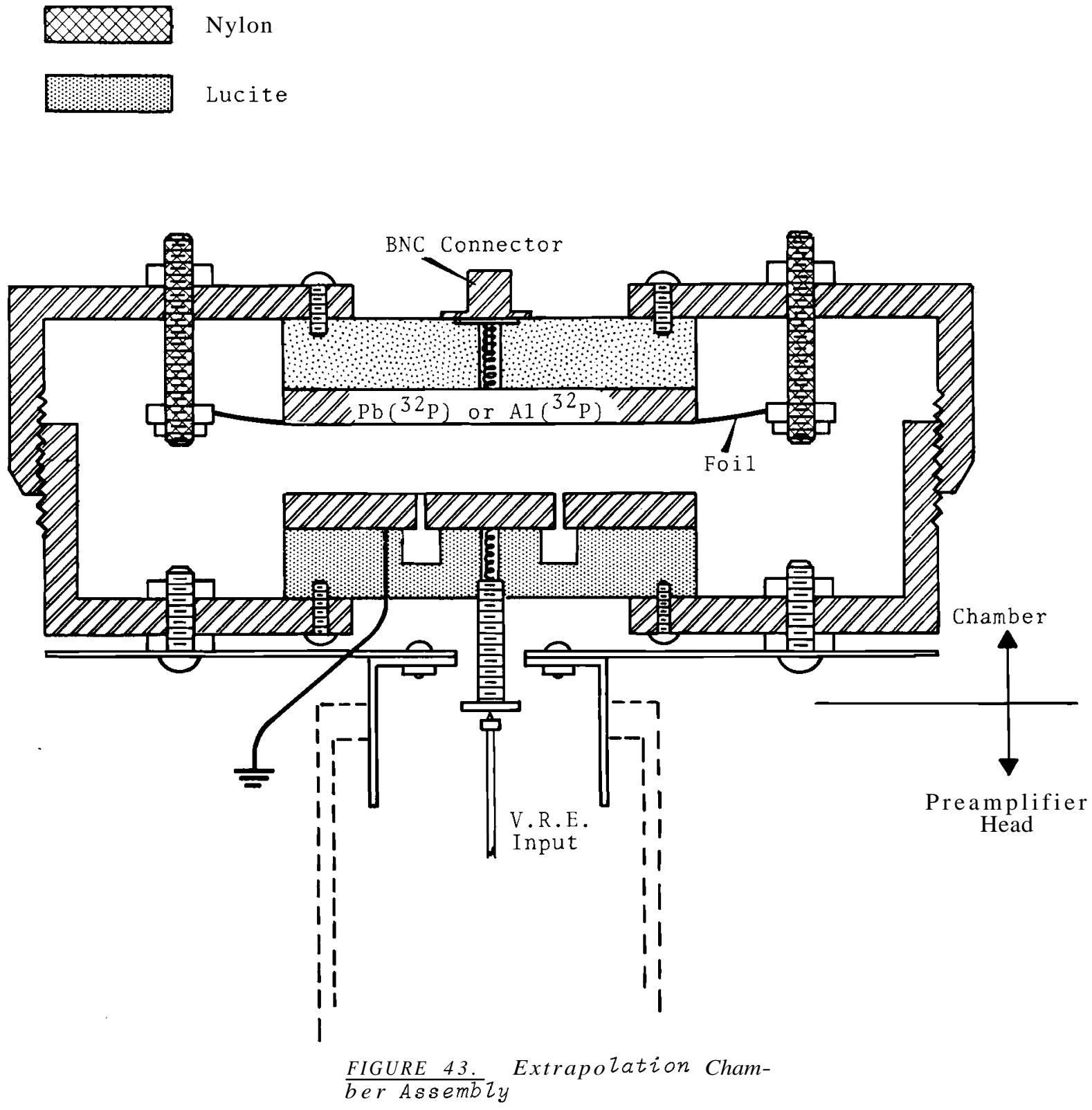

head of the preamplifier unit of the electrometer. The case of the extrapolation chamber consisted of two threaded aluminum cylinders. The threads were $16 /$ in. and the circumference of the outer cylinder was divided into degrees, providing positioning of $1.736 \times 10^{-4}$ in. $/^{\circ}$. An aluminum frame consisting of two annuli was used to clamp foils of aluminum, copper, or lead and to stretch them over the accelerating electrode within the chamber for the purpose of examining the dose rates at positions within the backscattering medium. 
An independent determination was made of the position corresponding zero plate separation. Capacitance was read with an L-C meter as the chamber setting was varied. A plot of (capacitance) ${ }^{-1}$ versus chamber setting gave a straight line which when extrapolated to the abscissa indicated the chamber setting corresponding to zero plate separation.

Six configurations were investigated-three backscattering halfspaces for each of the two emitter half-spaces. The results are shown in graphic form in Figures 44 through 47. The quantity JW plotted in Figures 43 and 44 and referred to as chamber response is related to dose rate through the Bragg-Gray relationship, (30)

$$
\mathrm{R}=\mathrm{sJW}
$$

where $s$ is the ratio of the mass stopping power of the wall material

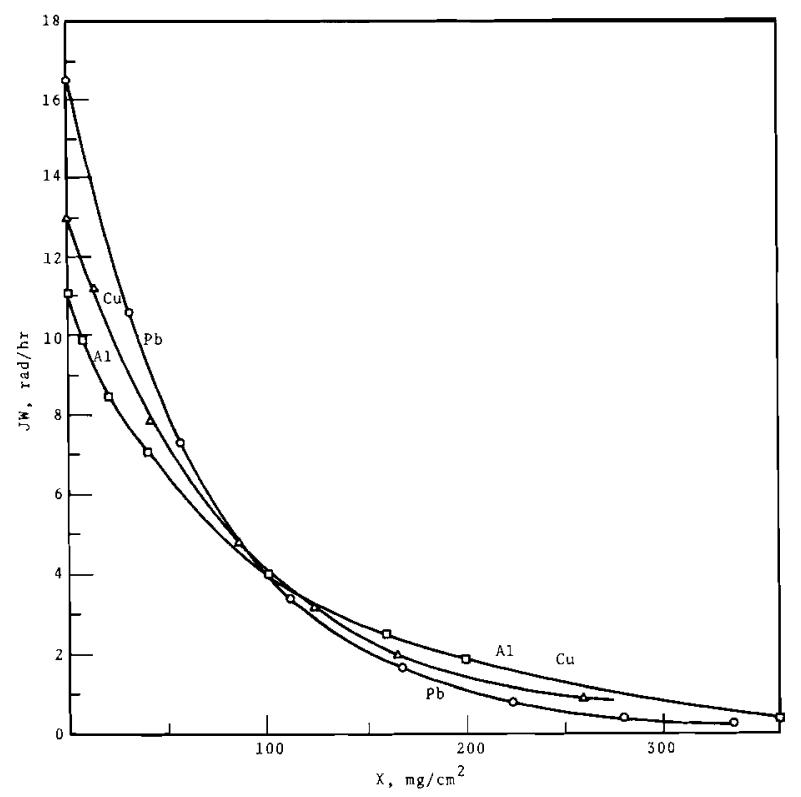

FIGURE 44. Chamber Response for $A \mathcal{L}\left({ }^{2} P\right)$ Emitter Half-Spaces

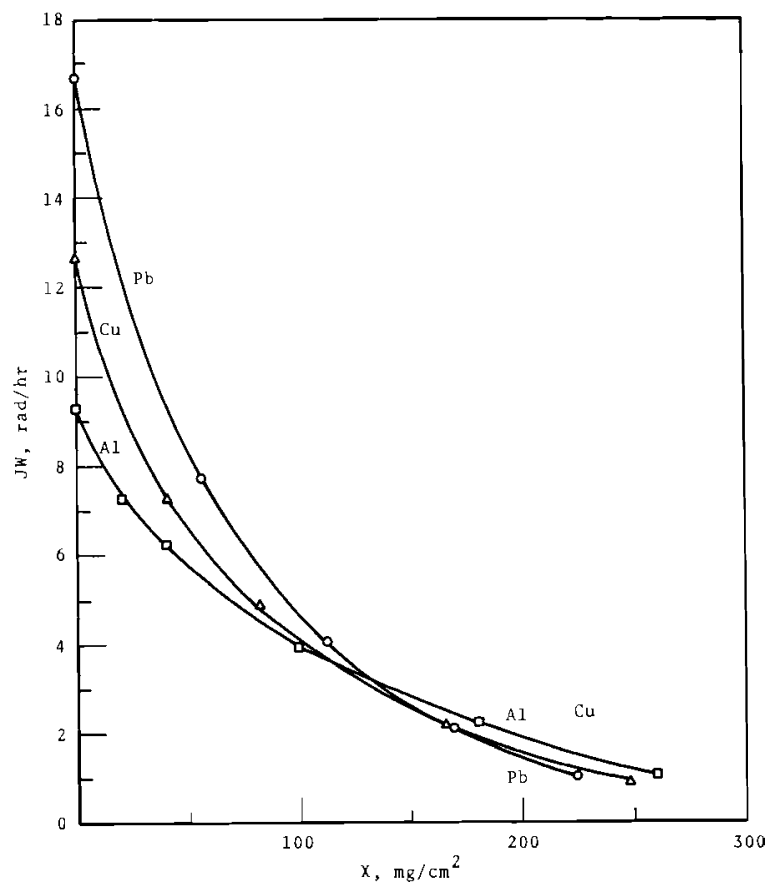

FIGURE 45. Chamber Response for $\mathrm{Pb}(32 \mathrm{P})$ Emitter Half-Spaces

for electrons to that of the gas with in the ionization cavity, and $R$ is the dose rate within the wall material. Note that the chamber response at the interface increases with increasing $Z$ of the backscattering material. This results from the fact that the mean square angle of the scattering is proportional to $(2)^{2}$. That is, more energy is reflected back to the interface. This same consideration accounts for the more rapid decrease with depth $\left(\mathrm{mg} / \mathrm{cm}^{2}\right)$ of chamber response for the higher Z materials which causes the three chamber response curves to cross. Due to the greater range (mg/ $\mathrm{cm}^{2}$ ) of electrons in the higher $\mathrm{Z}$ materials as well as the greater bremsstrahlung production (since the ratio of the energy lost by electrons through radiative processes to that 


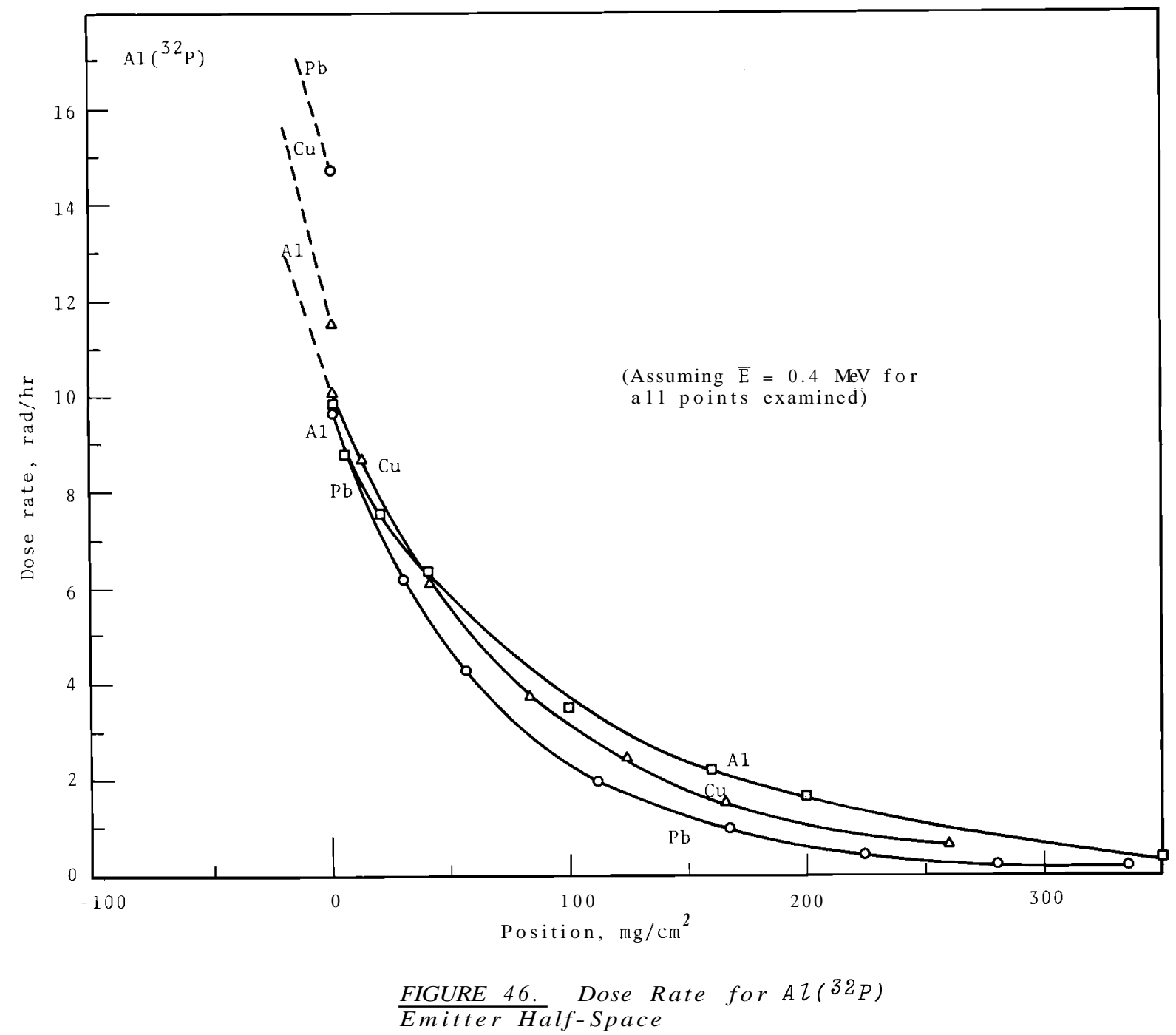

lost in collisions is proportional(32) to $Z$,) the curves will cross again at a depth beyond those attainable with this apparatus.

The values of dose rates plotted in Figures 45 and 46 depend on the stopping power ratios (33) for the materials used and also on the energy spectrum of electrons at a given position, since stopping power ratios are functions, albeit slowly varying, of electron energy. The simplifying assumption adopted in this report is that the average electron energy, $\bar{E}$, was $0.4 \mathrm{MeV}$ at all points in the materials. This is a reasonable approximation in the region of most practical interest (i.e. near the interface); however, the approximation deteriorates as points are considered deeper within the backscattering medium.

When stopping power jumps occur at the interface, two dose rates were obtained from successive applications of the stopping power ratios 


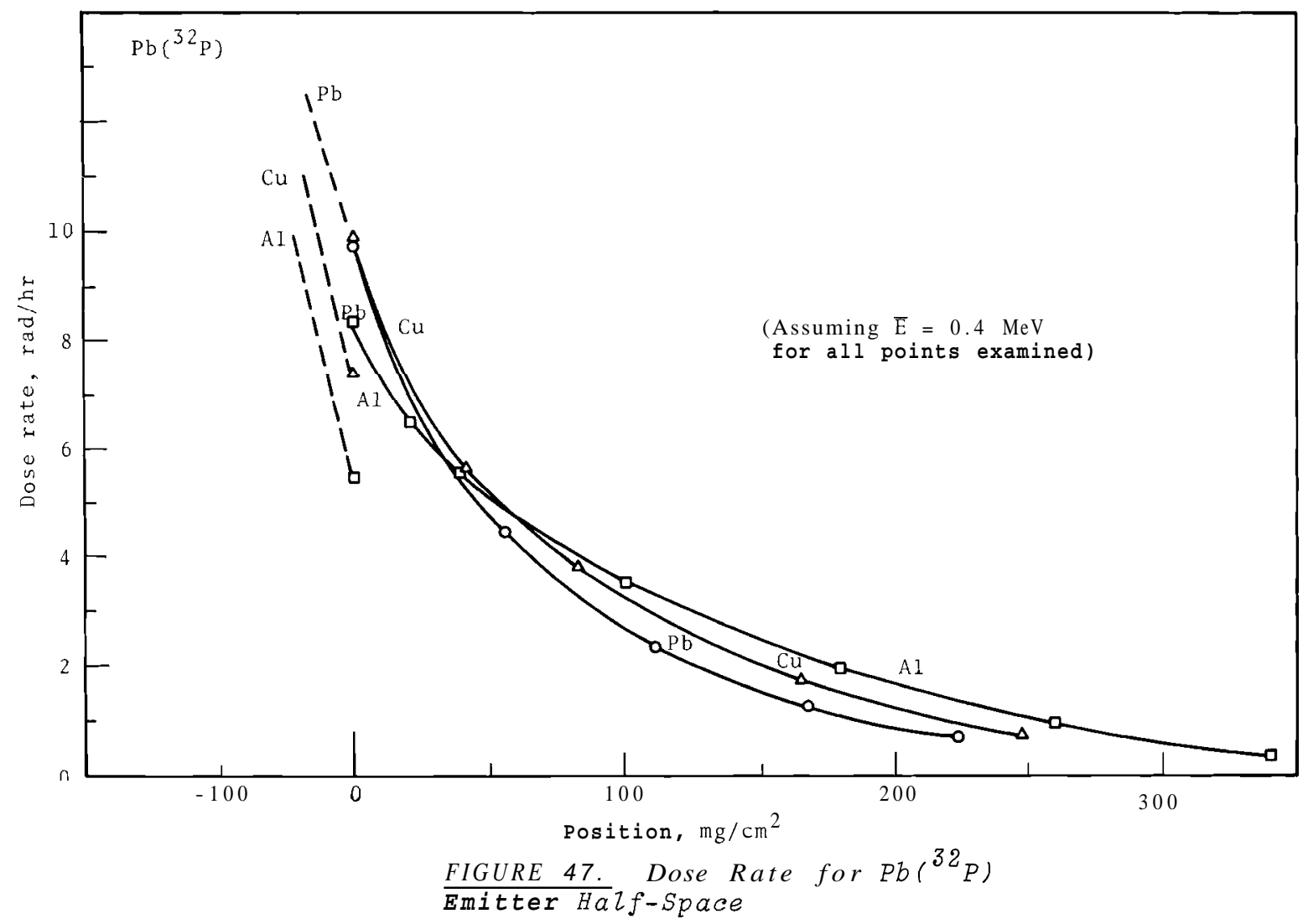

for the two materials to the chamber response reading at the interface.

The dashed curves to the left of the interface in Figures 46 and 47 are intended only to suggest the way in which the dose rate curves approach the interface. No data were taken in this region. However, the data taken in the backscattering medium afford some information about dose rate curves in the emitting medium. Energy conservation dictates that the area under the curve to the right of the interface be equal to the area to the left of the interface bounded above by $2.13 \overline{\mathrm{E} C}$ and below by the dose rate curve. That is, the energy absorbed in the backscattering medium must equal the energy escaping the emitting medium. Also the dose rate curves must converge to 2.13 EC for depths greater than the maximum range of the most energetic electron emitted. These considerations provide an index to the nature of the dose rate curves within the emitting medium. 
WHOLE BODY COUNTING OF PLUTONIUM IN DOGS* K. L. Swinth

Experiments were performed with dogs to learn more about the problems associated with in vivo counting of plutonium. These experiments are a continuation of previous work.

Physical differences in dogs, which affect plutonium counter sensitivity, were further investigated. The effect of the skeleton on counting was determined both by counting sources in a dog skeleton and by measuring rib width and spacing on X-ray films. By measuring width and spacing of ribs on X-ray films of dogs we estimated that $28 \%$ of the chest area was covered by ribs. This value ignores the effect of the scapula, sternum, and spinal column. Counting a source placed at the lymph node location in the skeleton gave a decrease of $46 \%$ from the rates with the source in the same location but without the skeleton.

Previous investigations determined the attenuation of $17 \mathrm{keV}$ photons in the chest wall of different dogs by inserting a plutonium source down the trachea to the bifurcation, thus approximating plutonium in the bronchial lymph nodes.. (34) Combining these results with the skeleton data we obtain a half value layer of $1.3 \mathrm{~cm}$ for $17 \mathrm{keV}$ photons in the tissue of the chest wall.

Sources of the same isotopic composition as the exposure material were obtained and used to study the effects of different distributions in the dog phantom. The changes in sensitivity with distribution are

Work done in cooperation with J.F. Park of the Biology Department. shown in Figure 48. The new sources gave counting rates almost identical to those of exposed dogs. This confirms our earlier prediction that the previously observed difference in sensitivity between phantom and real dogs (34) was due to different plutonium isotopic composition.

Changes in distribution seem to account for the major differences in sensitivity noted with different dogs. Table 2.2 shows the sensitivities obtained for different dogs compared with those predicted from the phantom. the determination of the body burden and the real distribution within the dog were obtained by alpha counting of the tissues.

If one assumes that counting rates of background dogs and contaminated dogs are normally distributed statistically, one can state a lower limit for detection of plutonium contamination. The background counting rate as determined from 20 uncontaminated dogs was $116 \pm 10.7$ (standard deviation of the counts) counts/min for the $17 \mathrm{keV} X$-ray region and $607 \pm 53$ counts/min when the $60 \mathrm{keV}$ gamma ray was included. The sensitivities determined from the eight dogs in Table II were $2.28 \pm 0.16$ and $13.28 \pm 1.09$ counts/min-nCi respective energy regions. With a $5 \%$ chance of judging a background animal contaminated and a $50 \%$ chance of calling a dog with the limiting 


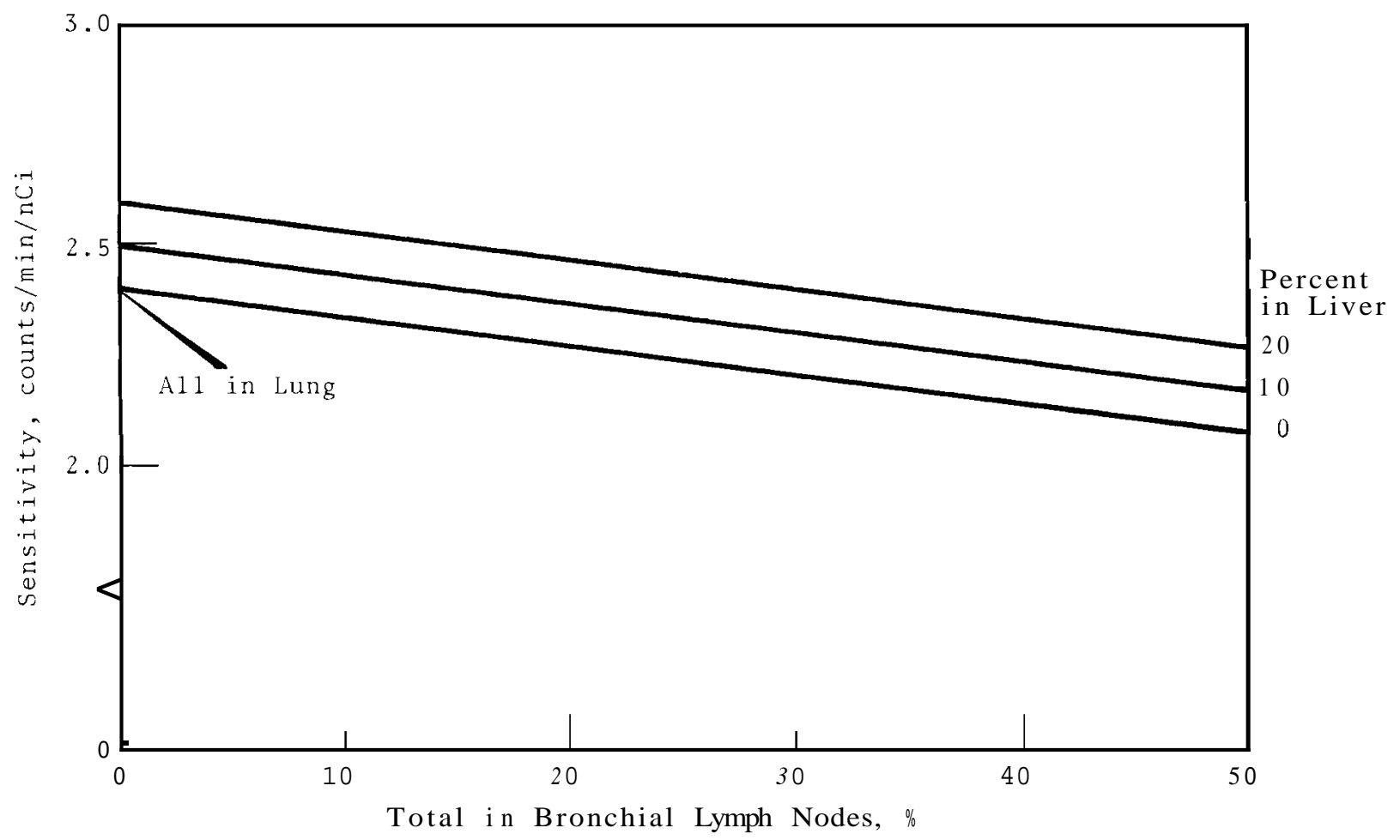

FIGURE 48. Sensitivity of Plutonium

Whole Body Counter for Different

Distributions of Activity

amount uncontaminated, the lower

$1 \mathrm{imit}$ for the $\mathrm{X}$ ray region is $7.7 \mathrm{nCi}$, and for the $17 \mathrm{keV} X$ ray plus $60 \mathrm{KeV}$ gamma the $1 \mathrm{imit}$ is $6.6 \mathrm{nCi}$. This is the "minimum significant measured activity" in the notation of Altshuler and Pasternack(35) if we assume that the background rate is well known. If we reduce the possibility of calling a contaminated dog uncontaminated (Type I error) from 50\% to $5 \%$ ("minimum detectable true activity"(35)),

\begin{tabular}{|c|c|c|c|c|c|c|c|}
\hline \multirow{4}{*}{$\begin{array}{l}\text { TABLE } \\
\underline{\text { Dog }}\end{array}$} & \multirow[t]{3}{*}{$I I$} & \multirow{2}{*}{$\begin{array}{l}\text { Effect of Organ } \\
\text { Percent Plutonimm }\end{array}$} & \multirow{2}{*}{\multicolumn{2}{|c|}{$\begin{array}{l}\text { Distribution } \\
\text { in Tissue }\end{array}$}} & \multirow{2}{*}{ on } & \multicolumn{2}{|c|}{ Calibration } \\
\hline & & & & & & \multicolumn{2}{|c|}{ Sensitivity, counts/min-nCi } \\
\hline & & Bronchial & & & & & Predicted \\
\hline & Lung & Lymph Nodes & $\underline{\text { Liver }}$ & Other & & Measured & From Phantom \\
\hline 76 & 47 & 45 & 3 & 5 & & 2.1 & 2.14 \\
\hline 183 & 52 & 38 & 6 & 4 & & 2.1 & 2.19 \\
\hline 213 & 49 & 27 & 15 & 8 & & 2.3 & 2.35 \\
\hline 272 & 63 & 17 & 14 & 6 & & 2.45 & 2.42 \\
\hline 85 & 36 & 30 & 21 & 13 & & 2.41 & 2.38 \\
\hline 216 & 51 & 26 & 15 & 8 & & 2.40 & 2.35 \\
\hline 257 & 41 & 32 & 21 & 6 & & 2.02 & 2.37 \\
\hline 268 & 43 & 26 & 21 & 10 & & 2.47 & 2.40 \\
\hline
\end{tabular}


we get 17.3 and $13.5 \mathrm{nCi}$ for the respective energy groups.

A means of predicting the sensitivity would be valuable in increasing the accuracy of our counting results. One easily obtained variable that is sensitive to changes in the internal distribution of plutonium is the ratio of the counts in the X-ray channels to those in the $X$-ray channels plus those in the $60 \mathrm{keV}$ gamma ray channels, $\left(\begin{array}{llllll}\sum \text { counts, } & \text { channels } & 13.6 & \text { to } & 25.6 & \mathrm{keV} \\ \sum \text { counts, } & \text { channels } & 13.6 & \text { to } & 66.4 & \mathrm{keV}\end{array}\right)$. Results obtained for sensitivity versus ratio with the phantom are shown in Figure 49. This ratio depends on the plutonium isotopic composition and the position of the dog in the counter in addition to the distribution of the plutonium within the dog. The physical characteris tics of the dog can also affect this relationship. Adding fat or tissue pads to the phantom produced a parallel but lower line. A similar relation is found for the $17 \mathrm{keV}$ $X$ ray plus $60 \mathrm{keV}$ gamma versus the ratio.

The feasibility of counting tissues from sacrificed dogs has been explored. Counting thin sections ( $3 \mathrm{~mm}$ ) inclosed in plastic bags and placed on top of one counting unit gave good agreement with the results from alpha counting. Two standard deviations of the background rate correspond to about $0.33 \mathrm{nCi}$. Sandwiching the samples between two units should provide greater sensitivity than the 23 counts/min-nCi obtained.

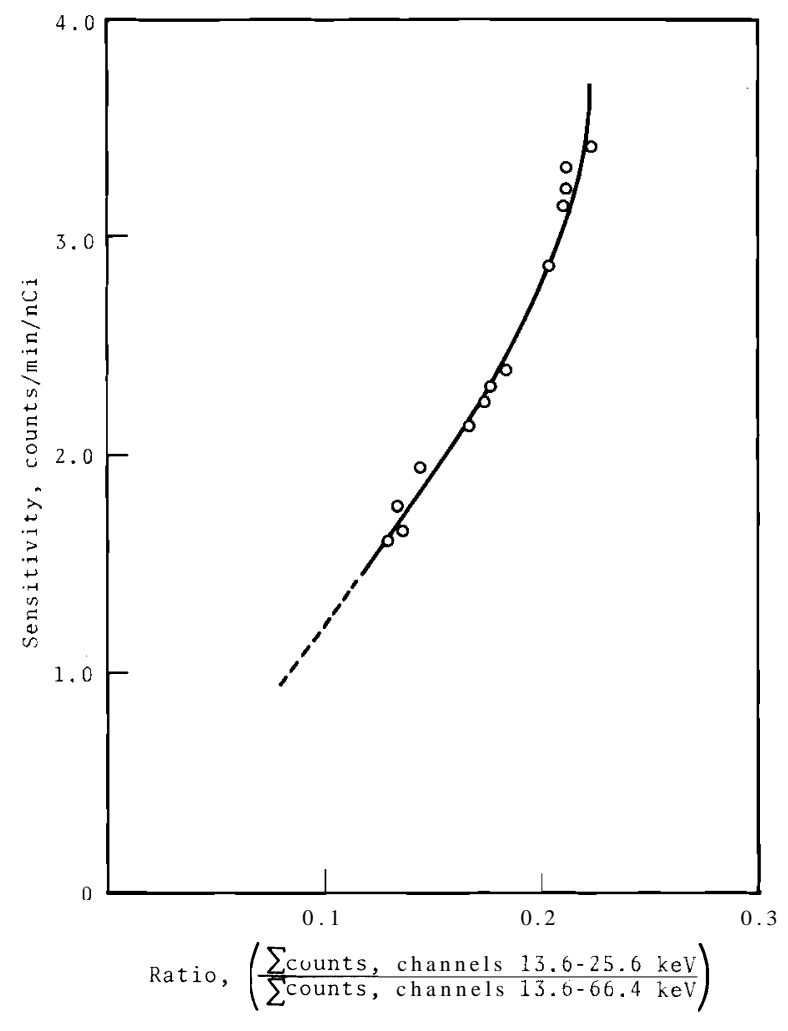

FIGURE 49. Sensitivity in X-Ray Ratio for Dog Phantom in Plutonium Counter 
SHORT-TERM RETENTION OF PLUTONIUM IN DOGS* $-K$. L. Swinth

The short term retention of plutonium in dogs was studied by whole body counting. A half-time for retention of about 1 day was obtained for the rapidly eliminated portion.

After an exposure to plutonium, a large fraction of the initially deposited material is eliminated by the body. Recently 13 dogs were exposed to four differently-prepared plutonium oxides and the retention of plutonium studied by whole body counting.

The dogs inhaled aerosols produced with a vibrating type generator containing dry plutonium oxide dust. The oxides were prepared in four different manners from two isotopically different plutonium sources:

- Oxalate calcined at $300^{\circ} \mathrm{C}$ (Isotope A)

-Above recalcined at $1000^{\circ} \mathrm{C}$ (Isotope A)

@Deltaform of plutonium metal oxidized at $123^{\circ} \mathrm{C}$ (Isotope B)

@ Delta form of plutonium metal oxidized at $450^{\circ} \mathrm{C}$ (Isotope B)

After the dogs were exposed to the plutonium aerosol, they were cleaned of contamination, wrapped in a thin polyethylene sheet and counted with a whole-body counter. Counting generally started within 1 hr after exposure and continued on a daily schedule for 2 weeks. When possible, the dogs were counted twice daily for the first few days. After 2 weeks the

* The cooperation and assistance of the Biology Department (Inhalation Toxicology) is gratefully acknowledged. counts were made weekly and, finally, biweekly after 2 months.

The dogs were counted unanesthetized and on their backs in the center of four plutonium counter units. (34)

One unit was below the back, one above the chest, and one on each side of the chest. Since the dogs were not anesthetized, it was necessary to have someone remain in the counter and restrain them. Backgrounds were measured for all dogs and handlers before the experiment started. Counting was performed in a cave made of old steel and lined with lead.

For analysis of the counting results, two energy bands were used. One band covered the $17 \mathrm{keV} X$ ray from plutonium and the other covered the $60 \mathrm{keV} \gamma$ ray from ${ }^{241} \mathrm{Am}$.

The average empty background rate in the shield for ten background runs taken through the 2 months was $41.4 \pm 6.9$ counts/min in the X-ray region compared to $55 \pm 2.7$ counts/min in a 4 in. lead shield. (34) The background in the region of the $60 \mathrm{keV}$ gamma from ${ }^{241} \mathrm{Am}$ was $65.9 \pm 7.1$ counts/min. When a dog phantom (Alderson Research Co.) was in the counter the count rate increased to 91 and 185 counts/min for the X-ray and $\gamma$-ray regions respectively. Backgrounds for the dogs were about the same as for the phantom but were not important due to the high count rates obtained. The presence of the handler increased the background rate 
by about $20 \%$ over the empty background.

Controlling the horizontal position of the dog was a major difficulty. A line traversing the chest at the forepaws was used as reference for horizontal positioning in relation to the counter. Figure 50 shows calibration results for the phantom and the effect of small displacements on the sensitivity. Dogs do not like to be restrained on their backs and their movement was the greatest cause of errors.

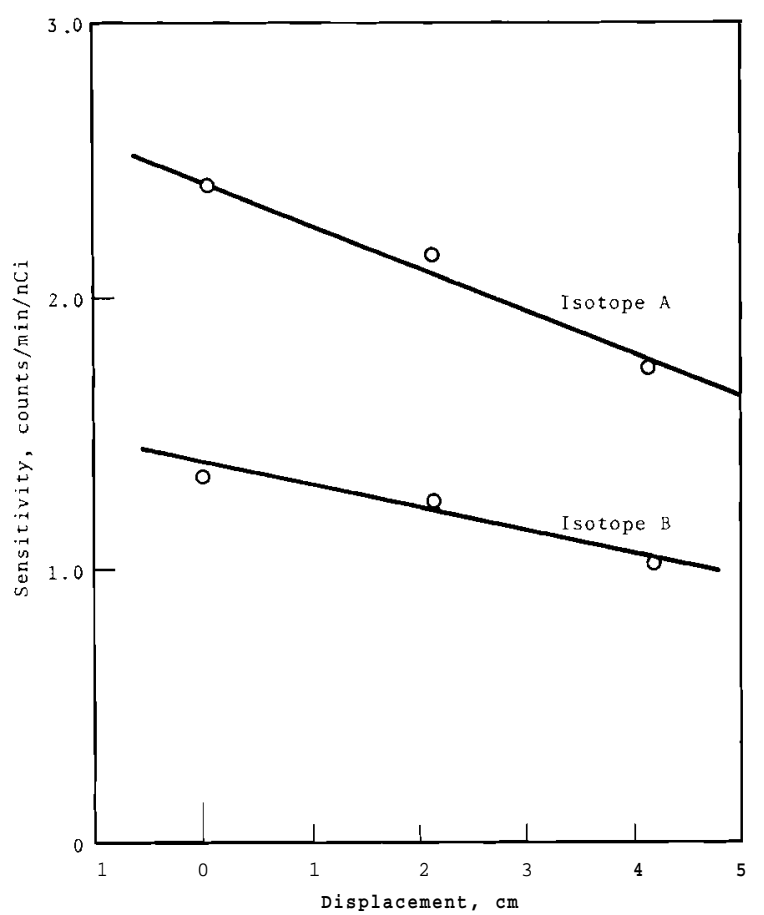

FIGURE 50. Change in Sensitivitu with Horizontal Displacement

Figure 51 shows results obtained for a typical dog. The high count rates obtained can be seen and the rapid decrease in initial count rate is obvious. An exponential elimination of the plutonium was assumed

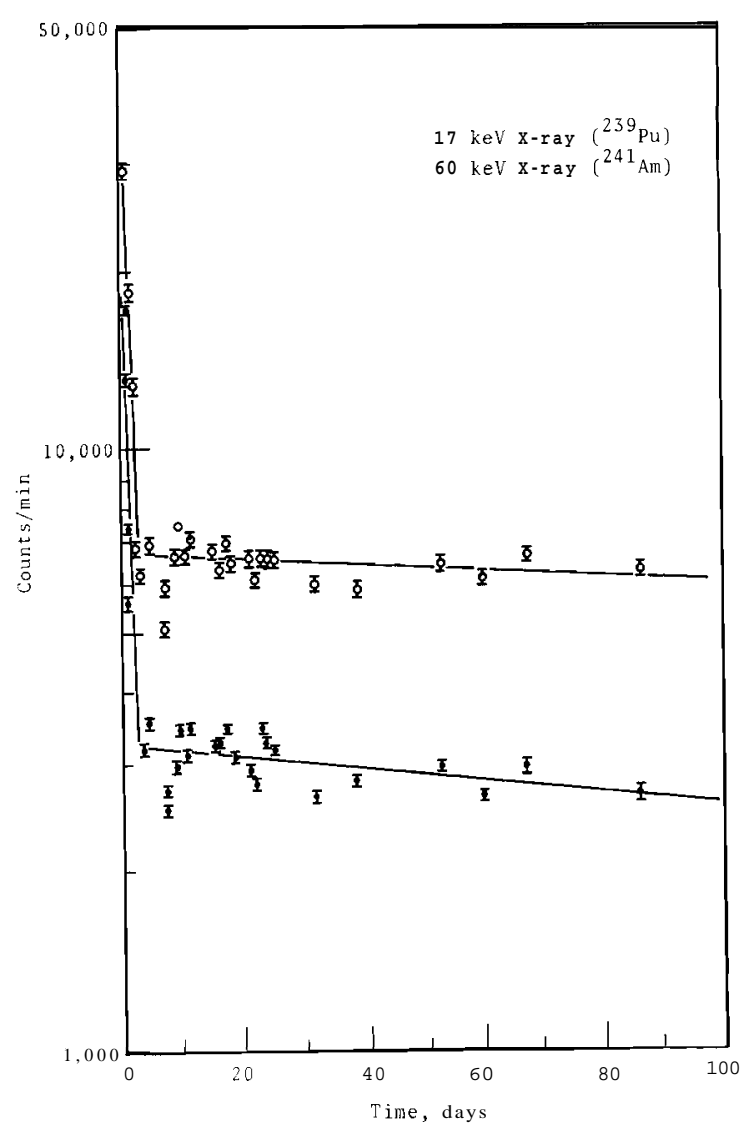

FIGURE 51. Retention of Inhaled

and the best straight line was used to fit the points. This was done for a 11 the results. A half-time for retention of the rapidly eliminated portion and the ratio of initial count rate to that when the elimination rate leveled off was calculated from each curve. Table III. summarizes the results for 12 of the dogs. One dog was not included in the analysis because he was exposed a second time to increase his body burden, but his count rates remained near background.

Only general statements can be made about the results between the 


\begin{tabular}{|c|c|c|c|c|c|c|c|c|}
\hline \multirow[t]{2}{*}{ Group } & \multirow[t]{2}{*}{ Dog } & \multicolumn{2}{|c|}{$\begin{array}{c}\text { Ratio: } \\
\text { Initial Count Rate } \\
\text { Final Count Rate } \\
\end{array}$} & \multicolumn{2}{|c|}{$\begin{array}{c}\text { Initial Retention } \\
\text { Half-Time } \\
\text { days } \\
\end{array}$} & \multicolumn{3}{|c|}{$\begin{array}{c}\text { Final Body } \\
\text { Burden, } \\
\mu \mathrm{Ci} \\
\end{array}$} \\
\hline & & $\underline{X \text { ray }}$ & $\underline{\mathrm{y} \text { ray }}$ & $\underline{\mathrm{Xray}}$ & $\underline{\text { ray }}$ & & $X$ ray & $\nvdash$ ray \\
\hline I & 306 & 5.4 & 5.25 & 0.90 & 0.85 & & 1.53 & 1.77 \\
\hline I & 311 & 2.94 & 3.79 & 0.85 & 0.86 & & 3.71 & 3.93 \\
\hline I & 313 & 2.92 & 4.10 & 1.00 & 0.95 & & 7.27 & 7.09 \\
\hline I I & 307 & 2.42 & 3.52 & 1.04 & 0.95 & & 80.7 & 62.3 \\
\hline I I & 308 & 2.8 & 3.24 & 1.41 & 1.46 & & 22.4 & 23.2 \\
\hline I I & 316 & 1.00 & 1.00 & $\infty$ & $\infty$ & & 6.47 & 6.45 \\
\hline I I & 339 & 4.74 & 5.28 & 1.16 & 1.12 & & 21.9 & 27.2 \\
\hline I I I & 317 & 2.42 & 3.10 & 0.76 & 0.79 & & 8.56 & 7.85 \\
\hline I I I & 323 & 13.5 & 13.5 & 0.31 & 0.31 & & 6.23 & 5.51 \\
\hline IV & 318 & 18.4 & 18.4 & 2.67 & 3.24 & & 1.63 & 1.49 \\
\hline IV & 334 & 10.2 & 8.03 & 0.48 & 0.69 & & 7.88 & 7.40 \\
\hline IV & 352 & 5.4 & 7.25 & 0.44 & 0.53 & & 25.4 & 23.3 \\
\hline & & $.0 \pm 5.1$ & $6.4 \pm 4.7$ & $1.00 \pm 0.62$ & $1.07 \pm$ & 0.85 & & \\
\hline
\end{tabular}

groups because of the small group sizes. The dogs exposed to the oxides made from the metal had a shorter retention time and in general seemed to lose a greater proportion of the initial burden. Only one dog retained a 11 of its initial burden.

The averages and standard deviations were calculated for the halftime of retention and the ratio of initial burden to that remaining after the initial rapid decline.

Values of $1.00 \pm 0.62$ days and $1.07 \pm 0.85$ days were obtained for the half-time of retention for the
$X$-ray and $\gamma$-ray counting results respectively. The ratios obtained for the respective results were $6.0 \pm 5.1$ and $6.4 \pm 4.7$. This shows the large variation in the percent retained and the elimination rate after inhalation exposure.

The long term retention half-time was on the order of hundreds of days. Results are not tabulated for this portion of the counting because of the large uncertainties due to the variability of the data.

Body burdens listed were determined from counts of the anesthetized dog as done in previous 
BNWL-235 2

experiments. (34) The counts were performed shortly before the dogs were sacrificed at the conclusion of the experiment. Calibration factors were taken from Figure 50 as measured with the phantom. Comparison of the results with bioassay data has not been done.

PROGRESS IN PLUTONIUM WHOLE BODY COUNTING-K. L. Swinth

Calibration was completed of the plutonium whole body counter for plutonium in the lungs 10.385 counts/min-nCi). The background component due to degradation of higher energy photons was studied and correlated with the number of high energy photons present. The errors caused by various isotopic compositions were studied. The measurements on a few subjects are described.

In vivo plutonium counting involves problems of accurate background measurement and control and correction for isotopic composition. Progress in the study of these problems is presented along with calibration results and a discussion of results from counting some subjects.

\section{Calibration}

A calibration of the plutonium counter for deposition of plutonium in the lungs, liver, and bronchial lymph nodes gave sensitivities for the respective organs of 0.385 , 0.505 , and 0.349 counts/min-nCi when the $17 \mathrm{keV} \mathrm{X}$-rays were counted. Including the $60 \mathrm{keV}$ americium gamma ray increased the respective sensitivities to 2.63, 3.60, and 2.78 counts/min-nCi. Calibrations were made for these organs because experience with animals (34) indicates that the major portion of inhaled plutonium can be expected in these organs.

The calibrations were performed with two counting units over the chest and one on each side under the arms with the top edge of the upper counters 2 in. below the top of the sternum of the reclining subject. This is referred to as the standard position. A counting unit contains 13 phototubes optically coupled to thin NaI crystals. (36) Although sensitivity for liver-deposited plutonium increases as the counter is moved lower over the body, for other organs this will not be so. Unless greater than $20 \%$ of the body burden of plutonium is in the liver, the total sensitivity will continuously decrease as the counters are moved below the standard position.

The calibrations were made using an Alderson Research Remab phantom. For the lung calibration, the plutonium was mixed in a lung equivalent material and placed in the lung cavities of the phantom. For the liver calibration a radioactive solution was used, and a small source was used for the bronchial lymph node calibration. The sources were all of the same isotopic composition.

Background Variation

Plutonium must be detected with high efficiency because of the weak 
radiations and low levels involved. A maximum permissible body burden based on bone is $40 \mathrm{nCi}$ (NBS Handbook 69) whereas one can calculate a maximum permissible lung burden of $16 \mathrm{nCi}$ based on dose to the lungs. These low limits coupled with the high absorption and low abundance of the measurable radiations makes it necessary to keep the background as low and predictable as possible.

Anticoincidence techniques (37) with a plastic phosphor have been tried; but, although this reduces the background, it is not effective in eliminating contributions from radionuclides in the subject's body. Other anticoincidence methods have been investigated with little or no improvement. This included trying a thin proportional counter over the face of one unit and using it in anticoincidence.

The major problem in background control arises from the fact that individuals have distinct and varying amounts of radionuclides in their bodies which leads to different background rates for individuals and for the same individual at various times. Differences as great as a factor of two are obtained in the background rate for unexposed individuals. The anticoincidence methods do not significantly reduce the differences in the background.

It was decided that the best approach would be to find an independent measure of the background contribution of an individual and correlate this with his background in the plutonium counter. The most desirable correlation will have an equal or greater sensitivity to changes in the background rate. Whole body counts, (37) chest scans, and simultaneous chest counts have been tried but have not proved to be any better or as simple as using a higher energy region in the plutonium counter spectrum. The region from 136 to $176 \mathrm{keV}$ is used because no significant contribution from a plutonium source was obtained in this area.

Figure 52 shows data obtained for the high energy region (136 to 176 keV) versus the background rate in the $17 \mathrm{keV}$ X-ray channels. Figure 53 shows the correlation for the channels including the $X$ ray plus the $60 \mathrm{keV}$ 241 Am gamma ray. Also shown on both

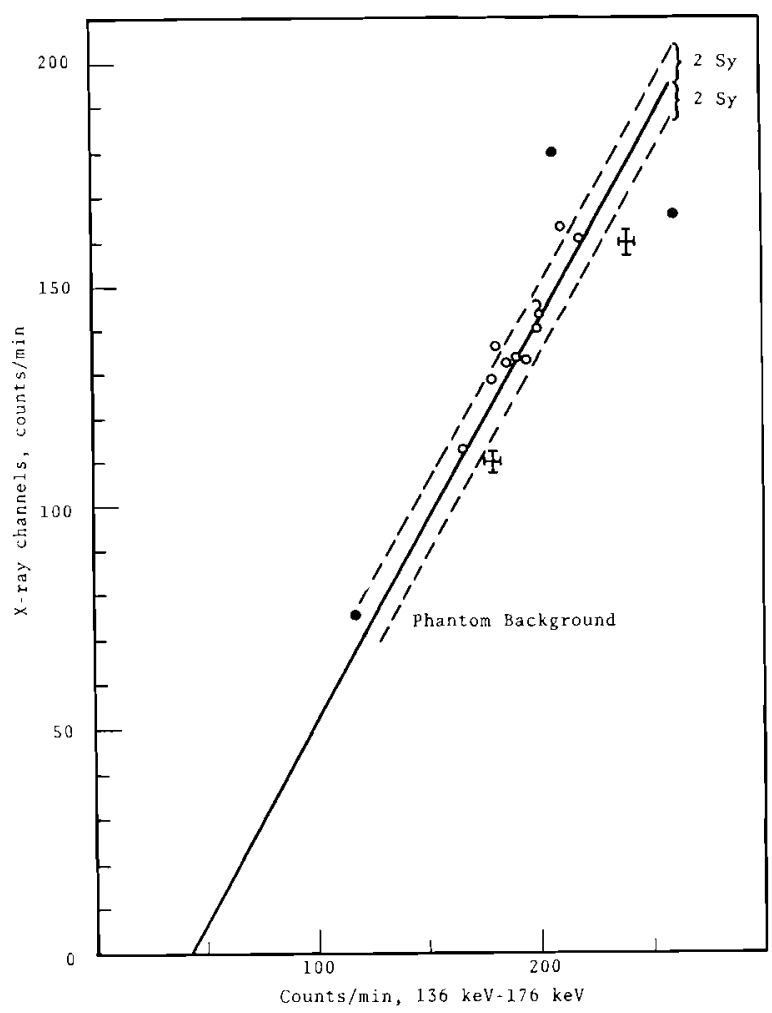

FIGURE 52. Count Rate of Unexposed People (17 keV X Ray) 


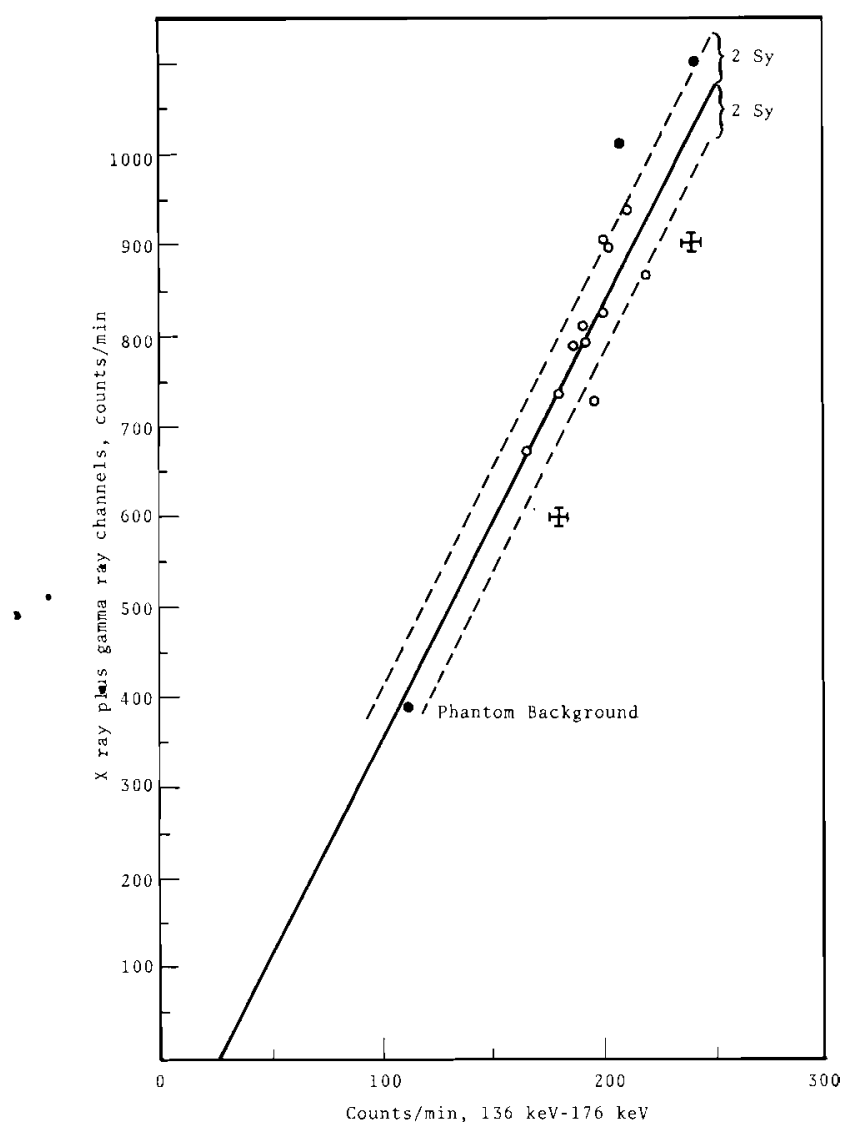

FIGURE 53. Count Rate of Unexposed People 117 keV X Ray Plus 60 keV $\gamma$ Ray)

figures is a least squares fit to the points which is bordered by two dotted lines at twice the standard error of estimate. The solid circles are not included in the least squares analysis. Correlation coefficients were calculated for both cases and indicated that the correlation was highly significant. The least squares fit for the $X$ ray channels is $y=0.92 x-39.7$; when the ${ }^{241} \mathrm{Am}$ gamma is included it is $\mathrm{y}=4.80 \mathrm{x}-123$. The standard errors of estimate are 3.8 and 29.6 counts/min for the respective cases. Including the solid circles for the $X$ rays changes the least squares equation to $y=0.82 x-19.5$ and the standard error of estimate increases to 9.5 counts/min. These two points will be ignored in the subsequent analysis since they are attributable to the poor counter geometry resulting from the sizes and shapes of the individuals.

It is desirable to state a lower limit of detection for the counter. If we assume that the data points are normally distributed about the least squares line and use the standard error of estimate rather than the square root of background-rate as a measure of the standard deviation, one can estimate the limits of detection.

From the work of Altshuler and Pasternack ${ }^{(35)}$ one calculates for the "minimum significant measurable activity," 19 nCi for the X-ray channels and $22 \mathrm{nCi}$ for the X-ray plus ${ }^{241}$ Am gamma-ray channels. These correspond to two standard deviations of the background. Only plutonium deposited in the lungs is considered here. The "minimum detectable true activity" is 49 nCi for the X-ray region and $46 \mathrm{nCi}$ when the higher energy region is included. In the calculations the probabilities of judging true activity as background and true background as activity have been set at $2.5 \%$.

The $1 \mathrm{imits}$ of detection do vary with distribution of the isotope and isotopic composition. These features are discussed elsewhere in this report.

Measurements on Subjects

The counter has had limited use in evaluating internal exposure to plutonium. Personnel exposed in glove 
box incidents at Rocky Flats and Hanford were counted.

Two subjects from Rocky Flats were counted; only one of these cases will be described. A complete description of the accident and treatment was given by Putzier et al. (38) At the time of the counts, background estimation by correlation with count rate from 136 to $176 \mathrm{keV}$ in the plutonium counter was not being performed. The only data available to estimate the individual's background rate were his whole body counts.

The data from the X-ray region indicated a body burden of $520 \mathrm{nCi}$ while a body burden of $410 \mathrm{nCi}$ was indicated when the ${ }^{241}$ Am gamma ray was included. Putzier's data give a body burden of $610 \mathrm{nCi}$ about $21 / 2$ month previous to our counts. It should be noted that a correction for isotopic composition was not made and could account for the difference. A correction was made for distribution. The distribution was measured by using one counter unit over the individual lungs and liver. The selfcollimating property of low energy radiation was assumed, and a distribution similar to that assumed by Putzier was obtained. In that work the activity ratio (right lung to left lung to liver) was $1: 1.3: 1.1$ while in the present case it was $1: 1.4: 1$.

Eleven people at Hanford were involved in a glove box incident with possible exposure to an aerosol of uranium enriched with plutonium. All of these individuals were screened for possible lung burdens. The results were negative for all but two individuals, and one of these was at background levels one day later.

The positive individual had a count rate indicating a possible body burden of $250 \mathrm{nCi} 24 \mathrm{hr}$ after the incident. The activity disappeared rapidly with half of it gone in $38 \mathrm{hr}$ indicating removal by ciliary action. Counting results indicated that the individual reached background levels 5 to 6 days after the incident.

Bioassay results indicated a retained body burden below the limits of detection of the counter. Correction was not made for isotopic composition of the exposure material.

\section{Isotopic Composition}

In the section on calibration it was noted that the same isotope was used in all of the cases. This is significant since the $X$-ray emission rate changes with the isotopic composition. Calculations were made of this effect. Figure 54 shows the calculated number of $17 \mathrm{keV} \mathrm{X}$-rays emitted per alpha emitted for four different isotopic compositions and how this number changes with time. For dosimetry purposes, it is the $\mathrm{X}$-ray/alpha ratio that is of interest, but it must be remembered that the alpha emission rate increases with time, so the change in total X-ray emission with time is greater than indicated by the figure.

Due to the large differences in $X$-ray/alpha ratios possible, it is desirable to know this ratio when interpreting whole body ounting resuits. A summer student worked on

\footnotetext{
* J. Schultz, AEC Health Physics Fe Zlow, University of Washington
} 


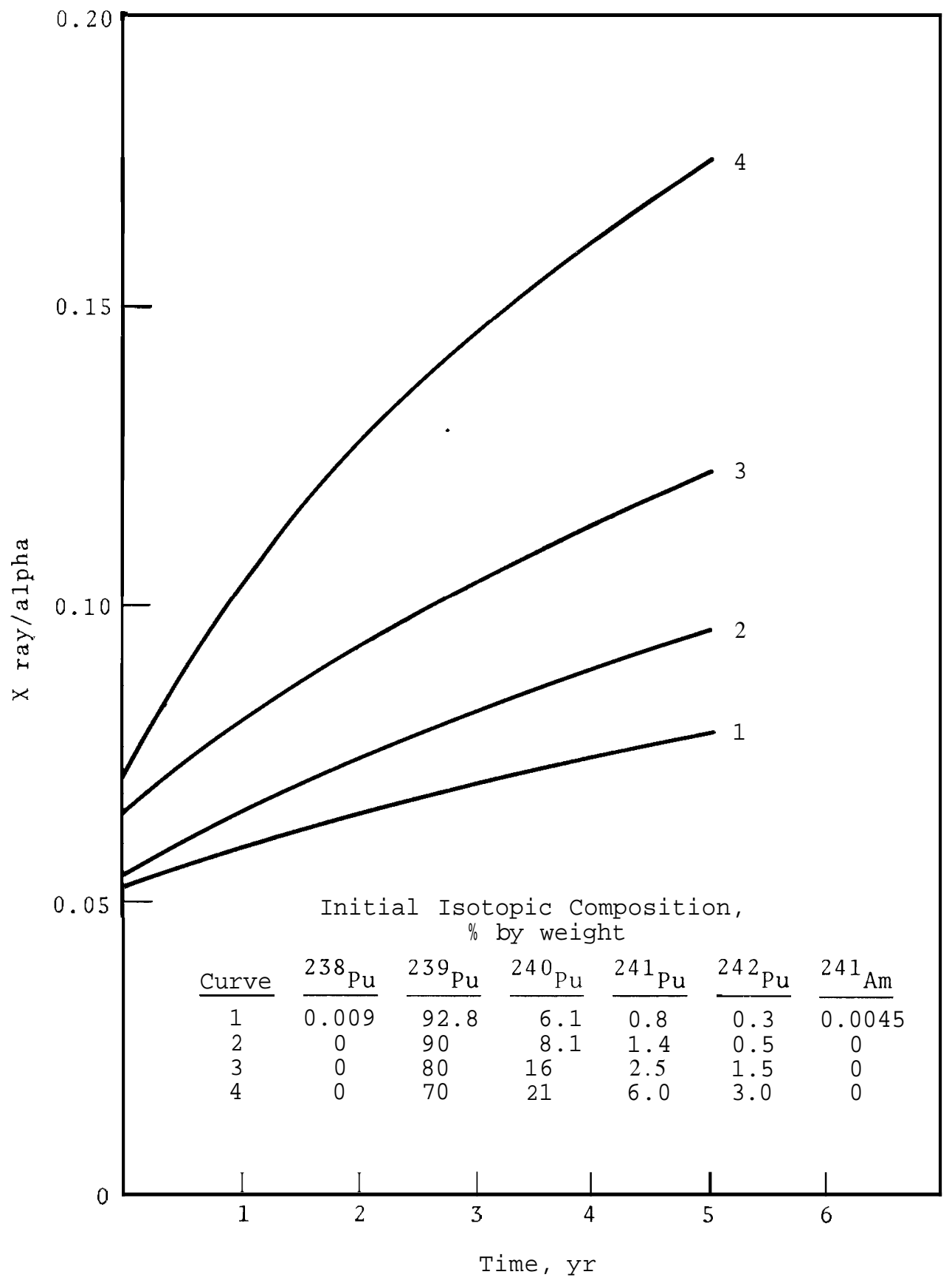

FIGURE 54. Secular Increase in X Ray per Alpha Emitted by Several Isotopic Mixtures of Plutonium

the assembly and evaluation of a counter for the determination of this ratio. The counter is a gas flow proportional counter containing the source and designed to detect the alpha particles.
A thin NaI crystal makes up one wall of the counter and detects the $\mathrm{X}$ rays associated with the decay of the plutonium. Figure 55 shows the general scheme of the detection system. 


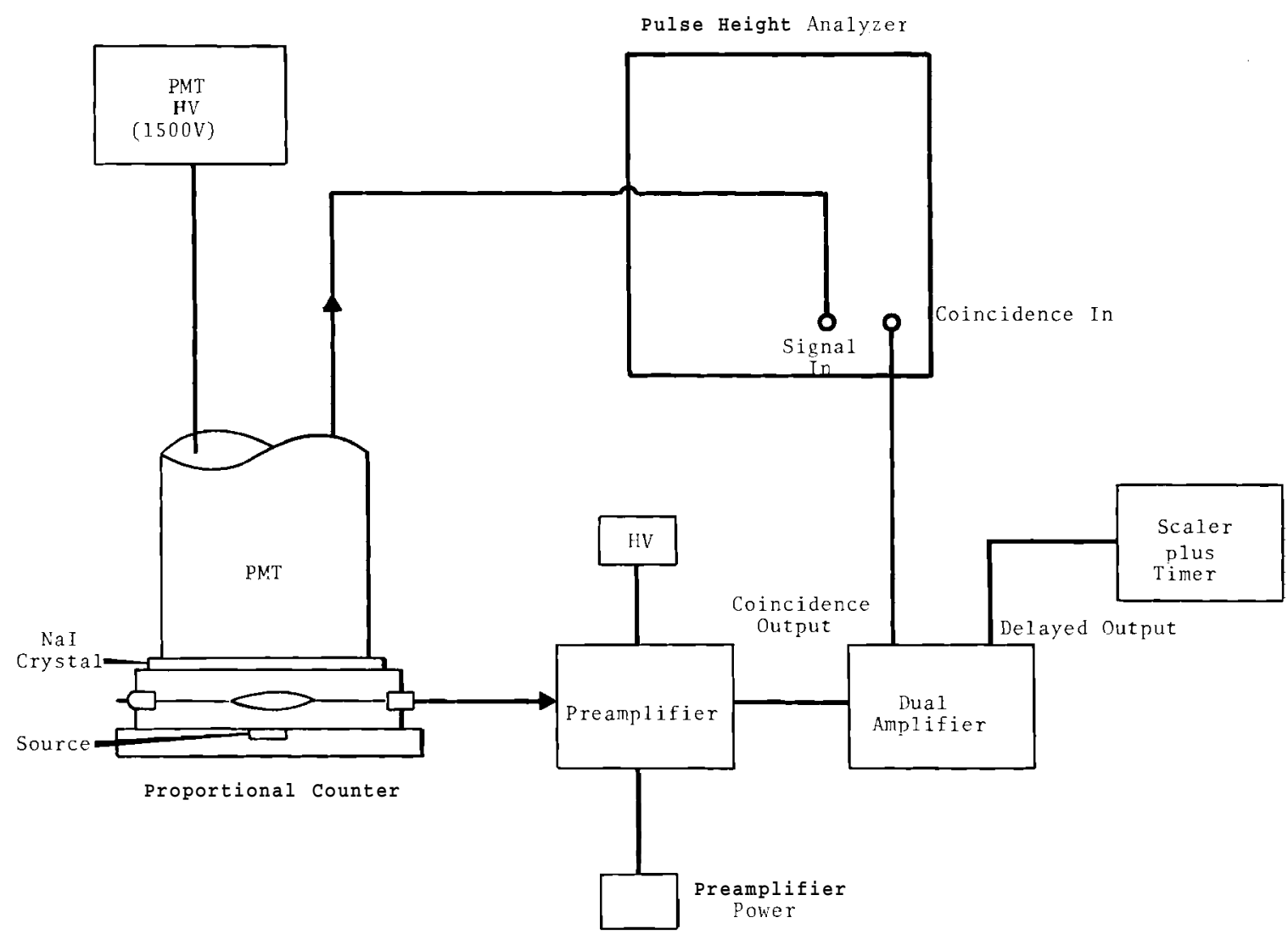

FIGURE 55. Schematic Diagram of X Ray $\overline{t o}$ Alpha Ratio Experiment

${ }^{55} \mathrm{Fe}$ IN THE MARINE ENVIRONMENT AND IN PEOPLE WHO CONSUME OCEAN FISHH. E. Palmer and T. M. Beasley

A concentration process for ${ }^{55}$ Fe exists in the marine environment which far exceeds the concentration that occurs in the lichen-caribou-Eskimo food chain. In salmon and northern tuna this process leads to amounts of Fe that are 20 to 30 times the amount in the Alaskan caribou muscle and as much as 100 times those in United States beef muscle. Body burdens of $55 \mathrm{Fe}$ approaching $1.5 \mu \mathrm{Ci}$ have been measured in people who eat large amounts of salmon.

The levels of ${ }^{55} \mathrm{Fe}$ in humans and their food both in Alaska and in the State of Washington were recently reported. During this investigation, it was found that ocean fish had higher concentrations of this radio- 
nuclide than did the Alaskan caribou. This finding suggested that individuals whose diet contained large quantities of ocean fish could have body burdens several times higher than those of the caribou-eating Eskimos and perhaps several orders of magnitude higher than those of the Richland, Washington, residents measured. This report details the measurements that have been made on samples from the marine environment and includes the estimated ${ }^{55} \mathrm{Fe}$ body burdens of individuals who derive a major portion of their diet from ocean fish.

${ }^{55} \mathrm{Fe}$ concentrations in ocean fish have been reported previously; $(39,40)$ however, the measurements were made before the last series of atmospheric nuclear tests. The levels in salmon and tuna fish are now 100 to 200 times the levels reported for these earlier measurements. We have analyzed a number of marine samples in an attempt to indicate the ${ }^{55} \mathrm{Fe}$ concentrations in the various trophic levels of the marine environment and to point out the relative concentrations of ${ }^{55} \mathrm{Fe}$ in those marine organisms that serve as foodstuffs for human beings.

Table IV contains the result of the analysis of a variety of ocean fish using the methods previously described. (41) Several interesting points are evident from these data. First, it is seen that salmon, tuna, and saury fish have the highest

${ }^{55}$ Fe in Ocean Fish During Last 6 Months of 1965

\begin{tabular}{|c|c|c|}
\hline \multicolumn{3}{|c|}{ Number of Samples } \\
\hline (F1esh) & & \\
\hline Salmon & 1 & Ocean at Kotzebue, Alaska \\
\hline Salmon & 1 & Ocean a t Kotzebue, Alaska \\
\hline Salmon & 3 & Yukon River in Alaska \\
\hline Salmon & 1 & Cook Inlet, Alaska \\
\hline Salmon & 1 & Washington Coast \\
\hline Albacore Tuna & 1 & Northern Pacific Ocean \\
\hline Albacore Tuna & 1 & Southern Pacific Ocean \\
\hline Albacore Tuna & 1 & Northern Atlantic \\
\hline Albacore Tuna & 1 & Southern Atlantic \\
\hline Ocean Perch & 1 & Pacific \\
\hline Cod & 1 & Pacific \\
\hline Whitefish & 3 & Northern Alaskan Coast \\
\hline Shee Fish & 2 & Northwestern Alaskan Coast \\
\hline Sea Bass & 1 & Washington Coast \\
\hline Swordfish & 1 & Pacific \\
\hline Herring & 2 & Packed in Norway \\
\hline Herring & 3 & Canadian West Coast \\
\hline Sardines & 2 & Packed in Norway \\
\hline Sardines & 2 & Packed in Maine \\
\hline Sme 1t & 2 & Washington Coast \\
\hline Saury & Several & Oregon Coast \\
\hline
\end{tabular}

\begin{tabular}{|c|c|}
\hline $\begin{array}{c}\text { Average } \\
\text { Stable Iron } \\
\text { Content, } \\
\end{array}$ & $\begin{array}{c}\text { Average } \\
55 \text { Fe Content } \\
{ }^{5} \text {. }\end{array}$ \\
\hline $\mathrm{mg} / \mathrm{kg}$ & $\mathrm{nCi} / \mathrm{kg}$ \\
\hline 12.0 & 93.0 \\
\hline 4.6 & 48.0 \\
\hline 13.2 & 37.0 \\
\hline 14.2 & 34.0 \\
\hline 5.8 & 14.0 \\
\hline 19.1 & 42.0 \\
\hline 16.3 & 1.6 \\
\hline 11.1 & 8.4 \\
\hline 4.5 & 0.78 \\
\hline 1.9 & 0.85 \\
\hline -. & 0.20 \\
\hline 11.0 & 0.10 \\
\hline - - & 0.48 \\
\hline 63.7 & 0.27 \\
\hline-- & 0.33 \\
\hline - - & 20.0 \\
\hline 11.2 & 0.79 \\
\hline -- & 15.0 \\
\hline - - & 2.6 \\
\hline 18.2 & 0.41 \\
\hline 52.3 & 106.0 \\
\hline
\end{tabular}


content of ${ }^{55} \mathrm{Fe}$. Saury fish is processed in Japan and sold for human consumption but only in a very small amount compared to the amount of salmon and tuna. The saury fish that we analyzed were taken in the plume of the Columbia River outlet, and some of the ${ }^{55} \mathrm{Fe}$ in these fish probably comes from the spent cooling water of the Hanford nuclear reactors which empties into the river. More studies are needed to determine the fraction of ${ }^{55} \mathrm{Fe}$, from the Columbia River, in these saury fish. The concentrations of ${ }^{55} \mathrm{Fe}$ in herring and sardines canned in Norway are relatively high at about $15 \mathrm{nCi} / \mathrm{kg}$; whereas, the same type of fish from the U.S. and Canada are one tenth of that. We have not measured enough samples to determine if this difference is consistent or why the difference exists. Secondly, tuna caught in the northern oceans have more ${ }^{55} \mathrm{Fe}$ than those caught in the southern oceans, and those in the Pacific Ocean have more ${ }^{55} \mathrm{Fe}$ than those in the Atlantic Ocean. These differences in ${ }^{55} \mathrm{Fe}$ concentrations of ocean fish from different geological locations are consistent with those found in measuring ${ }^{137} \mathrm{Cs}$, (42) ${ }^{60} \mathrm{Co}$, and ${ }^{110} \mathrm{Ag}$; ${ }^{(43)}$ however, the interesting observation is that the ${ }^{55}$ Fe concentrations are approximately 1000 times higher than the reported values of the other three is otopes.
The relatively high levels of ${ }^{55} \mathrm{Fe}$ in the marine species measured is due in large part to the low concentration of stable iron in sea water

( $\sim$ 1 $\quad$ g 1 i t e Also most of these marine species are iron deficient compared to land animals. Furthermore, radioactive iron falling on land surfaces will be diluted by the large amount of stable iron existing there; and, as a result, the uptake of ${ }^{55} \mathrm{Fe}$ by plants and animals will be greatly reduced because of this dilution. Salmon and tuna fish, however, are fast growing fish and, living in a low iron environment, probably take up and retain most of the iron available to them. The slower-growing fish such as sea bass, perch, and whitefish contain much less ${ }^{55} \mathrm{Fe}$. The lower ${ }^{55} \mathrm{Fe}$ to stable iron ratio which was measured in these fish confirms the lower uptake and slower growth.

Table $V$ contains data showing the relative concentrations of ${ }^{55} \mathrm{Fe}$ in the liver, roe, and muscle of two chum salmon caught at Kotzebue, Alaska. The livers of these salmon contain almost $2 \mu \mathrm{Ci}$ of ${ }^{55} \mathrm{Fe}$ per kilogram of liver (wet weight) and represent the highest concentrations we have measured in any organism or tissue. It is also interesting to note the rather uniform labelling of the salmon as evidence by the ratio of radioactive to stable iron

TABLEV. ${ }^{55}$ Fe ana Stable Iron in Salmon Caught at Kotzebue, Alaska

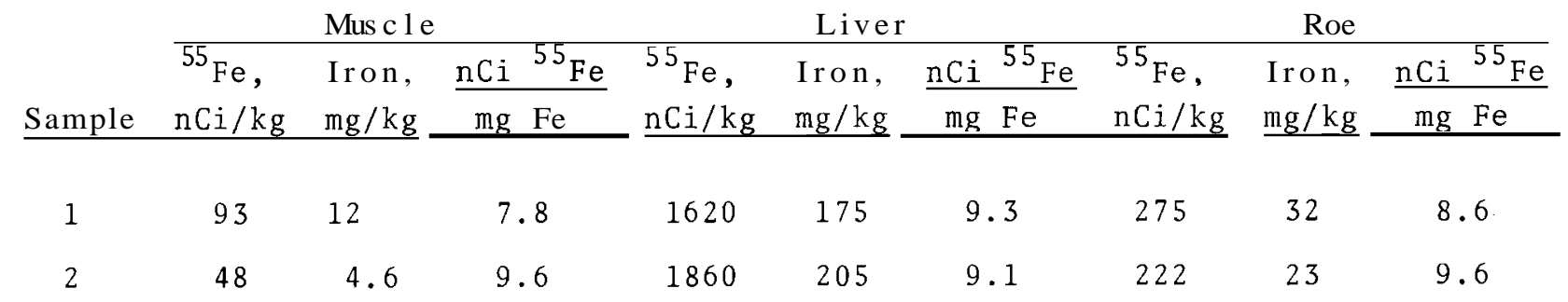


in the various tissues.

Since salmon are known to feed on a variety of marine orgaisms (plankton, euphausids, herring, squid, etc.) it is difficult to attribute their high ${ }^{55} \mathrm{Fe}$ concentrations to the consumption of a specific species. Indeed, their high levels probably result from the intake of both the relatively high activity of small fish such as herring or saury and the lower activity plankton, euphausids, and squid. Table VI lists some representative values of the ${ }^{55} \mathrm{Fe}$ concentrations in these tropic levels. The barnacles are surface-living organisms that were taken from glass floats off the California coast, and their high ${ }^{55} \mathrm{Fe}$ content indicates a large uptake from fresh fallout on the ocean surface. The higher availability of the ${ }^{55} \mathrm{Fe}$ at the surface may be the reason for the high levels in saury fish since they also live close to the surface. The sea cucumbers are mud scavengers and these samples were taken from the Columbia River plume which probably accounts for their high ${ }^{55} \mathrm{Fe}$ content. Again additional studies are needed to determine the fraction of ${ }^{55} \mathrm{Fe}$ coming fram the Columbia River. other marine organisms such as oysters, scallops, clams, shrimp, and crab are very low in ${ }^{55} \mathrm{Fe}^{(41)}$ and probably do not contribute much to the ${ }^{55} \mathrm{Fe}$ body burdens of either other marine species or humans.

As might be expected, these high $1 \mathrm{ev}-$ els of ${ }^{55} \mathrm{Fe}$ in the salmon produce higher than average levels of ${ }^{55} \mathrm{Fe}$ in populations that eat substantial quantities of these fish. As was reported, (41) the caribou-eating Eskimo of Anaktuvuk, Alaska had an average ${ }^{55} \mathrm{Fe}$ body burden of $61 \mathrm{nCi}$ in January, 1965. In November, 1965, this same group of people had an average ${ }^{55} \mathrm{Fe}$ body burden of 81 nCi, an increase of some $13 \%$. In contrast, adult male Eskimos from Tanana, Alaska, who eat large quantities of sal-

TABLE VI. Concentration of ${ }^{55}$ Fe in some Marine organisms

Species

Sea Water

Phytoplankton (dinoflagellata)

Euphausids

Mussel (Mytilus)

Kelp (Nacrystis)

Barnacles (Lepas)

Squid Muscle (Lolego)

Squid Liver

Purple Sea Cucumbers (whole)

Sea Urchins (whole)
Location

$\mathrm{nCi} / \mathrm{kg}$

California Coast

$<0.00004$

California Coast

0.5

Oregon Coast

1.5

Gulf of Alaska

0.16

California Coast

0.36

California Coast

0.03

California Coast

140.0

California Coast

0.76

California Coast

8.6

Oregon Coast

76.0

Oregon Coast
0.48 
mon in addition to caribou and moose meat, showed an average ${ }^{55} \mathrm{Fe}$ body burden of $252 \mathrm{nCi}$. Furthermore, recent blood samples received from natives near Bethel, Alaska, who eat more salmon than the Tanana natives show that their average ${ }^{55}$ Fe body burden is 700 nCi with one individual having a body burden of $1440 \mathrm{nCi}$.

In the analysis of the blood specimens obtained from the Bethel natives, it was found that the average ${ }^{55} \mathrm{Fe}$ body burden of the females was twice that of the males. The opposite is true for ${ }^{137} \mathrm{Cs}$ in the Alaskan natives where the content in women is consistently two thirds that in the males (44) due to their lower dietary intake. Since the food intake of women is generally lower than that of men, the higher ${ }^{55} \mathrm{Fe}$ body burdens observed probably reflect the more rapid turnover of iron by females. This is probably due to the iron loss through the menstrual blood with the result that their body burdens are closer to an equilibrium with the ${ }^{55} \mathrm{Fe}$ in their diet than are the males.

Despite the fact that some of the Alaskan natives have body burdens approaching $1.5 \mu \mathrm{Ci}$, which is about 100 times the average level of residents of the State of Washington, it must be emphasized that these levels are far below the maximum permissible body burden of $100 \mu \mathrm{Ci}$ for members of the general population who are not exposed to ${ }^{55} \mathrm{Fe}$ in the course of their occupations. (45) The radiation dose to the red blood cells of a person with a constant ${ }^{55} \mathrm{Fe}$ body burden of $1000 \mathrm{nCi}$ would be about $30 \mathrm{mrad} / \mathrm{yr}$. This estimate is calculated using both the X-ray and Auger electron dose to the erythrocyte fraction of the blood. This dose is about $30 \%$ of the yearly dose to the body from natural sources such as cosmic rays and internally deposited ${ }^{40} \mathrm{~K}$ and radium.

As a result of these investigations one might predict that populations in the northern countries such as Japan and the Scandinavian countries who eat large quantities of ocean fish probably have relatively high ${ }^{55} \mathrm{Fe}$ body burdens as do the Alaskan natives. In our present investigations, we are measuring the ${ }^{55} \mathrm{Fe}$ content in various populations of the world.

We thank R. M. Bernard and T. A. Jokela for their assistance in analyzing the many samples, Dr. J. M. Aase and Dr. H. D. Miller of the U.S. Public Health Service in Alaska for obtaining blood samples from Alaskan natives, and Mr. Max Brewer, Director of the Arctic Research Laboratory, for the support in obtaining the many Alaskan samples.

SEASONAL CYCLE OF ${ }^{137} \mathrm{CS}$ I N ALASKAN NATIVES AT ANAKTUVUK PASS - H. E. Palmer W. C. Hanson* and L. A. Braby

The seasonal cycle of ${ }^{137} \mathrm{Cs}$ in the Anaktuvuk Pass natives which was started in January, 1964, was continued until April, 1965. Maximum

*Biology Department burdens occurred during spring and summer months and then decreased steadily until the following spring. This seasonal cycle is primarily dependent upon the availability of caribou which forms the food base 
for the village and which showed a different seasonal cycle of ${ }^{137} \mathrm{Cs}$ in the meat. Figure 56 shows the seasonal variation of the body burden in the adult Eskimos from January, 1964, to April, 1965. The details of this study are published elsewhere. (46)

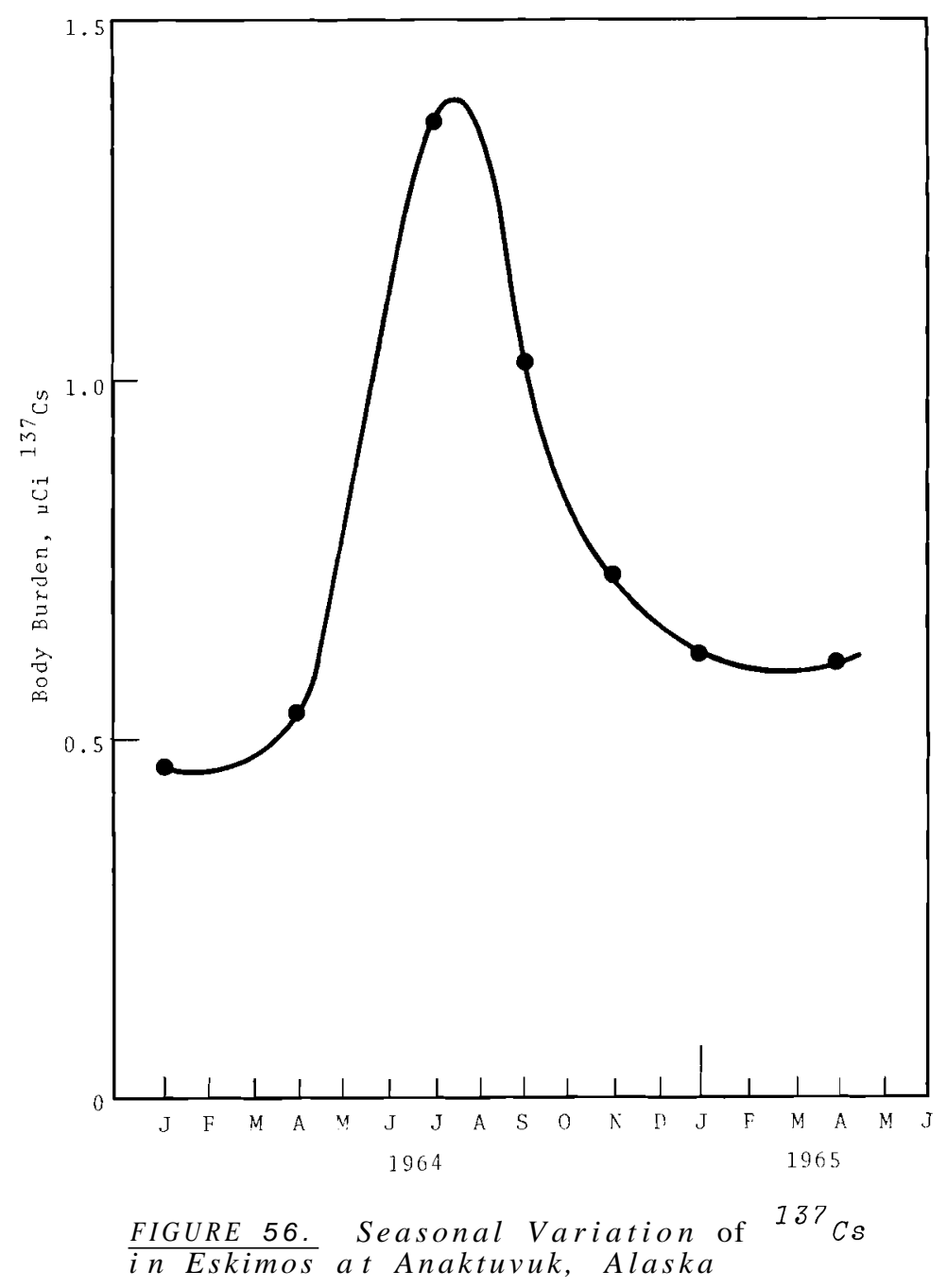

THE APPLICATION OF SIMPLIFIED WHOLE BODY COUNTING IN ALASKA-H. E. PaImer

The development of the simplified whole body counting method, which uses a 3 by 3 in. NaI crystal in conjunction with a battery-powered, transistorized, single channel analyzer with a built-in high voltage supply, was described previously. $(47,48)$
The crystal, instrumentation, and scales for weighing the people can be carried in a medium-sized suitcase.

This method was successfully used in the past year to measure the body burdens of adults at the remote villages of Ambler, Arctic Village, 
and Anaktuvuk Pass in Alaska. The average ${ }^{137} \mathrm{Cs}$ burdens of adult males in April, 1964, were 834, 1218, 734 $\mathrm{nCi}$, respectively. With this simplified method we were able to reach a village, count about 20 people, and return to a central point within a few hours. Our past methods would have taken about a week at each village (plus much higher transportation costs) to obtain the same information.

Because of the convenience of this method, it has entirely replaced the use of the shadow shield whole body counter in the studies of ${ }^{137} \mathrm{Cs}$ in Alaskan natives. 


\section{RADIATION CHEMISTRY}

\section{DETERMINATION OF ERYTHROCYTE SIZE DISTRIBUTION-W. D. Felix}

Electronic instruments are now commonly used to count particle concentrations in electrically conducting suspensions. The Coulter Counter counts particles by sensing the changes in resistivity between two conduction liquid bodies connected by a small orifice.

The volume of a particle is related to the change in resistance detected across the orifice as a particle passes from one chamber to another through the orifice. By using a pulse-height analyzer, the volume distribution of particles flowing through the orifice can be automatically stored and recorded.

Many variables, which may have negligible effect on the determination of absolute numbers of cells present in a saline suspension, significantly affect the determination of volume distribution. Reported here are the observations of the effects of electronic resolution, pH, counting rate, and dilution time. The results were used to establish standard procedures for volume distribution studies in this laboratory.

\section{Experimental}

Erythrocytes were diluted in normal, buffered saline (phosphate buffer, $\mathrm{pH}$ 7.4). Signals generated by cells passing through the orifice were recorded on a 256-channel analyzer. Aperture current setting on the Model $B$ Coulter Counter was 0.354 corresponding to a nominal current of $1.41 \mathrm{~mA}$; gain,
$1 / A$, was $1 / 2$. Unless otherwise noted, 100,000 cells were counted in $90 \mathrm{sec}$ corresponding to a dead time of less than $10 \%$ on the 256-channel analyzer.

\section{Aperture Effects}

Resolution of the distribution spectrum can be improved by prolonging the time the instrument has to sense the electrical signal resulting from a particle passing through the orifice. This increase in time for sensing may be gained by decreasing the rate through the orifice, by increasing the effective measurement volume by elongation of the aperture, or by increasing the critical volume of the electrical field around the aperture by the use of large aperture currents. Lushbaugh et al.(49) investigated the latter effect and found an apparent increase in resolution. Their further findings were considered as biological evidence for the existence of two distinct subpopulations of erythrocytes in mammalian blood.

This subpopulation due to "mature" cells as opposed to "immature"cells was manifest in electronic measurements as a shoulder in the size distribution curve. (50)

Kubitschek(51), in examining the distribution analysis of erythrocytes, approached the problem by using elongated apertures whose lengths were two or three times the diameter. Resolution was increased by about $20 \%$ and the coefficient of variation was decreased by about $50 \%$. 
Several aperture tubes were constructed from precision bore, $100 \mu$ diam capillary tubing, sliced into wafers 200 to $300 \mu$ thick and cemented over an opening in a tube similar in dimension to that supplied with the Coulter Counter.

The distribution of human erythrocytes obtained from the elongated aperture is compared with that obtained from the commercial aperture in Figure 57. It was necessary to use a larger current across the elongated aperture than across the short aperture since the effective field was much larger. It is readily obvious that the shoulder which had been present in the distribution from the commercial aperture tube is not present in the distribution curve from the elongated aperture tube.
In fact, the latter curve is nearly symmetrical. This finding is in contradiction to the proposal of Lushbaugh, et al., (50) of a two component erythrocyte system.

\section{Counting Rate Effect}

Increasing the counting rate beyond the optimum rate results in a shift of the distribution peak to larger volumes due to coincident passage of cells. Decreasing the cells per unit volume results in inconveniently long counting times. A convenient indicator during operation for the number of cells counted per unit time is given by the percent dead time on the analog to digital conversion unit. Two curves

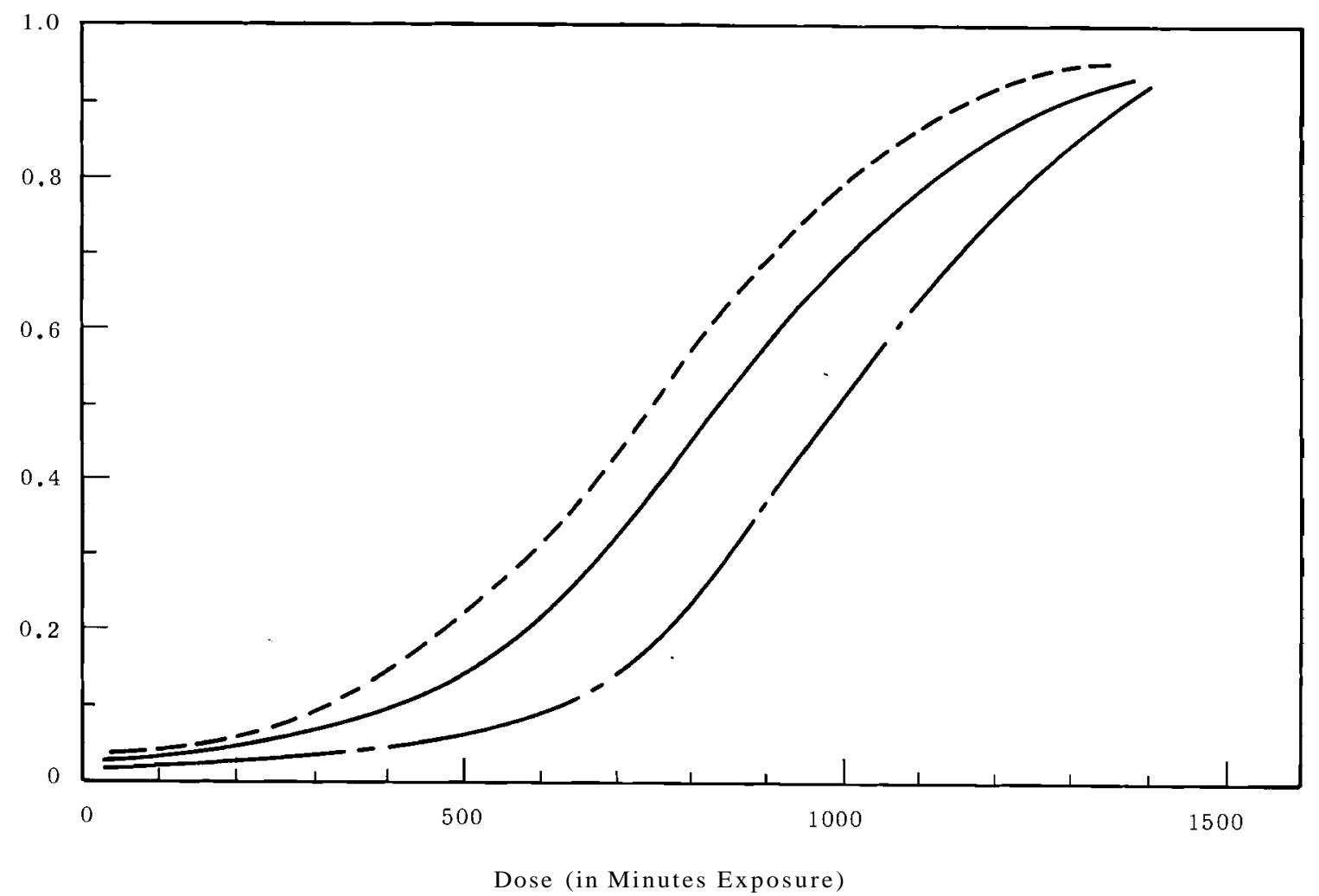

FIGURE 57. Comparison of CeZl Distributions Obtained from Normal and EZongated Apertures 
resulting from counts made at two different dead times are shown in Figure 58. The distribution given by the $11 \%$ dead time count has been noticeably shifted to a larger volume. Our findings with a 256-channel analyzer indicate that distributions made with $10 \%$ dead times, or less, give reproducible peak volumes. Using $200 \mu$ aperture tubes and under the pressure differential obtained with the "fish tank" vacuum pump supplied with the Coulter Counter, a $10 \%$ dead time corresponded to a live counting time for 100,000 cells of approximately $90 \mathrm{sec}$.

$\underline{\text { Dilution Time and } \mathrm{pH} \text { Effects }}$

For procedures using the Coulter Counter, one normally attempts to save time by diluting all of the samples to

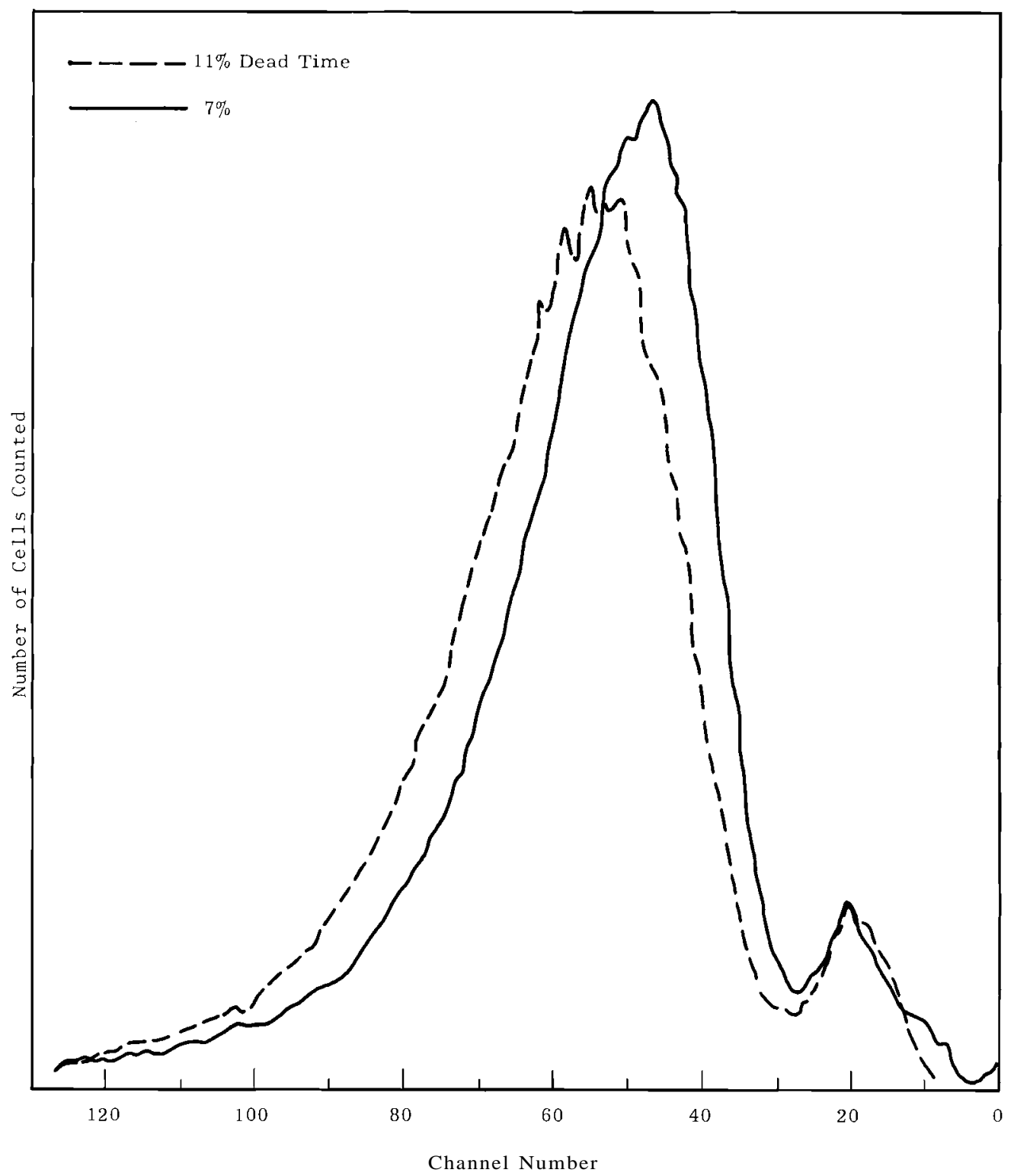

FIGURE 58. Effect of Counting Rate on CelZ Size Distribution 
be counted at once. The question arises whether erythrocytes undergo enough volume change during the time between dilution and counting to affect the sizing of the cells. Concentrated cell suspensions were diluted in normal, filtered saline $(0.9 \% \mathrm{NaCl})$ and in normal, buffered saline (phosphate buffer, pH 7.4). Figures 59 and 60 show the size distributions made at times up to 26 min following initial dilution. The 26 min sizing in buffered solution was not significantly different from that at 18 min and is consequently not shown.

One must conclude that distribution counts must be taken immediately on dilution. In fact, during 90 sec counts, there will undoubtedly be some broadening of the distribution curves due to

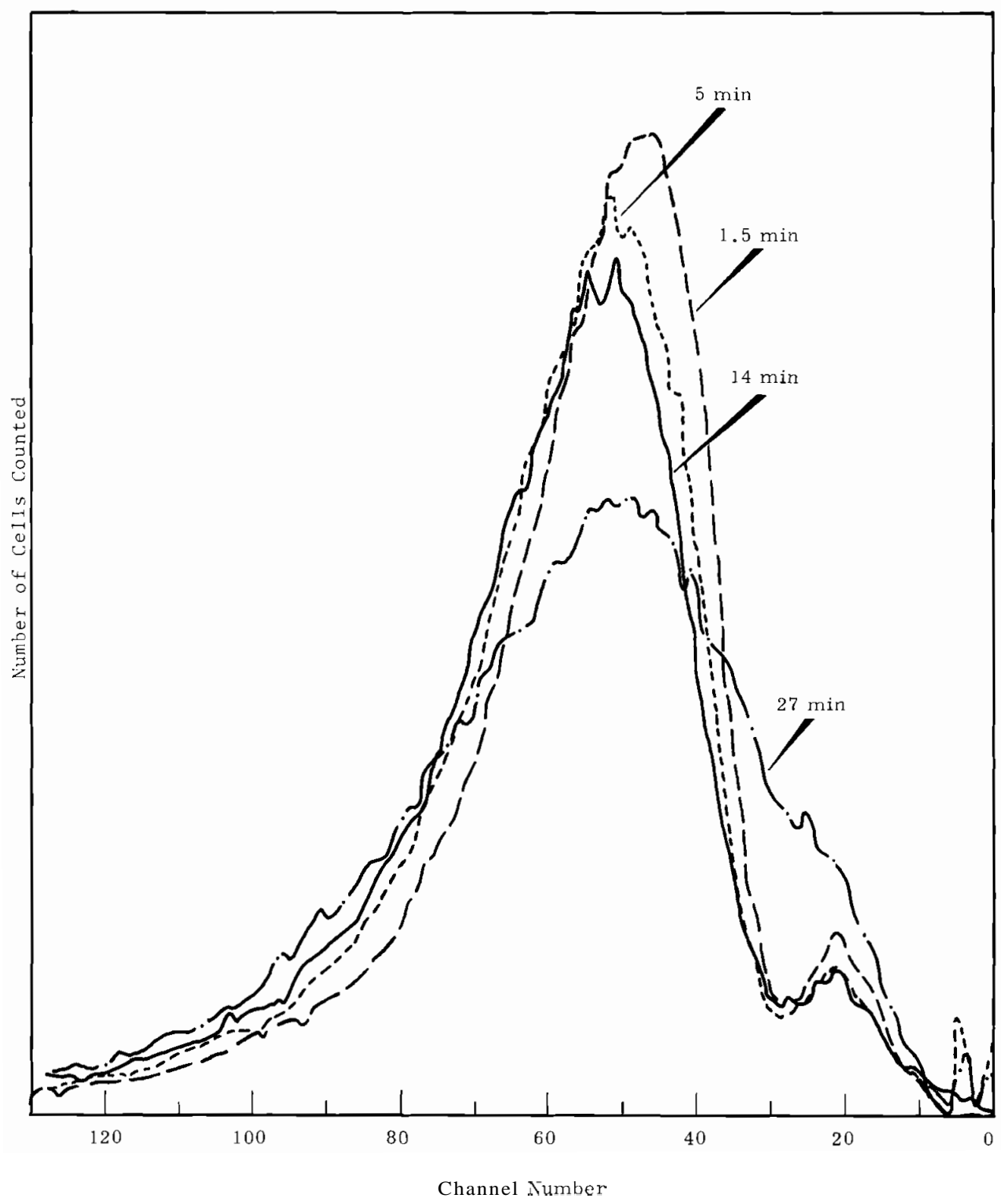

FIGURE 59. Effect of DeZay Between Dizu-

tion and Counting on CeZZ Size Distri-

bution Determination. Normal Saline 


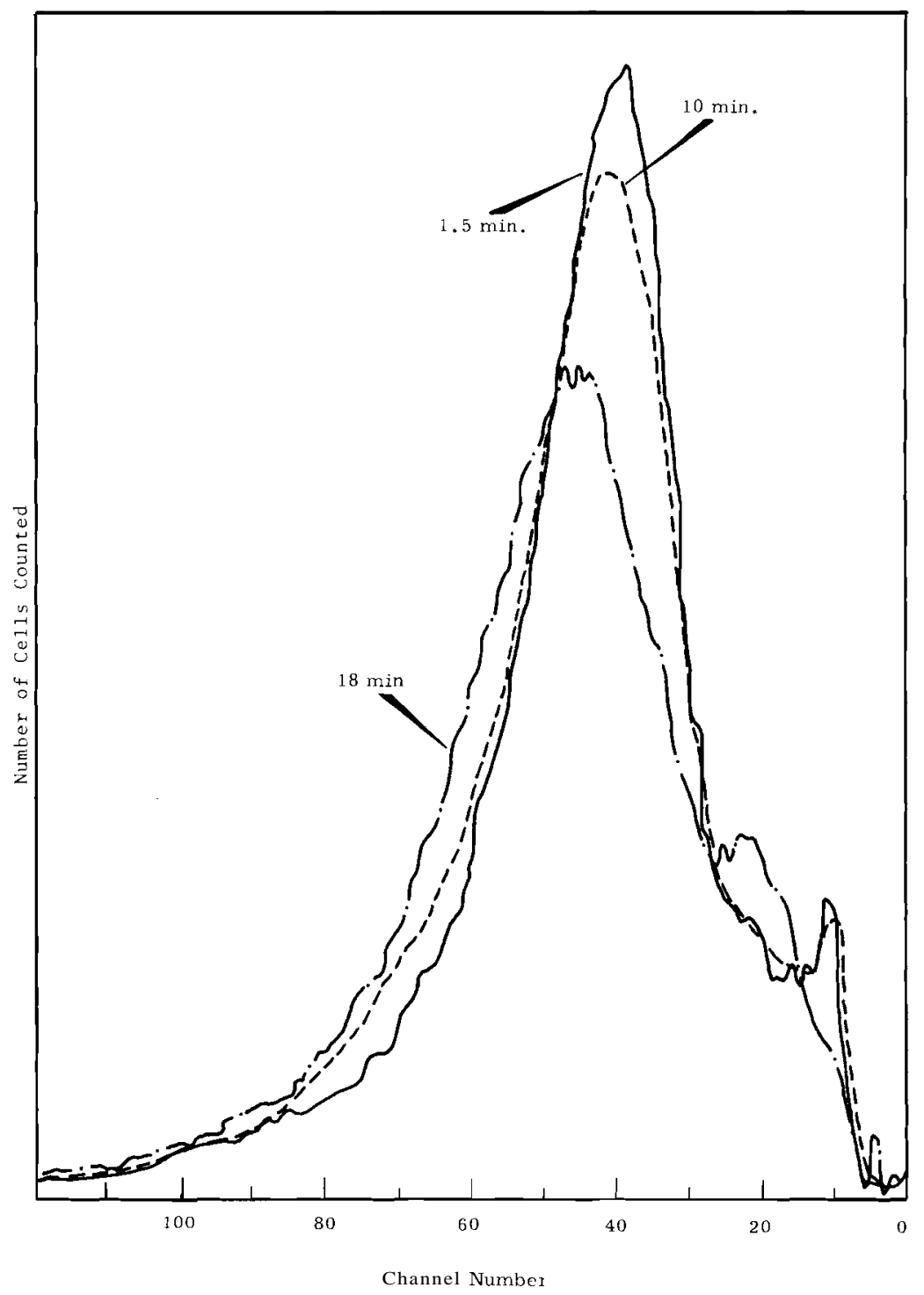

FIGURE 60. Effect of Delay Between Dilution and Counting on Cell Size Distribution Determination. Buffered Saline

swelling during the counting interval even in suspensions counted immediately upon dilution. The rate of swelling is also noted to be greater in normal saline than in saline buffered to physiologic pH. Our work agrees with that of Lushbaugh, et a1. (52) in emphasizing the importance of $\mathrm{pH}$ on the erythrocyte size distribution.
These results demonstrate the problems inherent in comparison of distribution curves obtained at one site with those obtained at another. In fact, reproducibility is difficult to maintain from day to day using the same instrument. It is obviously necessary that to allow quantitative correlation 
with results from Coulter Counter studies, the conditions of the experiment,
$\mathrm{pH}$, salinity, dilutions, counting times, aperture dimensions, and Coulter Counter settings, must be recorded and reported.

\section{RADIATION-INDUCED HEMOLYSIS OF ERYTHROCYTES-W. D. Felix}

Previous reports on the effect of ionizing radiation on erythrocytes in physiological saline have described the general kinetic scheme $(53,54)$ and have attempted to formulate equations to describe the diffusion-controlled processes leading to cell swelling. (55) These reports indicated the value of techniques developed in demonstrating the effects of radiation on a model biological system. Further efforts have now been applied to improve the analytical techniques used to follow the radiation-induced hemolysis of red cells. The techniques were utilized to examine the application of the kinetic formulations to cells having received higher radiation doses than used previously.

Blood was obtained by venipuncture of selected control anaimals. Heparin was used as anticoagulant. Cells were washed three times in buffered saline and stored at $4{ }^{\circ} \mathrm{C}$. Sample tubes for irradiation studies were thoroughly cleaned, filled with doubly distilled water and irradiated and finally flamed to remove organic residues. Temperature control during irradiation was ef: fected by a Dewar flask containing 37 C water. Since most irradiations were for times long enough for cell settling to occur, it was found necessary to mix the cell suspension by oxygen bubbling. The volume ratio of red blood cells to buffered saline was usually about $1: 100$. Following irradiation in the Gammacell ${ }^{60}$ Co source, $0.1 \mathrm{ml}$ of the $3 \mathrm{ml}$ suspension was removed with a micro-syringe, diluted with up to 20 parts buffered saline, and centrifuged in a microcentrifuge. Hemoglobin concentration in the supernatant solution was determined at $409 \mathrm{m \mu}$, using a Beckman DU spectrophotometer with a micro-cell attachment.

The radiation-induced hemolysis of sheep erythrocytes in buffered saline at various dosages is characterized by the curves shown in Figure 61. The vertical lines indicate the variation between replicate samples of this particular set. In suspensions in which mixing was not complete, the variation between samples was large; however, in the set shown, where mixing was complete, the variation between samples is within the limits of the spectrophotometric method.

Reproducibility of hemoglobin analyses made concurrently from the same sample depended on the type of centrifuge cup used. Intact cells adhered to the sides of polystyrene and polyethylene tubes. Celluloid tubes were found to be most satisfactory in use but are apparently no longer available in the size required for micro-centrifugation. The erratic behavior of the experimental points noticeable in Figure 61 for hemoglobin concentrations 


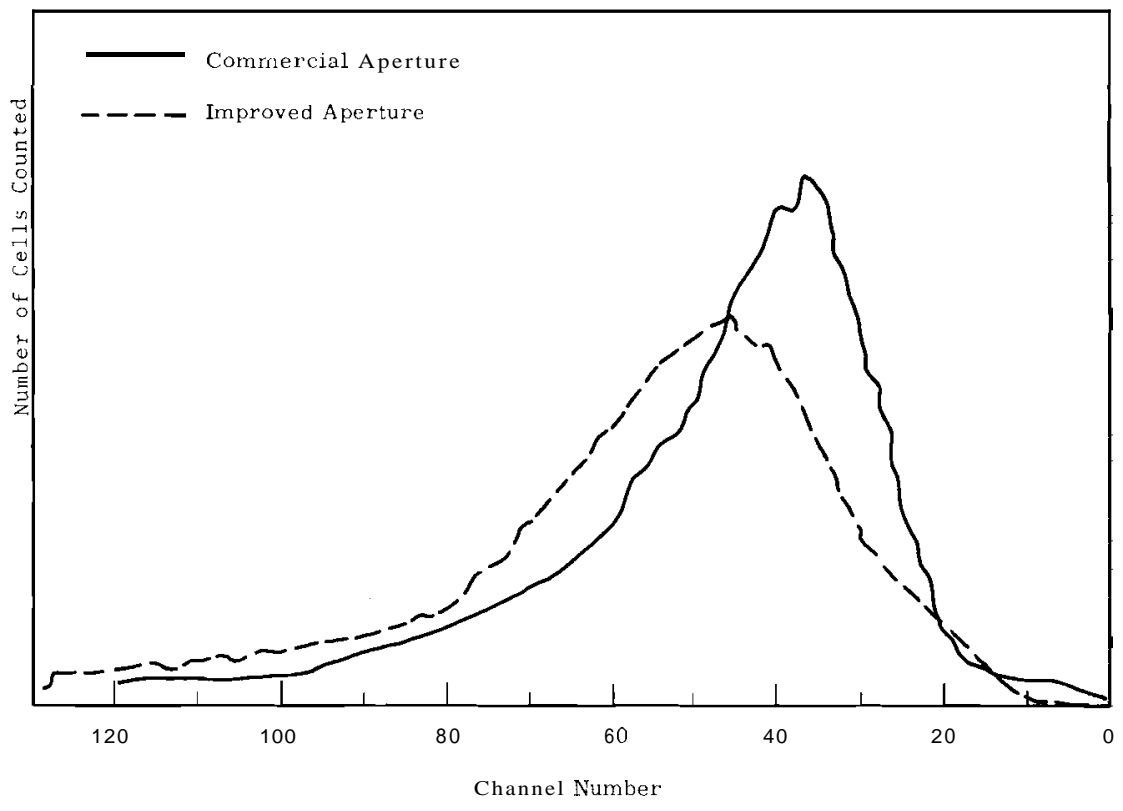

FIGURE 61. Representative Example of Erythrocyte Hemolysis Techniques. Post Irradiation-Induced Hemolysis of Sheep Cells Irradiated at $37^{\circ} \mathrm{C}$. (Dose Rate 2500 Rads/min)

near zero can be attributed to cells adhering to the sides of the tubes during centrifugation.

Cell settling during irradiation was a major factor with respect to reproducibility among samples irradiated at the same dose level. As the cells repacked in the bottom of the tube during irradiation, the effective free radical attacks received by each cell in the packed region was less than that received by the cells remaining in suspension. Other investigators have also observed this and have attributed a $20 \%$ protection effect to the close packing of cells. They also showed that the observed protection was due only to a decrease in the volume of water around the erythrocytes and not to inhibition of metabolic processes. (56) Although contributing to frothing, oxygen bubbling was found to be an effective means of maintaining a uniform cell suspension during irradiation.

The realtionship between absorbed dose and $\tau$, the time required for half hemolysis, is presented in Figure 62 . As expected from earlier results, (53)

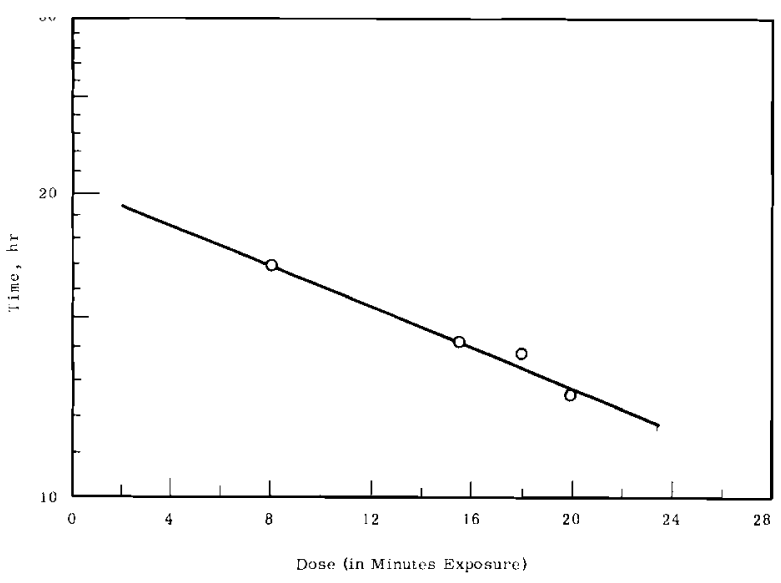

FIGURE 62. Time to Half Hemolysis as

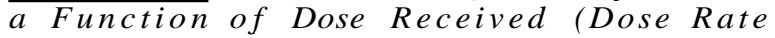
2500 Rads/min) 
the relationship may be described by the exponential function:

$$
\text { Dose }=B \ln (K / \tau)
$$

where $B$ and $K$ are experimental parameters The result demonstrates the validity of the equation at higher dosages than used previously. $(53,54)$

It was noted in this set of samples, as in others, that at the high dosages an amorphous precipitate appeared. Con currently, as the dose increased, the hemoglobin present in the supernatant at complete hemolysis decreased. This is demonstrated in Figure63 where the

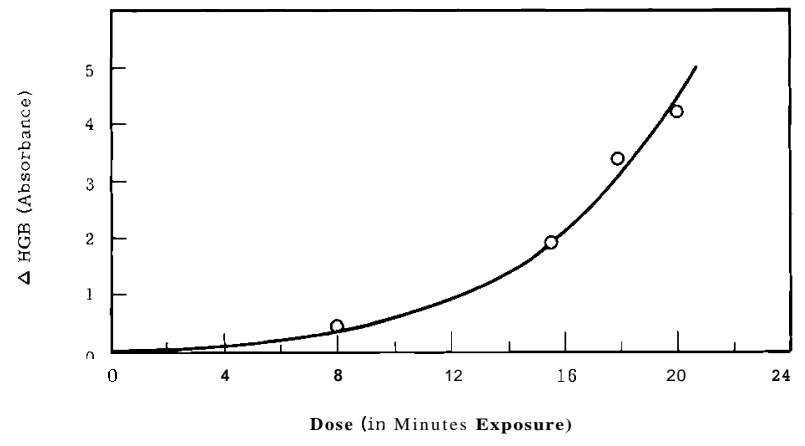

FIGURE 63. Irreversible Denaturation of Hemoglobin expressed as a Function of Difference in Absorbance Units of Completely Hemolyzed Control and Irradiated Samples (Dose Rate 2500 Rads) min)

difference between hemoglobin absorbance of totally hemolyzed control and irradiated samples is plotted against dose received by the irradiated samples. The difference as plotted is a measure of the hemoglobin precipitated by irradiations. The residue which is formed may occur as a result of: reaction of hemoglobin with irradiation decomposition products of the intraor-extra-cellular constituents; decomposition of free hemoglobin by free radical attack or by direct hit; or, free radical attack on hemoglobin followed by polymerization. For valid determination of the effect of biological protective agents it is necessary to control the dosage to cell concentration ratios such that the system is in the lower flat portion of the curve in Figure 63.

With the techniques developed to follow the hemolysis of irradiated erythrocytes, a quick, convenient, and quantitative biological testing ground for radiation protective agents is available. Erythrocytes are readily learned by skilled technicians. Thus a complete evaluation of protective proficiency can be made in a few days. Dose to cell concentration ratios should be sufficient to allow complete hemolysis in about 36 to $48 \mathrm{hr}$ while not leading to excessive deposition of hemoglobin-bearing sediment.

\section{EFFECT OF CHLORIDE ION ON THE RADIOLYSIS OF ERIOGLAUCINE SOLUTIONS-}

W. D. Felix

The radiation chemistry of chloride solutions at $\mathrm{pH}$ from 0 to 7 has been the subject of two recent articles. It has been suggested that the overall reaction,

$$
\mathrm{OH}^{\prime}+2 \mathrm{Cl}^{-} \rightarrow \mathrm{OH}^{-}+\mathrm{Cl}_{2}^{-}
$$

is highly $\mathrm{pH}$ dependent and is rate $\mathrm{lim}$ ited by a reaction step (51) involving a singly charged negative ion. 
The rate of production of $\mathrm{Cl}_{2}^{-}$is independent of oxygen concentration and is first order in hydrogen ion, chloride ion, and hydroxyl radical concentrations. From the observation that addition of hydrogen peroxide, and electron scavenger, had no effect on the production of $\mathrm{Cl}_{2}^{-}$, Anbar and Thomas (57) concluded that $\mathrm{C}_{2}$ was generated only in the spur regions.

Anbar and Thomas considered three plausible mechanisms involving first order dependence on chloride ion, hydrogen ion, and hydroxyl radical concentrations:

$$
\begin{aligned}
& \mathrm{H}_{3} \mathrm{O}^{+}+\mathrm{Cl}^{-} \rightarrow \mathrm{HCl}+\mathrm{HOH} \\
& \mathrm{HCl}+\mathrm{OH}^{\prime} \rightarrow \mathrm{Cl}^{*}+\mathrm{HOH} \\
& \mathrm{OH}^{\prime}+\mathrm{Cl}^{-} \text {- } \mathrm{HOCl}{ }^{*} \\
& \mathrm{HOCl} 1^{-}+\mathrm{H}^{+} \rightarrow \mathrm{Cl}^{+}+\mathrm{HOH} \\
& \mathrm{OH}+\mathrm{H}_{3} \mathrm{O}^{+} \rightarrow \mathrm{H}_{2} \mathrm{O}^{+}+\mathrm{HOH} \\
& \mathrm{H}_{2} \mathrm{O}^{+}+\mathrm{Cl}^{-} \rightarrow \mathrm{Cl}^{\bullet}+\mathrm{HOH} \text {. }
\end{aligned}
$$

None of the three mechanisms discussed was found free from objection. Ward and Myers (58) assumed that in the presence of $\mathrm{NaCl}$ in acid solution, the hydroxyl radical was converted to a different species which reacted preferentially with the double bonds of thymine Utilizing the $\mathrm{OH}$ scavenging properties of ethanol, and by varying $\mathrm{pH}$ and chloride ion concentration, they obtained results which they felt indicated a third order mechanism:

$$
\mathrm{OH}^{\prime}+\mathrm{H}^{+}+\mathrm{Cl}^{-} \rightarrow \mathrm{Cl}+\mathrm{HOH}
$$

with a rate constant of $7.6 \times 10^{9}$ liter ${ }^{2}$ mole $e^{-2} \mathrm{sec}^{-1}$.

The radiation chemistry of chloride ion was examined by competitive reaction techniques using erioglaucine as the competitive indicator. The proposed termolecular mechanism of Ward and Myers (58) was imposed and the results at neutral pH were compared with their results at low $\mathrm{pH}$. The $\mathrm{pH}$ of the solutions was determined with a Beckman Model G pH-meter before and after irradiation. No changes in $\mathrm{pH}$ of these unbuffered systems were detected beyond the normal instrumental error limits.

Solutions $6 \times 10^{-6} \mathrm{M}$ in erioglaucine in doubly distilled water were made up with various $\mathrm{NaCl}$ concentrations from 0 to 1.0M. The solutions were irradiated for $1.5 \mathrm{~min}$ in a Gammacell ${ }^{60} \mathrm{Co}$ source delivering $2680 \mathrm{rads} / \mathrm{min}$. Radiation-induced bleaching of the erioglaucine was determined by spectrophotometric absorbance measurements at $631 \mathrm{m \mu}$. The results are displayed in Figure 64 where the absorbance differences ( $A_{0}$ - A) between the nonirradiated and the irradiated solutions are

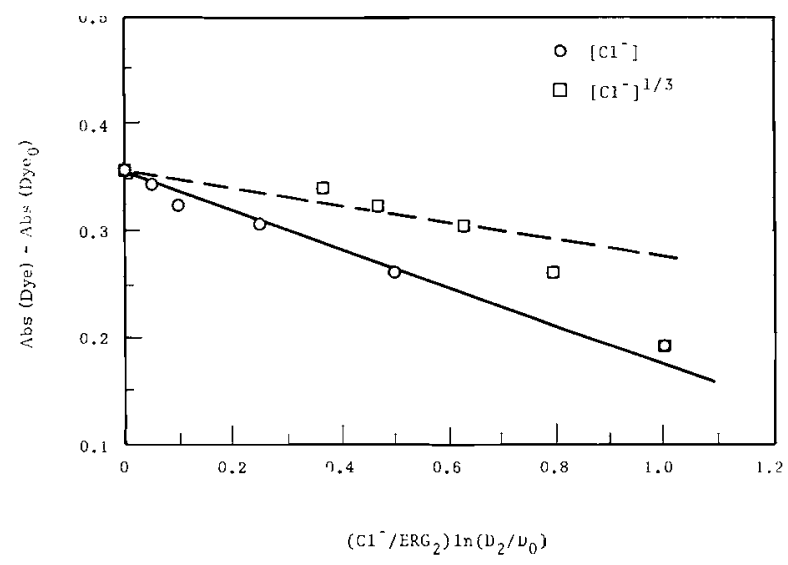

FIGURE 64. Dependence of Radiation Induced Bleaching of Erioglaucine on $\mathrm{Cl}^{-}$Concentration

plotted against $\left[\mathrm{Cl}^{-}\right]$and $\left[\mathrm{Cl}^{-}\right]^{1 / 3}$. Data were corrected for the increase in absorbed dose due to the high sodium chloride concentration.

Allen (59) in his review work on the radiation chemistry of water, discussed 
the empirical relationship whereby hydrogen and hydrogen peroxide yields in salt solutions could be plotted against the cube root of the concentration of the anion concentration to yield a straight line. This relationship was shown to hold until the yield fell to about 0.6 of its limiting value. Anbar and Thomas noted a dependence of $\mathrm{Cl}_{2}^{-}$formation on the cube root of the $\mathrm{Cl}^{-}$concentration. In Figure 64 the cube root curve is not linear beyond about 0.85 of its limiting value. However, the first order dependence of erioglaucine bleaching is linear.

Under the termolecular hypothesis, the reactions of erioglaucine and chloride ion in competition for hydroxyl radicals are written,

$\mathrm{OH}^{\prime}+\mathrm{ERG} \rightarrow$ ERG_products $\left(\mathrm{k}_{1}\right)$

$\mathrm{OH}^{\cdot}+\mathrm{Cl}^{-}+\mathrm{H}^{+} \mathrm{Cl}^{\cdot}+\mathrm{HOH}\left(\mathrm{k}_{2}\right)$

At the high concentration of $\mathrm{C} 1$ used, the reaction

$$
\mathrm{Cl}^{-}+\mathrm{Cl} \cdot \rightarrow \mathrm{Cl}_{2}^{-}
$$

is assumed to remove all of the chlorine atoms.

Assuming a steady state concentration of $\mathrm{OH}$ radicals and a constant $\mathrm{C}^{-}{ }^{-} \mathrm{Con}-$ centration, consideration of the competition kinetics leads to the equation $\frac{\mathrm{D}_{1}}{\mathrm{D}_{2}}=1+\frac{\mathrm{k}_{2}}{\mathrm{k}_{1}} \cdot \frac{\left(\mathrm{Cl}^{-}\right)(\mathrm{H}+)}{\left(\mathrm{ERG}_{2}\right)} \cdot \ln \frac{\left(\mathrm{D}_{2}\right)}{\left(\mathrm{D}_{\mathrm{O}}\right)}$

where the optical densities of erioglaucine before irradiation, following irradiation, and following irradiation in the presence of $\mathrm{NaCl}$ are $\mathrm{D}_{0}, \mathrm{D}, \mathrm{D}_{2}$ respectively, and $\left(E_{2} G_{2}\right)$ is the concentration of erioglaucine after irradiation in the presence of $\mathrm{NaCl}$. Plotting $\left(\mathrm{Cl}^{-}\right) /$ $\left(\mathrm{ERG}_{2}\right)$ in $\left(\mathrm{D}_{2} / \mathrm{D}_{0}\right)$ vs $\mathrm{D}_{1} / \mathrm{D}_{2}$ results in the linear curve shown in Figure 65 having a slope of 0.42 . The $\mathrm{pH}$ of the doubly distilled water used for the irradiation was 6.2 corresponding to a hydrogen

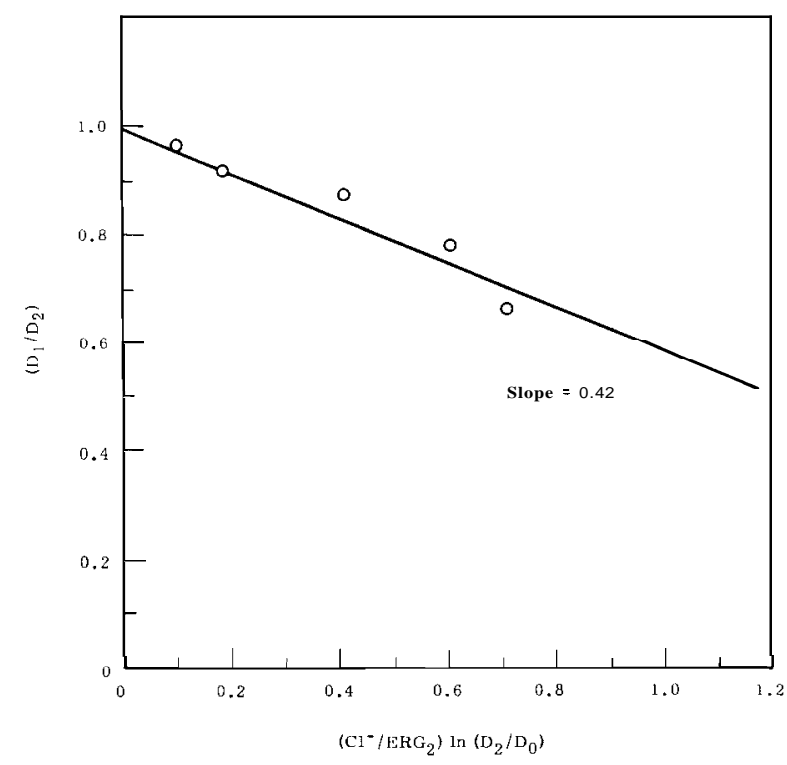

FIGURE 65. Kinetic Plot of Radiation Induced Bleaching of Erioglaucine in the Presence of Chloride

ion concentration of $6.3 \times 10^{-7} \underline{\mathrm{M}}$. Using a reported value for $k_{1}$ of $4.7 \times 109$ $\underline{M}^{-1} \sec ^{-1(60)}$ and with an absorptivity coefficient for erioglaucine of $1.1 \times 10^{5}$, the overall rate constant for $\mathrm{Cl}_{2}^{-}$formation is $2.8 \times 10^{10}$. This result is higher than that of Ward and Myers, (58) $7.6 \times 10^{9} \underline{\mathrm{M}}^{2} \mathrm{sec}^{-1}$, obtained at low $\mathrm{pH}$.

Anbar and Thomas (57) thought it unlikely that an activated complex of the composition $\mathrm{Cl}^{-}-\mathrm{OH}-\mathrm{H}_{3} \mathrm{O}^{+}$could be formed by a random three body collision at a high enough rate to explain the rapid $\mathrm{Cl}_{2}^{-}$formation. The results presented here are not sufficient to refute their argument nor definitely to preclude one of the three bimolecular mechanisms presented earlier. 
The dependence of the formation of $\mathrm{C}_{2}^{-}$on the cube root of the chloride ion concentration has been well established. $(57,59)$ The failure of the cube root test in this experiment may indicate the assumption to be incorrect that
$\mathrm{Cl}_{2}^{-}$does not react with erioglaucine. Correction for $\mathrm{Cl}_{2}^{-}$reaction would lead to the calculation of a greater initial $\mathrm{Cl}_{2}^{-}$yield and would also cause an increase in the calculated termolecular rate constant.

\section{ELECTRON SPIN RESONANCE DETECTION INSTRUMENTS-R. N. Diebel}

Back-diode microwave detectos are recommended for use at $400 \mathrm{~Hz}$ modulation. They provide about a lo-fold improvement in signal-to-noise ratio over $1 N 23 E$ point cpntact detectors. Calculation of electron spin resonance (ESR) signal amplitude increases at lower frequencies in proportion to $\nu^{-3.58}(\nu=$ radio frequency). Thus a 3 -fold decrease of frequency could provide a 50-fold increase in signal amplitude while sample volume need increase only 32 -fold.

Comparison of Microwave Detectors

Our ESR instrumentation is being used to study radicals in water solution. Since the observed signals are often only a few times the noise level, we are always striving to improve the signal-to-noise ratio. For the past 2 years we have used low noise back diode detectors. (61) These are a varieant of the Esaki or tunnel diode junction-type structure. (62) Comparison of the back diode with 1 N23E and 1 N23F point contact diodes has consistently demonstrated superior performance for the back diode when using a $400 \mathrm{~Hz}$ modulation frequency with the $9.5 \mathrm{GHz}$ microwave frequency. About a 10-fold improvement in signal-to-noise ratio is obtained by use of back diodes at $400 \mathrm{~Hz}$. When the modulation frequency is changed to $100 \mathrm{kHz}$, the back diode gives a ratio very similar to that of selected 1 N23E or 1N23F detectors. The back diode is much more rugged and gives consistent performance unmatched by the point contact diodes over periods of a year or longer. Very recently we have used some 1 N23G point contact diodes and find them superior to their predecessors in the 1 N23 series when tested at $100 \mathrm{kHz}$. No test has been made with the $1 \mathrm{~N} 23 \mathrm{G}$ at $400 \mathrm{~Hz}$.

The lower intermediate frequency impedance of the back diode, $100 \Omega$ as contrasted with 500 to $800 \Omega$ for point contact types, may necessitate some changes in the coupling transformer between the microwave detector and audio amplifier. The basic theory has been published (63) and it will suffice to say that if one assumes that the transformer is perfect, that the amplifier has an equivalent noise input of $800 \Omega$ (as might be available from a good pentode amplifying stage), and that the amplifier input impedance is about $10^{5} \Omega$, then the input transformer should have a turns ratio of about $1: 18$. The Varian V4500 Electron Spin Resonance spectrometer, which serves as the basis for our equipment used a $1: 10$ ratio for the input transformer. We have modified this transformer (after 
sawing open the copper case) by center tapping the input winding ( it is only 11 turns and hence easily rewound). This gives a 1:20 ratio on each side of the center tap.

To simultaneously retrieve the 100 $\mathrm{kHz}$ signal along with $10 \mathrm{kHz}$ and 400 $\mathrm{Hz}$ (or lower frequencies) we have used two transformers in series as shown in Figure 66. A balanced homodyne detection system has been used since it greatly reduces klystron FM noise. The split primary windings of the low frequency transformer are connected to each end of the $100 \mathrm{kHz}$ transformer. Low impedance, low noise detectors require a low resistance dc return, typically $10 \Omega$ or less, to prevent excess noise. The turns ratio of the low frequency transformer can also be larger, between 1:50 and $1: 100$ since the audio frequency vacuum tube amplifier will have an input impedance of around $10^{7} \Omega$.
One important detail in maintaining good signal-to-noise ratio in these high-gain detection systems is to provide a break in the dc path between the microwave detector mounts and the rest of the microwave system. This prevents formation of ground loops between the receiver and transmitter sections of the spectrometer.

A very recent development worthy of note is the introduction of a new type of noise microwave detector diode, the "hot carrier" diode. (64) We hope that the manufacturer will soon find it possible to make a diode package which performs as well at $9 \mathrm{GHz}$ as do the prototypes operating at 1 to $2 \mathrm{GHz}$.

Dependence of Electron Spin Resonance Signal Amplitude Upon Radio Frequency

In our studies of radicals induced in aqueous solutions, we often have available quantities of material of

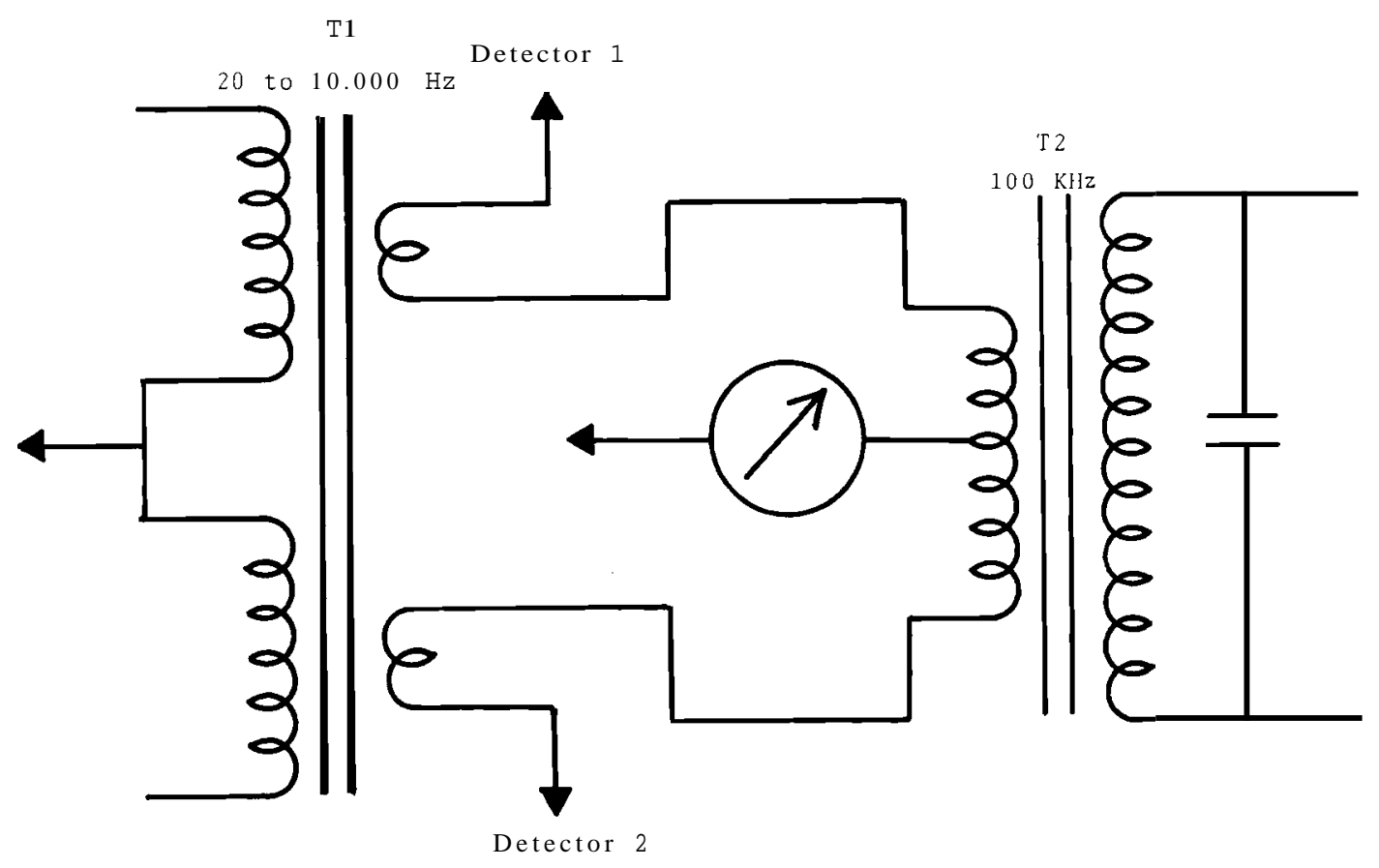

FIGURE 66. Transformer Connections for Preamplifier Input of ESR Spectrometer 
the order of $100 \mathrm{ml}$. We can therefore provide sample enough to fill a rather large microwave cavity. This has led us to re-examine the relationships between electron spin resonance signal amplitude, cavity configuration, and the radio frequency for spin resonance.

The factors which determine the signal amplitude operate as follows: (65)

$$
\text { Amplitude } \propto \mathrm{x}{ }_{n} \mathrm{P}^{0.5} \mathrm{DV}_{\mathrm{S}}
$$

where

$$
\begin{aligned}
& x^{\prime \prime}=\text { rf magnetic susceptibility } \\
& \text { per gram } \\
& \eta=\text { filling factor, i.e., } \\
& Q=\text { cavity quality factor } \\
& \mathrm{P}=\text { radiofrequency power ab- }
\end{aligned}
$$

For radicals in dilute, low viscosity solutions, and in the absence of line broadening due to chemical reactions, these factors are related to the radio frequency, $v$, as detailed below: (65-67)

$$
\begin{aligned}
x^{\prime \prime} & v \\
Q \propto & v^{-1 / 2} \\
\eta \propto & v^{-1 / 6} \text { is the result of } \\
& n \propto\left(\varepsilon^{\prime \prime}\right)-1 / 3 v^{1 / 6} \text { in a } \\
& \text { rectangular TE102 cavity } \\
& \text { and of } \varepsilon^{\prime \prime} \propto v \text { in the fre- } \\
& \text { quency range from about } \\
& 60 \text { MHz up to } 9 \text { GHz ( } \varepsilon^{\prime \prime} \\
& \text { is the loss portion of } \\
& \text { dielectric constant. ( } 66 \text { ) } \\
& \text { This applies only for a } \\
& \text { thin slab of sample in a } \\
& \text { rectangular TE102 cavity.) }
\end{aligned}
$$

$$
\begin{aligned}
n \propto & V_{s} / V_{c} \text { where } V_{c} \text { (volume } \\
& \text { of the cavity and sample) } \\
& \text { is a thin slab placed at } \\
& \text { a node of microwave mag- } \\
& \text { netic field in the cavity. }
\end{aligned}
$$

Combining these last two relations yields

$$
\begin{gathered}
\mathrm{V}_{\mathrm{s}}=\mathrm{nV}_{\mathrm{c}}=\mathrm{v}-1 / 6 \mathrm{~V}_{\mathrm{C}} \text {, however } \\
\mathrm{V}_{\mathrm{c}} \propto \mathrm{v}^{-3} \text { so } \mathrm{V}_{\mathrm{s}} \propto \mathrm{v}^{-3.16} \\
\mathrm{P}^{1 / 2} \propto \mathrm{v}^{-3 / 4} \text { for samples exhib- }
\end{gathered}
$$

iting power saturation, as is the case for most organic radicals, and we choose to operate at the brink of saturation. (66)

Combining all these terms leaves v with an exponent having the sum

$$
1-\frac{1}{6}-\frac{1}{2}-\frac{3}{4}-3.16=-3.58,
$$

or ESR Signal Amplitude $\propto v^{-3.58}$

This expression indicates that if the radio frequency electron spin resonance were to be reduced from the commonly used $9 \mathrm{GHz}$ region down to $3 \mathrm{GHz}$, the signal amplitude would increase about 50 fold. Such a tremendous increase in signal amplitude would be a real breakthrough in examination of radiation-induced free radicals and of other paramagnetic materials where sufficient volume of sample is available. Table VI presents a comparison of the operating parameters calculated for three different frequencies on the basis of the theory.

Performance of electron spin resonance studies at frequencies lower than the $9 \mathrm{GHz}$ now commonly used could run into some problems in spectral resolution when the operating frequency is not much larger than 
TABLEVI. Calculated Variation of Electron Spin Resonance with Radio Frequency for Aqueous samples

Frequency $\mathrm{GH}_{z}$

Magnetic field, Oe

Cavity Dimensions, (a) cm

Sample Volume, (b) $\mathrm{m} 1$

Relative ESR Signal Amplitude

Relative Sensitivity,

spins per unit volume

Relative Modulation (c)

Driving Power

Optimum Sample Thickness, mm
9.51

3370

$2.2 \times 1.0 \times 4.1$

0.5

1

1

1

0.25
3.17

1230

$6.7 \times 3.0 \times 12$

16

51

1.6

1.9

25

1.64

(a) Height times width times length for rectangular TE102.

(b) Sample could be smaller; in fact, reduction of height to occupy central $50 \%$ of cavity would reduce filling factor for radio frequency magnetic field by only $30 \%$.

(c) Assumed to be proportional to area of broad side of cavity.

the magnetic energy separations involved in the fine structure. For organic radicals in solution these fine structure interactions are usually around 25 gauss or less and so one might suppose that operation at 250 gauss or higher, i.e., $650 \mathrm{MHz}$ or higher, would still enable resolution of the larger nuclear hyperfine interactions.

In conclusion, it appears that the amplitude increase available by operating with a larger cavity at a lower frequency merits immediate attention. The theory, as exemplified in Table VI, indicates that de- tection of radicals with unpaired electrons could be achieved at concentrations one-fiftieth or less than presently achieved. Problems would undoubtedly arise in obtaining materials of construction of sufficient purity to meet the demands of a 100 fold reduction in detection limit. We need this decrease in detection $1 \mathrm{imit}$ to be able to search for radicals produced in living systems by non-lethal doses of radiation. No means of achieving such a large increase in electron spin resonance signal seems conceivable, other than this one of using larger samples at lower radio frequencies.

${ }^{60}$ CO IRRADIATOR FLOW SYSTEM FOR ELECTRON SPIN RESONANCE- R. N. Diebel and D. R. Kalkwarf

Considerable alteration of the existing flow/irradiation system (68) has been made to provide greater freedom from contamination and better con- trol over the rate of flow (Figure 67). Connections and valving are now constructed of glass. A glass irradiation coil was assembled which can be moved 


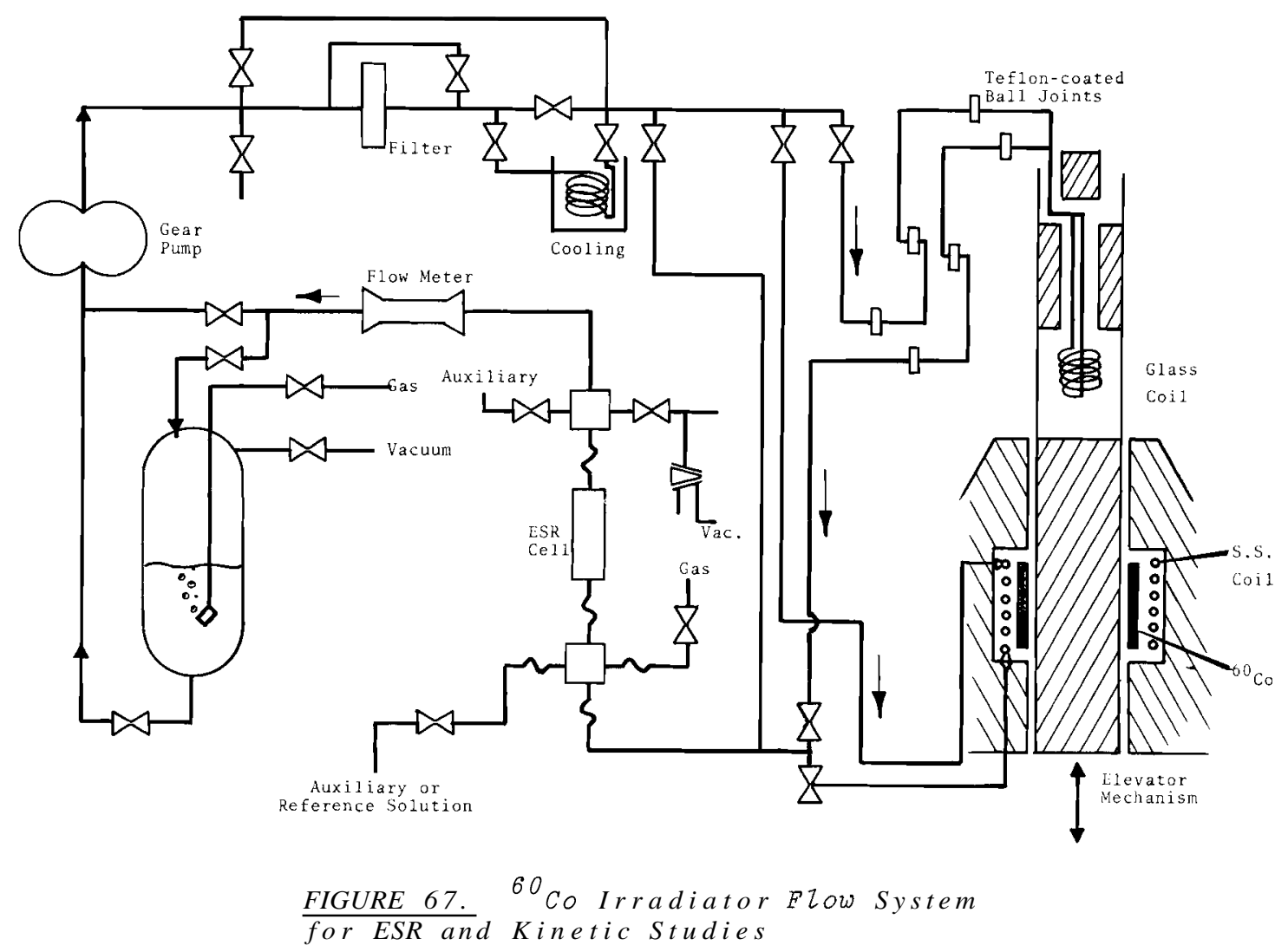

in and out of the irradiation zone as needed. The flow is controlled by a stainless steel gear pump equipped with external mechanical seal and extended stuffing box to provide high vacuum seal for the shaft. The pump is driven from a constant speed motor through a variable ratio gear box, and an electrically controlled magnetic clutch is used in a manner which limits the torque transmission, thus protecting the gear pump. The system is being evaluated in connection with our continuing studies on irradiation-produced free radicals.

LONG-LIVED ORGANIC RADICALS FORMED IN IRRADIATED AQUEOUS SOLUTION-D. R.

Kalkwarf and R. N. Diebel

Although many free-radical reactions proceed rapidly in the liquid phase, the significant effect which chemical structure has upon reaction rates leads one to suspect the existence of radicals which are relatively stable even when rapid diffusion is possible. Such radicals would be of particular importance in radiobiology, since if their precursors were naturally present in an organism or introduced before irradiation they could slow down the rate of 
radiation damage and thus provide more opportunity for repair and postirradiation therapy. The present study shows that several radiation-induced radicals are relatively stable in aqueous solution and indicates how they are formed.

Solutions of the organic compounds were irradiated at room temperature in Pyrex containers placed within the sample compartment of a Gammace11 200. The absorbed dose rate was 3000 rads/ min as determined by chemical dosimetry. When molecular oxygen was to be excluded from a solution, argon was bubbled through it for 20 min with the solution in a glove box filled continuously with a nitrogen atmosphere.

The containers were then closed with ground-glass stoppers lightly sealed with silicone grease and transferred to the irradiation zone. Following irradiation, the solutions were returned to the inert atmosphere of the glove box where samples were prepared for examination with a Varian V-4500 Electron Spin Resonance spectrometer. The cells used were prepared by replacing the standard side-arms of Varian V-4548 aqueous sample cells with $1 \mathrm{~mm}$ I.D. vitreous silica tubing. The ends of this tubing were polished flat and the cells were made air tight by covering them with a polished silica disc, lightly greased at the edges and kept in place with a Teflon cap.

$N, N, N^{\prime}, N^{\prime}-$ Tetramethylphenylenediamine (TMPD) Solutions

Irradiation of air-saturated, aqueous solutions of TMPD at $\mathrm{pH} 4$ to 8 produced a blue solution with an electron spin resonance signal which was observable for weeks after irradiation. The measured optical absorption spectrum of the solution corresponded to that for Wurster's Blue radical cation (69)

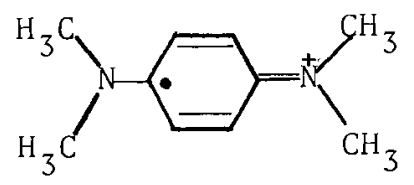

but more positive identification was obtained by electron spin resonance spectrometry. Figure 68 shows a comparison of the center portions of the spectrum for the radiation-induced radical and for Wurster's Blue synthe- sized by oxidation of TMPD with bromine. Both spectra correspond closely to published measurements on Wurster's Blue $(70,71)$ and the congruence of their center portions is evidence that the irradiated solution does not contain a mixture of two or more radiation-induced radicals.

\section{F1avin Mononucleotide (FMN)}

Some radiation-induced radicals were detected in aqueous solutions of this coenzyme whose ESR spectra depended on pH. All of these radicals reacted rapidly with molecular oxygen to give nonparamagnetic products so that oxygen was excluded during the irradiation and measurements. Figure 69 shows the spectrum of $10^{-3} \mathrm{M}$ FMN irradiated in $2 \mathrm{~N}_{2} \mathrm{SO}_{4}$. The 32 -line spectrum is very similar to those reported for AMN solutions which were chemically reduced in dilute acid, $(72,73)$. and there is no evidence that it arises from radicals with different chemical structure. Although there are many lines in these spectra, they are poorly resolved so that a precise formulation of the chemical structure of the radical is not yet possible. The spectrum could be detected for days after irradiation. 

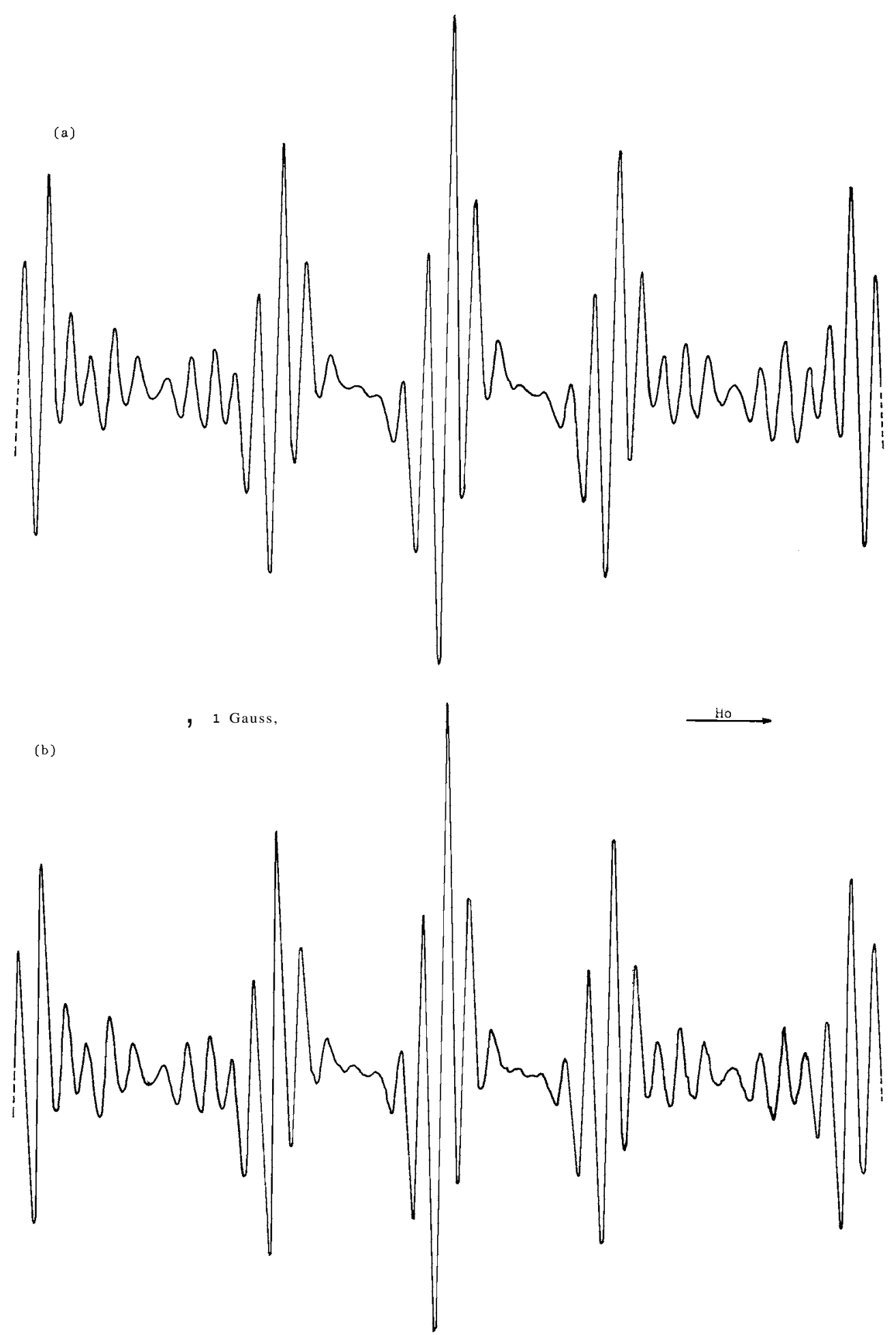

FIGURE 68. ESR Spectra of $\mathrm{fa}$ ) BromineOxidized $N, N, N, N$ - Tetrame thy Zpheny Zenediamine Solution (b) Gamma-Irradiated $N, N, N^{\prime}, N^{\prime}-$ Tetramethy Zpheny Zenediamine Solution 


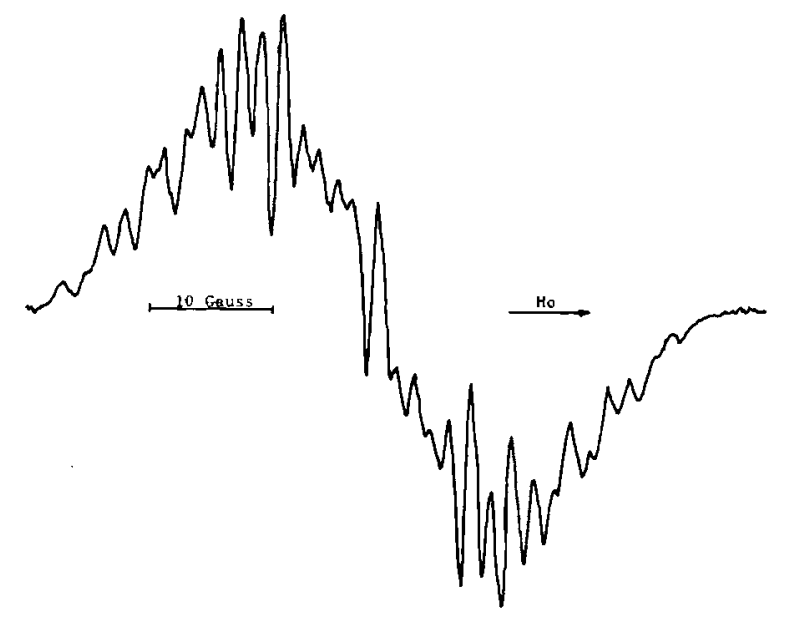

FIGURE 69. ESR Spectrum of Solution Obtained by Irradiating $0.001 \mathrm{M}$ Flavin Mononucleotide in Aqueous $2 \mathrm{~N} \overline{\mathrm{H}}_{2} \mathrm{SO}_{4}$ Containing 0.5M Ethanol.

Figure 70 shows the electron spin resonance spectrum of the radiationinduced radical formed in phosphate buffer at pH 5.8. The spectrum is different from that for the radical stable in acid solutions and it disappeared much more rapidly following irradiation.

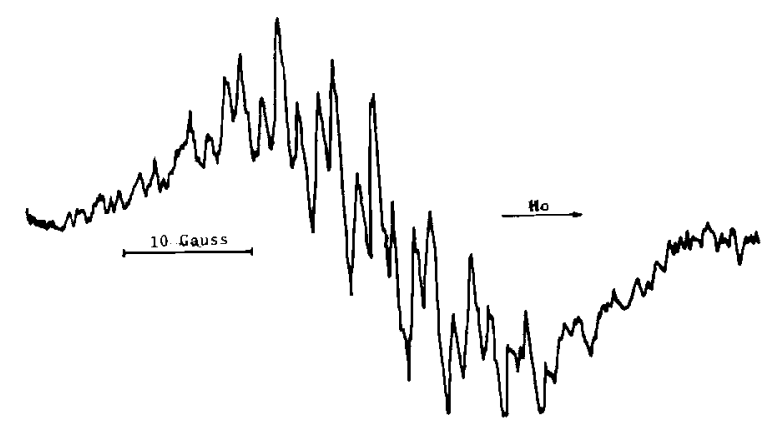

FIGURE 70. ESR Spectrum of Solution Obtained by Irradiating 0.001M Flavin Mononucleotide in Aqueous Phosphate Buffer at pH5.8 Containing 0.5M Ethanol
Due to incomplete resolution of the spectral lines, it is not possible to determine whether the number of lines or their spacings differ from those for the acid-stable radical. Addition of hydroxyl radical scavengers such as ethanol or erioglaucine increased the yield of radicals significantly, suggesting that this radical was also formed by the reducing action of species formed during water radiolysis.

Figure 71 shows the electron spin resonance spectrum of the radiationinduced radical formed in $10^{-1} \underline{\mathrm{M}} \mathrm{NaOH}$.

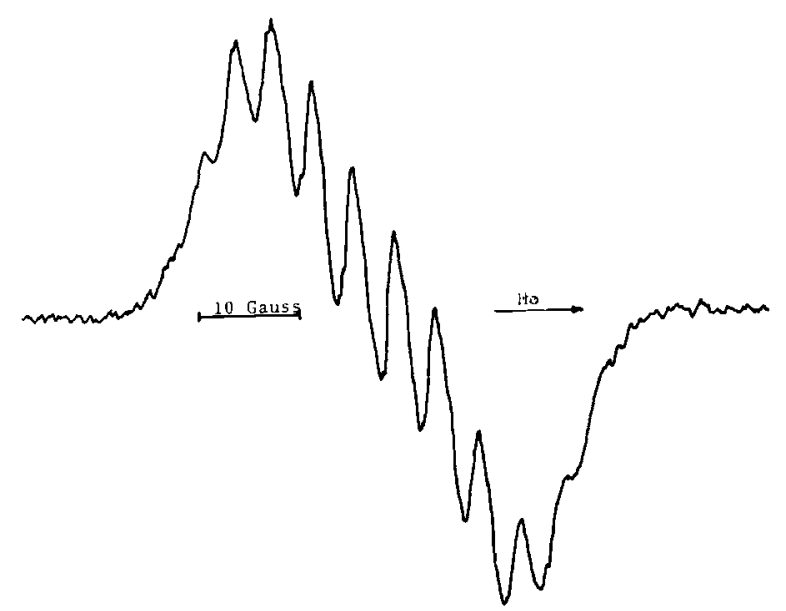

FIGURE 71. ESR Spectrum of Solution Obtained by Irradiating 0.01M Flavin Mononucleotide in Aqueous $0 . \overline{1} \mathrm{M}$ NaOH

This is quite similar to the spectrum published for FMN after reduction with dithionite in alkaline solution. (74) Again, however, the exact structure of the radical is in doubt. The radical was quite stable when protected from oxygen and could be detected for weeks following irradiation.

\section{Chlorpromazine (CPZ)}

Air-saturated aqueous solutions of CPZ, the phenothiazine drug, in $1 M$ HC1 turned pink during irradiation and were 
found to exhibit the electron spin resonance spectrum in Figure 72 . A similar spectrum has been published ${ }^{(75)}$ for the

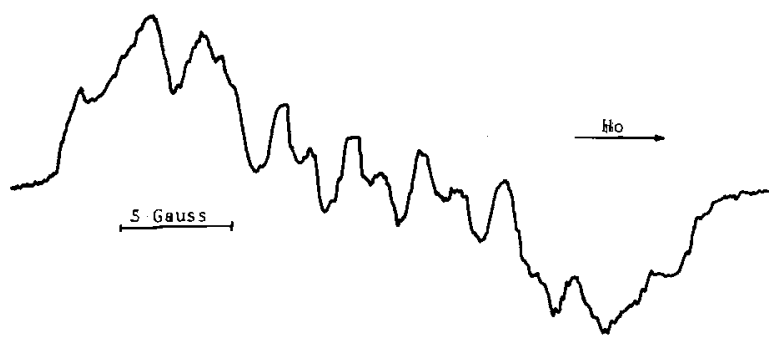

FIGURE 72. ESR Spectrum of Solution Obtained by Irradiating $0.002 \mathrm{M} \mathrm{ChZor-}$ promazine in Aqueous $1 \mathrm{M} \mathrm{HCl}$

product of electrolytic oxidation of CPZ and it was concluded that the radiation-induced radical is a product of the reaction involving CPZ with the oxidizing radicals formed during water radiolysis. The 16-line spectrum can be detected for several days in the solution and is most probably attributable to the chemical structure

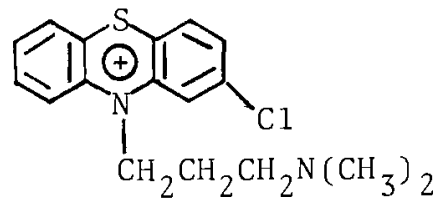

No ESR signals were observed in neutral solutions.

\section{Methylene Blue (MEB)}

Irradiation of MEB in $23 \underline{\mathrm{N} \mathrm{H}} \mathrm{SO}_{4}$, from which molecular oxygen had been removed, produced a solution with the 4-line electron spin resonance spectrum published previously. (76) Of more biochemical interest, however, was the discovery that a stable radical derived from MEB could be detected even in solutions at $\mathrm{pH} 7$ if they were saturated with sodium sulfate. The electron spin resonance spectrum of this radical is shown in Figure 73; and, while the

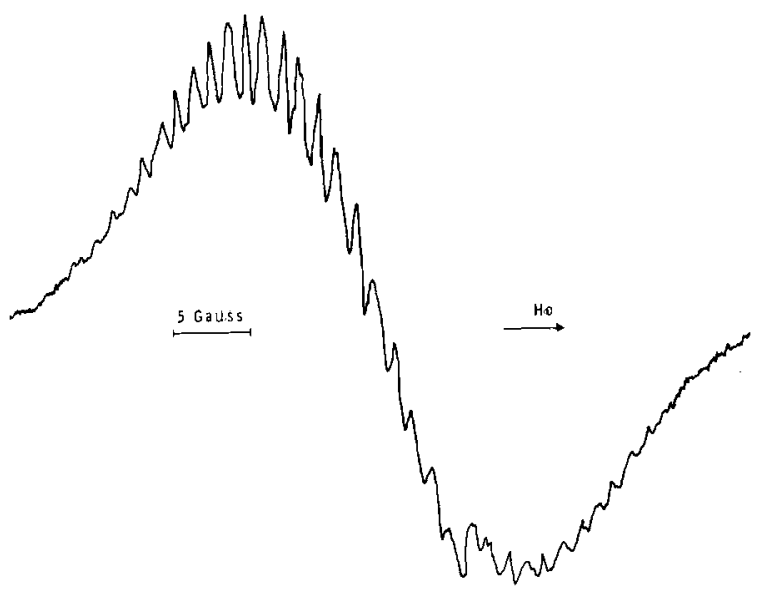

FIGURE 73. ESR Spectrum of Solution Obtained by Irradiating 0.001 M Methy. ene Blue in Saturated Aqueous Sodium Sulfate Containing 0.5M Ethanol

lines are incompletely resolved, far more than 4 lines are present. A signal can be detected for several days if the solution is not exposed to air, suggesting that the radical is a reduced form of methylene blue. Because of the broad spectrum, the unpaired electron is probably smeared over the entire ring system.

p-Nitrobenzoate (PNB)

Reexamination of oxygen-free irradiated solutions of PNB in $0.1 \mathrm{M} \mathrm{NaOH}$ failed to show the electron spin resonance spectrum previously reported ${ }^{(77)}$ for the radiation-induced radical formed in this solution. Instead, the 27-line spectrum shown in Figure 74 was obtained which corresponds exactly to the chemical structure:

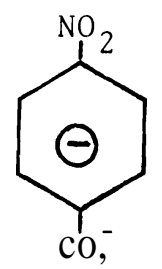


The electron spin resonance signal from this radical can be detected for about an hour after irradiation, and its for- mation seems to be well explained by addition of a radiation-induced hydrated electron to the aromatic ring system.

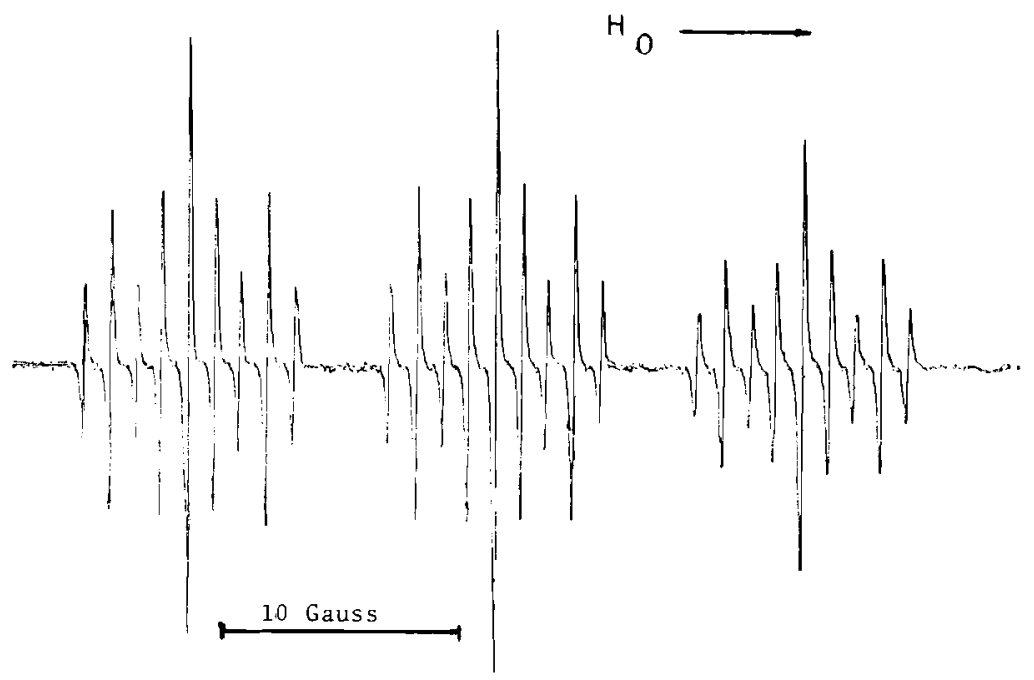

FIGURE 74. ESR Spectrum of Solution

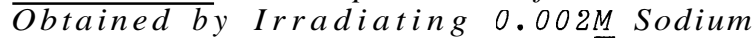
$p$-Nitrobenzoate in Aqueous 0. 1 M NaOH

REACTION KINETICS OF FREE RADICALS IN AQUEOUS SOLUTION-D. R. KaIwarf and R. N. Diebel

The discovery that long-lived organic radicals can form in several irradiated solutions $(78)$ suggests that relatively stable, yet mobile, free radicals also occur during irradiation of biological systems. Their effect on the amount of radiobiological damage is unknown as yet for while they would slow down the chain of chemical reactions initiated by irradiation and allow more time for repair mechanisms to operate, their long lifetime would also give them more opportunity to diffuse to cell sites at which critical reactions could take place. In the present study, the reaction kinetics for some of these radicals were examined in search for general principles governing their formation and disappearance so that the course of organic-radical reactions in irradiated tissue can be predicted.

Electron spin resonance spectra were used to measure the concentration of radiation-induced, organic radicals as a function of time and to detect any change in their chemical structure as reflected in the hyperfine splitting of the spectral lines. The radicals were generated either in static (78) or flowing (68) solutions by exposure to ${ }^{60}$ Co gamma radiation from a Gammacell 200 at a dose rate of 3000 rads/ min. Using the flow system, these solutions could be examined within 1 sec after irradiation, utilizing a Varian V-4500 spectrometer with $100 \mathrm{kHz}$ modulation. Radical concentrations were evaluated by recording the first- 
derivative electron spin resonance spectra at modulation amplitudes high enough to smooth out any hyperfine lines, cutting out the recorded spectra with a sharp knife, and measuring the first moments of the spectra about their centers on a moment balance. (79) These first moments, when corrected to standard settings of the instrument, were taken to be proportional to radical concentration, and the proportionality constant was obtained by measuring analogous moments for standard solutions of Wurster's Blue in water and dipheny picrylhydrazyl in benzene. The concentrations of radicals in these reference solutions were obtained from their measured optical absorbances using $A_{56 \mathrm{~m} \mu}=1.24 \times 10^{4} \mathrm{M}^{-1} \mathrm{~cm}^{-1}$ for Wurster's Blue ${ }^{(80)}$ and $A_{520 \mathrm{~m} \mu}=$ $1.15 \times 10^{4} \mathrm{M}^{-1} \mathrm{~cm}^{-1}$ for diphenylpicrylhydrazyl. (81)

One of the general principles found to govern the formation of radiationinduced organic radicals in dilute aqueous solution is that the presence of reagents that readily capture just one of the radicals formed by water radiolysis will greatly affect the radiation yield. This is due to the preponderant fraction of the radiation energy being absorbed by the water and to the conflicting chemical reactions of the resulting water radicals, some of which act as oxidizing agents and others as reducing agents. Numerous illustrations of this principle were observed but only two are described below.

The electron spin resonance spectra of the radical derived from p-nitrobenzoate ion indicated that it was the addition product of a hydrated electron to the a-electron system of the substrate. (78) Increasing the concentration of hydrogen ion in the irradiated solution increased the rate of the diversionary reaction:

$$
\mathrm{e}_{\mathrm{aq}}^{-}+\mathrm{H}_{3} \mathrm{O}^{+} \rightarrow \mathrm{H}+\mathrm{H}_{2} \mathrm{O}
$$

so that the radiation yield of the organic radical fell with decreasing $\mathrm{pH}$ as shown in Table VII. Whereas the radiation yield of the organic radical, in the absence of a hydroxyl-radical scavenger such as ethanol, is far below that of the hydrated electron it self, $G_{e}=2.8 / 100 \mathrm{eV}$ absorbed, the yield approaches this value in the presence of the scavenger.

The irradiation product of $N, N, N^{\prime}, N^{\prime}-$ tetramethyl-p-phenylenediamine was a radical-cation formed by removal of an electron from the a-electron system of the substrate, probably by reaction with hydroxyl radicals. The availability of hydrated electrons for reversing this process rose as the $\mathrm{pH}$ of the solution was increased during irradiation, and the yield of radical-cations fell as shown in Table VII.

A general principle found to affect the disappearance of radiation-induced radicals is that high ionic charge on the radicals inhibits their disappearance by radical-radical reactions. Since $\mathrm{pH}$ governs both this effect and the availability of hydrated electrons, the significance of ionic charge on the lifetimes of the radicals can be seen most clearly in their disappearance rates following irradiation.

During irradiation, solutions of p-nitrobenzoate ion and of p-nitroaniline show the production of similar radicals in which a hydrated electron has been 
TABLEVII. Effect of Hydrogen Ion and Ethanol on the Radiation Yields of Organic Free Radicals

\begin{tabular}{|c|c|c|c|}
\hline Precursor & $\mathrm{pH}$ & $\underline{\mathrm{G}(\operatorname{radica} 1 \mathrm{~s} / 100}$ & eV absorbed) \\
\hline $\begin{array}{rl}1.0 \times 10^{-3} & \frac{M}{\pi} p \text {-nitrobenzoate } \\
" & " \\
& \text { "plus } 3 \times 10^{-3} \underline{M} \text { ethanol }\end{array}$ & $\begin{array}{r}13 \\
12 \\
11 \\
10 \\
9 \\
13\end{array}$ & & $\begin{array}{l}0.9 \\
0.6 \\
0.3 \\
0.1 \\
0.0 \\
2.0\end{array}$ \\
\hline $1.0 \times 10^{-3} \mathrm{M}$ tetramethylphenylenediamine & $\begin{array}{l}7.4 \\
5.8\end{array}$ & & $\begin{array}{l}1.4 \\
3.3\end{array}$ \\
\hline
\end{tabular}

added to the n-electron system of the substrate. The disappearance rates of both radicals in oxygen-free solutions at $\mathrm{pH} 13$ following irradiation are also similar, neither being represented by a pure first- or second-order process in terms of radical concentration. But, while an empirical separation of the decay for the p-nitroaniline radicalanion into two exponential terms gives half-life values of $20 \neq 5$ and $90 \neq 15$ sec, a similar treatment for decay of the p-nitrobenzoate radical-anion gives half-life values of $5 \pm 2$ and $60 \pm 15$ min. A possible explanation for the strikingly greater stability of the $p$ nitrobenzoate radical-anion is that it is doubly charged at this $\mathrm{pH}$ whereas the p-nitroaniline radical-anion is only singly charged, i.e.,<smiles>O=[N+]([O-])c1ccc([O+])cc1</smiles><smiles>Nc1ccc([N+](=O)[O-])cc1</smiles>

and that the electrostatic repulsion between like-charged radicals limits their rate of disappearance.

Another illustration of this principle is shown by the radical formed when chlorpromazine was irradiated in aqueous solution. In $1.0 \mathrm{M} \mathrm{HC1}$, the radical was formed readily and was stable for days outside of a radiation field. In the field, it was not only formed by oxidation of chlorpromazine, probably with hydroxyl radicals, but was itself further oxidized as suggested by the data in Figure 75 .

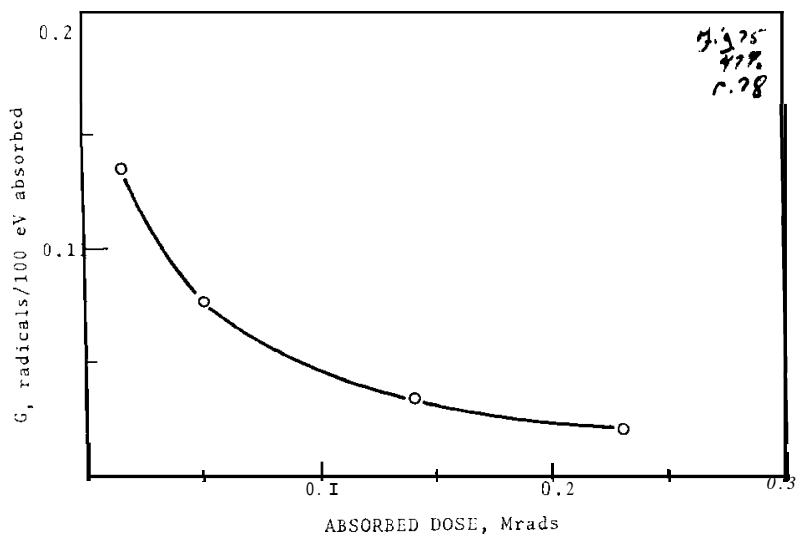

FIGURE 75. Variation of Yield for the Chlorpromazine Radical with Absorbed Radiation Dose in 1.OM HCZ Solution

At that acidity, the radical was prob-. ably in the form of the doubly charged cation:

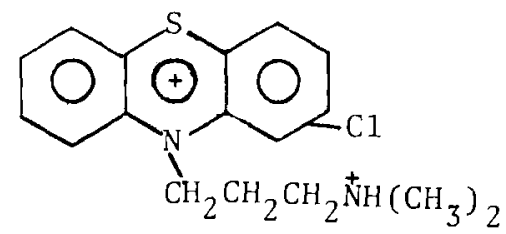


However, at pH 5.5, where the singly charged cation would be expected to form, i.e.,

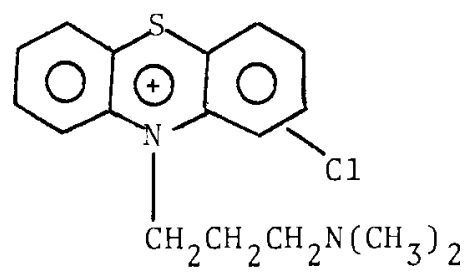

no electron spin resonance signal could be obtained after irradiation, suggesting a faster dismutation rate for this radical.

A further principle concerning the stability of radiation-induced radicals in aqueous solutions is that the presence of supposedly radiation-inert substances in the solution can significantly lengthen the lifetimes of some radicals. This principle is illustrated by the effect of sodium sulfate on the stability of radicals formed in irradiated, oxygen-free methylene blue solutions. Radiation-induced radicals derived from methvlene blue were first noted in $23 \mathrm{~N} \mathrm{H}_{2} \mathrm{SO}_{4}$ solutions, (34) and the lack of a signal at higher pH may be due to either or both of the principles mentioned above. If the solution is saturated with sodium sulfate before irradiation, however, a significant and well-resolved spectrum was obtained even at $\mathrm{pH} 7$ and was stable for days. (78) The irradiated sample was passed through a Millipore HA filter to determine whether the signal was due to suspended solids; but, while a portion of the signal intensity was lost, it was not unreasonable to attribute this to reaction with the filter.

Sodium sulfate has no known reactions with the radiolysis products of water, so other explanations of its effect must be sought. One possibility is that it raises the viscosity of the solution to a value where the rates of radicalradical reactions are inhibited. At the present, however, it seems more likelv that the intense ionic field in such solutions exerts a stabilizing influence on the radicals, and this will be explored in future experiments.

CHEMICAL PROTECTION OF AGAROSE JELLIES FROM RADIATION DAMAGED. R. Kalkwarf

The physical state of matter in many regions of living tissue is that of an aqueous jelly whose properties provide control of rigidity and the diffusion rates of metabolites within the tissues. Using aqueous jellies of agarose as tissue prototypes, it was shown earlier that addition of the artificial food coloring, erioglaucine (FD\&C Blue No. 1), allowed these polysaccharide jellies to remain firm even after absorbing up to
11 times the radiation dose which would liquefy an unprotected jelly. (83) The large protective effect of this dye as well as its relatively low toxicity to humans prompted the search described here for other protective agents among the dyestuffs authorized as food additives.

Jellies containing $0.20 \%$ agarose by weight and one of various artificial food colorings were prepared and 
irradiated. Precise control of concentration was obtained by melting together weighed quantities of a $1.00 \%$ agarose stock jelly and a standard solution of the dye in a screw-capped Pyrex test tube, tightening the cap, and then cooling the mixture rapidly to room temperature. Irradiation of these samples was accomplished in a Gammacell 200 at a dose rate of $3000 \mathrm{rads} / \mathrm{min}$. If after irradiation the sample could flow from one end of the tube to the other during gentle agitation, it was considered to be liquefied.

Several dyes were found to protect agarose jellies from radiation-induced liquefaction as shown in Table VIII, where protection is expressed quantitatively by the dose extension factor, i.e., the ratio of the liquefaction dose in the presence of the protective agent to that in its absence.

The triphenylmethane dye, FDGC Blue No. 1 (erioglaucine) and the indigo dye, FDGC Blue No. 2 were found to be the most effective although they may protect by different mechanisms. Both dyes are bleached irreversibly in irradiated aqueous solutions saturated with oxygen; but whereas ethanol, a selective hydroxyl-radical scavenger, protects FDGC Blue No. 1 from radiationinduced bleaching, it sensitizes FDEC Blue No. 2.

The critical role played by the detailed chemical structure of an additive in determining its protective ability is well illustrated by these results. The relatively poor protective agent, FDGC Green No. 2, and the relatively good protective agent, FD\&C Blue No. 1, are both triphenylmethane dyes which differ only in the position at which a sulfonic acid group is substituted in analogous aromatic rings. Similarly, FDGC Green No. 1 and FDGC Green No. 3 differ from these only in the presence or absence of ring substituents.

Practicable chemical protection of irradiated living tissue, whether it be preventing human death or preventing textural changes in radiationpasteurized food, must be possible not only with nontoxic compounds but also at concentrations of additive which can be conveniently incorporated in the system. It is thus interesting to report in Figure 76 that both FD\&C Blue No. 1 and FD\&C Blue No. 2 provide

TABLE VIII. Liquefaction Dose for $0.20 \%$ Agarose Jellies Containing $8 x 10^{-3} M$ Concentrations of Various Additives

\begin{tabular}{lcc} 
Additive & Liquefaction Dose, Mrad & Dose Extension Factor \\
\cline { 2 - 3 } None & 0.63 & 1.0 \\
FD\&C Green No. 1 & $\mathbf{1 . 1}$ & 1.5 \\
FD\&C Green No. 2 & $\mathbf{1 . 1}$ & 1.5 \\
FD\&C Yellow No. 5 & $\mathbf{1 . 1}$ & 2.5 \\
FD\&C Green No. 3 & 1.5 & 3.2 \\
FDGC Yellow No. 6 & 2.0 & 4.4 \\
FD\&C Re? No. 3 & 2.8 & 4.4 \\
FD\&C Violet No. 1 & 2.8 & 14 \\
FD\&C Blue No. 1 & 9.1 & 16 \\
FD\&C Blue No. 2 & 9.3 & \\
\hline
\end{tabular}




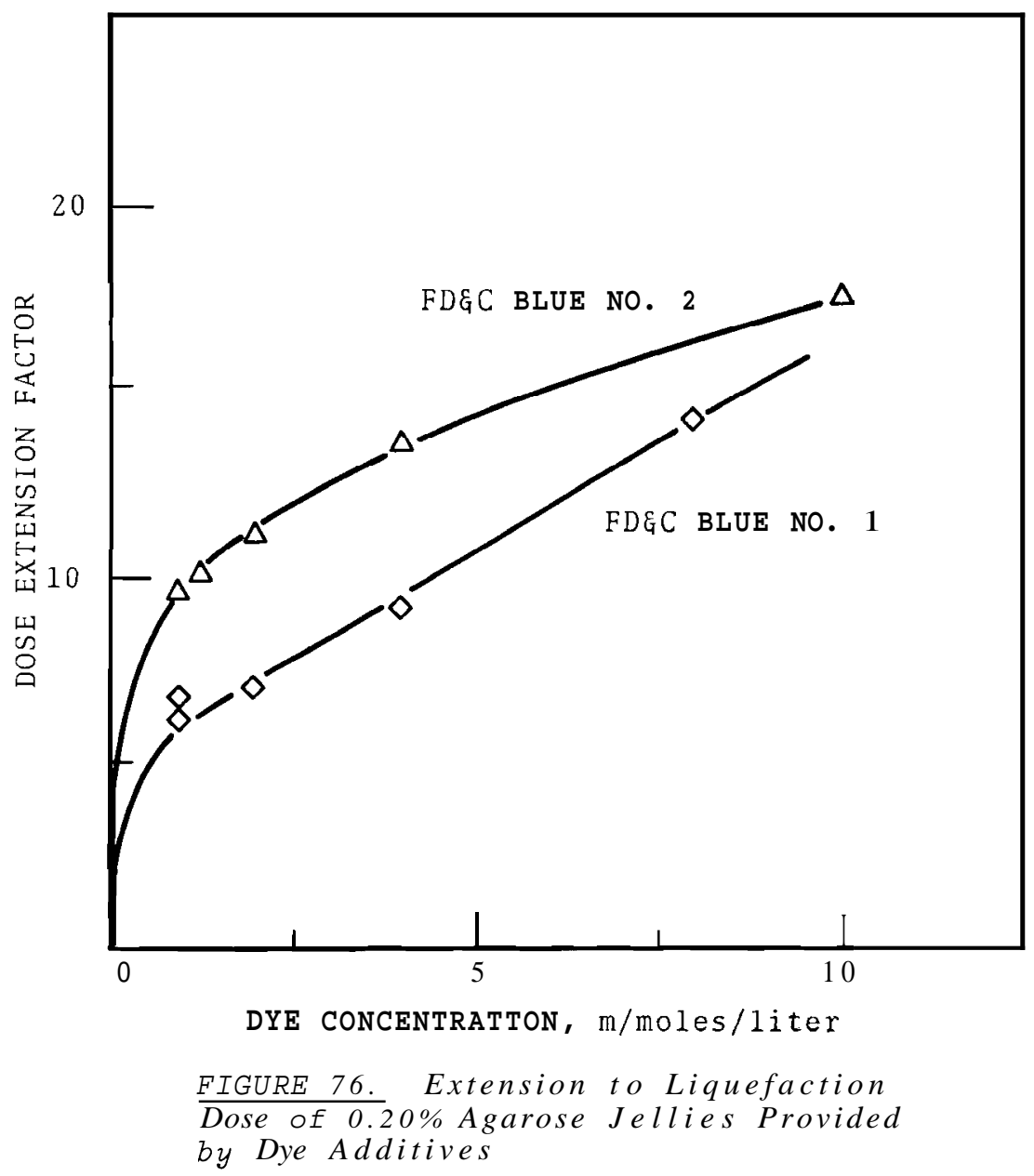

significant extensions to the radiation gether with their low toxicity to humans stabilities of the jellies even at millimakes them good candidates for further molar concentrations. This feature toresearch on the chemical protection of living tissue. 
RADIOLOGICAL CHEMISTRY

ALPHA-EMITTERS IN THE COLUMBia RIVER-C. D. Jennings* and T. M. Beasley

Long-lived actinides would represent a potential hazard if some means of concentrating them in the environment were to exist. Although their specific activities are low, their long half-lives dictate an extended residence in the environment. It is of interest, therefore, to determine which actinides are present in the Columbia River and at what concentration levels they exist there.

The identification of the alphaemitting isotope measured was accomplished both by large area alpharay spectrometry ${ }^{(85)}$ and by specific radiochemical separation techniques. Since large area alpha spectrometry requires a minimum of source material to obtain good resolution and since the levels of the alpha activity were expected to be small, it was necessary to concentrate the actinides from the ashed residues of relatively large biological samples. Following dissolution of the latter in concentrated nitric and perchloric acids, the actinides were coprecipitated on $\mathrm{Fe}(\mathrm{OH})_{3}$, the precipitate was isolated and dissolved in dilute nitric acid, and the actinides were extracted from a heavily salted $\mathrm{Al}\left(\mathrm{NO}_{3}\right)_{3}$ solution into $20 \%$ solution of alamine-336 in xylene.

A 11 tetravalent and hexavalent actinides are extracted essentially quantitatively under these conditions. The actinides were then removed from

*AEC Richland Graduate Felzow, June-September, 1965 the organic layer by stripping the solution with $1 \mathrm{M}^{\mathrm{HClO}} 4$ and the actinides again concentrated by coprecipitation on $\mathrm{Fe}(\mathrm{OH})_{3}$. This latter precipitate was then dissolved in 1M $\mathrm{CH}_{3} \mathrm{COOH}$, and the resulting solution aspirated onto aluminized cellulose acetate sheets for alpha-energy analysis. It must be pointed out that the isotopes of radium and the trivalent actinides are not carried through this particular separation.

Water samples were made acidic by the addition of nitric acid, reduced in volume by boiling, and the actinides concentrated by coprecipitation on $\mathrm{Fe}(\mathrm{OH})_{3}$. This precipitate was then processed as described above.

Table IX contains the qualitative results obtained from alpha spectrometric analysis of the variety of

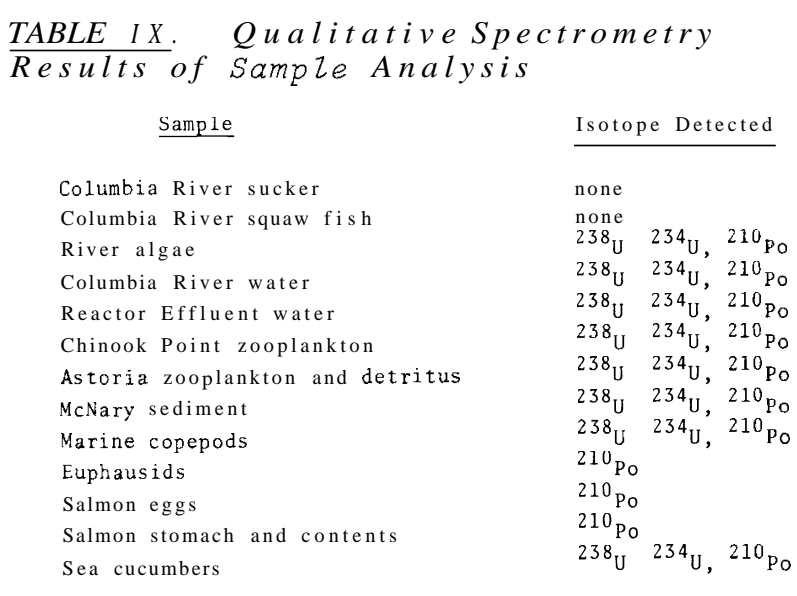

samples listed, while Table $X$ contains the quantitative results obtained in verifying both the identity of the 
TABLEX. Quantitative Results of Specific Radiochemical Analyses

Sample Size
10 liters
10 liters
15.51 iters
$2.0 \mathrm{~g}$
$28.0 \mathrm{~g}($ dry $)$
$3.5 \mathrm{~g}($ dry $)$
$69.0 \mathrm{~g}($ wet $)$
$70.7 \mathrm{~g}$ (wet)
$69.0 \mathrm{~g}$ (wet)

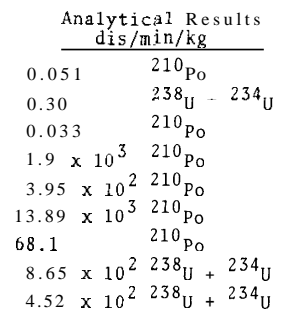

radionuclides detected and the quantities present. The only alpha emitters detected were ${ }^{234} \mathrm{U}, 238_{\mathrm{U}}$ and 210 Po. Cherry $(86)$ has measured the alpha radioactivity of plankton from the sea and reports that ${ }^{210}$ Po contributes a substantial portion of the alpha activity to these samples. A certain portion of the alpha activity of the plankton was unidentified (no spectrometry employed) and was assumed to be due to either uranium or radium.

The data of Tables IX and X suggest that indeed such activity could be due to uranium. The surprisingly high uranium content of sea cucumbers was verified by specific uranium analysis as was the high ${ }^{210}$ Po content of the salmon and sediment samples. (88)

The values of ${ }^{210}$ Po concentration for both reactor effluent water and river water are in general agreement with ${ }^{210} \mathrm{Po}$ and ${ }^{210} \mathrm{~Pb}$ concentration measured in ocean water (89) and other areas, ${ }^{(90)}$ although secular equilibrium between these two isotopes should not be assumed.

The data presented here and elsewhere $(91)$ would tend to substantiate the suggestion of Cherry (86) that the concentration of ${ }^{210} \mathrm{Po}$ (and possibly of ${ }^{210} \mathrm{~Pb}$ ) in the marine environment may be large. No artificially produced alpha-emitters were evidenced; if present they were below the detection limit of the instruments used. Alpha emitters were present only in low concentration in the Columbia River.

THE REDUCTION OF REACTOR EFFLUENT WATER RADIONUCLIDE CONCENTRATIONS BY THE ADDITION OF SODIUM SILICATE TO PROCESS WATER-D. E. Robertson

\begin{abstract}
Significant reductions in the concentrations of many reactor effluent water radionuclides by the addition of 10 to $20 \mathrm{ppm}$ of sodium silicate to process water was demonstrated. The experiment involved a halfplant evaluation at D-reactor which started in January of 196.5 and $r o n-$ tinued into 1966. The concentrations of the radionuclides $76 \mathrm{As}, 5 \mathrm{Cr}^{\mathrm{C}},{ }^{3} 2_{\mathrm{P}}$, $239 \mathrm{~Np}$, and $124 \mathrm{Sb}$ were reduced $i \mathrm{n}$ the effluent water from the test side to about 1/9.5, 1/7, 1/5, $1 / 3$ and $1 / 4$ respective $2 y$ relative to the control side of the reactor. The concentrations of $56 \mathrm{Mn}, 64 \mathrm{Cu}$ and $65 \mathrm{Zn}$ were essentially unchanged by the silicate treatment, while the concentrations of $24 \mathrm{Na}$ and $14{ }_{\mathrm{La}}$ land probably of the other rare earth radionuclides) were increased by about 25 to $35 \%$ and by a factor of 2 to 3 respectively. The behavior of $60 \mathrm{CO}$ and $46 \mathrm{SC}$ was more erratic.
\end{abstract}

A constant effort is being maintained at Hanford to reduce the amounts of radioactive material discharged to the Columbia River in reactor effluent 
water. (92) The radioisotopes and their interactions with the environment are continuously being monitored to determine the levels at communities which use Columbia River water for drinking purposes or for irrigation or which use its fish as food. (93)

The radionuclides of major interest are ${ }^{76} \mathrm{As},{ }^{32} \mathrm{P},{ }^{239} \mathrm{~Np},{ }^{51} \mathrm{Cr},{ }^{24} \mathrm{Na}$, ${ }^{64} \mathrm{Cu}$, and ${ }^{65} \mathrm{Zn}$.

The majority of the radionuclides present in reactor effluent water originate when their parent elements in process water become adsorbed on the corrosion film surface of the reactor process tubes and fuel element jackets and reside long enough to become neutron activated. Their radioactive daughters are then subsequently released to the effluent stream. (94) Minimizing the adsorption of these parent elements has reduced the concentrations of the radioactive daughters. Laboratory and in-reactor studies have been conducted to evaluate the effects of various chemical additives and coating materials as adsorption inhibitors. $(95-97)$ of the chemical additives tested in the laboratory, sodium silicate was, by far, the most effective adsorption inhibitor. The laboratory tests were followed by an in-reactor experiment in which sodium silicate, at 20 to $40 \mathrm{ppm}$ (as $\mathrm{SiO}_{2}$ ) was added to the process water flowing through a single reactor process tube. In that test the radionuclides ${ }^{32} \mathrm{P}$, ${ }^{51} \mathrm{Cr},{ }^{64} \mathrm{Cu},{ }^{76} \mathrm{As}$, and ${ }^{239} \mathrm{~Np}$ were reduced in the effluent water by factors to about $1 / 2,1 / 1.4,1 / 1.8$, $1 / 2.2$ and $1 / 3$, respectively, relative to their concentrations in the effluent water of a nearby control tube.
The concentration of ${ }^{65} \mathrm{Zn}$ and ${ }^{51} \mathrm{Cr}$ remained essentially unchanged, while those of ${ }^{24} \mathrm{Na}$ and ${ }^{31} \mathrm{Si}$ were increased by factors of 2 to 3 . The favorable results from this test prompted a production-scale experiment in which sodium silicate was added to the process water supplying one-half of a reactor while the other half, supplied with normal process water, was used as a control. This paper is a summary of the data obtained during this half-reactor test.

The half-plant silicate addition was conducted at the D Reactor. The "N" grade silicate (Philadelphia Quartz Company) was selected because of its higher purity and higher silica content $\left(\mathrm{SiO}_{2}\right.$ to $\mathrm{Na}_{2} \mathrm{O}$ ratio of 3.2:1) relative to other available sodium silicates. The sodium silicate was added (as a 1:5 dilution of the "N" silicate) to the process water supplying the test side of the reactor at a point following the water treatment process. Final pH adjustment was made in large storage tanks before pumping the water to the reactor. The addition was started on January 17, 1965 at a concentration of 5 ppm $\mathrm{SiO}_{2}$. The concentration was gradually increased to 10 to $20 \mathrm{ppm} \mathrm{SiO}_{2}$ over a period of 9 days. The concentration throughout the remainder of the test averaged about 12 to 15 ppm $\mathrm{SiO}_{2}$. During reactor shutdowns the silicate addition was continued but at a reduced concentration of 5 to $10 \mathrm{ppm} \mathrm{SiO}_{2}$. Daily samples of $500 \mathrm{ml}$ of effluent water from the test side and from the control side of the reactor were collected for radiochemical analysis from sampling lines continuously carrying a flow. The radionuclides ${ }^{24} \mathrm{Na},{ }^{32} \mathrm{P},{ }^{5}{ }^{\mathrm{Cr}} \mathrm{\text {, }}$ 
TABLE XI. Reduction Factors Obtained for Reactor Effluent Water Radionuclide Concentrations by the Addition of Sodium Silicate to Process Water

\begin{tabular}{|c|c|c|c|c|c|c|c|c|c|c|c|c|c|c|}
\hline$\underline{\text { Radionuclide }}$ & $\begin{array}{l}\text { Dec. } \\
(1964)\end{array}$ & ( ${ }_{(1965)}$ & Feb. & Mar. & Apr. & May ${ }^{(b}$ & June & $\mathrm{J} u 1 \mathrm{y}$ & Aug. & Sept. & Oct. & Nov. & Dec. & $\begin{array}{l}\text { Jan. } \\
(\underline{1966})\end{array}$ \\
\hline${ }^{24} \mathrm{Na}$ & 1.0 & 0.77 & 0.56 & 0.50 & 0.67 & 1.0 & 0.63 & 0.69 & 0.67 & 0.71 & 0.77 & 0.77 & 0.71 & 0.77 \\
\hline${ }^{32} \mathrm{p}$ & 0.77 & 0.26 & 0.70 & 1.5 & 1.7 & 2.4 & 1.6 & 3.2 & 2.6 & 2.8 & 2.5 & 1.5 & 2.6 & 3.2 \\
\hline${ }^{46} \mathrm{SC}$ & - & - & -- & - & 2.6 & 0.93 & 0.68 & 3.1 & 0.24 & -- & -- & -- & $\cdots$ & 12 \\
\hline${ }^{51} \mathrm{Cr}$ & 1.0 & 1.0 & 1.5 & 1.7 & 2.2 & 1.8 & 2.3 & 3.7 & 4.6 & 4.8 & 4.8 & 4.3 & 7.0 & 6.8 \\
\hline${ }^{56} \mathrm{Mn}$ & 1.0 & 0.87 & 0.56 & 0.59 & 0.77 & 0.67 & 0.71 & 0.91 & 0.80 & 0.74 & 0.63 & 0.80 & 0.80 & 0.83 \\
\hline${ }^{60} \mathrm{Co}$ & -- & - & -- & -- & 1.01 & 0.76 & 0.52 & 0.56 & 0.91 & - & - & - & - - & 0.75 \\
\hline${ }^{64} \mathrm{Cu}$ & 1.0 & 1.1 & 0.88 & 0.94 & 1.0 & 1.6 & 1.0 & 1.4 & 1.1 & 1.8 & - - & - - & -- & - - \\
\hline${ }^{65} \mathrm{Zn}$ & 1.0 & 0.83 & 0.77 & 0.77 & 1.0 & 0.83 & 1.0 & 1.0 & 1.0 & 0.90 & 1.0 & 0.90 & 1.4 & 1.9 \\
\hline${ }^{76} \mathrm{As}$ & 1.0 & 0.77 & 1.4 & 2.2 & 3.2 & 3.0 & 3.5 & 5.0 & 6.2 & 7.4 & 9.0 & 5.4 & 9.0 & 9.5 \\
\hline${ }^{124} \mathrm{Sb}$ & -- & - - & - - & - & 3.9 & 3.9 & 3.0 & 4.6 & 4.8 & - - & - - & - - & - - & 10 \\
\hline${ }^{239} \mathrm{~Np}$ & 1.0 & 0.90 & 1.7 & 2.3 & 4.0 & 2.5 & 3.3 & 4.4 & 3.7 & 4.0 & 3.4 & 3.4 & 5.2 & 5.2 \\
\hline${ }^{140} \mathrm{La}$ & -- & - - & - - & - - & - & - & - - & - & -- & 0.27 & 0.58 & -- & - & - \\
\hline
\end{tabular}

(a) Background data prior to addition

(b) Silicate was not added from May 11 to May 19, 1965

${ }^{56} \mathrm{Mn},{ }^{64} \mathrm{Cu},{ }^{65} \mathrm{Zn},{ }^{76} \mathrm{As}$, and ${ }^{239} \mathrm{~Np}$ were measured in the effluent water from both sides of the reactor by standard radiochemical and instrumental methods. The radionuclides ${ }^{46} \mathrm{Sc},{ }^{60} \mathrm{Co}$, ${ }^{124} \mathrm{Sb}$, and ${ }^{140} \mathrm{La}$, which are present in much lower concentrations, were periodically measured by directly counting the residue from evaporated effluent water samples using multidimensional gamma-ray spectrometry. (98) Dissolved silica was measured in the effluent from both sides of the reactor by a colorimetric procedure.

Background radionuclide measurements were made for several weeks prior to starting the addition and these showed that the concentrations of the radionuclides being studied were essentially equal in the effluent from both sides of the reactor. The silicate addition began on January 17,1965 , and continued into 1966. Thus, any seasonal trends due to water temperature, trace element concentrations in the river water, etc., could be observed. During the first several weeks of the addition (at 5 to $10 \mathrm{ppm} \mathrm{SiO}_{2}$ ) the sodium silicate caused a slight increase in most effluent water radionuclides and a large increase in the ${ }^{32} \mathrm{P}$ concentration (see Table XI). This was interpreted as a gradual sloughing-off of the old corrosion film which had the effluent water radionuclides incorporated into its structure. After the sloughing-off period a reduction in most radionuclides was observed, which gradually improved over a period of several months (see Table XI). After about 8 months of sodium silicate addition the reduction mechanism appeared to reach an equilibrium condition until the reactor was shut down for several weeks in early November for maintenance work. After startup the reductions in most radionuclides again were improved. As Table XI indicates, the 
maximum average monthly reductions obtained for ${ }^{32} \mathrm{P},{ }^{51} \mathrm{Cr},{ }^{76} \mathrm{As}$ and ${ }^{239} \mathrm{~Np}$ during the year were to $1 / 3.2,1 / 7$, $1 / 9.5$ and $1 / 5.2$, respectively, of the original values. The ${ }^{24} \mathrm{Na}$ and ${ }^{56} \mathrm{Mn}$ concentrations were increased 20 to $50 \%$ throughout most of the test. The concentrations of ${ }^{64} \mathrm{Cu}$ and ${ }^{65} \mathrm{Zn}$ were essentially unchanged. The behavior of the trace radionuclides ${ }^{46} \mathrm{Sc},{ }^{60} \mathrm{Co}$, ${ }^{124} \mathrm{Sb}$ and ${ }^{140}$ La was more difficult to interpret. These radionuclides are associated, to an extent, with particulate matter in the water and the variations of the ${ }^{46} \mathrm{Sc}$ and ${ }^{60}$ Co concentrations were rather extreme. However, the ${ }^{124} \mathrm{Sb}$ concentration was reduced to about $1 / 3$ to $1 / 5$. The ${ }^{140} \mathrm{La}$ (and probably the other less abundant rare earths) and ${ }^{60}$ Co were increased by factors of about 2 to 3 and 1.3 to 2 , respectively. The variations in the ${ }^{46}$ Sc concentration were so great that the effect of the silicate addition on its effluent concentration is still uncertain. Several samples showed the presence of ${ }^{59} \mathrm{Fe}$ and ${ }^{134} \mathrm{Cs}$ and their concentrations in the silicate-treated effluent water were about $1 / 6$ of those in the normal effluent water.

The mechanism by which sodium silicate reduces reactor effluent water radionuclide concentrations is not fully understood. However, its role as an adsorption inhibitor is clearly part of the mechanism. Silicates, whether present as ions or as micelles, tend to deposit on metal surfaces due to their negative charge, forming a very thin, inert surface. Definite alum inum silicate compounds have been reported to exist under conditions similar to those established in this test. Analysis, by emission spectroscopy, of the corrosion film on rear nozzle Van Stone flange inserts (perforated aluminum cylinders) from both sides of the reactor showed a silicon concentration about five times higher in the film of the test side. X-ray diffraction measurements showed this film to consist mainly of an amorphous material, probably amorphous silica, as reported in other similar investigations. Apparently, silicates react with the hydrous aluminum oxide surface to form the protective film. Since essentially a 11 of the radionuclides the concentrations of which were reduced by the silicate treatment were in the form of anions, it was suspected that the silicated film exhibited a slightly negative charge, thereby inhibiting the adsorption of additional anions from the process water onto the film. Zeta potential measurements of fresh suspensions of the corrosion films confirmed this to be true. The silicated film had a zeta potential of -17 to $-21 \mathrm{mV}$, while the unsilicated film was essentially at zero mV. There is, however, no large corresponding increase in the cation radionuclides in the silicatetreated effluent. The silicated corrosion film is so inert compared with the highly active normal corrosion film, that a preferential adsorption of cations is not obtained.

One of the problems encountered during this test was an abnormal filming in the fringe process tubes supplied by silicate-treated process water and a consequent hydraulic pressure increase. This occurred only when the inlet water temperature was below $10{ }^{\circ} \mathrm{C}$ 
and only in those fringe tubes where the water temperature was not significantly increased by the heat generated in the reactor. (99) Obviously, these conditions created a change in the physical and/or chemical nature of the sodium silicate which allowed greater deposition to occur. When the water temperature increased during the summer months this differential filming effect ceased.

Another problem which occurred during the test was an increase in the radiation levels on the rear face of the test side; this averaged 2 to 4 times higher than on the control side, thus increasing the problem of exposure to personnel working on the rear face.

This radiation decayed with approximately a 12 to $15 \mathrm{hr}$ half-life and both sides appeared to decay at nearly the same rate. Corrosion film deposited on the rear nozzle Van Stone flange inserts from both sides of the reactor was radiochemically analyzed to determine the cause of the higher radiation readings on the test side. It appeared that rare earth radionuclides. notably ${ }^{140} \mathrm{La}$ and ${ }^{153} \mathrm{Sm}$ deposit on the downstream side of the reactor piping. These radionuclides were in greater abundance in the corrosion film from the test side by a factor of 2 to 3 . When the silicated-film preferentially adsorbs the rare earth radionuclides is, at present, unexplained.

Sodium silicate is an effective adsorption inhibitor for parents of many of the important effluent water radionuclides. The factors for and against using sodium silicate treated process water $\mathbf{t} \sim$ reduce radionuclide concentrations in reactor effluent water must be carefully weighed before a decision to use it on a full scale basis can be made. The radionuclides ${ }^{76}$ As, ${ }^{239} \mathrm{~Np}$, and ${ }^{51} \mathrm{Cr}$ were reduced to about $1 / 9.5$, $1 / 5.2$, and $1 / 7$, respectively, of their original values. These radionuclides are important because they contribute over two-thirds of the gastrointestinal dose to the residents of Richland,

Pasco and Kennewick who use Columbia River water for drinking purposes. The radionuclide ${ }^{32} \mathrm{P}$, which is concentrated by whitefish and waterfowl inhabiting the Columbia River, and thus, consumed by people, was reduced in concentration to about $1 / 3$ by the silicate treatment. The concentrations of ${ }^{124} \mathrm{Sb}$ and ${ }^{64} \mathrm{Cu}$ were reduced to about $1 / 4$ and $1 / 1.3$, respectively. The ultimate goal of these reactor effluent water studies is to reduce the radionuclide concentrations in the Columbia River. If sodium silicate were used on a full-scale (5 reactors) basis, the river concentrations of the above radionuclides could then be expected to be reduced by the above amounts. The only increases of significance found were for ${ }^{24} \mathrm{Na}(25$ to $35 \%)$ and the trace amounts of rare earth radionuclides (a factor of 2 to 3 ). The filming in cold tubes and the rear face exposure problems are unfavorable side effects; these might be eliminated or reduced when better understanding of the mechanisms involved is obtained.

Throughout the test there existed some cross-mixing of water from both sides of the reactor. It has been estimated that this cross-mixing amounted to about $15 \%$ of the total reactor ef$\mathrm{flu}$ ent being interchanged. The efficiency of this test could then be expected to improve by such an amount in full-scale practice. 
THE EFFECT OF REDUCED ALUM ADDITION ON THE REMOVAL OF ${ }^{75}$ AS AT THE KW-REACTOR WATER TREATMENT PLANT-W. A. H a Il e r

For several months experiments were carred out at the KW- and KE-Reactors to evaluate the effects of reducing the concentration of alum in the water treatment process. Of particular interest were changes in the concentrations of radioisotopes introduced into the Columbia River. Reactor effluent samples were routinely taken and analyzed for their radionuclide concentrations.

During the month of December the KE-Reactor water treatment plant used a 16 ppm alum addition while the KW-Reactor water treatment plant used $6.5 \mathrm{ppm}$. Analyses of the reactor effluent waters showed that the ${ }^{76}$ As concentration in the KW effluent water was 5 to 6 times that of KE. Since the ${ }^{75}$ As $(n, \gamma){ }^{76}$ As reaction is responsible for ${ }^{76}$ As production, one explanation of the observed increase of the latter isotope is that a decreased alum addition decreased the removal of the parent isotope ${ }^{75}$ As at the water treatment plant.

Neutron activation analysis was used to determine the efficiencies for the removal of the parent isotope ${ }^{75}$ As at the water treatment plant.

Ten milliliter samples of raw and alum-treated water were collected at the KW and KE plants and these were neutron activated for $24 \mathrm{hr}$. After a three day cooling period, the arsenic was chemically separated from the irradiated samples using the ben- zene $\mathrm{HCl}$-HI extraction method. (100) The ${ }^{76}$ As was then counted with a 5 in. NaI crystal; the observed activity is shown in Table XII,

TABLE XII. Activation of Raw and Treated Water from the $K$-Reactors Water Treatment Plants

\begin{tabular}{|c|c|c|c|c|}
\hline $\begin{array}{l}\text { Collection } \\
\text { Date }\end{array}$ & $\begin{array}{l}\text { Water Treatment } \\
\text { Plant } \\
\end{array}$ & $\begin{array}{l}\text { Addition, } \\
\text { ppm }\end{array}$ & $\begin{array}{l}76 \text { As }(a) \\
\text { Counts } \\
\end{array}$ & $\begin{array}{l}\text { Efficiency } f F^{r} \\
\text { Removal of } \\
\frac{\&}{\sigma}(b) \\
\end{array}$ \\
\hline $12 / 1 / 65$ & KW (Treated) & 6.5 & 17,006 & 51.5 \\
\hline $12 / 1 / 65$ & KE (Treated) & 16 & 4,164 & 88.1 \\
\hline $\begin{array}{l}12 / 1 / 65 \\
12 / 6 / 65\end{array}$ & $\begin{array}{l}\text { KW (Raw Water) } \\
\text { KW (Treated) }\end{array}$ & $\begin{array}{l}0 \\
6.5\end{array}$ & $\begin{array}{l}35,021 \\
17,181\end{array}$ & 51.0 \\
\hline $12 / 6 / 65$ & KE (Treated) & 16 & 4,152 & 88.2 \\
\hline
\end{tabular}

(a) 75 As input concentration may be measured by the ${ }^{76}$ As output concentration as generated by the neutron activation procedure.

(b) Efficiency of removal was calcylated by comparing the raw water As content versus the treated water ${ }^{76}$ As content produced by neutron activation.

Table XII clearly shows that the efficiency of removal of $75^{A s}$ at a reactor water treatment plant decreases with a low alum addition. Table XII and the analytical laboratory analyses also show that there is a direct relationship between the ${ }^{75}$ As input into the reactor and the ${ }^{76}$ As output. The ${ }^{76}$ As output of KW is 5 to 6 times higher than that of KE, while the corresponding input of parent ${ }^{75}$ As into these reactors differs by a factor of 4 , being higher at KW at least for the period of time concerned. 
ION EXCHANGE STUDIES OF THE CHEMICAL FORM OF RADIONUCLIDES IN COLUMBIA RIVER WATER AND REACTOR EFFLUENT- JackL. NeISOn

Ion exchange studies with reactor effluent and Columbia River water from Hanford to Vancouver, Washington indicate that $65 \mathrm{Zn}, 60 \mathrm{Co}$, and Mh are Zargezy cationic while ${ }^{51} \mathrm{Cr}$ is mostly anionic. 124Sb is anionic in reactor effluent and is changed to an uncharged form as it moves down the river.

One of the first steps that is necessary in a study of the mechanisms involved in reactions of radionuclides with river sediments is to determine the chemical and physical forms of the nuclides in the river. Experiments have been conducted in which samples of river water were filtered through $0.3 \mu$ membrane filters and then passed through ion exchange resin beds. Ions found in the effluent of cation exchange resin columns were considered to be either anions or uncharged forms; those in anion resin effluent were cations or uncharged; and those passing through the two resins in series were uncharged species. The experiments were run immediately after sampling the reactor effluent and at four sites down the Columbia River to Vancouver, Washington. Series of experiments were repeated in March, June, and September to detect any differences due to temperatures, light, and river discharge.

The results of the experiment are given in Table XIII. Since there appeared to be no significant differences attributable to season, the data for the three dates were averaged. It can be seen that the percentage of each radionuclide except ${ }^{124} \mathrm{Sb}$ found to be in particulate form increased as reactor effluent was mixed with river water and for some distance downstream but then seemed to reach a dynamic equilibrium.
An equilibrium level was reached for most of the nuclides about at McNary Dam but, for ${ }^{60}$ Co, was reached at the sampling point in the 300 Area of the Hanford project. This equilibrium condition is thought to be a state in which settling out and deposition of suspended sediment is occurring at about the same rate as additional uptake of nuclides by suspended sediment. For ${ }^{124} \mathrm{Sb}$, it would appear that the fraction associated with particulates gradually settled out to the river bottom and that little or no additional uptake by particles occurred.

TABLE XUU. Forms of Radionuclides River Water at Various Locations

\begin{tabular}{|c|c|c|c|c|c|}
\hline \multirow[b]{2}{*}{ Radionuclide } & \multirow[b]{2}{*}{ Location } & Percent & \multicolumn{3}{|c|}{ Percent in Solution } \\
\hline & & Particulate & Cationic & Anionic & Uncharged \\
\hline \multirow{3}{*}{${ }^{65} \mathrm{zn}_{\mathrm{n}}$} & R.E. (a) & 16 & 100 & 0 & 0 \\
\hline & $\begin{array}{l}300 \text { Area } \\
\text { McNary }\end{array}$ & $\begin{array}{l}31 \\
77\end{array}$ & $\begin{array}{l}90 \\
98\end{array}$ & $\begin{array}{l}1 \\
1\end{array}$ & $\begin{array}{l}3 \\
1\end{array}$ \\
\hline & $\begin{array}{l}\text { The Dalles } \\
\text { Vancourer }\end{array}$ & $\begin{array}{l}84 \\
80\end{array}$ & $\begin{array}{l}96 \\
83\end{array}$ & $2^{2}$ & $2_{5}^{2}$ \\
\hline \multirow{4}{*}{${ }^{51} \mathrm{Cr}$} & R.E. & 2 & 3 & 12 & 1 \\
\hline & 300 Area & 3 & 3 & 96 & 1 \\
\hline & McNary & 7 & 3 & 93 & 3 \\
\hline & $\begin{array}{l}\text { The Dailes } \\
\text { Vancouver }\end{array}$ & 10 & 1 & 99 & 0 \\
\hline \multirow{4}{*}{${ }^{60} \mathrm{Co}$} & R.E. & 5 & 98 & & 0 \\
\hline & $\begin{array}{l}\text { 3.0 Area } \\
\text { McNary }\end{array}$ & 63 & 89 & $\frac{9}{4}$ & $5^{2}$ \\
\hline & The Da11es & 64 & 57 & 30 & 13 \\
\hline & Vancouver & 68 & 81 & 3 & 16 \\
\hline \multirow{4}{*}{${ }^{46} \mathrm{Sc}$} & R.E. & $\begin{array}{l}68 \\
77\end{array}$ & 34 & $\begin{array}{l}62 \\
83 \\
\end{array}$ & $\begin{array}{l}5 \\
4 \\
\end{array}$ \\
\hline & MeNary & 90 & 14 & 79 & 7 \\
\hline & The Dalles & 88 & 28 & 56 & 16 \\
\hline & Vancouver & 89 & 4 & 79 & 17 \\
\hline \multirow{4}{*}{${ }^{54} \mathrm{Mn}$} & R.E. & 3 & 97 & ${ }_{8}^{2}$ & $\begin{array}{r}0 \\
16\end{array}$ \\
\hline & MeNary & 70 & $\begin{array}{l}95 \\
902\end{array}$ & $\frac{2}{2}$ & 3 \\
\hline & Vancouver & 70 & 48 & 43 & 10 \\
\hline & R.E. & 9 & 0 & 100 & 0 \\
\hline \multirow{3}{*}{${ }^{124} \mathrm{Sb}$} & 300 Area & 5 & 1 & 42 & 57 \\
\hline & $\begin{array}{l}\text { McNary } \\
\text { The Dalles }\end{array}$ & $\frac{2}{2}$ & $\begin{array}{l}0 \\
2\end{array}$ & $\begin{array}{l}22 \\
24\end{array}$ & $\begin{array}{l}78 \\
74\end{array}$ \\
\hline & Vancouver & 1 & 0 & 16 & 84 \\
\hline
\end{tabular}

(a) Reactor Effluent 
As expected, ${ }^{65} \mathrm{Zn},{ }^{60} \mathrm{Co}$, and ${ }^{54} \mathrm{Mn}$ were, and remained, mostly cationic while ${ }^{51} \mathrm{Cr}$ was anionic. ${ }^{46} \mathrm{Sc}$ had a surprisingly large fraction in the anion form plus some uncharged species. These, as well as the possible small amounts of uncharged ${ }^{60} \mathrm{Co}$ and ${ }^{65} \mathrm{Zn}$ are probably species complexed by biota or organic derivatives of biota. The ${ }^{124} \mathrm{Sb}$ was all anionic in reactor effluent but was largely changed to an uncharged form as it moved down the river.

These data agree well with depletion data (101) which indicate that ${ }^{51} \mathrm{Cr}$ and ${ }^{124} \mathrm{Sb}$ move down the river with little depletion while ${ }^{65} \mathrm{Zn},{ }^{46} \mathrm{Sc},{ }^{60} \mathrm{Co}$, and ${ }^{54} \mathrm{Mn}$ have a higher natural depletion from the river water. It will be of interest and importance to determine some of the chemical species and how they are taken up and held by sediments

DETERMINATION OF SEASONAL VARIATIONS IN SEDIMENT DEPOSITION RATES IN MCNARY RESERVOIR OF THE COLUMBIA RIVER- JackL. NeISON

During 1964 and early 1965, a series of bottom sediment cores was taken from an area about 1000 yards upstream from the McNary Dam on the Columbia River under $50 \mathrm{ft}$ of water. The sample site is an area of quiescent water not in direct line with spillways and turbine intakes of the dam. The coring device used was a $15 \mathrm{~cm}$ diam free-fall apparatus. (102) Cores were frozen, sliced in 1 to 5 am depth sections, dried, and analyzed with a multidimensional gamma ray spectrometer. (103)

Good data were obtained for ${ }^{65} \mathrm{Zn}$, ${ }^{60} \mathrm{Co}$, and ${ }^{46} \mathrm{Sc}$ and these are plotted with depth on semilog plots in Figures 77 through 82. Some less precise data were also obtained for ${ }^{54} \mathrm{Mn},{ }^{58} \mathrm{Co},{ }^{106} \mathrm{Ru}$, ${ }^{95} \mathrm{Zr}-{ }^{95} \mathrm{Nb},{ }^{59} \mathrm{Fe}$, and ${ }^{137} \mathrm{Cs}$, and these followed a pattern similar to that in the graphs shown.

The pattern of changes in radioactivity with depth can perhaps best be seen in the ${ }^{65}$ Zn curves. As expected from radioactive decay, there is a fairly good straight line decrease of ${ }^{65}$ Zn with depth with some notable exceptions, especially near the surface.

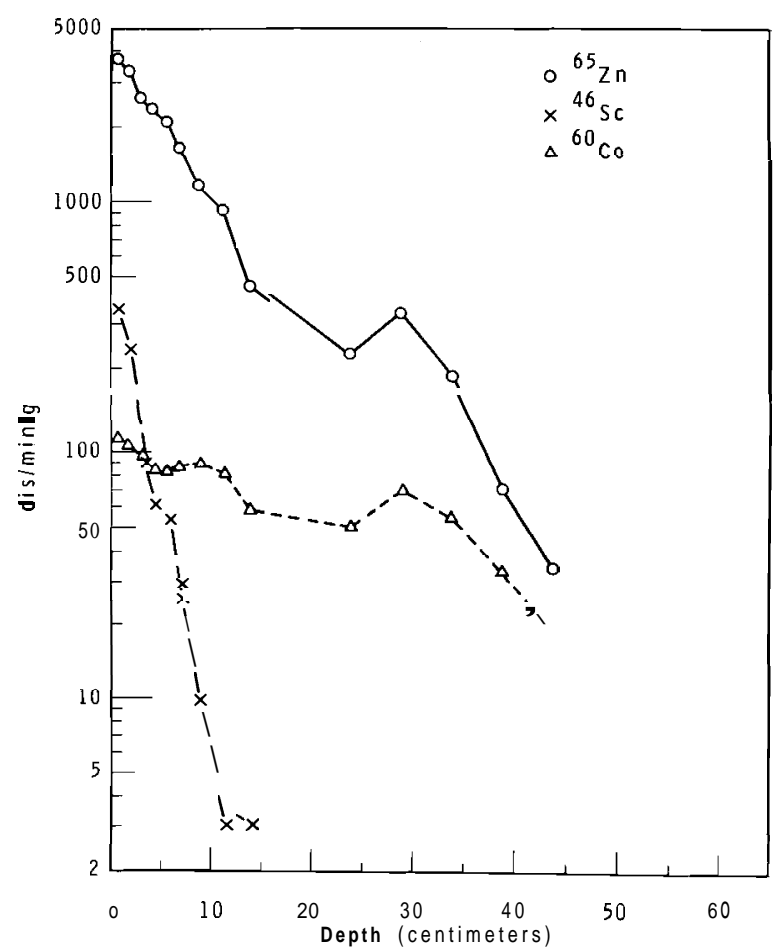

FIGURE 77. Variation of ${ }^{65} \mathrm{Zn},{ }^{60} \mathrm{Co}$, and ${ }^{46}$ Se with Depth in 4/27/64 MeNary Core

The major scouring of sediment in the river occurred between the $5 / 5 / 64$ core (Figure 78) and the 6/23/64 core (Figure 79). The two cores taken before the scouring period (Figures 77 and 78) 


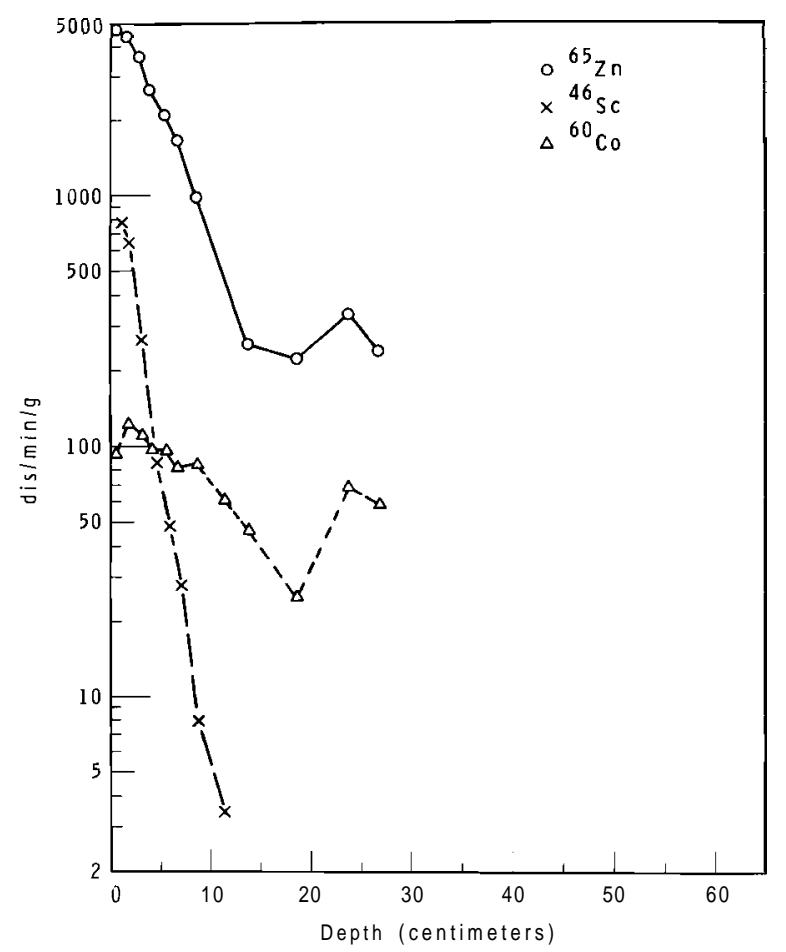

FIGURE 78. Variation of ${ }^{65} \mathrm{Zn},{ }^{60} \mathrm{Co}$, and ${ }^{46}$ Sc with Depth in 5/5/64 MeNary Core

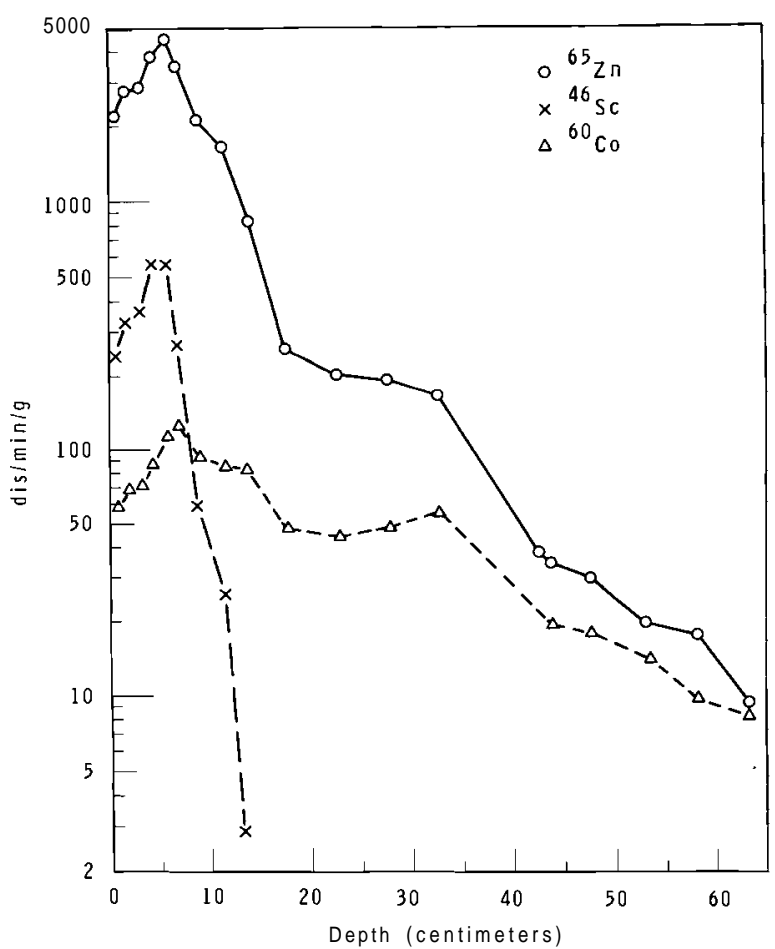

FIGURE 79. Variation of ${ }^{65} \mathrm{zn},{ }^{60}$ Co, and ${ }^{46}$ SC with Depth in 6/23/64 MeNary Core show relatively linear decreases in activity with depth. Immediately afterr the major scouring period $(6 / 23 / 64$, Figure 79), there was an increase in activity with depth for about the top $8 \mathrm{~cm}$, and then the expected decrease begins. The cores taken through the rest of the season showed patterns grading steadily between these two extremes.

I t would appear that the sampling site was an area in which sediment was being steadily deposited, even during the spring when other parts of the river bottom were being scoured by high water. The high water and large sediment load evidently resulted in a relatively rapid deposition of about $8 \mathrm{~cm}$ of low activity sediments during the spring freshet. This may be somewhat more

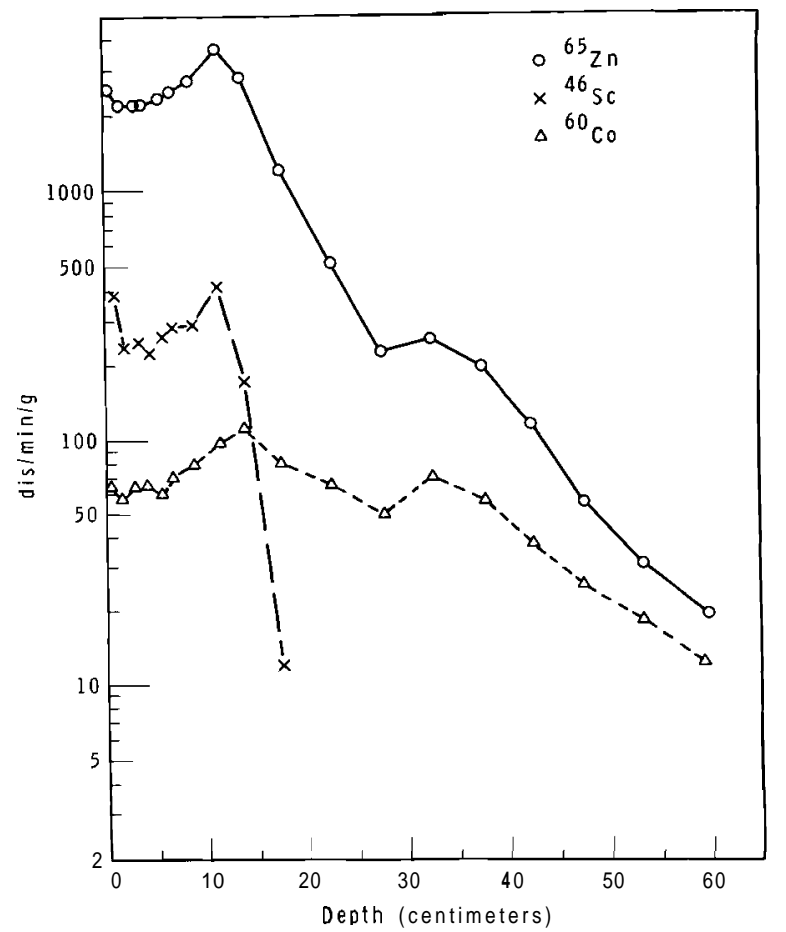

FIGURE 80. Variation of ${ }^{65} \mathrm{Zn},{ }^{60} \mathrm{Co}$, and ${ }^{46}$ Se with Depth in 7/17/64 MeNary Core 


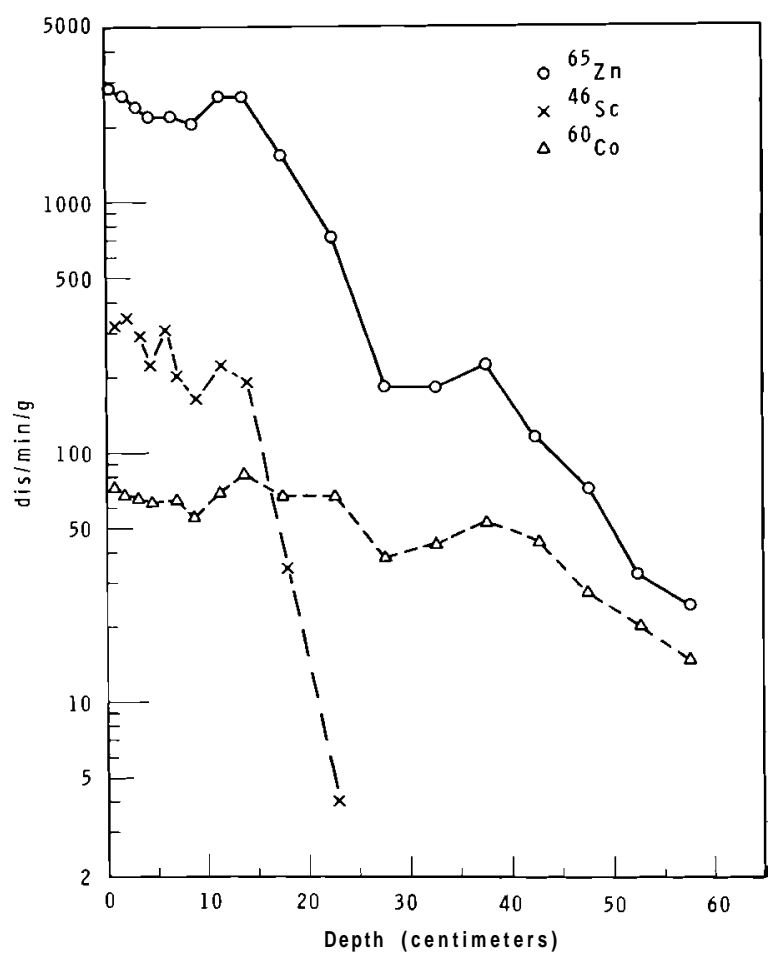

FIGURE 81. Variation of ${ }^{65} \mathrm{Zn},{ }^{60} \mathrm{Co}$, and ${ }^{46}$ SC with Depth in 8/24/64 MeNary Core

than would be deposited during the average freshet, because the 1964 spring water volume and suspended sediment levels were unusually high. A part of the deposited material was undoubtedly sediment that was scoured from areas of the river between Hanford and McNary. Indications of this are shown by a slightly sharper dip in ${ }^{46}$ Sc activity $\left(84\right.$ d) than ${ }^{60}$ Co activity $(5.3$ yr) in the top 8 am of sediment.

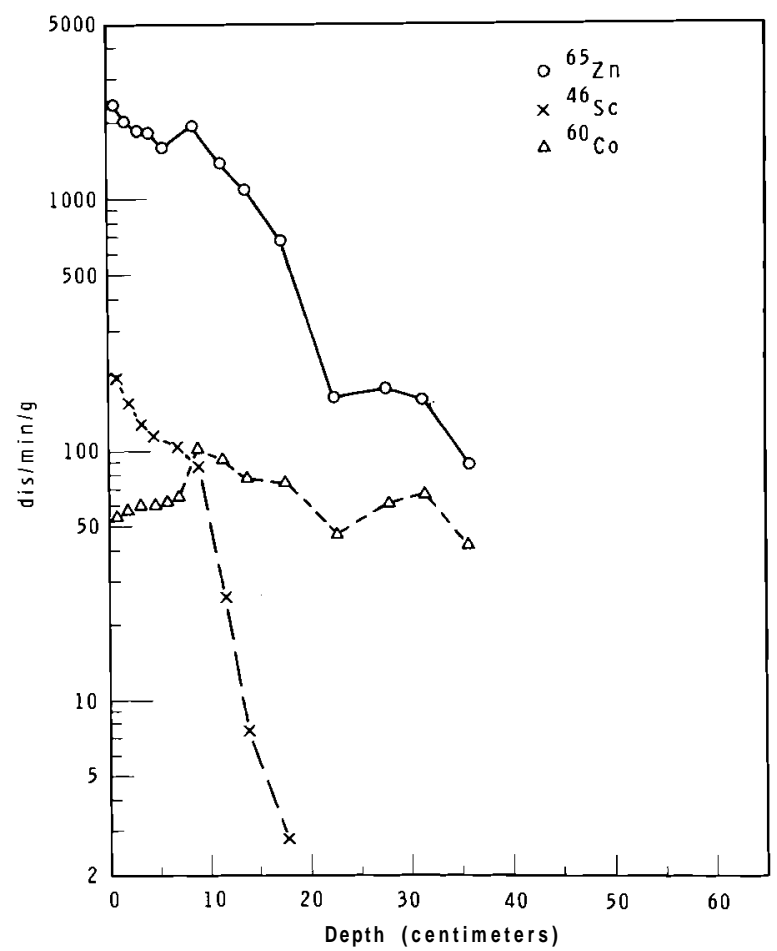

FIGURE 82. Variation of ${ }^{65} \mathrm{Zn},{ }^{60} \mathrm{Co}$, and ${ }^{46}$ Sc with Depth in $1 / 8 / 65$ McNary Core

In comparing these data with scouring data previously reported, (104) the situation appears to be one of slow sediment deposition over large areas of the river bottom during the July to April period. Then during the high water period of May and June, a large part of the deposited sediment from the upriver medium to high current areas is scoured and carried downriver where a part of it redeposits in low current areas such as the sampling area described in this report.

\section{THE USE OF ${ }^{65}$ Zn $/{ }^{60}$ CO RATIOS TO DETERMINE AGE AND DEPOSITION RATE OF}

\section{RIVER BOTTOM SEDIMENTS-Jack L. Nelson and J. M. Nielsen}

Radionuclide ratios have been used for many years to determine ages and deposition rates of ocean bottom sediments. (105) It is also possible under some circumstances to determine in the same way the age and deposition rate of river bottom or reservoir sediments but on a much shorter time scale than 
i s usually used in the oceans. (106)

The method can be applied only to areas of a river where net accumulation of sediment is occurring and is most useful where constant deposition is going on. Another requirement is that the ratio of input radionuclides be relatively constant. These conditions are met reasonably well for the past several years in portions of the Colimbia River below the Hanford Plant. A semilog plot of ${ }^{65} \mathrm{Zn} /{ }^{60}$ Co concentration versus sediment depth should result in a straight line decrease due to the difference in half-lives of the two nuclides. This assumes that radioactive decay is the sole or principal change occurring and that deposition is somewhat constant. Figures 83,84 , and 85 show such plots for bottom cores taken at three dates about

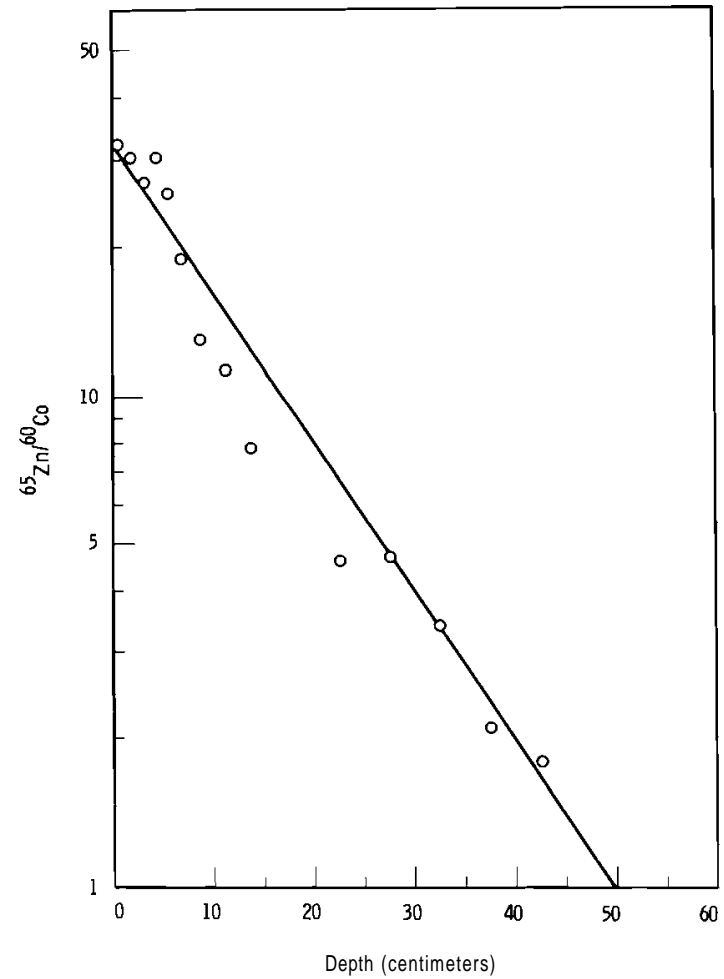

FIGURE 83. Least Squares Plot of ${ }^{65} \mathrm{Zn/}$ ${ }^{60}$ Co Ratio Versus Depth in Sediment Core from McNary Reservoir, 4/27/64

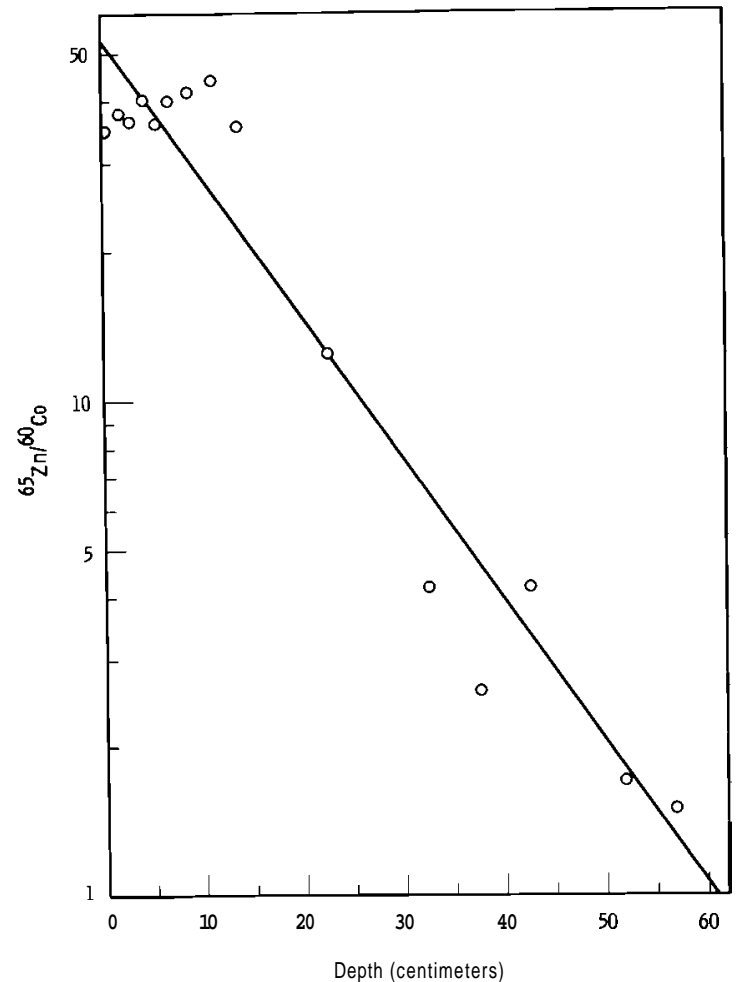

FIGURE 84. Least Squares Plot of ${ }^{65} \mathrm{Zn} /$ ${ }^{60}$ Co Ratio Versus Depth in Sediment Core from McNary Reservoir, 6/23/64

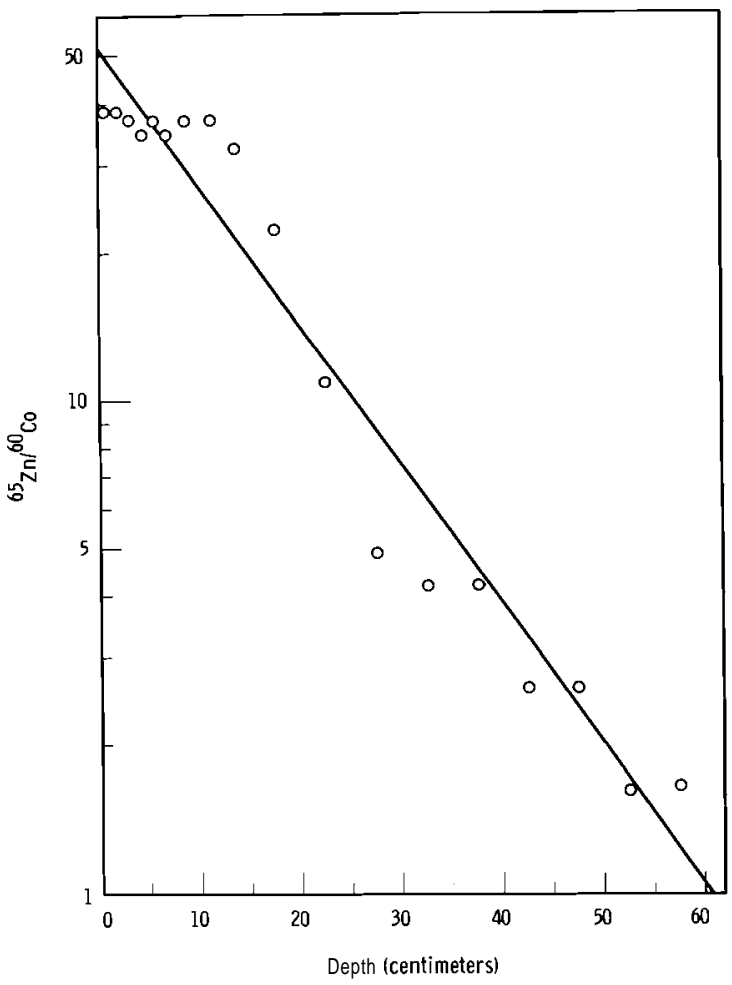

FIGURE 85. Least Squares Plot of ${ }^{65} \mathrm{Zn} /$ ${ }^{60}$ Co Ratio Versus Depth in Sediment Core from McNary Reservoir, 8/24/64 
1000 yards upstream from McNary Dam on the Columbia River. These are some of the same cores for which radionuclide data are given in an accompanying section of this report. (107)

The points shown are actual data points of ${ }^{65} \mathrm{Zn} /{ }^{60}$ Co ratios at various depths while the line is a calculated least squares fit line with intercepts evaluated at zero depth and a ${ }^{65} \mathrm{zn} /{ }^{60} \mathrm{Co}$ ratio of one. The intercept at zero depth should be a best value for the 65 Zn $/{ }^{60}$ Co ratio originally sorbed or deposited at the sediment surface. The slope of the line can be used to calculate an average deposition rate per unit of time using the 245 day half-life of ${ }^{65} \mathrm{Zn}$ and the $5.3 \mathrm{yr}$ halflife of ${ }^{60}$ Co. The surface ratios and average deposition rates for these and some other cores taken at the same location are given in Table XIV.

The ${ }^{65} \mathrm{Zn} /{ }^{60} \mathrm{Co}$ ratios found at the surface agree well with those in Columbia River water and suspended sediment at Pasco, Washington, about 36 miles upstream. (101) Analytical data on Hanford reactor effluent indicate that the ${ }^{65} \mathrm{Zn} /{ }^{60}$ Co ratio added to the river fluctuates but has not changed appreciably over the approximately 4 years covered by the plots. The average deposition rate of about $12.5 \mathrm{~cm} / \mathrm{yr}$ is high but consistent with other evidence. It should be emphasized that this is for one particular sampling area and is not representative of the whole McNary Reservoir. Sediment dep- . osition rates vary broadly with the current and other factors. They also vary somewhat with the season as can be seen in Figures 83, 84, and 85. Figure 84 shows a section in the top 8 to 10 cm where relatively rapid deposition has occurred during the recent spring freshet. The calculated line, then, is an average of all seasons of several years.

Attempts have been made to apply this dating method to cores in other areas of the Columbia River. Although there are several other areas of the river where a reasonably good straight line plot is obtained, parts of the river bottom are not applicable to this treatment because sediment is not being regularly deposited there.

TABLE XIV. ${ }^{65} \mathrm{Zn} /{ }^{60}$ Co Ratios at the Surface and Average Sediment Deposition

Rates for One Sampling Site in the McNary Reservoir of the Columbia River

\section{Core Date}

$4 / 27 / 64$

$5 / 5 / 64$

$6 / 23 / 64$ - No. 1

$6 / 23 / 64$ - No. 2

$7 / 17 / 64$

$8 / 24 / 64$

$1 / 8 / 65$

$$
\begin{array}{r}
{ }^{65} \mathrm{Zn} /{ }^{60} \text { Co Ratio } \\
\text { at Surface }
\end{array}
$$

31.9

37.0

53.8

35.2

47.3

51.3

43.5
Average

Deposition Rate, $\mathrm{cm} / \mathrm{yr}$

12.7

10.2

13.7

15.0

11.7

14.0

9.9

Overall Average 
A METHOD FOR DETERMINATION OF ${ }^{32} \mathrm{P}$ AND OTHER RADIONUCLIDES ON FILTERS CONTAINING SUSPENDED RIVER SEDIMENTS-Jack L. Nelson and W. B. Silker

\begin{abstract}
I $t$ is sometimes desired to separate and determine ${ }^{32}$ P associated with small amounts of sediment on membrane filters and subsequently to be able to determine other radionuclides in the same sample. A procedure found satisfactory is described below.
\end{abstract}

Quarter up to ten 5.5 in. diam filters or the equivalent by tearing or cutting, and place them in a $600 \mathrm{ml}$ beaker. Add 100 to $150 \mathrm{ml}$ of $50 \% \mathrm{HNO}_{3}$, $20 \mathrm{ml}$ of $30 \% \mathrm{H}_{2} \mathrm{O}_{2}$, and a few boiling beads. Cover the beaker with a watch glass and let it stand for $20 \mathrm{~min}$.

Place on a hot plate and begin heating on low heat. After it has been observed that the filters are beginning to disintegrate, turn the heat up to high. Before the sample goes to dryness, remove the beaker from the hot plate and add an additional $150 \mathrm{ml}^{\text {of }} 50 \% \mathrm{HNO}_{3}$ and $20 \mathrm{ml}$ of $30 \% \mathrm{H}_{2} \mathrm{O}_{2}$. Boil on high heat to near dryness and then decrease heat to low. Low heat avoids spattering while going to dryness. If charring appears, the oxidation is not complete and it is necessary to make additional $50 \% \mathrm{HNO}_{3}$ and $30 \% \mathrm{H}_{2} \mathrm{O}_{2}$ treatments.

Add $100 \mathrm{ml}$ of $1.2 \mathrm{M} \mathrm{HC1}$ to the beaker and heat nearly to boiling. Cool and transfer to a $500 \mathrm{ml}$ separatory funnel. Rinse with a small amount of $1.2 \mathrm{M} \mathrm{HC}$. Add a second $100 \mathrm{ml}$ of $1.2 \mathrm{M}$ HCl to the beaker and repeat the heating step.

Transfer this to the separatory funnel also. Police the beaker and rinse with 1. $2 \mathrm{M} \mathrm{HCl}$. Bring total volume in the separatory funnel to $250 \mathrm{ml}$ with $1.2 \mathrm{M}$ HC1.

Add $50 \mathrm{ml}$ of $10 \%$ ammonium molybdate solution and shake for $30 \mathrm{sec}$. Add $70 \mathrm{ml}$ of a solution of $13 \% 1$-butanol in anhydrous ether, invert once and vent to release pressure. Continue to invert and vent until pressure stops building up and then shake for $60 \mathrm{sec}$. Let the phases separate and drain the lower aqueous phase into the same 600 ml beaker in which the digestion was performed. Rinse the organic with 25 to $30 \mathrm{ml}$ of $1.2 \mathrm{M} \mathrm{HC1}$, venting as required. Shake for 30 sec, let phases separate, and add the aqueous to that already collected in the $600 \mathrm{ml}$ beaker. Transfer the organic to a 50 or $100 \mathrm{ml}$ beaker and let the ether evaporate overnight. Mount the butanol on a $B$ planchet and rinse the beaker with a very small amount of ethanol. Dry under an infrared lamp and count the planchet for ${ }^{32} \mathrm{P}$.

Evaporate the contents of the 600 ml beaker to dryness under a heat lamp When dry, place the beaker on a hot plate on medium or high heat until fuming stops. With small amounts of water, transfer the residue into a counting container for gamma spectroscopy. Police and rinse the beaker into the counting container. Count for gamma emitters.

The procedure has been tested for recovery of gamma emitters by first counting sectioned filters per se and comparing the data with gamma counts obtained following ${ }^{32} \mathrm{P}$ removal. The filters used were $0.30 \mu$ membrane fil ters used on about 6 gal of Columbia 
River water. The comparisons are shown in Table XV.

Although some correction may be desirable and occasional recover checks should be made, the recoveries found are quite good.

\begin{tabular}{|c|c|c|c|c|c|}
\hline \multirow[t]{2}{*}{ TABLE XV. } & \multicolumn{5}{|c|}{$\begin{array}{l}\text { Recovery of Gamma Emitters } \\
32_{P} \text { Removal Procedure }\end{array}$} \\
\hline & $60_{\mathrm{CO}}$ & ${ }^{46}{ }_{S c}$ & $65_{\mathrm{zn}}$ & ${ }^{54} \mathrm{Mn}$ & $\frac{{ }^{51} \mathrm{Cr}}{}$ \\
\hline 1 & 85.2 & 80.3 & 86.6 & 98.6 & 109 \\
\hline 2 & 94.4 & 81.0 & 84.9 & 95.8 & 92.4 \\
\hline 3 & 94.9 & 88.5 & 87.6 & 101 & 104 \\
\hline Average & 91.5 & 83.3 & 86.4 & 98.5 & 101.8 \\
\hline
\end{tabular}

BEHAVIOR AND TRANSPORT OF RADIONUCLIDES IN THE COLUMBIA RIVER BETWEEN HANFORD AND VANCOUVER, WASHINGTON* ${ }^{*}$. W. Perkins, J. L. Nelson, and W. L. Haushild**

A study of the behavior and transport by the Columbia River of the 11 radionuclides ${ }^{46} \mathrm{Sc},{ }^{51} \mathrm{Cr},{ }^{54} \mathrm{Mn},{ }^{58} \mathrm{Co},{ }^{59} \mathrm{Fe},{ }^{60} \mathrm{Co},{ }^{65} \mathrm{Zn},{ }^{95} \mathrm{Zr}-{ }^{95} \mathrm{Nb}$, ${ }^{106} \mathrm{RU},{ }^{124} \mathrm{Sb}$ and ${ }^{140} \mathrm{Ba}$ was performed in the river reach between Pasco, Washington and Vancouver, Washington, during the period January, 2965. This extensive study of radionuclide behavior was accomplished by direct counting of water salts and filter samples on a multidimensional gamma-ray spectrometer and represents the first measurements of the interactions of most of these radionuclides $i n$ the river. Through this multiple tracer technique the specific and relative behaviors of the 11 radionuclides in regard to their sorption by and movement with suspended particulates in the Columbia River were determined. Also, depletion of these radionuclides from the river during transport and the radionuclide inventory in the stream bed for the river reach between Pasco, Washington and Vancouver, Washington were estimated.

* to be published in Limnology and oceanography.

**U. S. Geological Survey, Portzand, Oregon

NATURAL AND ARTIFICIAL RADIONUCLIDE CONCENTRATION IN FARM PRODUCE IRRIGATED WITH COLUMBIA RIVER WATER-R. W. Perkins

The Hanford reactors introduce smalz amounts of several radionuclides into the Columbia River and some of these can be detected in farm produce irrigated with this water. The concentration of the 14 natural and artificial radionuclides including $22 \mathrm{Na}, 40 \mathrm{~K}, 46 \mathrm{Sc}, 51 \mathrm{Gr}, 54 \mathrm{Mn}, 60 \mathrm{Co}$,
$65 \mathrm{Zn}, 95 \mathrm{Zr}-95 \mathrm{Nb}, 106 \mathrm{Ru}, 134 \mathrm{Cs}, 13 \mathrm{Cs}, 144 \mathrm{Ce}, 226 \mathrm{Ra}$, and $228 \mathrm{Th}$ have been measured in sprinkler-irrigated and ditch-irrigated farm produce and compared with the concentrations of these radionuclides in iragigation water, soiz, and fallout. The artificial radionuclides ${ }^{46}{ }_{S C}{ }^{5}{ }_{C r}$, $60^{\circ}$ and $65 \mathrm{Zn}$ are mainly from irrigation water and are much higher in the sprinkler-irrigated than in the ditch-irrigated produce. The other artificial radionuclides are from both fallout and irrigation water. Concentrations of the natural radionuclides greatzy exceed those of artificial radionuclides in the produce.

Radionuclides enter plants and thus man's food supply from many sources.
Natural radionuclides present in the soil, including uranium, thorium, and 
their daughters and ${ }^{40} \mathrm{~K}$ are taken up directly from the soil. On decay of the natural ${ }^{222}$ Rn in the air, its longlived daughters ${ }^{210} \mathrm{~Pb}(21 \mathrm{yr})$ and ${ }^{210}$ Po $(138 \mathrm{~d})$ are deposited on the soil and on the leaves of vegetation and taken into the plant. Other airborne radionuclides which have resulted from nuclear weapons testing or which are produced continuously in the atmosphere by cosmic ray spallation reactions are similarly deposited and taken into plants.

At Hanford the nuclear reactors are cooled with Columbia River water and on subsequent discharge of this water to the river small amounts of several nuclides with induced radioactivity enter the river. The use of the Columbia River for irrigation at downstream locations provides another route for radionuclide entry into farm produce.

The observed concentrations of ${ }^{65} \mathrm{Zn}$ and ${ }^{51} \mathrm{Cr}$ in farm produce which was irrigated with Columbia River water were reported several years earlier. $(108,109)$ However, at the time of those earlier studies, sufficiently sensitive techniques were not available to allow a detailed study of the uptake of natural and other artificial radionuclides. The availability of a multidimensional gamma ray spectrometer has now allowed the direct instrumental measurement of 14 radionuclides in farm produce.

To place the radionuclide content of farm produce in proper perspective, the concentrations of both natural and artificial radionuclides in several types of produce and fodder has been measured. The study has included the measureme 40 of the three natural radionuclides $\mathrm{K},{ }^{226} \mathrm{Ra}$, and ${ }^{228} \mathrm{Th}$, and the
11 artificial radionuclides ${ }^{22} \mathrm{Na} .{ }^{46} \mathrm{Sc}$. ${ }^{51} \mathrm{Cr},{ }^{54} \mathrm{Mn},{ }^{60} \mathrm{Co},{ }^{65} \mathrm{Zn},{ }^{95} \mathrm{Zr}-{ }^{95} \mathrm{Nb}$, ${ }^{106} \mathrm{Ru}, 134 \mathrm{Cs}, 137 \mathrm{Cs}$, and ${ }^{144} \mathrm{Ce}$. The origin of the ${ }^{\angle L} \mathrm{Na}$ is mainly nuclear weapons testing, with a small fraction resulting from cosmic ray spallation of argon in the atmosphere. (110) ${ }^{46} \mathrm{Sc} .{ }^{51} \mathrm{Cr} .{ }^{60} \mathrm{Co}$. and ${ }^{65} \mathrm{Zn}$ are main $1 \mathrm{v}$ from irrigation water ${ }^{(101)}$ (introduced by the Hanford reactors) while ${ }^{54} \mathrm{Mn}$, ${ }^{95} \mathrm{Zr}-{ }^{95} \mathrm{Nb},{ }^{106} \mathrm{Ru},{ }^{134} \mathrm{Cs}$, and ${ }^{144} \mathrm{Ce}$ are from both the Hanford reactors and fallout.

Since many of the radionuclides of interest were present at only a few disintegrations per minute per kilogram of produce, samples of a kilogram or more were used wherever possible. Sample preparation involved a slow, low temperature ashing of the sample (400 to $500{ }^{\circ} \mathrm{C}$ ) followed by pressing of the resulting ash into a standard geometry pellet ( $1 / 2$ in. thick by 2 in. diam) for counting. All samples were counted for $1000 \mathrm{~min}$ on a multidimensional gammaray spectrometer. (103)

The observed concentrations of the 14 radionuclides in produce, irrigation water and soil are presented in Table XVI. The measurements include produce irrigated by both ditch and sprinkler systems. The water for the ditch irrigation was pumped directly from the river while that for the sprinkler ir rigation passed through the Richland City water treatment plant, with a resulting decrease in its radionuclide content, prior to its use. Several of the radionuclide measurements were made a few months after sample collection and this limited the number of samples on which ${ }^{51} \mathrm{Cr}(26 \mathrm{~d})$ and ${ }^{95} \mathrm{Zr}$ $(65 \mathrm{~d})$ could be measured. Sample composition and radionuclide concentration 
TABLE XVI. Radionuclide Concentration in Farm Produce Irrigated with Columbia River Water

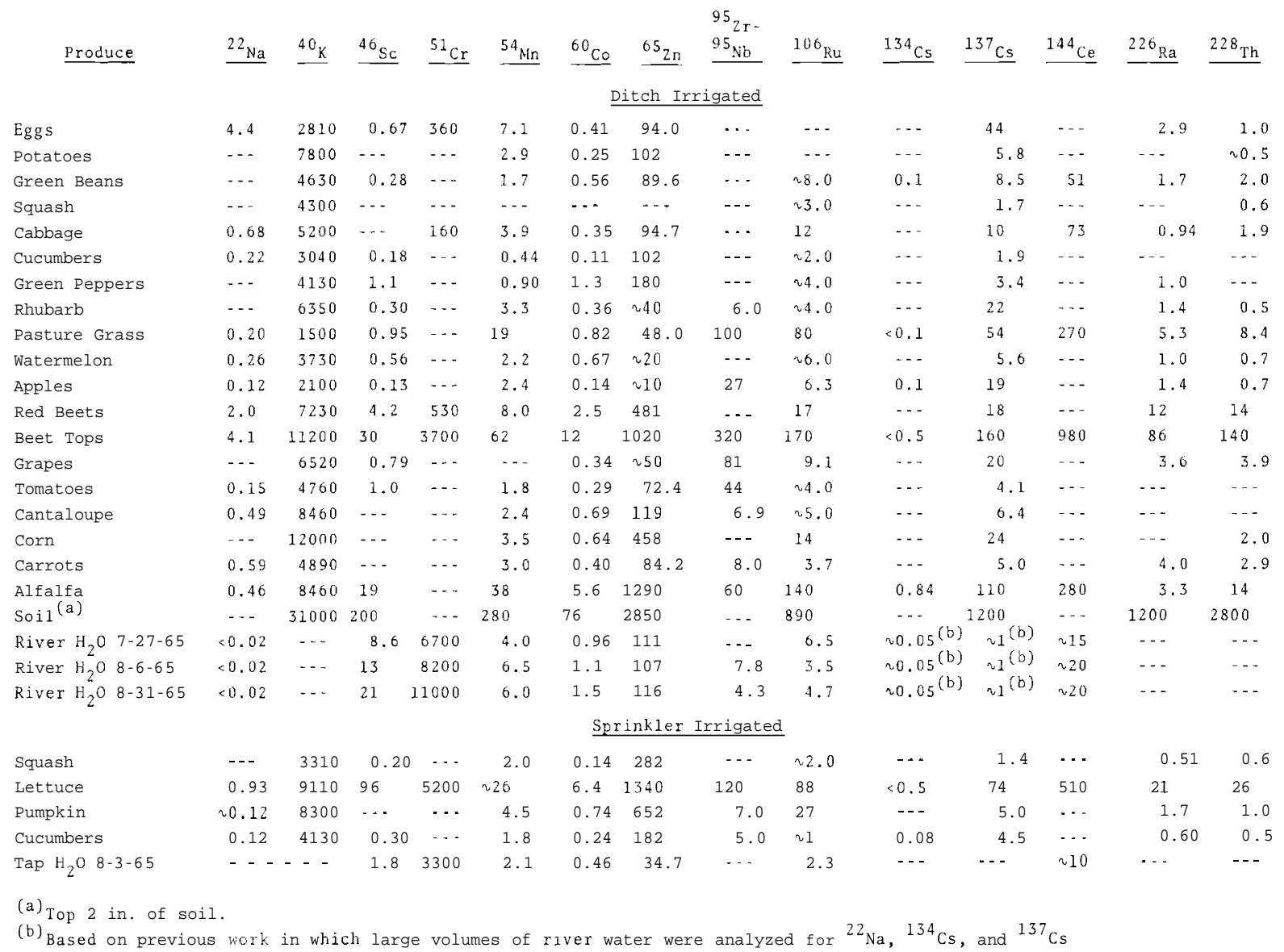

limited the other radionuclides which could be measured in the various samples.

It is apparent that the natural radioactivity due to ${ }^{40} \mathrm{~K}$ is much higher than than due to artificial radionuclides from all sources. The activity density of ${ }^{40} \mathrm{~K}$ in natural potassium is about $1840 \mathrm{dis} / \mathrm{min} / \mathrm{g}$ and the ${ }^{40} \mathrm{~K}$ values thus serve as a measure of the potassium content of the various types of vegetation. The artificial radionuclides present can be roughly divided according to how greatly they are concentrated by the vegetation. This reflects both the affinity of a plant for a radionuclide and its availability for uptake by the plant. In general all of the radionuclides which were present in irrigation water were in much higher concentration in sprinkler-irrigated produce than in the ditch-irrigated produce. This suggests that foliate absorption may play an important part in the uptake of these radionuclides. The beet tops from the ditch-irrigated farm had higher counts than most other products; however, they grow in a manner such that they contact the water during irrigation and foliate absorption may also be important here. Of the radionuclides ${ }^{46} \mathrm{Sc},{ }^{51} \mathrm{Cr},{ }^{60} \mathrm{Co}$, and ${ }^{65} \mathrm{Zn}$, 
which result mainly from irrigation water, the first three have a lower specific activity in most ditch irrigated produce than in the irrigation water. The beets and the beet tops are exceptions, as mentioned previously. The 65 zn concentrations indicate some preferential uptake particularly by corn. Previous studies have shown that ${ }^{65} \mathrm{Zn}$ is preferentially concentrated by corn and wheat. (109) The ${ }^{46} \mathrm{Sc},{ }^{51} \mathrm{Cr},{ }^{60} \mathrm{Co}$ and ${ }^{65} \mathrm{Zn}$ were higher in the sprinkler-irrigated crops; however, only a limited group of produce was available for comparison. The specific activity of the ${ }^{65} \mathrm{Zn}$ in these crops was about 5 to 40 times that in the irrigation water. The specific activity of the ${ }^{46} \mathrm{Sc}$ and ${ }^{60} \mathrm{Co}$ were about 50 to 15 fold higher respectively, on lettuce than in irrigation water. The specific activity of ${ }^{51} \mathrm{Cr}$ was comparable on the lettuce and in the irrigation water, and the radioisotope was not detected on the other produce.

The ${ }^{22} \mathrm{Na}$ becomes available to plants from fallout and is not present in a significant amount in irrigation water. Previous studies(110) had shown relatively high concentrations of ${ }^{22} \mathrm{Na}$ in meat, milk, and wheat and smaller amounts in vegetables. The results from this present study are in agree ment with prior observations and indicate that eggs are another important source of ${ }^{22} \mathrm{Na}$ in the diet. The radionuclides ${ }^{54} \mathrm{Mn},{ }^{95} \mathrm{Zr}-{ }^{95} \mathrm{Nb},{ }^{106} \mathrm{Ru},{ }^{134} \mathrm{Cs}$, and ${ }^{144} \mathrm{Ce}$ are from both fallout and irrigation water. Since there is not a large difference in the activities present on the sprinkler- and ditchirrigated produce it appears that ir- rigation water is not a major source of the radionuclides. For ${ }^{134} \mathrm{Cs}$ and ${ }^{137} \mathrm{Cs}$, their ratio provides a clue as to their origin. The ratio of ${ }^{134} \mathrm{Cs}$ to ${ }^{137} \mathrm{Cs}$ in the Columbia River is about $1: 20^{\text {(111) }}$ below the Hanford reactors, while that in fallout is about 1:500. Since most of the measurements that could be made showed at least 100 -fold more ${ }^{137}$ Cs than ${ }^{134}$ Cs this would indicate that the radiocesium is mainly from fallout.

The radionuclides ${ }^{226} \mathrm{Ra}$ and ${ }^{228} \mathrm{Th}$ in the produce are members of the ${ }^{238} \mathrm{U}$ and ${ }^{228}$ Th chains, respectively, and come from the natural radioactivity in the soil. While the ${ }^{226} \mathrm{Ra}$ must be taken into the plant as radium, the ${ }^{228}$ Th may have resulted from the direct uptake of ${ }^{228} \mathrm{Ra}(6.7 \mathrm{yr})$ and its subsequent decay in the plants to form ${ }^{228} \mathrm{Th}$. The concentrations of these radionuclides are low in produce because of their relatively low concentration in soil. Very high natural radioactivity levels have been observed in plants from regions of high natural radioactivity in the soil.

Although the concentration of all of the radionuclides observed in this study are extremely low compared with Maximum Permissible Concentration values (112) for drinking water, they are important in understanding radionuclide behavior in the environment. These values plus the known retentions and biological half-lives of the radionuclides allow one to estimate body burdens which would result from use of these foods in the diet. In addition, the relationships between the radionuclide concentrations in irrigation water, soil, and air, and that resulting in farm produce, allow one to 
estimate what radionuclide levels in the environment would produce undesir- ably high radionuclide concentrations in farm produce.

DISTRIBUTION AND EXCRETION OF TECHNETIUM IN HUMANS*-T. M. Beasley, H. E. Palmer and W. B. Nelp**

Widespread use of ${ }^{99 m}$ Tc as a tracer in medicine and large scale separation of $99 \mathrm{TC}$ from fission product waste require that adequate knowledge of the metabolic properties of technetium be known so that realistic exposure limits can be imposed for the isotopes of interest. This work discusses a metabolic experiment using $95 \mathrm{~m}_{\mathrm{TC}}(60 \mathrm{~d})$ and $96 \mathrm{Tc}(4.3 \mathrm{dl}$ in which long term excretion, retention, and localization of these isotopes were measured using whole body counting techniques. Following intravenous and oral administration, both urinary and fecal excretion rates were measured, and mathematical expressions were derived for technetium elimination. The latter should be helpful in estimating body burdens of the weak-beta emitting ${ }^{99}$ TC following advertent exposure.

\footnotetext{
"Presented at the Annual Health Physics Society Meeting and accepted for publication in $\underline{\text { Health }} \underline{\text { Physics }}$

**irector, Division of Nuclear Medicine, University of Washington Hospital, Seattle, Washington
}

${ }^{210} \mathrm{~Pb}$ AND ${ }^{210} \mathrm{PO}$ IN ALASKAN BIOLOGICAL SAMPLES-T. M. Beas ley and H. E. PaImer

The past two decades have seen an increased effort on the part of research workers in the radiological sciences to identify and measure the natural radiation levels to which man i s subject. These investigations have been prompted in large measure by the advent of radioactive material introduced into the environment from nuclear weapons testing and by the high natural radiation levels that have been discovered in various parts of the world. (113)

More recently, the concentrating of fallout radioisotopes in some ecosystems such as that observed in the Arctic regions for ${ }^{137} \mathrm{Cs}(114)$ and ${ }^{55} \mathrm{Fe}^{(115)}$ have prompted research in the area of "natural fallout," notably the concentrations in the environment of the naturally occurring radioisotopes ${ }^{210} \mathrm{~Pb}(22 \mathrm{yr})$ and ${ }^{210} \mathrm{Po}(138 \mathrm{~d})$.

The present discussion deals with the levels of these latter two isotopes which have been measured in certain biological samples from Alaska.

The most extensive investigation of the natural ${ }^{210} \mathrm{~Pb}$ concentrations in man is that of Holtzman. (116-119) Included in these measurements were some biological materials from Arctic regions(120) which showed relatively high concentrations of this isotope in lichens, caribou bone. and antler. The source of this ${ }^{210} \mathrm{~Pb}$ is undoubtedly the decay of $222 \mathrm{Rn}$ in the atmosphere and the high levels of ${ }^{210} \mathrm{~Pb}$ in the caribou result from a concentration process of the ${ }^{210} \mathrm{~Pb}$ by lichens similar to that observed for ${ }^{137}$ Cs. We have extended 
these measurements to include a variety of biological samples from the Alaskan region and have determined not only the ${ }^{210} \mathrm{~Pb}$ content of these materials but also the ${ }^{210}$ Po concentrations.

${ }^{210}$ Po was determined by first wet ashing the samples in concentrated nitric and perchloric acids and $30 \%$ hydrogen peroxide,, followed by chemical electrodeposition of the polonium from a dilute hydrochloric acid solution after the method of Black. (88) ${ }^{208}$ Po was used for the radiochemical yield measurement through the dissolution and plating procedure, the polonium finally being determined by alpha energy analysis using a silicon diode detector and a 400 channel pulse height analyzer. Since lead is not lost in this procedure, ${ }^{210} \mathrm{~Pb}$ was determined in the residue from the polonium plating by the procedure of $\mathrm{S}$ ill and Willis. (121) $212 \mathrm{~Pb}$ was used for the radiochemical yield measurement and the ${ }^{210} \mathrm{~Pb}$ activity determined by low background beta counting through aluminum absorbers following decay of the ${ }^{212} \mathrm{~Pb}$ and the at tainment of partial equilibrium between $210 \mathrm{~Pb}$ and its daughter, $210 \mathrm{Bi}$.

The identification and origin of the caribou samples which were analyzed and the levels of ${ }^{210} \mathrm{~Pb}$ and 210 Po observed are contained in Table XVII. The values listed for the caribou are the concentrations existing at time of slaughter, and show the relative levels of the two isotopes in the various organs of the living animal. Since isotopic tracers were used as yield determinants, the probable errors in the determinations

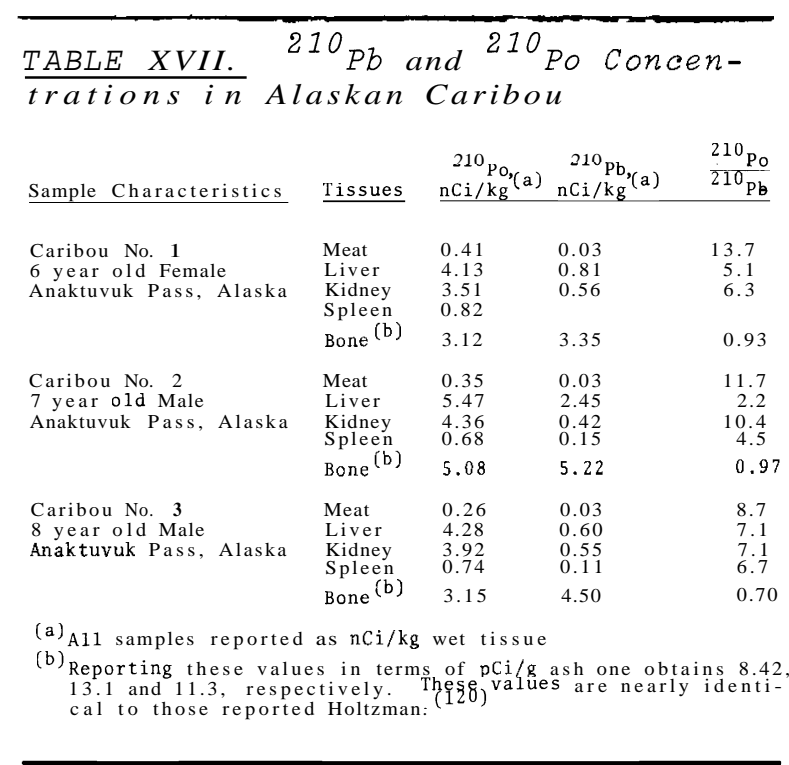

are due almost exclusively to counting statistics. Care was taken to insure that probable errors from this source were less than $10 \%$ for the measurements of both isotopes at the $90 \%$ confidence level.

Table XVIII contains the results of the ${ }^{210}$ Po determination in composite lichen sample and certain foodstuffs used by Eskimos living on the western coastof Alaska. ${ }^{210} \mathrm{~Pb}$ results are not

TABLE XVIII. 210 Po Concentrations in Other Selected Aslaskan Samples

$\begin{array}{lc}\text { Sample } & \begin{array}{c}{ }^{210} \mathrm{Po}, \\ \mathrm{nCi} / \mathrm{kg}(\mathrm{a})\end{array} \\ { } } & 4.70 \\ \text { Salmon (c) } & 0.026 \\ \text { Butterfish } & 0.007 \\ \text { Whitefish } & 0.007 \\ \text { Seal Meat } & 0.220 \\ \text { Seal Liver } & 0.880 \\ \text { Polar Bear Meat } & 0.008\end{array}$

\footnotetext{
(a) Wet weight

(b) The lichen sample listed in a composite sample taken from Anaktuvuk Pass, Alaska. No distinction can be made as to the type of lichen present. Furthermore, the concentration reported here corresponds to a value of 14.9 nCi $/ \mathrm{kg}$ dry lichen, which is in general agreement with that reported by Holtzman. (120)

(c) Average of four salmon.
} 
included for these particular samples since they are used here only as an indication of the relative levels of ${ }^{210}$ Po to which these particular individuals might be exposed. However, in those samples such as seal meat and seal liver, one might expect the activity to be supported in part by ${ }^{210} \mathrm{~Pb}$.

Several common foodstuffs (meats, grain products,eggs, etc.) from local foodmarkets have been analyzed in this laboratory; (122) the results show that generally the levels of ${ }^{210}$ Po in these foods are some $1 / 10$ to $1 / 100$ of those in caribou flesh, seal meat, and salmon listed in Tables XVII and XVIII.

Although more samples must be analyzed to obtain a clearer picture of the ${ }^{210} \mathrm{~Pb}-{ }^{210} \mathrm{Po}$ concentrations in the

Alaskan ecosystem, certain conclusions can be drawn from the data of Tables XVII and XVIII. First, it is clear that the caribou have been subjected to higher than the usual, world-average levels of internal radiation. If the concentrations of the ${ }^{210}$ Po in the various organs of the caribou are constant at the levels listed in Table XVII, the dose rate to these various organs would be those shown in Table XIX. Since this dose is due to alpha

TABLEXIX. Approximate Dose Rates to Caribou Organs from 210 Po

\begin{tabular}{lcc} 
& $\begin{array}{c}\text { Average }{ }^{210} \text { Po Content, } \\
\text { Tissue }\end{array}$ & $\begin{array}{c}\text { Dose } \\
\text { rad } / \mathrm{y} \text { Y }\end{array}$ \\
\cline { 2 - 2 } Liver & 4.62 & 0.45 \\
Kidney & 4.05 & 0.39 \\
Spleen & 0.74 & 0.07 \\
Bone & 3.78 & 0.37 \\
& & \\
(a) Concentrations are in kilograms wet weight.
\end{tabular}

particles it is probably more effective than an equal dose of X-rays due to a higher R.B.E. and to a lack of a protraction effect.

Since certain of the Alaskan natives depend upon caribou meat for a large portion of their diet, they will also be subjected to a much higher radiation dose from these isotopes than other United States residents. The problem can be put in better perspective by comparing the ${ }^{210} \mathrm{~Pb}-{ }^{210} \mathrm{Po}$ problem to that of ${ }^{137} \mathrm{Cs}$ arising from nuclear weapons fallout. In making this comparison, we have assumed that the maximum permissible concentrations for ${ }^{137} \mathrm{Cs},{ }^{210} \mathrm{Po}$, and ${ }^{210} \mathrm{~Pb}$ are the same in meat as they are in water. These values are listed in the I.C.R.P. Committee II report(112) as $2.4 \times 10^{-4}$ $\mu \mathrm{Ci} / \mathrm{cm}^{3}, 7 \times 10^{-6}{ }_{\mu \mathrm{Ci} / \mathrm{cm}^{3}}$, and $1 \times 10^{-6}$ $\mu \mathrm{Ci} / \mathrm{cm}^{5}$, respectively. The ratio MPC ${ }^{210} \mathrm{Po} / \mathrm{MPC}{ }^{137} \mathrm{Cs}=30$. Thus, the ${ }^{210}$ Po if present in the same concentration as ${ }^{137}$ Cs would provide 30 times the radiation dose. Similarly, the ratio $\mathrm{MPC}{ }^{210} \mathrm{~Pb} / \mathrm{MPC}{ }^{137} \mathrm{Cs}=200$. Since the average ${ }^{137} \mathrm{Cs}$ content of caribou flesh has recently been measured as approximately $20 \mathrm{nCi} / \mathrm{kg}^{(123)}$ and the ${ }^{210} \mathrm{Po}$ and ${ }^{210} \mathrm{~Pb}$ concentrations tentatively determined to be 0.3 and 0.03 $\mathrm{nCi} / \mathrm{kg}$ respectively, the relative hazard to the Eskimo can be computed for ${ }^{210}$ Po as $(0.3 / 20) 30=0.45$ and for ${ }^{210} \mathrm{~Pb}$ as $(0.03 / 20) 200=0.3$.

Thus, if an Eskimo should consume enough caribou flesh containing the concentrations of ${ }^{137} \mathrm{Cs},{ }^{210} \mathrm{Po}$, and ${ }^{210} \mathrm{~Pb}$ listed above and should reach a maximum permissible body burden of ${ }^{137} \mathrm{Cs}$ as a result, he would also be technically overexposed by some $45 \%$ 
due to ${ }^{210}$ Po and by some $30 \%$ due to ${ }^{210} \mathrm{~Pb}$. The maximum permissible body burden, sustained, which has been recommended by the I.C.R.P. for general populations not exposed to ${ }^{137} \mathrm{Cs}$ in the course of their work is $3 \mu \mathrm{Ci}$. Some Alaskan natives already have. ${ }^{137} \mathrm{Cs}$ body burdens approaching $3 \mu \mathrm{Ci}$. These high body burdens are known to vary according to the seasonal changes that occur in the ${ }^{137}$ Cs content of the caribou. It is possible however, that even with the seasonal variations that occur in the ${ }^{137} \mathrm{Cs}$ body burdens, the permissible exposure of these individuals might be exceeded for periods of time due to the presence of ${ }^{210} \mathrm{~Pb}$ and 210 Po in their diet.

There is direct evidence that indeed Eskimos contain much higher concentrations of ${ }^{210} \mathrm{~Pb}$ and ${ }^{210}$ Po than do other United States residents. Hi11(91) has measured the ${ }^{210} \mathrm{~Pb}$ content of a rib bone obtained from a Canadian Eskimo and has observed a concentration of $2.3 \mathrm{pCi} / \mathrm{g}$ ash, which is some 15 times greater than the average ${ }^{210} \mathrm{~Pb}$ bone content observed by Holtzman (116) in surgery and autopsy specimens obtained from individuals residing in Illinois. Secondly, measurements of ${ }^{210}$ Po in urine specimens obtained from natives living at Anaktuvuk Pass, Alaska, showed average concentrations of $7.0 \mathrm{dis} / \mathrm{min}$ per 1.4 liters, a concentration of some 230 times that observed by Hursh and Sultzer ${ }^{(125)}$ for normal individuals who were exposed only to normal environmental levels of ${ }^{210} \mathrm{~Pb}$ and ${ }^{210} \mathrm{Po}$. If we use the animal data of Fink (126) which implies that $0.1 \%$ of the ${ }^{210}$ Po body content is excreted per day in the urine and assuming the natives are at equilibrium with their intake, the calculated average ${ }^{210}$ Po body burden of the people measured would be $3500 \mathrm{pCi}$, or approximately $10 \%$ of a maximum permissible body burden of ${ }^{210}$ Po under the definition given above for ${ }^{137} \mathrm{Cs}$.

More detailed measurements of the ${ }^{210} \mathrm{~Pb}$ and ${ }^{210} \mathrm{Po}$ concentrations of the Eskimo diet and of the urinary excretion of these two isotopes in Alaskan natives are presently underway which will further define levels to which these particular individuals are exposed.

THE DISTRIBUTION OF NATURAL AND ARTIFICIAL RADIONUCLIDES THROUGH THE FOOD CHAIN IN ALASKA-R. W. Perkins and W. C. Hanson

The uptake of fallout radionuclides by Alaskan lichens results in a concentration of ${ }^{137}$ Cs 100 times higher in some Alaskan natives than in residents of the mainland states. $(114,124)$ The lichens which concentrate fallout directly from the air, or from rain or melted snow, serve as a main source of food for caribou and the caribou are a principal item in the diet of the Alaskan Eskimo. Lichens serve not only to concentrate ${ }^{137} \mathrm{Cs}$ but all of the airborne radionuclides. This relatively high concentration of radionuclides at this first step in the food chain provides an excellent opportunity to observe the behavior of radionuclides in their movement through the food chain to man. Since caribou are the main item in the diet for the 
Alaskan wolf as well as the Eskimo, measurements of radionuclides in wolf tissue were used to estimate those which would be present in the Eskimo. A 11 of the measurements considered in this study including the measurement of 15 radionuclides in the lichens were made by direct counting on a multidimensional gamma ray spectrometer. (103)

In Table XX the concentrations of 10 fallout radionuclides measured in a ir at Point Barrow, Alaska, are compared with those observed in lichen samples collected near Anaktuvuk Pass. These data show that the relative concentrations of the airborne radionuclides are similar to those on the lichens and that relatively little, if any discrimination is exercised in their uptake by the lichens. It is apparent, however, that the short-lived radionuclides ${ }^{95} \mathrm{Zr}-^{95_{\mathrm{Nb}}}$ and ${ }^{88} \mathrm{Y}$ are present on the lichens in relatively low concentrations compared to their abundance in air while the long-lived radionuclides ${ }^{60} \mathrm{Co}$ and ${ }^{137} \mathrm{Cs}$ are present in relatively high concentrations on the lichens. This would be expected since short-lived radionuclides on the lichens would reflect current and recent air concentrations while long-lived radionuclides would average the air concentrations over several years and include periods of high radionuclide concentrations. At the time these air and lichen samples were collected, $1 \mathrm{~kg}$ of lichen contained about as much of the fallout radionuclides as $10^{7} \mathrm{ft}^{3}$ of air near ground level. The relatively high concentrations of ${ }^{134} \mathrm{Cs}$ in the lichen sample are in accord with previous observations; they showed that the ${ }^{134} \mathrm{Cs}$ to ${ }^{137}$ Cs ratio in fallout from the 1961-1962 nuclear test series was much lower than the ratio previously present in the Alaskan ecosystem. (110)

Table XXI summarizes the results of a study in which the concentrations of 15 radionuclides were measured in the three trophic levels: lichen, caribou, and wolf (also bioassay samples

TABLE XX. Comparison of Airborne and Lichen Concentrations of Ten Falzout Radionuclides

\begin{tabular}{|c|c|c|c|}
\hline Radionuclide & Half-1 & Life & dis/min per $10^{7} \mathrm{ft}^{3}(\mathrm{a}$ \\
\hline${ }^{22} \mathrm{Na}$ & 2.58 & $y r$ & 22 \\
\hline${ }^{54} \mathrm{Mn}$ & 310 & $d$ & 1890 \\
\hline${ }^{60} \mathrm{Co}$ & 5.27 & $\mathrm{yr}^{2}$ & 21.2 \\
\hline${ }^{88} Y$ & 108 & d & 14.3 \\
\hline${ }^{95} \mathrm{Zr} \cdot{ }^{95} \mathrm{Nb}$ & $65 \&$ & $35 \mathrm{~d}$ & 1920 \\
\hline${ }^{106} \mathrm{Ru}$ & 365 & d & 8850 \\
\hline${ }^{125} \mathrm{Sb}$ & 2.7 & $y r$ & 3470 \\
\hline${ }^{134} \mathrm{Cs}$ & 2.1 & $\mathrm{yr}$ & 5.0 \\
\hline${ }^{137} \mathrm{Cs}$ & 30 & $\mathrm{yr}$ & 4220 \\
\hline${ }^{144} \mathrm{Ce}$ & 285 & d & 17,700 \\
\hline
\end{tabular}

\begin{tabular}{cc}
$\begin{array}{c}\text { Lichen Concentration } \\
\text { dis/min per kilogram }\end{array}$ & dis/min per kilogram Lichen \\
\cline { 1 - 2 } 21 & 0.95 \\
4180 & 2.21 \\
83 & 3.92 \\
10 & 0.70 \\
975 & 0.51 \\
12,400 & 1.40 \\
3830 & 1.10 \\
50 & 10 \\
13,700 & 3.25 \\
35,300 & 1.99
\end{tabular}

(a) Air sample collected during December, 1964.

(b) Lichen sample collected during october, 1964 . 
TABLEXXI. Radionuclides in Lichens, Caribou, Wolf, and Bioassay Samples from Alaska (Units of dis/min per kilogram of wet weight)

\begin{tabular}{|c|c|c|c|c|c|c|c|c|c|c|c|c|c|c|c|c|c|}
\hline Sample Type & No. ${ }^{\text {(d) }}$ & $\begin{array}{c}\begin{array}{c}\text { Collection } \\
\text { Date }\end{array} \\
\end{array}$ & ${ }^{22} \mathrm{Na}$ & $\underline{40} \mathrm{~K}$ & ${ }^{54} \mathrm{Mn}$ & ${ }^{60} \mathrm{Co}$ & ${ }^{65} \mathrm{Zn}$ & ${ }^{88} \mathrm{Y}$ & $\begin{array}{l}95 \mathrm{Zr}- \\
95 \mathrm{Nb} \\
\end{array}$ & ${ }^{106} \mathrm{Ru}$ & $110 \mathrm{~m}_{\mathrm{Ag}}$ & ${ }^{125} \mathrm{Sb}$ & ${ }^{134} \mathrm{Cs}$ & ${ }^{137} \mathrm{Cs}$ & ${ }^{144} \mathrm{Ce}$ & ${ }^{226} \mathrm{Ra}$ & $228 \mathrm{Th}$ \\
\hline Lichens (a) & (1) & $10-15-64$ & 21 & 1070 & 4180 & 83 & (e) & 10.0 & 975 & 12400 & 12.0 & 3830 & 50 & 13700 & 35300 & 26 & 15 \\
\hline Lichens $(b)$ & (1) & $1-19-65$ & 33 & 560 & 5130 & 92 & $(e)$ & 7.7 & 560 & 14900 & 7.6 & 4990 & 38 & 18100 & 35000 & 35 & 15 \\
\hline Lichens ${ }^{(C)}$ & (1) & $1-19-65$ & 20 & 500 & 3130 & 83 & (el & 5.2 & 160 & 9800 & 6.4 & 3500 & 28 & 12500 & 26700 & 28 & 14 \\
\hline Caribou flesh & (1) & $10-15-64$ & 56 & 6660 & (el & 1 & 42 & (el & (e) & (e) & (e) & (e) & 30 & 23600 & (e) & (e) & (e) \\
\hline Caribou flesh & (1) & $1-20-65$ & 640 & 6120 & 22 & 2 & 82 & (e) & (e) & (e) & 2 & (e) & 53 & 43600 & (e) & (e) & 0.7 \\
\hline Caribou liver & (3) & $1-19-65$ & 800 & 5290 & 340 & 72 & 84 & (e) & (e) & 620 & 630 & (e) & 31 & 23100 & (e) & (e) & 7.3 \\
\hline Caribou liver & (3) & $2-3-65$ & 580 & 5650 & 500 & 82 & 72 & (e) & (e) & 460 & 490 & (e) & 37 & 26800 & (e) & (e) & 7.3 \\
\hline Caribou kidney & (2) & $1-20-65$ & 1420 & 4340 & 150 & 34 & 65 & (e) & (e) & 760 & 17 & (e) & 61 & 46000 & (el & (e) & 17 \\
\hline Caribou kidney & (2) & $2-12-65$ & 1110 & 4810 & 220 & 35 & 76 & (el & (e) & 680 & 3 & (e) & 66 & 49900 & (e) & (e) & 9.0 \\
\hline Caribou bone & (1) & $10-15-64$ & 300 & 1240 & (e) & (e) & (e) & (e) & (e) & (e) & (e) & (e) & 6 & 1770 & (e) & (e) & 34 \\
\hline Caribou bone & (1) & $1-20-65$ & 2500 & 2090 & 75 & 8 & 100 & (el & (e) & (e) & 21 & (e) & 34 & 13100 & (e) & (e) & 42 \\
\hline Wolf flesh & (1) & $2-8-65$ & 610 & 6250 & 73 & 3 & 150 & $(e)$ & (e) & (e) & 1 & (e) & 96 & 75800 & (e) & (e) & 0.9 \\
\hline Wolf liver & (1) & $2-8-65$ & 720 & 5670 & 250 & 12 & 70 & (e) & (e) & (e) & 5 & (e) & 61 & 48800 & (e) & (e) & 4.2 \\
\hline Wolf kidney & (2) & $1-21-65$ & 1040 & 2940 & 290 & 20 & 120 & (e) & (e) & 640 & 14 & (e) & 9 & 4000 & (e) & (e) & 7.3 \\
\hline Wolf bone & (1) & $2-8-65$ & 2200 & 1200 & 53 & 5 & 200 & (e) & (e) & (e) & 36 & (e) & 26 & 14100 & (e) & (e) & 7.2 \\
\hline Human urine & (5) & $1-20-65$ & 140 & 1400 & (e) & 0.1 & (e) & (e) & (e) & (e) & (e) & (e) & 8.3 & 6550 & (e) & (e) & (e) \\
\hline
\end{tabular}

(a) Cladonia spp. -Cetraria spp. mixed.

(b) Mostly Cetraria cuculata and Cetraria islandica from beneath $1.5 \mathrm{dm}$ frozen snow, steeply sloping terrain

${ }^{(c)}$ Mostly Cladonia alpestris from beneath $7 \mathrm{dm}$ frozen snow, rolling terrain.

from man). $\quad 40_{K},{ }^{226} \mathrm{Ra}$, and ${ }^{228} \mathrm{Th}$ are naturally occurring radionuclides and serve as a baseline for comparison of the concentrations of the artificial radionuclides. $\quad{ }^{22} \mathrm{Na}$ is a naturally occurring radionuclide produced by cosmic ray spallation of atmospheric argon; however, large amounts of this radionuclide were produced during the 1961-1962 nuclear test series and increased the atmospheric ${ }^{22} \mathrm{Na}$ levels by more than an order of magnitude. (127) of the other artifical radionuclides. ${ }^{95} \mathrm{Zr}-{ }^{95} \mathrm{Nb}, 106 \mathrm{Ru},{ }^{125} \mathrm{Sb},{ }^{134} \mathrm{Cs}$, and ${ }^{144} \mathrm{Ce}$ are fission products while ${ }^{54} \mathrm{Mn}$, ${ }^{60} \mathrm{Co},{ }^{65} \mathrm{Zn},{ }^{88} \mathrm{Y},{ }^{110 \mathrm{~m}} \mathrm{Ag}$, and ${ }^{134} \mathrm{Cs}$ are activation products which were produced during the nuclear testing.

These measurements of the natural accumulation in animal tissue are the first for some of the radionuclides and show the movement through the food chain relative to the well established behavior of ${ }^{137} \mathrm{Cs}$. The data show a distinct difference in the behavior of ${ }^{22} \mathrm{Na}$ relative to ${ }^{137} \mathrm{Cs}$. While the ${ }^{137} \mathrm{Cs}$ in the caribou and wolf organs have specific activities of up to a few times that of the lichens, the ${ }^{22} \mathrm{Na}$ is 10 to 100 times higher in most of the animal organs than in the lichens. This situation would require that the combination of the uptake (through the gastrointestinal tractl plus the biological halflife for ${ }^{2} \angle \mathrm{Na}$ be several times that of 137 Cs. Since this is reportedly not the case, (112) the reason for the high ${ }^{22} \mathrm{Na}$ levels in the animals is not clear but is receiving further study.

The concentration of the radionuclide in the muscle tissue of the caribou and wolf is about 1/100 of that in the lichens; however, ${ }^{54} \mathrm{Mn}$ is 
preferentially concentrated by certain organs of those animals. Its concentration in the liver, kidney, and bone ranges from 3 to 30 times that in the muscle tissue.

The ${ }^{60}$ Co concentration in caribou muscle tissue is only 1 to $3 \%$ of that in the lichen while its concentration in the liver, kidney, and bone are comparable to that in the lichen. The ${ }^{60}$ Co concentrations in the organs of the wolf are mostly lower than in the caribou. The ${ }^{65} \mathrm{Zn}$ concentrations in the caribou and wolf do not show large variations between the organs and are generally higher in the wolf. The ${ }^{65} \mathrm{Zn}$ in the lichen was not measured.

The uptake of ${ }^{106} \mathrm{Ru}$ through the gastrointestinal tract wall is reported to be very low; (112) however, a sufficient amount does enter the blood stream to provide substantial concentrations in the liver and kidney. The ${ }^{106} \mathrm{Ru}$ concentration in the liver is about $5 \%$ of that in the lichens; therefore, its uptake and retention by this organ is about half that of ${ }^{54} \mathrm{Mn}$. The relative concentration of ${ }^{106} \mathrm{Ru}$ in the kidney was approximately the same as that in the liver.

${ }^{110 \mathrm{~m}} \mathrm{Ag}$ has only recently been reported to be concentrated in the liver of marine organisms (127) and the measurements present here are the first observations of its accumulation in the terrestrial ecosystem. Of the animal organs that were measured, its concentration in liver tissue was by far the highest, ranging from about 50 to 100 times that in the lichens. 137 Although numerous measurements of ${ }^{1} \mathrm{Cs}$ have been made throughout the Alaskan ecosystem, only a few measurements of ${ }^{134} \mathrm{Cs}$ and its ratio to ${ }^{137} \mathrm{Cs}$ have been made.

Table XXII summarizes our measurements of these ratios in Alaska and compares them with measurements by Liden and Anderson in Sweden. These data show a continuing decrease in the ${ }^{134} \mathrm{Cs}$ to ${ }^{137}$ Cs ratio over the past 5 years.

In Figure 86 these ratios of ${ }^{134} \mathrm{Cs}$ to ${ }^{137} \mathrm{Cs}$ are plotted and compared with $\frac{\text { TABLE XXII. }}{1961-1965} \quad$ Ratios of ${ }^{134}$ Cs to ${ }^{137}$ Cs in Arctic People and Caribou/Reindeer Flesh,

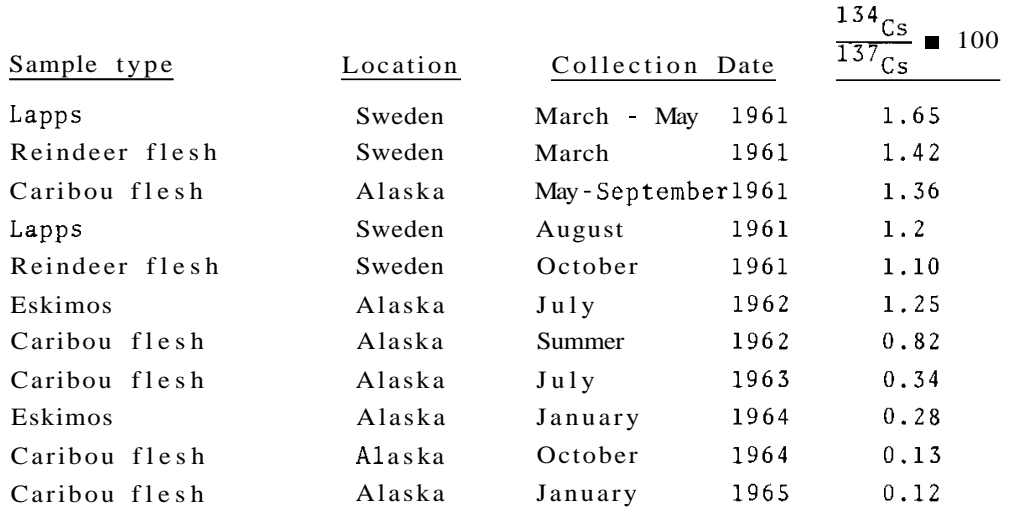

\begin{tabular}{l}
\multicolumn{2}{c}{ Reference } \\
Lidén E Anderson, 1962 \\
Lidén \& Anderson, 1962 \\
Perkins \& Nielsen, 1965 \\
Lidén 4 Anderson, 1962 \\
Lidén \& Anderson, 1962 \\
Palmer \& Perkins, 1963 \\
Palmer 4 Perkins, 1963 \\
Perkins \& Nie1sen, 1965 \\
Perkins 4 Nielsen, 1965 \\
This paper \\
This paper
\end{tabular}




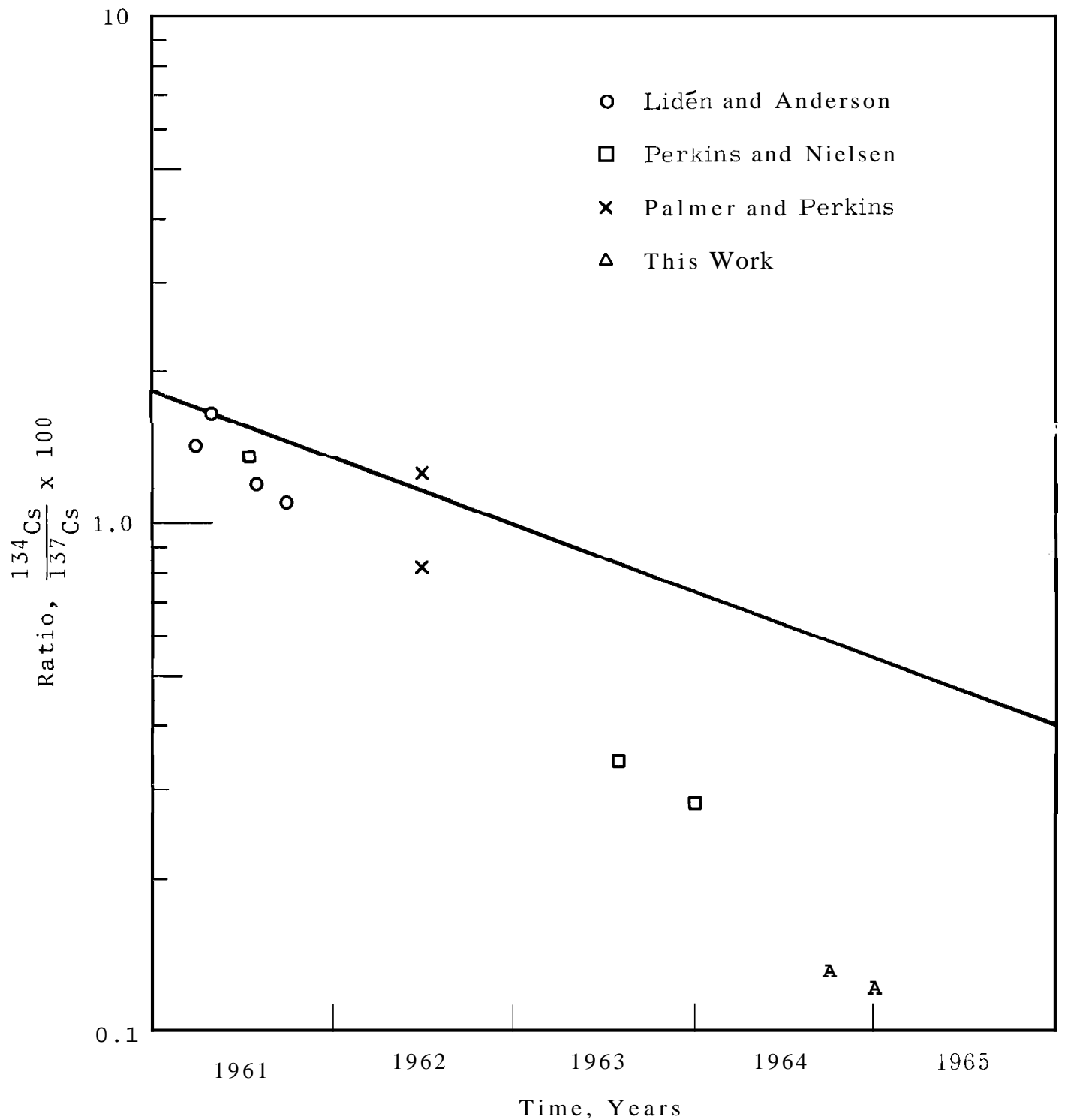

FIGURE 86. Comparison of the Observed ${ }^{134} \mathrm{Cs}$ to ${ }^{137} \mathrm{Cs}$ Ratio with the Calculated Change in Ratio due to Normal Decay of a Mixture

a curve representing the ratios that would be present as a function of time if one started with the observed ratio of 0.0165 for March through May of 1961. The change in the observed ratio with time produces a curve which is much lower than that which results from the decay of the initial $0.0165: 1$ ratio of ${ }^{134} \mathrm{Cs} /{ }^{137} \mathrm{Cs}$. Therefore the radioces ium added during the 1961-1962 test was lower in ${ }^{134} \mathrm{Cs}$ than that present in the ecosystem prior to these tests. 
ULTRA SENSITIVE MEASUREMENT OF RADIONUCLIDES AND TRACE ELEMENT CONSTITUENTS IN THE OCEAN BY MULTIDIMENSIONAL GAMMA-RAY SPECTROMETRY-R. W. Perkins, D. E. Robertson and H. G. Rieck

An understanding of the behavior of the ocean's trace elements and of its natural and artificial radionuclies is essential in obtaining a clear picture of the chemical, biological, and physical processes which occur therein. The extensive use of these natural tracers of oceanic processes has been greatly limited by the extreme difficulties involved in their measurements. Measurements of most of the trace elements and radionuclides in sea water require rather elaborate chemical and/ or instrumental methods, $(129-133)$ and the measurement of a large group of elements or radionuclides in a given sample has been a major analytical problem. The advantages of multiple tracer techniques are obvious, particularly in the oceans where one is interested in comparing specific properties, concentrations, or reaction rates of one element or compound with others. Our developments in multidimensional gamma-ray spectrometry $(98,102,109,127,134)$ have provided a means whereby a dozen or more radionuclides can be directly measured with good precision in many radionuclide mixtures. The application of this new technique to the analysis of ocean water has been very successful. Studies of sea water constituents have shown that direct measurements without chemical separation of numerous radionuclides can be made. Also, following neutron activation of a 0.1 to $0.5 \mathrm{~m}]$ sample of sea water it is possible to make a direct measurement of the ten trace elements strontium, rubidium, iron, zinc, cobalt, antimony, silver, scandium, and uranium from a single count on the multidimensional gamma-ray spectrometer. This technique provides the first opportunity to measure and observe the behavior of such a large group of trace elements simultaneously. It also eliminates the uncertainties associated with many complex chemical separations.

Only a very small sample of sea water $(0.5 \mathrm{~m} 1$ or less $)$ is required for neutron activation and subsequent trace element analysis; however, it is necessary to process a very large volume of sea water to permit measurements of radionuclides which are present at only a few disintegrations per minute per 1000 liters. The present equipment used for this work is shown in Figure 87 and consists of a high volume pump* plus a filtration assembly which contains exchange beds in the top section. The filtration as sembly contains eight 12 in. diam filters and provides a total filtration area of about 800 in. The outlet stream from the filters is circulated through the exchange bed. The tubing is plastic or rubber hose and the filtration system is a 11 polyvinylchloride. The pump, which is of mild steel, is painted inside with a plastic coat to prevent corrosion. For the work considered here, membrane filters with a

*Homolite Model XLS-1-1/2-1, capacity of 4200 gal/hr, Port Chester, N. Y. 


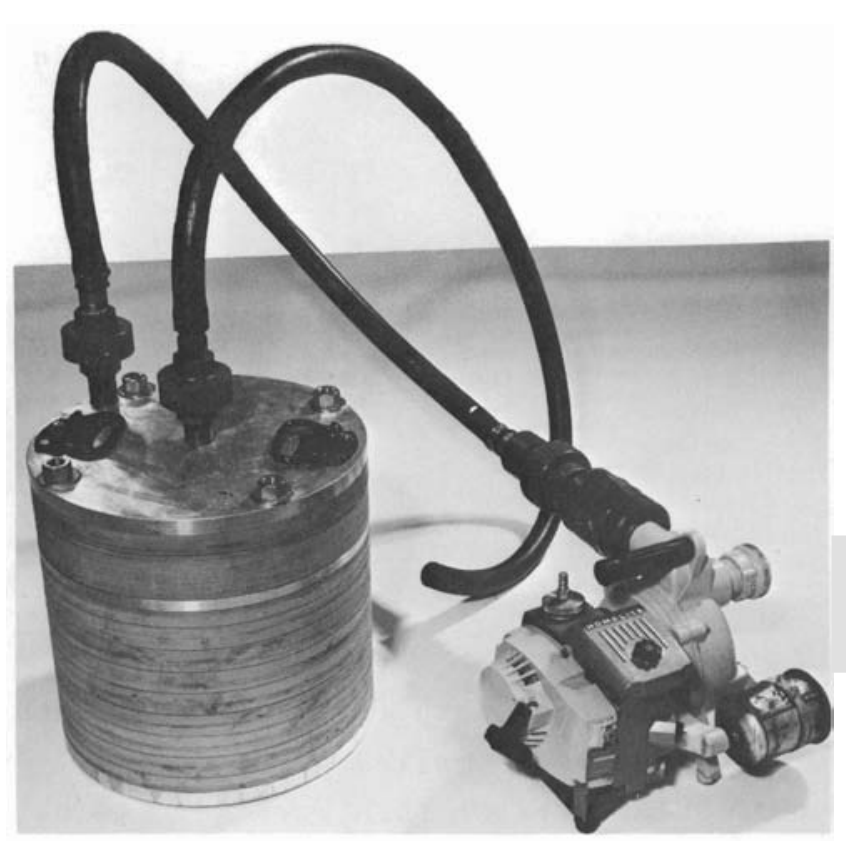

FIGURE 87. Ocean Water Sampling System Showing Assembled PVC Filtration-Sorption Assembly and the High Volume Pump

pore size of $0.3 \mu$ were used with fiber glass backup filters and the flow from two to eight filters was passed through exchange beds which consisted of a 12 in. diam $(500 \mathrm{~g})$ oxidized steel or a $400 \mathrm{~g}$ bed of aluminum oxide (chromatographic grade). In one experiment a $400 \mathrm{~g}$ bed of 50 to 100 mesh KCF-1 potassium hexacyanocobalt(II) ferrate (II) which collects the radiocesium was placed in series with the aluminum oxide bed. Figure 88 shows the KCF-1 being added to the sorption bed section. Figure 89 is a photograph showing the sorption bed and one of the filter frames removed.

Ocean water samples were collected in an all plastic bucket on the end of nylon rope several feet from the ship. The samples were immediately filtered through a membrane filter of $0.45 \mu$ pore size in an all glass

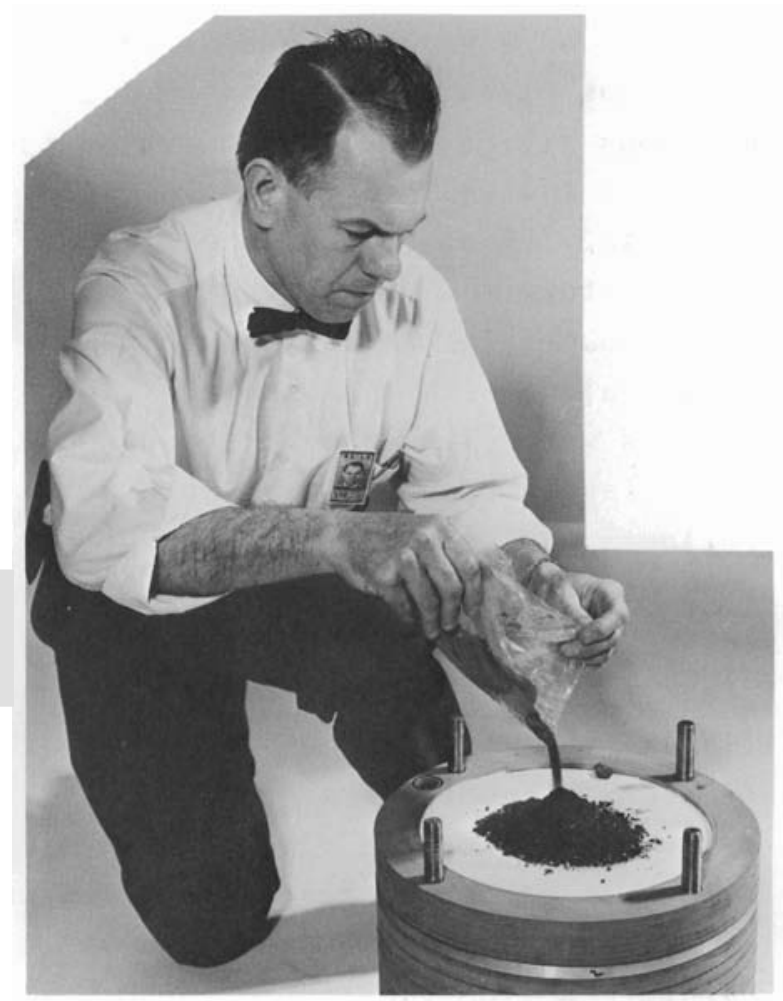

FIGURE 88. Sorption Bed Material Being Added to Top Section of Ocean Sampler

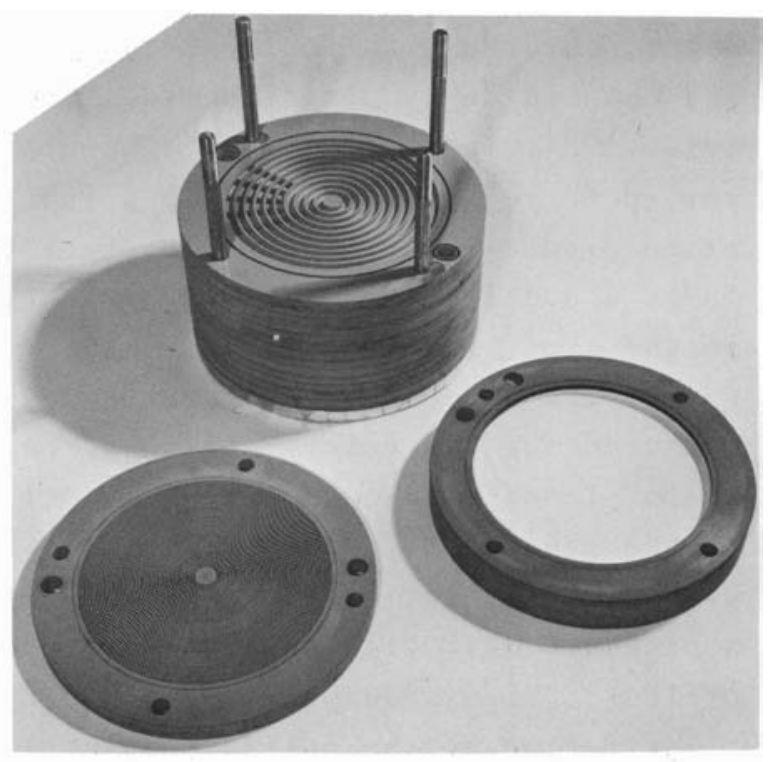

FIGURE 89. Partially Disassembled Ocean Water Samplew; Filter Frame on Left and Sorption Bed on Right 
system, and a $0.100 \mathrm{ml}$ sample of this water was pipetted into a $4 \mathrm{~mm}$ I.D. vitreous silica ampule, evaporated to dryness, and sealed for neutron activation. Standards of each element to be determined were irradiated in a NaC1 matrix of the same size as the sample along with the sample. The ampules were cleaned prior to use by refluxing in doubly distilled $\mathrm{HNO}_{3}$. Following a 1 week irradiation at a flux of greater than $10^{13}$ neutrons/ $\mathrm{am}^{L} \mathrm{sec}$ the samples were allowed to stand for 4 weeks to permit the ${ }^{24} \mathrm{Na}$ $(15 \mathrm{hr})$ and ${ }^{82} \mathrm{Br}(35.7 \mathrm{hr})$ to decay. (These radionuclides are major constituents during the first few weeks and their parent element concentrations, sodium and bromine, can be easily determined from their coincidence gamma-ray spectra as measured on the multidimensional gamma-ray spectrometer). The samples were then quantitatively transferred to the center of a $1 / 4$ in. thick by $11 / 4$ in. diam Lucite sample mount, covered with an identical mount, and counted on a multidimensional analyzer. (98) The Lucite sample mounts served to reduce Bremsstrahlung radiation nroduction which results from the large amounts of ${ }^{35} \mathrm{~S}$ and ${ }^{32} \mathrm{P}$ present in the samples. Table XXII lists the gamma rays and coincidence gamma rays measured for the determination of ten trace elements in ocean water. Most of the radioactive daughters are measured with little interference and with a standard deviation of a few per cent or less. Figure 90 provides a blocked illustration of the multidimensional gamma-ray spectrum showing the energy areas used for the measurement of each radionuclide. The observed concentrations of trace elements in sea water

\begin{tabular}{|c|c|c|c|}
\hline \multicolumn{4}{|c|}{$\begin{array}{l}\text { TABLEXXII. } \\
\text { Other Selected AZaskan Samples }\end{array}$} \\
\hline Element & $\begin{array}{l}\text { Daughter } \\
\text { Radionuclide } \\
\end{array}$ & $\underline{\text { Half-Life }}$ & $\begin{array}{l}\text { Gamma-Ray } \\
\text { Energies, MeV }\end{array}$ \\
\hline Strontium & ${ }^{85} \mathrm{Sr}$ & $65 \mathrm{~d}$ & 0.515 \\
\hline Rubidium & ${ }^{86} \mathrm{Rb}$ & $18.7 \mathrm{~d}$ & 1.077 \\
\hline Iron & ${ }^{59} \mathrm{Fe}$ & $45 \mathrm{~d}$ & 0.191 and 1.097 \\
\hline zinc & 65 zn & $245 \mathrm{~d}$ & 0.511 positron \\
\hline Uranium & ${ }^{140} \mathrm{Ba}-{ }^{140} \mathrm{La}$ & $12.8 \mathrm{~d}$ & $\begin{array}{l}\text { annihilation } \\
0.817 \text { and } 1.597\end{array}$ \\
\hline Cobalt & ${ }^{60} \mathrm{Co}$ & $5.27 \mathrm{y} \mathrm{r}$ & 1.173 and 1.332 \\
\hline Cesium & ${ }^{134} \mathrm{Cs}$ & $2.1 \mathrm{yr}$ & 0.6047 and 0.7964 \\
\hline Antimony & ${ }^{124} \mathrm{Sb}$ & $60 \mathrm{~d}$ & 0.603 and 1.69 \\
\hline Silver & ${ }^{110 m^{A g}}$ & $253 \mathrm{~d}$ & 0.656 and $2.27^{(a)}$ \\
\hline Scandium & ${ }^{46} \mathrm{Sc}$ & $84 \mathrm{~d}$ & 0.887 and 1.119 \\
\hline (a) Sum & & ma & \\
\hline
\end{tabular}

samples have shown fair agreement with the averages compiled by Goldberg. (129)

Objectives of this work were to determine which of the radionuclides present in sea water could be measured by direct counting of sea water constituents and to measure radionuclide concentrations at various locations along the West Coast. Experiments were designed to measure the radioactivity in particulate material

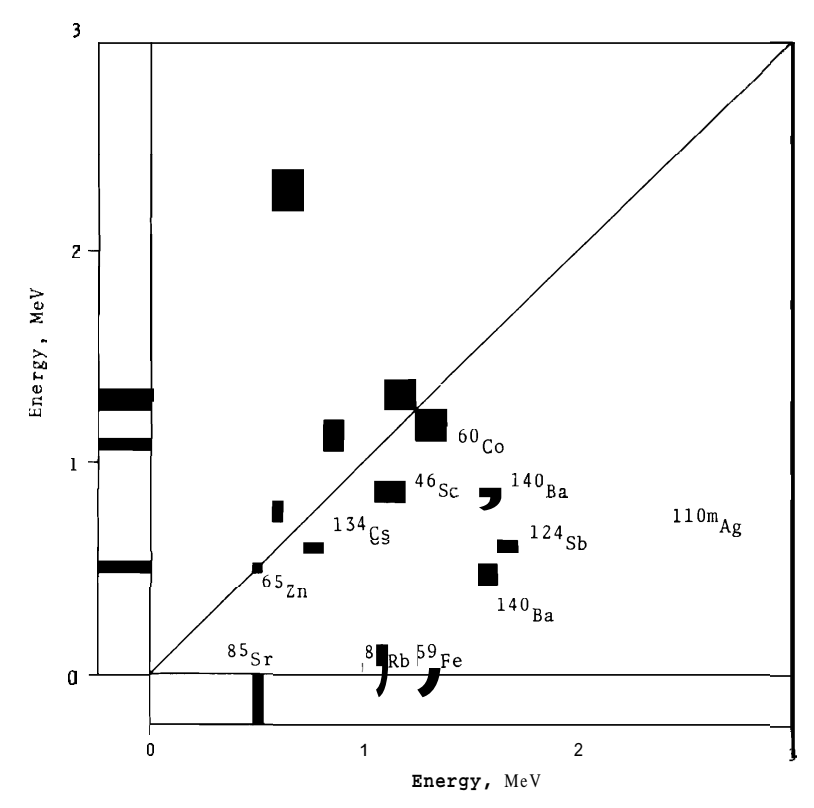

FIGURE 90. Energy Areas Used in the Direct Measurement of Ten Radionuclides in Sea Water 
which could be directly removed by filtration of sea water and to estimate the dissolved radioactivity from the radionuclides retained on an iron oxide or aluminum oxide absorption bed. The radionuclides in these two forms were then compared with those present in marine organisms. One absorption bed for the dissolved radionuclides was steel wool. This was activated by heating overnight in an oven at $600{ }^{\circ} \mathrm{C}$ while passing about 1 liter/min of filtered a ir through the oven. The iron oxide thus formed was crushed and used as a thin bed for radionuclide sorption. The use of iron oxide as an absorbing surface was suggested by the work of Lal, et al. (133) in their method of extracting trace elements from sea water by passing sea water through a matrix containing finely dispersed ferric hydroxide gel. The oxidized steel wool, although a good absorber for removal of several trace elements from sea water, has recently been replaced with aluminum oxide* which serves the same function and is readily available.

In laboratory experiments, the uptake of several radionuclides on columns of oxidized steel wool was measured. Columns 1 am diam were used. A stee 1 wool thickness of about $1 \mathrm{~g} / \mathrm{cm}^{2}$ and a flow rate of $50 \mathrm{~m} 1 / \mathrm{min} / \mathrm{cm}^{2}$ of $\mathrm{sur}-$ face (or the equivalent) was used for the adsorption measurments. The effectiveness of this bed for radionuclide removal was determined by pasing spiked ocean water through a column. The efficiency for the removal of the radionuclides by the oxidized steel

*A Zumina Woe Zm Neutral, AZupharm

Chemicals, New Orleans, Louisiana
TABLE XXIV. Uptake of Soluble Radionuclides from Synthetic Sea Water on Oxidized Steel Wool Column (Flow of $50 \mathrm{ml} / \mathrm{min} \mathrm{cm}^{2}$

\begin{tabular}{c} 
Radionuclide \\
\hline${ }^{46} \mathrm{Sc}$ \\
${ }^{51} \mathrm{Cr}$ \\
${ }^{54} \mathrm{Mn}$ \\
${ }^{59} \mathrm{Fe}$ \\
${ }^{60} \mathrm{Co}$ \\
${ }^{65} \mathrm{Zn}$ \\
$106 \mathrm{Ru}$ \\
${ }^{124} \mathrm{Sb}$ \\
$140 \mathrm{Ba}$ \\
$144 \mathrm{Ce}$
\end{tabular}

\section{$\underline{\text { Percent Retained }}$}

66 $84^{(a)}$ 42 74 62 87 87 49 4.3 47 290

(a) This retention is for chromic ion; the retention of dichromate ion is about $10 \%$.

wool is shown in Table XXIV. Their removal by aluminum oxide is comparable.

During May of 1965 our first extraction of the dissolved trace elements from sea water was made using membrane filtration followed by the oxidized steel wool absorber material. These measurements were made at a point about 10 miles off Coos Bay, Oregon, which is about 200 miles south of the mouth of the Columbia River. Some radionuclides from the Columbia River are present in this area as is true of the entire Oregon coast. Two sea water samples were processed using the large filtration apparatus with about $25 \%$ of it slow passing through the absorption bed. One sample was collected from the surface while the second was pumped from a depth of about $100 \mathrm{ft}$ through a 1.5 in. diam plastic irrigation pipe. The initial flow rates through the combined 8 filters $(0.3 \mu$ pore size) were about $40 \mathrm{ga} 1 / \mathrm{m}$ in but decreased with time during the 30- to 40-minute filtration periods. 
The volume of the surface sample was about 200 gal and that of the depth sample was about 1100 gal. The flow rate through the filtration system during collection of the surface sample decreased to about $1 / 20$ during $30 \mathrm{~min}$ of operation. The flow during collection of the $100 \mathrm{ft}$ depth sample decreased to about $1 / 3$ during 30 min of operation. The filters and the steel wool were dried and counted directly on the multidimensional gamma-ray spectrometer for radionuclide measurement.

Table XXV presents the observed radionuclides in ocean water which are associated with particulate material, and an estimate of the concentrations present in solution. The dissolved radionuclides (which are here defined as those which pass through a $0.3 \mu$ pore size membrane filter) were estimated from the amounts collected on the oxidized steel wool pack and the absorption efficiencies for each radionuclide as shown in Table XXIV. These measurements provided our first information on the concentrations and physical forms of many of the radionuclides in sea water and allowed a comparison of their relative behavior. Where the radionuclides could be measured in both the particulate and dissolved fraction the percentage of particulate was calculated and is included, in the Table. It is interesting that relatively high concentrations of these radionuclides are present at the 100 f t depth. For example, the concentrations of ${ }^{54} \mathrm{Mn},{ }^{144} \mathrm{Ce}$, and ${ }^{106} \mathrm{Ru}$ are present in comparable concentrations at the surface and at the $100 \mathrm{ft}$ depth. On the other hand, the short-lived radionuclide ${ }^{51} \mathrm{Cr}(27 \mathrm{~d})$ is present in the surface water in a concentration of 50 times that at $100 \mathrm{ft}$. The relative zoncentrations and physical forms of these radionuclides at the surface and at $100 \mathrm{ft}$ provide an index of their mixing rates and suggest mechanisms, including normal mixing, sedimentation

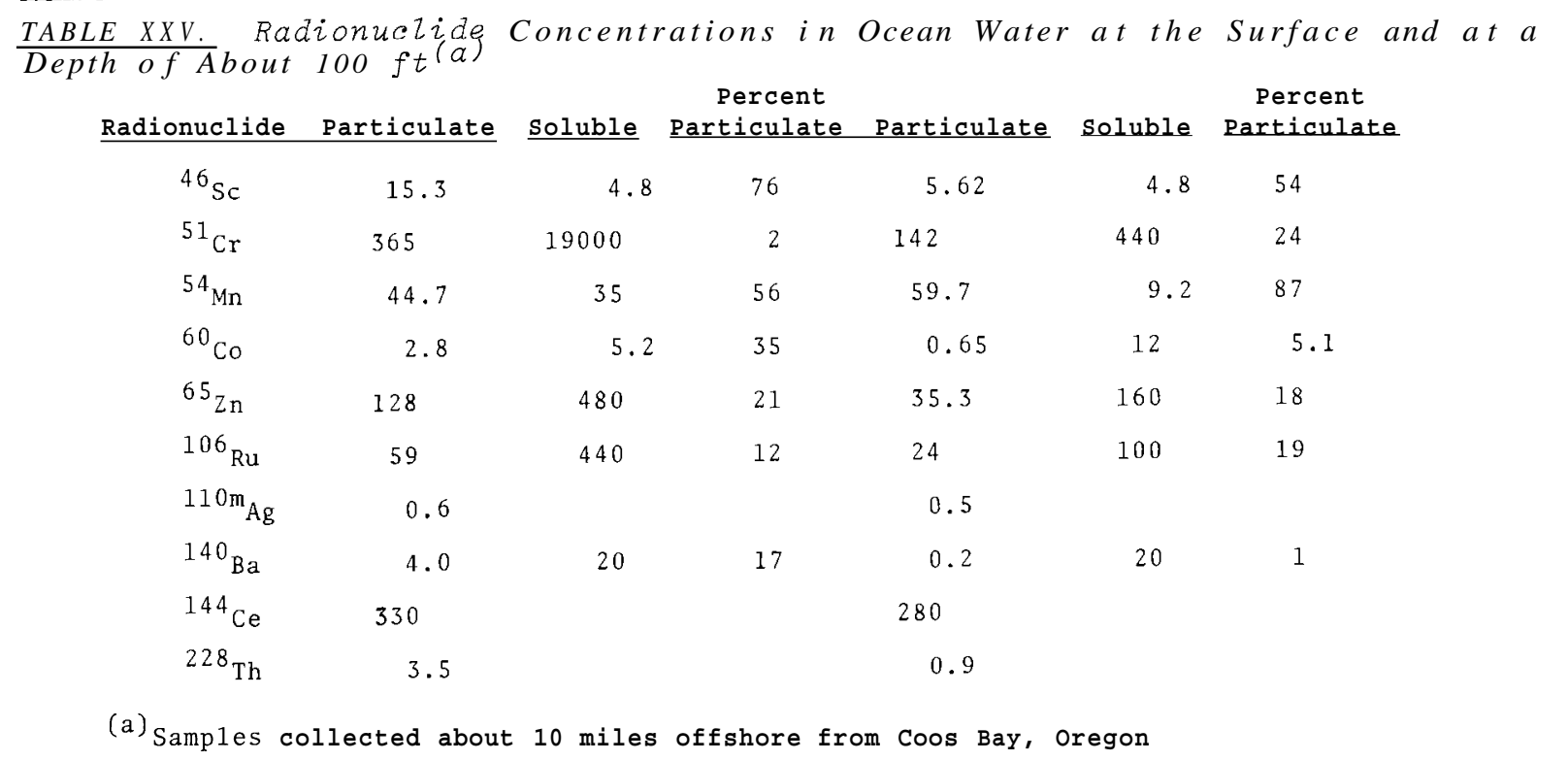


of particulates, and biological participation, whereby the radionuclides reach the deep water.

In Table XXVI the observed radionuclide concentrations in the surface water are compared with those present in a sample of euphausids which we collected (through the courtesy of Dr. Charles Osterberg of Oregon State University) on January 29, 1965, at a point about 35 miles off the Oregon coast mid-way between Astoria and Newport, Oregon. Although these organisms are from a different area, and therefore somewhat different concentrations would be expected, the data serve to indicate the order of magnitude by which the euphausids concentrate the various radionuclides.

TABLE XXVI. Comparison of Radionuclide Concentrations in Ocean Water, Suspended Particulate Material and Marine Organisms from off the Oregon Coast

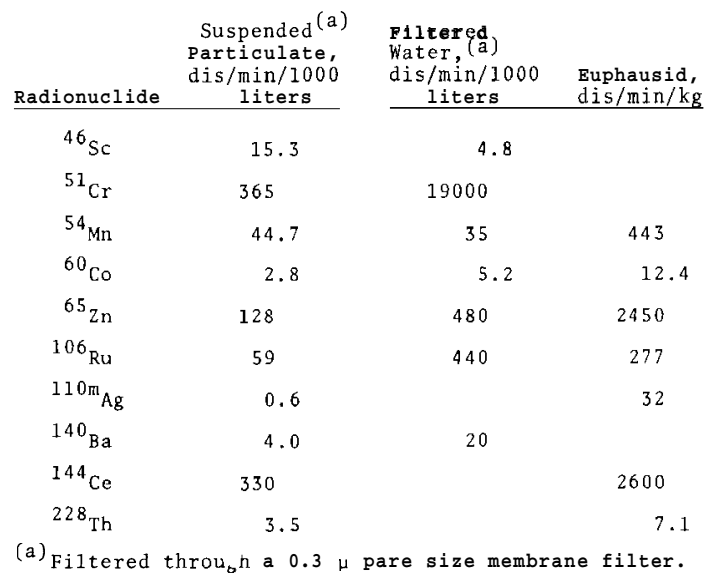

In Table XXVII the physical form of radionuclides in the ocean are compared with those in the Columbia River. It is apparent that these radionuclides are generally more soluble in the ocean
TABLE XXVII. Comparison of Physical Form of Radionuclides in the Ocean with Those in the Columbia River During Low Flow Periods

\begin{tabular}{|c|c|c|}
\hline Radionuclide & $\underset{\text { River }}{\text { Columbia }}(a)$ & $\begin{array}{c}\text { Surface } \\
\text { Ocean Water }\end{array}(b)$ \\
\hline${ }^{46} \mathrm{Sc}$ & 86 & 76 \\
\hline${ }^{51} \mathrm{Cr}$ & 3.9 & 2 \\
\hline${ }^{54} \mathrm{Mn}$ & 90 & 56 \\
\hline${ }^{59} \mathrm{Fe}$ & 83 & -- \\
\hline${ }^{60} \mathrm{Co}$ & 81 & 35 \\
\hline${ }^{65} \mathrm{zn}_{\mathrm{n}}$ & 73 & 21 \\
\hline${ }^{106} \mathrm{Ru}$ & 12 & 12 \\
\hline${ }^{124} \mathrm{Sb}$ & $<1.5$ & $\cdots$ \\
\hline $140_{\mathrm{Ba}}$ & 6.6 & 17 \\
\hline $\begin{array}{l}\text { (a) Columbia } \\
\text { Washingtor } \\
\text { (b) Ocean Wate }\end{array}$ & $\begin{array}{l}\text { er measureme } \\
r, \text { and Noven } \\
\text { s collected }\end{array}$ & $\begin{array}{l}\text { Vancouver, } \\
64 . \\
\text { May, } 1965 .\end{array}$ \\
\hline
\end{tabular}

than in the Columbia River. In Table XXVIII the radionuclide concentrations at the sampling point near Coos Bay, Oregon are compared with those in the Columbia River. The radionuclides in ocean water at Coos Bay are from both fallout and from the Columbia River and the ratios of each radionuclide in

TABLE XXVIII. Comparison of the Absolute and Rezative Concentration of Radionuclides $i n$ the Columbia River with those in the Ocean near Coos Bay, Oregon (Dis/min per 1000 liters)

\begin{tabular}{|c|c|c|c|}
\hline Radionuclide & $\begin{array}{l}\text { Columbia } \\
\text { River Water (a) } \\
\end{array}$ & $\begin{array}{l}\text { Ocean }(b) \\
\text { Water }\end{array}$ & $\begin{array}{c}\text { Ratio: } \\
\text { Columbia River } \\
\text { Ocean }\end{array}$ \\
\hline${ }^{46} \mathrm{Sc}$ & 16,900 & 20.1 & 841 \\
\hline${ }^{51} \mathrm{Cr}$ & $6,330,000$ & 19,400 & 326 \\
\hline${ }^{4} \mathrm{Mn}$ & 4,800 & 79.7 & 60 \\
\hline${ }^{58} \mathrm{Co}$ & 1,000 & & \\
\hline${ }^{59} \mathrm{Fe}$ & 4,820 & & \\
\hline${ }^{60} \mathrm{Co}$ & 1,360 & 8.0 & 170 \\
\hline${ }^{65} \mathrm{zn}_{\mathrm{n}}$ & 82,700 & 608 & 136 \\
\hline $\begin{array}{l}95 \\
{ }^{2} \mathrm{r}- \\
95_{\mathrm{Nb}}\end{array}$ & 3,700 & & \\
\hline${ }^{106}{ }_{\mathrm{Ru}}$ & 8,500 & 499 & 17 \\
\hline${ }^{124} \mathrm{Sb}$ & 4,600 & & \\
\hline${ }^{140} \mathrm{Ba}$ & 8,200 & 24 & 342 \\
\hline
\end{tabular}

(a) Columbia River water at Vancouver during April 1964.

(b) Surface Ocean water 10 miles off Coos Bay, May 1965. 
Columbia River water to that in the ocean serves as an index of its origin. For example, the ratio of the concentrations of ${ }^{46} \mathrm{Sc},{ }^{51} \mathrm{Cr}$, and ${ }^{140} \mathrm{Ba}$ in the Columbia River to that in the ocean is several hundred. This would be expected to be true because of the great dilution which would occur on movement of the Columbia River water radionuclides through 200 miles of ocean water. On the other hand, the ratios of ${ }^{106} \mathrm{Ru}$ and ${ }^{54} \mathrm{Mn}$ are much lower suggesting that fallout is their principal origin. It is realized in making this over-simplified comparison that factors other than dilution may have had major effects on the radionuclide concentrations.
During July, August, and December, 1965, additional ocean water measurements were made to gain a general picture of the levels of radionuclides in ocean water along the West Coast and at distances a few hundred miles offshore. The measurements along the West Coast are summarized in Table XXIX. These measurements which were made during July and August show much lower concentrations of the radionuclides than were observed at Coos Bay during May (see Table XXV) and must. be related to both the flow pattern of Columbia River water in the ocean and the deep water-surface-water exchange in the ocean. The radionuclide concentrations drop off with distance from

TABLE XXIX. Radionuclide Concentrations in Ocean Water AZong the West Coast from the California Border to Torfino, B.C. (a)

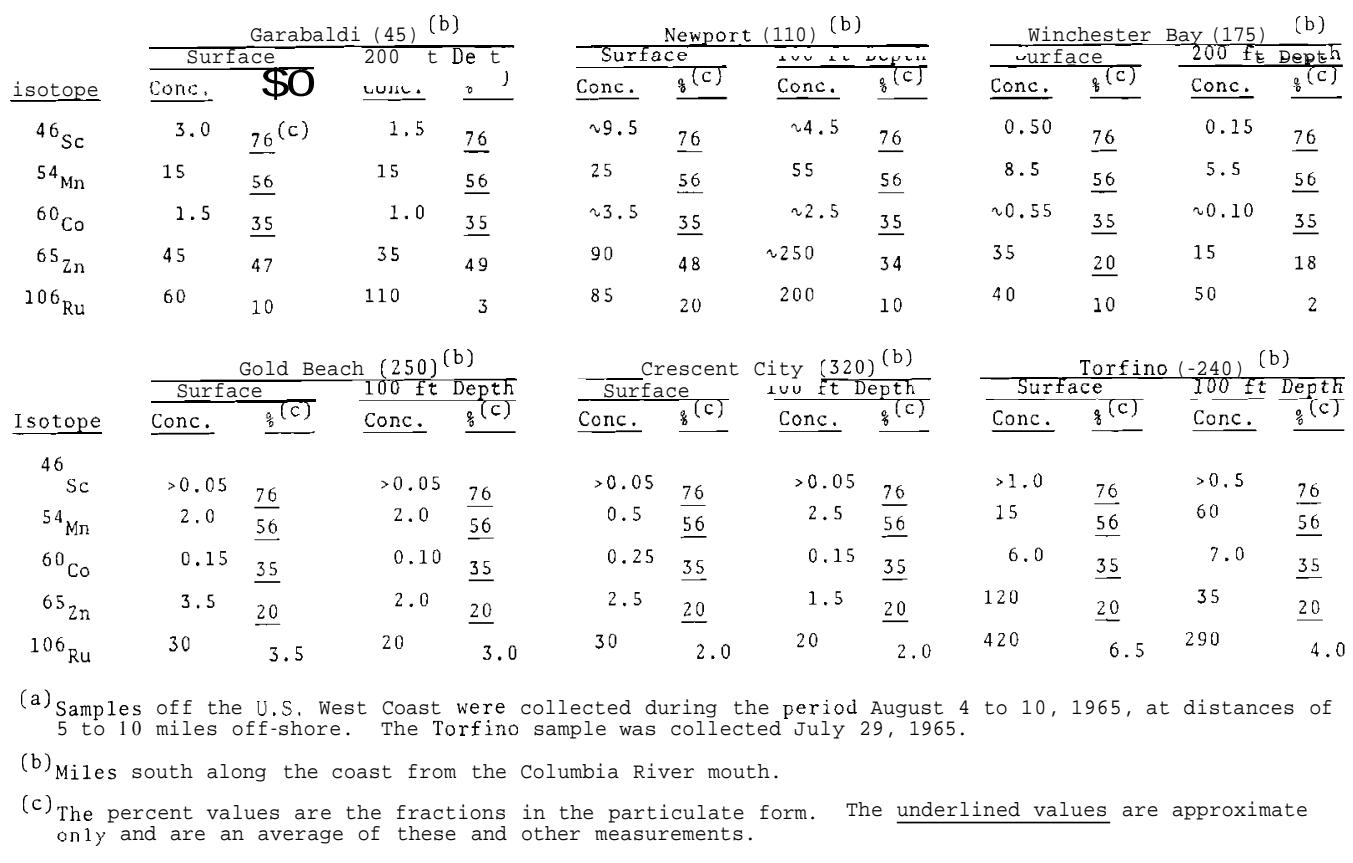


the mouth of the Columbia River in a southerly direction but the ${ }^{65}$ Zn pattern would suggest that the Columbia River radionuclides do reach the California coast. The radionuclide concentrations at Torfino, B.C. were high and their origin, whether Columbia River or fallout, is not clear.

During December, 1965, two of the authors participated as guest scientists on a cruise of the Scripps Jceanographic Institute research - vessel, the Horızon. This cruise covered an area from San Diego due west about 500 miles. Surface and $300 \mathrm{ft}$ depth radionuclide measurements were made at locatons of about 100 and 500 miles west of San Diego and the ob served concentrations are summarized in Table XXX. Radionuclide ${ }^{137}$ Cs was collected on KCF-1 but all other radio nuclides (which passed through the $0.3 \mu$ filters] were collected on aluminum oxide beds. The six artificial radionuclides ${ }^{54} \mathrm{Mn},{ }^{60} \mathrm{Co},{ }^{65} \mathrm{Zn},{ }^{106} \mathrm{Ru},{ }^{137} \mathrm{Cs}$, and ${ }^{144} \mathrm{Ce}$ presumably resulted from nuclear weapons testing while the radionuclides ${ }^{226} \mathrm{Ra},{ }^{228} \mathrm{Th}$, and ${ }^{234} \mathrm{Th}$ are naturally occurring. With the exception of ${ }^{54} \mathrm{Mn}$ and the thorium radionuclides, which are about half in the particulate form, the radionuclides exist mainly in solution. The comparable concentrations of the artificial radionuclides in the surface and deep water samples show that fairly good mixing has occurred in these 10 cations over the top $300 \mathrm{ft}$ of the ocean surface. Further measurement will be required to determine if these observations are typical of the open ocean areas of the world.

TABLEXXX. Radionuclide Concentration in the Ocean (Dis/min per 1000 Ziters, December, 1965 )

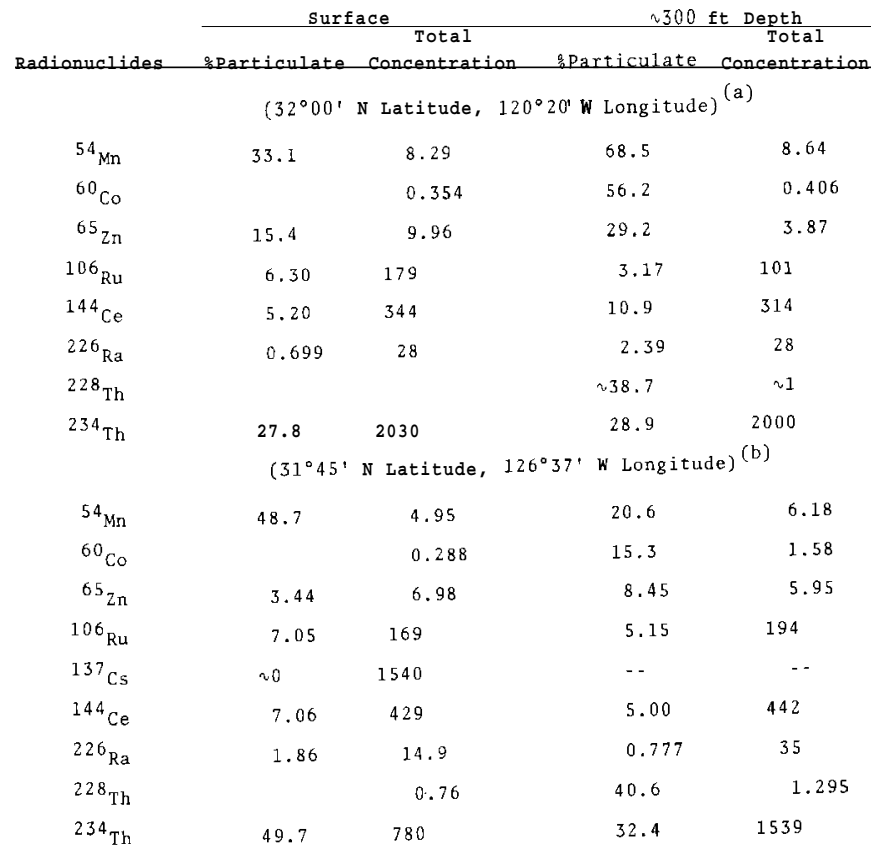

(a) 100 miles West of San Diego

(b) 2500 miles West of San Diego 
COSMIC-RAY PRODUCED RADIONUCLIDES I N THE ENVIRONMENT* - R. W. Perkins and J. M. Nielsen

\begin{abstract}
Radionuclides are continualzy produced by interactions of cosmic rays with the atmosphere, hydrosphere and lithosphere. Most of the studies of these radionuclides are concerned with those produced in the atmosphere or in meteorites. These radionuclides result mainly from spalZation reactions with iron in the case of meteorites; however, ${ }^{14} \mathrm{C} i \mathrm{~s}$ produced by the thermal neutron capture of nitrogen. The production rates of these radionuclides in the atmosphere are strongly dependent or both altitude and geomagnetic latitude. The uses of the radionuclide ${ }^{14} \mathrm{C}$ and tritium in radioactive dating, atmospheric and ocean transport, and other studies are well known. While the radionuclides ${ }^{14} C,{ }^{7}{ }^{B e}$, ${ }_{1} O_{B e}$ and tritium are produced from the light element constituents of the atmosphere, numerous radionuclides are produced by spallation reactions on gtmospheric argon. To date the radionuclides $22 \mathrm{Na}, 24 \mathrm{Na}$,

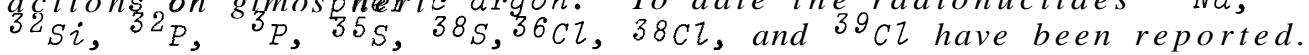
The meteoritic dust which is continually showered into the atmosphere contains spallation products of iron and other cosmic ray induced radionuclides. The concentrations of most of the radionuclides are extremely low and in some cases their artificial production by weapons testing has greatly exceeded their natural production. The biosphere $i s$ continually exposed to the wide spectrum of cosmic-ray produced radionuclides and some of these which are concentrated by biological proaesses can serve as tracers in studies of the biological processes in nature.
\end{abstract}

*Health Physics, Pergamon Press 1965. Vol. 11, pp. 1297-1304.
The trace elements in snow, rain and hail are a mixture of the substances responsible for their original nucleation plus those scrubbed from the atmosphere during the descent to earth. Measurement of the trace elements in precipitation thus provides information on both the nature of the aerosol particles responsible for nucleation and the washout efficiency of precipitation for atmospheric aerosols. A knowledge of the natural concentration of the trace elements provides a basis for selecting trace elements which would be the most sensitive artificial tracers for studying precipitation and atmospheri c dispersion problems. A method has been developed for the direct measurement of the five trace elements silver, zinc, antimony, cobalt, and scandium in snow. It involves the neutron activation of the snow residue to a total exposure of about $10^{19}$ neutrons $/ \mathrm{cm} 2$ followed by direct counting on a multidimensional gamma-ray spectrometer. (98) The sample preparation involves evaporating the snow sample to a small volume, then transferring it quantitatively to a silica glass ampule in which it is dried; the ampule is then sealed for neutron activation. Vycor ware which has been thoroughly leached with boiling dilute $\mathrm{HNO}_{3}$ is used in the evaporation process. The ampules are 
cleaned prior to use by refluxing them overnight in concentrated $\mathrm{HNO}_{3}$. The nitric acid which is used to acidify the melted snow prior to evaporation, and the water which is used along with this $\mathrm{HNO}_{3}$ for rinsing during the transferring process, is purified by a double distillation before use. Following neutron activation, the samples are held for a 2 to 4 week period to allow the ${ }^{24} \mathrm{Na}$ and other short-lived radionuclides to decay. The ampules are then opened and the samples quantitatively transferred to standard counting dishes. The trace elements silver, zinc, antimony, cobalt, and scandium are determined from their daughter radionuclides ${ }^{110 m_{\mathrm{Ag}},}{ }^{65} \mathrm{zn},{ }^{124} \mathrm{Sb},{ }^{60} \mathrm{Co}$, and ${ }^{46} \mathrm{Sc}$, respectively.

The radionuclide mixture is counted on a multidimensional gamma-ray spectrometer, and the radionuclide concentrations are determined from the coin- cidence counting rates of their characteristic gamma rays. Typical concentrations of the trace elements which have been observed in fresh snow samples are presented in Table XXXI.

The concentrations of the trace elements cobalt, scandium, antimony, and silver are extremely low, and so these trace elements have been selected for use as tracers in studying atmospheric precipitation processes.

TABLE XXXI. Trace Element Concentrationsin Typical Snow Samples $\underline{\text { Trace Element }}$

Zinc

Cobalt

Scandium

Antimony

Silver
Concentration, ppb

4

0.04

0.01

0.1

CONCENTRATION OF FALLOUT RADIONUCLIDES DURING WET AND DRY SEASONS-C. W. ThOmas

The concentrations of the fallout radionuclias ${ }^{106} \mathrm{Ru},{ }^{88} \mathrm{Y},{ }^{60} \mathrm{Co} .{ }^{54} \mathrm{Mn}$. ${ }^{22} \mathrm{Na},{ }^{7} \mathrm{Be}, 95_{\mathrm{Zr}-} 95_{\mathrm{Nb}}, 1 \dot{2}_{4} \mathrm{Sb},{ }_{2} 5_{\mathrm{Sb}}$, $134 \mathrm{Cs},{ }^{137} \mathrm{Cs},{ }^{140} \mathrm{Ba}-{ }^{140} \mathrm{La}$, and ${ }^{144} \mathrm{Ce}$ were determined in samples of rain and in composite samples of rain and dry fallout. Both kinds of samples were collected 15 miles northwest of Richland, Washington, on top of Rattlesnake Mountain, elevation 3400 $\mathrm{ft}$, over the period 10-1-64 to 9-30-65 Rain trays were designed so that the water could be automatically removed by draining directly into large plastic containers or could be passed first through a filter, then through two ion exchange columns in series before going to the large effluent container. Under normal conditions (i.e., absence of blowing dust and falling rain together) the rain samples passed through the ion-exchange columns with approximately a $10 \mathrm{~min}$ delay period. During dry periods, rain trays were prevented from being able to later deliver any dry fallout which they may have caught by spraying them with Krylon plastic every day over sides and bottom of the trays to fix the particles. The composite 
samples (dry fallout and rain fallout mixed) were collected in 15-day increments using similar large plastic trays but containing $1 / 4$ in. of water. After collection, these composite or "total deposition" samples were evaporated to dryness in the laboratory, the salts suspended in an agar-agar gel and the radionuclide concentrations determined directly by multidimensional gamma-ray spectrometry. A standard U. S. Weather Bureau Rain Gage was used to measure the amount of precipitation.

The average rainfall in this area is only 7 to $8 \mathrm{in./yr}$ and $\mathrm{rainfall}$ is not the predominant mechanism for fallout deposition. During the period covered by this report weather conditions were unusual for this area; during the winter of 1964-1965 the greatest snowfall in 53 years was recorded, and this made it impossible to attend the trays during the period 12-2-64 to 3-1-65, However, precipitation for 1965 was the lowest recorded for 36 years and this meant longer than normal dry periods. The total precipitation measured at the sampling site for the period 3-1-65 to
9-30-65 was 2.04 in., of which 0.72 in. fell in one $5 \mathrm{hr}$ period.

It was reported previously(135) that various radionuclides show definite anionic, zationic, and neutral tendencies as indicated by their removal in the anion or the cation exchange column or by neither. However, it was pointed out that the reported results might reflect merely the filtering action of the leading (anion) cartridge on a large particle holding the radionuclides. To confirm such a filtering effect, if any, of the leading cartridge, several samples were collected with the cation cartridge in the leading position. The results indicated no filtering action by the leading cartridge.

The most noticeable difference compared to the previous work was the larger percentage of insolubles as determined by removal on the filter preceding the ion exchange columns. This increase could be due to the excessively dry season resulting in larger amounts of particulates in the air. The results of the present work are given in Table XXXII .

TABLE XXXII. Radionuclides in Rainwater Fractions

\begin{tabular}{|c|c|c|c|c|}
\hline \multirow{2}{*}{ I sotope } & \multirow{2}{*}{$5 \mu$ Filter } & \multicolumn{2}{|c|}{$\%$ of Total Activity } & \multirow{2}{*}{$\underline{\text { Effluent }}$} \\
\hline & & Cation Resin & Anion Resin & \\
\hline $124 \mathrm{Sb}$ & 33 & 14 & 40 & 13 \\
\hline${ }^{125} \mathrm{Sb}$ & 24 & 11 & 50 & 14 \\
\hline${ }^{95} \mathrm{Zr}-{ }^{95_{\mathrm{Nb}}}$ & 38 & 5 & 42 & 16 \\
\hline${ }^{106} \mathrm{Ru}$ & 13 & 15 & 50 & 22 \\
\hline${ }^{60} \mathrm{Co}$ & 25 & 33 & 9 & 33 \\
\hline${ }^{22} \mathrm{Na}$ & 1 & 73 & 4 & 22 \\
\hline${ }^{54} \mathrm{Mn}$ & 14 & 73 & 4 & 6 \\
\hline${ }^{140} \mathrm{Ba}-{ }^{140} \mathrm{La}$ & 17 & 57 & 9 & 13 \\
\hline${ }^{7} \mathrm{Be}$ & 15 & 66 & 11 & 9 \\
\hline${ }^{134} \mathrm{Cs}$ & 36 & 41 & 15 & 8 \\
\hline${ }^{88} Y$ & 49 & 19 & 22 & 10 \\
\hline${ }^{137} \mathrm{Cs}$ & 58 & 35 & 3 & 5 \\
\hline${ }^{144} \mathrm{Ce}$ & 70 & 7 & 12 & 8 \\
\hline
\end{tabular}


Most of the ${ }^{106} \mathrm{Ru},{ }^{124} \mathrm{Sb}$, and ${ }^{125} \mathrm{Sb}$ collected on the anion column, indicating anionic tendencies for these nuclides. The radionuclides ${ }^{22} \mathrm{Na},{ }^{54} \mathrm{Mn}$, ${ }^{140} \mathrm{Ba}-{ }^{140} \mathrm{La}$, and ${ }^{7} \mathrm{Be}$ showed strong cationic tendencies while ${ }^{88} \mathrm{Y},{ }^{137} \mathrm{Cs}$, and ${ }^{144} \mathrm{Ce}$ showed their highest percentage in insoluble material. In general these data agree with those of the previous measurements.

During the collection period two Chinese nuclear detonations added short half-life radionuclides to the atmosphere and the concentrations are reflected by the data given here.

Table XXXIII shows the cumulative composite deposition as well as the cumulative deposition due to rainfall.

Certain observations on the deposition are of interest. The large ratio noted for ${ }^{124} \mathrm{Sb}(60 \mathrm{~d})$ is due mainly to a large deposition of unexplained origin occurring around the first of April,
1965; this represented $80 \%$ of the total ${ }^{124}$ Sb deposition for the period. A high concentration of ${ }^{124}$ Sb was also noted by air sample measurements at that time. A large deposition of ${ }^{60}$ Co representing $60 \%$ of the total occurred in August, 1965; however, unlike the ${ }^{124} \mathrm{Sb}$ air sample, the air sample measurements did not show any abnormal ${ }^{60}$ Co concentrations during the period. Also from the table it appears that the cosmicray spallation products ${ }^{7} \mathrm{Be}$ and ${ }^{22} \mathrm{Na}$ are carried down largely by the rain. In general, the radionuclides that show high tendencies toward the particulate condition, such as ${ }^{137} \mathrm{Cs}$ and ${ }^{144} \mathrm{Ce}$, can deposit more efficiently during dry periods.

Since there appeared to be a selectivity in deposition during wet and dry periods, a comparison was made of the deposition of two of the most divergent radionuclides. The two ra-

TABLE XXXIII. Cumulative Ground Deposition of 13 Fallout Radionuclides Measured at Richland, Washington (a)

\begin{tabular}{|c|c|c|c|}
\hline Isotope & $\begin{array}{r}\text { Composite } \\
\text { dis/min/ft } \\
\end{array}$ & $\begin{array}{c}\begin{array}{r}\operatorname{Rain}, \\
\mathrm{dis} / \mathrm{min} / \mathrm{ft}^{2}\end{array} \\
\end{array}$ & Composite/Rain \\
\hline${ }^{7} \mathrm{Be}$ & 2220 & 1400 & 1.58 \\
\hline${ }^{22} \mathrm{Na}$ & 1.54 & 0.79 & 1.95 \\
\hline${ }^{54} \mathrm{Mn}$ & 157 & 76 & 2.06 \\
\hline${ }^{60} \mathrm{Co}$ & 21.2 & 1.75 & 12.1 \\
\hline${ }^{88} \mathrm{Y}$ & 0.91 & 0.41 & 2.22 \\
\hline${ }^{95} \mathrm{Zr}^{-95} \mathrm{Nb}$ & 1872 & 693 & 2.70 \\
\hline${ }^{106} \mathrm{Ru}$ & 803 & 366 & 2.19 \\
\hline${ }^{124} \mathrm{Sb}$ & 21.8 & 1.76 & 12.4 \\
\hline${ }^{125} \mathrm{Sb}$ & 353 & 167 & 2.13 \\
\hline${ }^{134} \mathrm{Cs}$ & 2.5 & 0.35 & 7.14 \\
\hline${ }^{137} \mathrm{Cs}$ & 565 & 121 & 4.67 \\
\hline $140 \mathrm{Ba}-{ }^{140} \mathrm{La}$ & 1383 & 772 & 1.79 \\
\hline${ }^{144} \mathrm{Ce}$ & 1448 & 451 & 3.21 \\
\hline
\end{tabular}


dionuclides ${ }^{137} \mathrm{Cs}$, highly particulate but cationic, and ${ }^{106} \mathrm{Ru}$, associated only slightly with particulate and high anionic, were chosen. The ratios in which these two isotopes occurred in air, rainfall, and in total deposition are presented in Table XXXIV. The data indicate that some mechanism for selectivity does exist during wet and dry seasons at least between these two radionuclides.

TABLE XXXIV. Concentration Ratio, ${ }^{106}$ Ru to ${ }^{137}$ Cs, in Rainfall, Total Deposition and Air

\begin{tabular}{|c|c|c|c|}
\hline \multirow[b]{2}{*}{$\underline{\text { Period }}$} & \multirow[b]{2}{*}{$\underline{\text { Rainfall }}$} & \multicolumn{2}{|c|}{${ }^{106} \mathrm{Ru} /{ }^{137} \mathrm{Cs}$} \\
\hline & & Composite & Air \\
\hline $\begin{array}{l}10-15-64 \\
10-28-64\end{array}$ & 3.4 & 2.3 & 2.0 \\
\hline $\begin{array}{l}3-4-65 \\
3-15-65\end{array}$ & (a) & 1.2 & 1.8 \\
\hline $\begin{array}{l}3-15-65 \\
3-31-65\end{array}$ & (a) & 1.6 & n.d. (b) \\
\hline $\begin{array}{l}3-31-65 \\
4-15-65\end{array}$ & 3.8 & 1.5 & 1.9 \\
\hline $\begin{array}{l}4-15-65 \\
4-30-65\end{array}$ & 1.8 & 1.5 & n.d. \\
\hline $\begin{array}{l}4-30-65 \\
5-14-65\end{array}$ & (a) & 1.6 & 1.7 \\
\hline $\begin{array}{l}5-14-65 \\
6-1-65\end{array}$ & 3.2 & 1.3 & 1.8 \\
\hline $\begin{array}{l}6-1-65 \\
6-15-65\end{array}$ & 5.8 & 1.6 & n.d. \\
\hline $\begin{array}{l}6-15-65 \\
6-30-65\end{array}$ & 3.8 & 1.2 & 1.7 \\
\hline $\begin{array}{l}7-16-65 \\
7-30-65\end{array}$ & 5.3 & 1.1 & 1.4 \\
\hline $\begin{array}{l}7-30-65 \\
8-16-65\end{array}$ & 3.4 & 1.2 & 1.4 \\
\hline $\begin{array}{l}8-16-65 \\
8-30-65\end{array}$ & 10.0 & 1.1 & 1.2 \\
\hline $\begin{array}{l}8-30-65 \\
9-15-65\end{array}$ & 5.6 & 1.8 & 1.3 \\
\hline $\begin{array}{l}9-15-65 \\
10-1-65\end{array}$ & 7.1 & 1.8 & 2.7 \\
\hline
\end{tabular}

(a) No precipitation occurred during this period.

(b) $\mathrm{n} . \mathrm{d} .=$ not determined. 
AIRBORNE RADIONUCLIDE MEASUREMENTS-R. W. Perkins

In 1962 a program was started to study the behavior of airborne radionuclides and to develop direct counting methods for their measurement. A summary which included the concentrations of 13 airborne radionuclides at Richland, Washington, was published in the Proceedings of the Second Conference on "Radioactive Fallout from

- Nuclear Weapons Tests." These measure ments covered the time period 1962 through September, 1964. The studies have continued and the number of airborne radionuclides measured has been extended to include 18 natural and artificial radionuclides. In addition a sampling station has been added at Point Barrow, Alaska, and one is being set up at Rio de Janeiro, Brazil.

Since discontinuance of the large scale atmospheric nuclear tests in December, 1962, the air concentrations of most of the artificial radionuclides have dropped by one or two orders of magnitude or more. To maintain a high sensitivity for radionuclide measurement the original $100 \mathrm{ft}^{3} / \mathrm{min}$ air sampling pump a t Richland, Washington, was replaced with a $700 \mathrm{ft}^{3} /$ min pump in February, 1965. In addition, a larger multidimensional gamma-ray spectrometer detector system, using an $11 \mathrm{in}$. diam by 6 in. thick detector has been built and will be in use shortly. This detector system will improve the detection sensitivity for several of the radionuclides by three or fourfold. With the drop in concentrations of the nuclear weapons-producedradionuclides those radionuclides which are released in minute amounts from the Hanford plants have become evident. Unfortunately some of the radionuclides so released are the same as those being monitored in fallout, and this has caused some problems in interpreting the nuclear weapons fallout contribution.

Tables XXXV and XXXVI present the airborne radionuclide concentrations at Richland, Washington, and Point Barrow, Alaska, respectively. The Point Barrow samples are, of course, not affected by the Hanford plant and should be representative of airborne radionuclide concentrations at that latitude.

Table XXXV includes the air concentrations of 18 airborne radionuclides at a sampling point 6 miles north of Richland, Washington. All radionuclides listed were measured by direct counting on a multidimensional gamma-ray spectrometer. The radionuclides ${ }^{7} \mathrm{Be}$ and ${ }^{228}$ Th are naturally occurring while the others have resulted from nuclear testing or from the nuclear plant operation. The ${ }^{22} \mathrm{Na}$ is produced by cosmic ray spallation as well as by nuclear weapons testing. There is no significant contribution to the ${ }^{22} \mathrm{Na},{ }^{88} \mathrm{Y}$, and $110 \mathrm{~m} A g$ from the Hanford plant and the contribution to all others except ${ }^{46} \mathrm{Sc},{ }^{134} \mathrm{Cs}$, and perhaps ${ }^{60} \mathrm{Co}$ and ${ }^{65} \mathrm{Zn}$ is usually very small. The ${ }^{46}$ Sc is due entirely to the Hanford plant operation. 
TABLE XXXV. Radionuclide Concentrations in the Air near Richland, Washington During 1964 - 1965, Dis/min per $10^{6} \mathrm{ft}^{3}$ (S.T.P.)

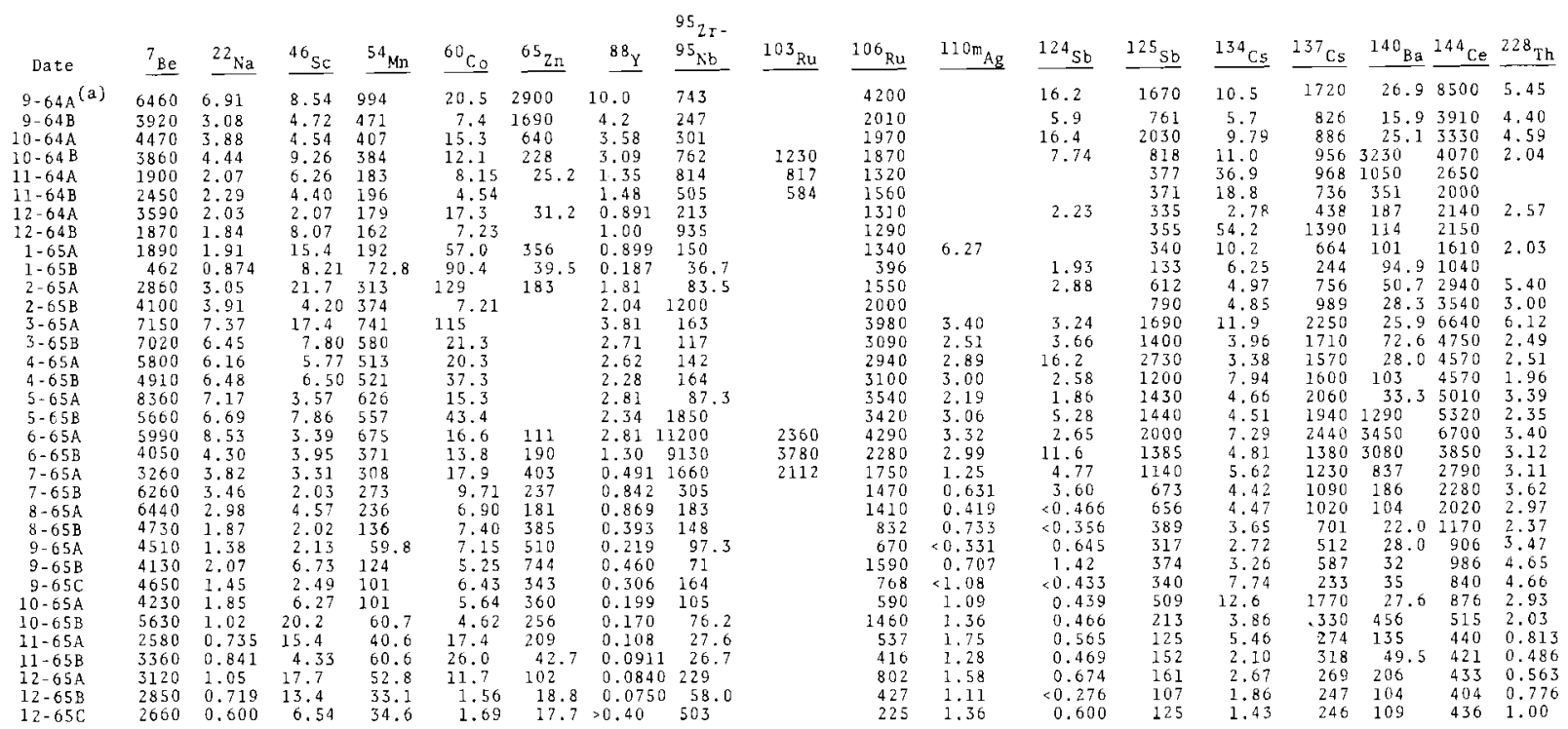

${ }^{(a)} \mathrm{A}, \mathrm{B}$, and $\mathrm{C}$ indicate successive fractions of the month

TABLE XXXVI. Radionuclide Concentrations in Air at Point Barrow, Alaska, During $1964-1965$

\begin{tabular}{|c|c|c|c|c|c|c|c|c|c|c|c|c|c|c|}
\hline Date & ${ }^{7} \mathrm{Be}$ & ${ }^{22} \mathrm{Na}$ & ${ }^{54} \mathrm{Mn}$ & ${ }^{60} \mathrm{Co}$ & ${ }^{88_{Y}}$ & $\begin{array}{l}95 \mathrm{Zr}- \\
95_{\mathrm{Nb}}\end{array}$ & ${ }^{103^{\mathrm{Ru}}}$ & ${ }^{106} \mathrm{Ru}$ & $124 \mathrm{Sb}$ & ${ }^{125} \mathrm{Sb}$ & ${ }^{134} \mathrm{Cs}$ & ${ }^{137} \mathrm{Cs}$ & ${ }^{140} \mathrm{Ba}$ & ${ }^{144} \mathrm{Ce}$ \\
\hline $11-64$ & 3770 & 3.52 & 379 & 5.14 & 2.72 & 720 & & 1740 & $\times 2.0$ & 695 & $\leadsto 1.2$ & 798 & 519 & 3560 \\
\hline $12-64$ & 2010 & 2.26 & 190 & 2.08 & 1.4 & 168 & & 885 & 1.7 & 355 & $\sim 0.6$ & 423 & 54.7 & 1850 \\
\hline $1-65$ & 2570 & 2.62 & 240 & 3.10 & 1.3 & 78.9 & & 1090 & $<0.9$ & 464 & $\sim 0.8$ & 549 & $<9$ & 2290 \\
\hline $2-65$ & 3270 & 3.77 & 311 & 4.36 & 1.8 & 47.1 & & 1280 & $<0.8$ & 629 & $\sim 1.1$ & 775 & 143 & 2720 \\
\hline $3-65$ & 3040 & 3.61 & 291 & 3.76 & 1.4 & 29.5 & & 1370 & $<0.7$ & 607 & $w 1.2$ & 749 & 43 & 2610 \\
\hline $4-65$ & 2690 & 2.96 & 268 & 4.09 & 1.3 & 21.8 & & 1410 & $\approx 0.7$ & 594 & 20.6 & 765 & $<I$ & 2470 \\
\hline $5-65$ & 1570 & 1.67 & $14 \mathrm{I}$ & 2.23 & 0.74 & 45.3 & 59 & 876 & & 306 & $\approx 0.5$ & 410 & 108 & 1290 \\
\hline 6.65 & 1930 & 2.15 & 145 & 2.47 & 0.55 & 659 & 351 & 1070 & & 343 & 20.7 & 455 & 403 & 1210 \\
\hline
\end{tabular}

It is planned to publish a complete review of the fallout measurements later this year and only a few of the more obvious observations will be considered here.

The air concentrations at point Barrow are generally about one-half of those at Richland. The ${ }^{7}$ Be shows a season variation quite similar to the long-lived fission products thus indicating the importance of the stratospheric contribution to the spring and summer tropospheric concentrations. The ${ }^{22} \mathrm{Na}$ concentrations during 1965 were one-fifth to one-tenth of those observed during 1963 thus confirming the origin of the majority of ${ }^{22} \mathrm{Na}$ as produced by nuclear weapons rather than by 
cosmic rays. The ${ }^{88} \mathrm{Y}$ and ${ }^{124} \mathrm{Sb}$ which were introduced as tracers in a November and December, 1962 nuclear test ${ }^{(136)}$ are near the detection limit, but will probably be measurable during part of 1966. While the ${ }^{134} \mathrm{Cs}$ to ${ }^{137} \mathrm{Cs}$ ratio at Point Barrow, Alaska, is about 1:1000 it is about $1: 100$ at Rich- land, Washington, which suggests that the major part of the ${ }^{134} \mathrm{Cs}$ may have originated from the Hanford plant. The Chinese nuclear tests produced major increases in the ${ }^{140} \mathrm{Ba},{ }^{95} \mathrm{Zr}-{ }^{95} \mathrm{Nb}$, and ${ }^{103} \mathrm{Ru}$ (and short-lived radionuclides not included here) (137), as well as detectable increases in most of the long-lived radionuclides.

133 Xe AS AN ATMOSPHERIC TRACER-J. D. Ludwick

${ }^{133}$ Xe was emitted as a gas from a pressure cylinder under controlled conditions along the Hanford Meteorological grid. The purpose of these experiments was to measure the downwind radioxenon concentrations and interpret the results in terms of an atmospheric diffusion model. (138) Additionally, two particulate tracers, zinc sulfide and fluorescein (sodium salt) were simultaneously dispersed from separate individual generators. Field collection of the particulates was made with many samplers over a wide area, but the 25 xenon samplers were selectively located, depending upon atmospheric conditions at the time of the release. Rapid zinc sulfide fluorescence determinations were conducted for those particulate samples collected at xenon sampler locations. This helped to determine the significant radioxenon activity levels to be expected. In this manner, laboratory ${ }^{133}$ Xe determination was programmed to eliminate any possible contamination from earlier samples. The approximate ${ }^{133}$ Xe concentrations were also used to determine which samples required special purification of the xenon to remove the last traces of other radioisotopes such as radon. Samples in which the radioimpurity level was expected to be greater than $1 \%$ of the total activity were subjected to chromatographic separation over charcoal.

One of the most significant developments from the xenon field experiments is the ability to compare the theoretical models for diffusion in the atmosphere with the behavior actually observed. Additionally, the diffusion of xenon, where no interaction with surfaces or vegetation is expected, can be compared with the diffusion of simultaneously released tracers which do react with or deposit on surfaces. Such comparisons can aid in improving the understanding of deposition mechanisms and lead to establishing dispersion models adequately accounting for them.

Figure $91^{(139)}$ illustrates a typical theoretical model of downwind exposure where $\mathrm{V}$ is the deposition 
velocity and $\bar{u}_{0}$ is the average wind speed at ground level. Notice that the curve representing a zero deposition velocity should indicate a gas such as xenon, while material which deposits will behave in accord with one of many curves depending upon the deposition value. Thus, the farther a depositing material is from its source, the more depletion will have

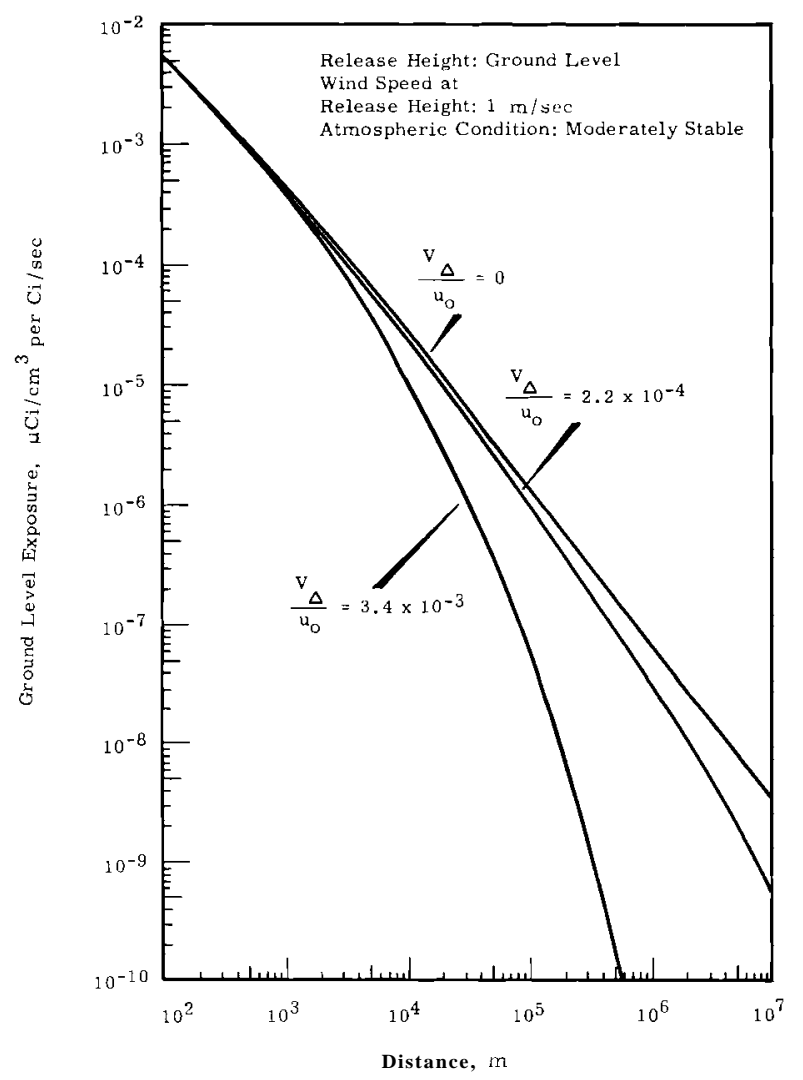

FIGURE 91. Expected Ground Leve 2 Exposure taken place and the less of the material remains in the atmosphere. Illustration of this point is shown on Figures 92, 93, and 94, showing results from the last three field tests comparing ${ }^{133}$ Xe and zinc sulfide tracers.

At the present time, fluorescein determinations are not complete; however, a comparison of simultaneous zinc sulfide and fluorescein emissions is given elsewhere. (140)

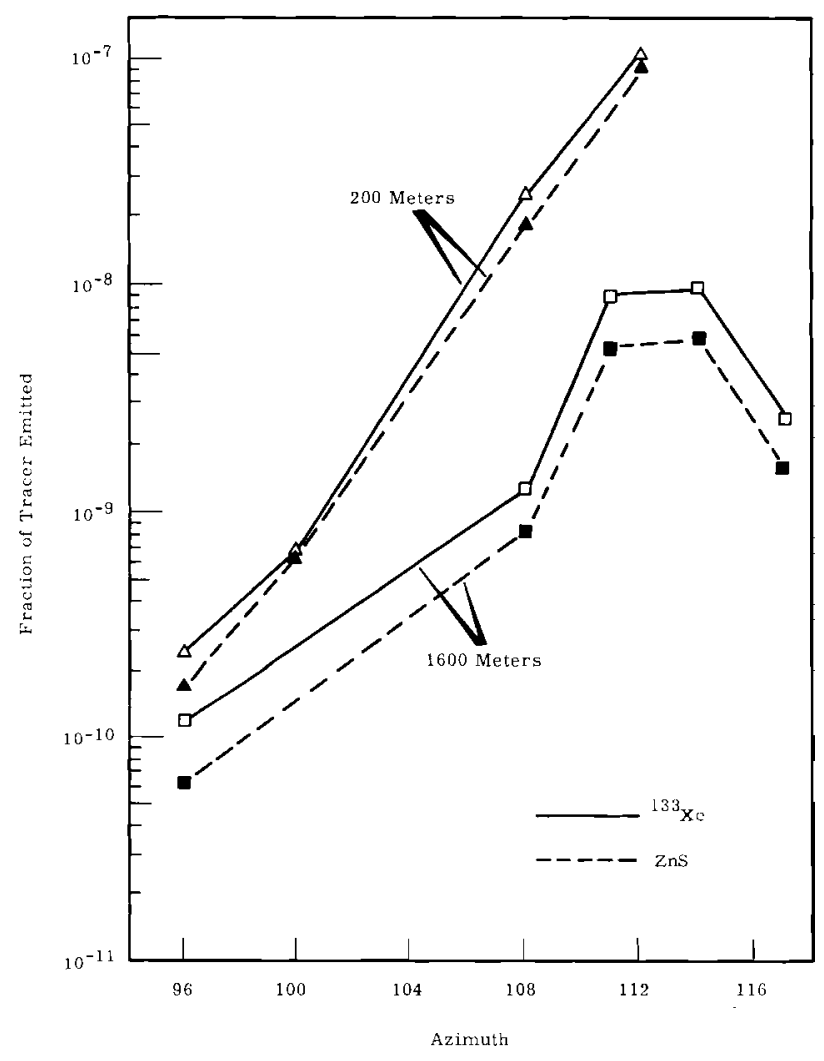

FIGURE 92. ${ }^{133}$ Xe Multitracer Run No. 3 


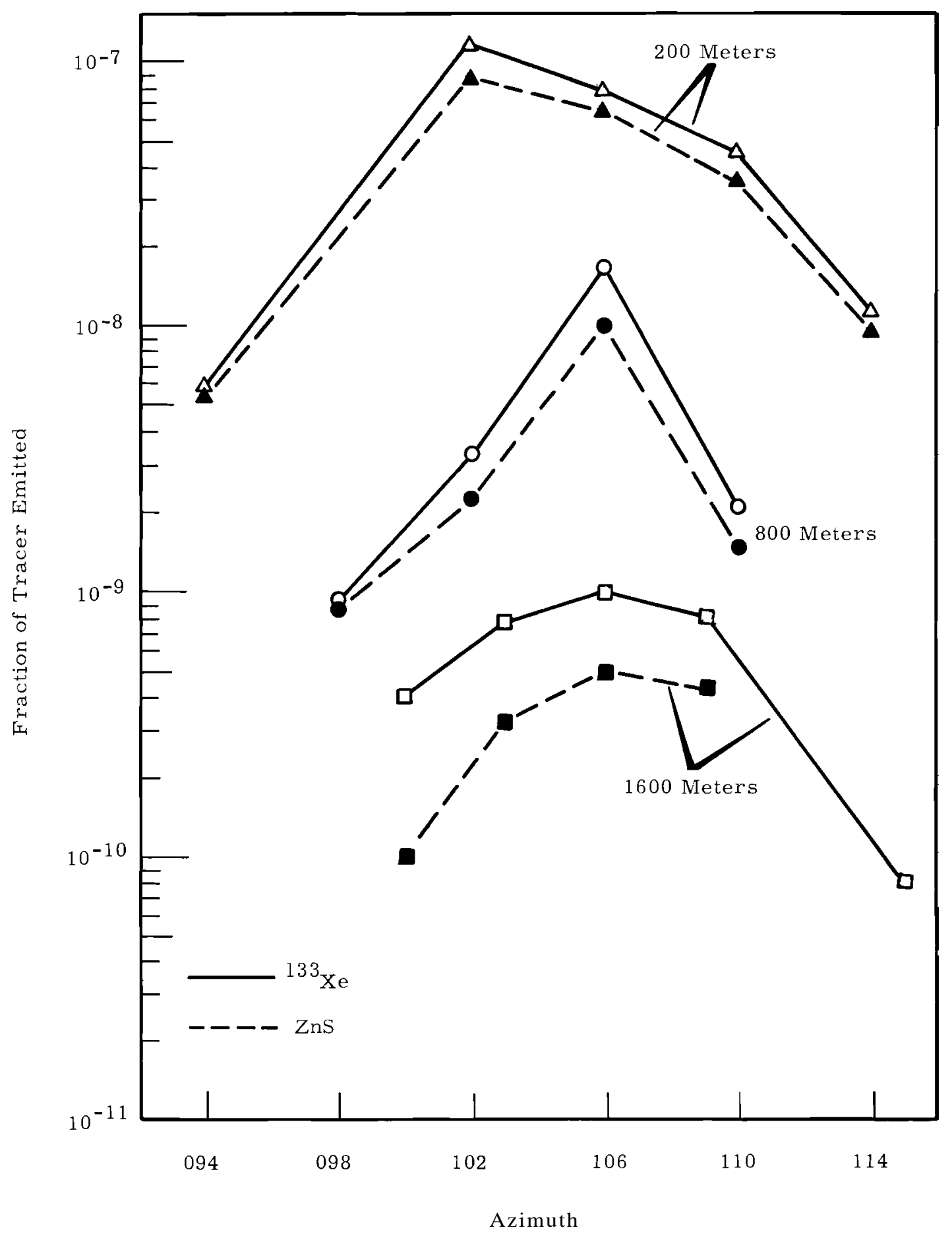

FIGURE 93. $\quad{ }^{133}$ Xe MuZtitracer Run No. 4 


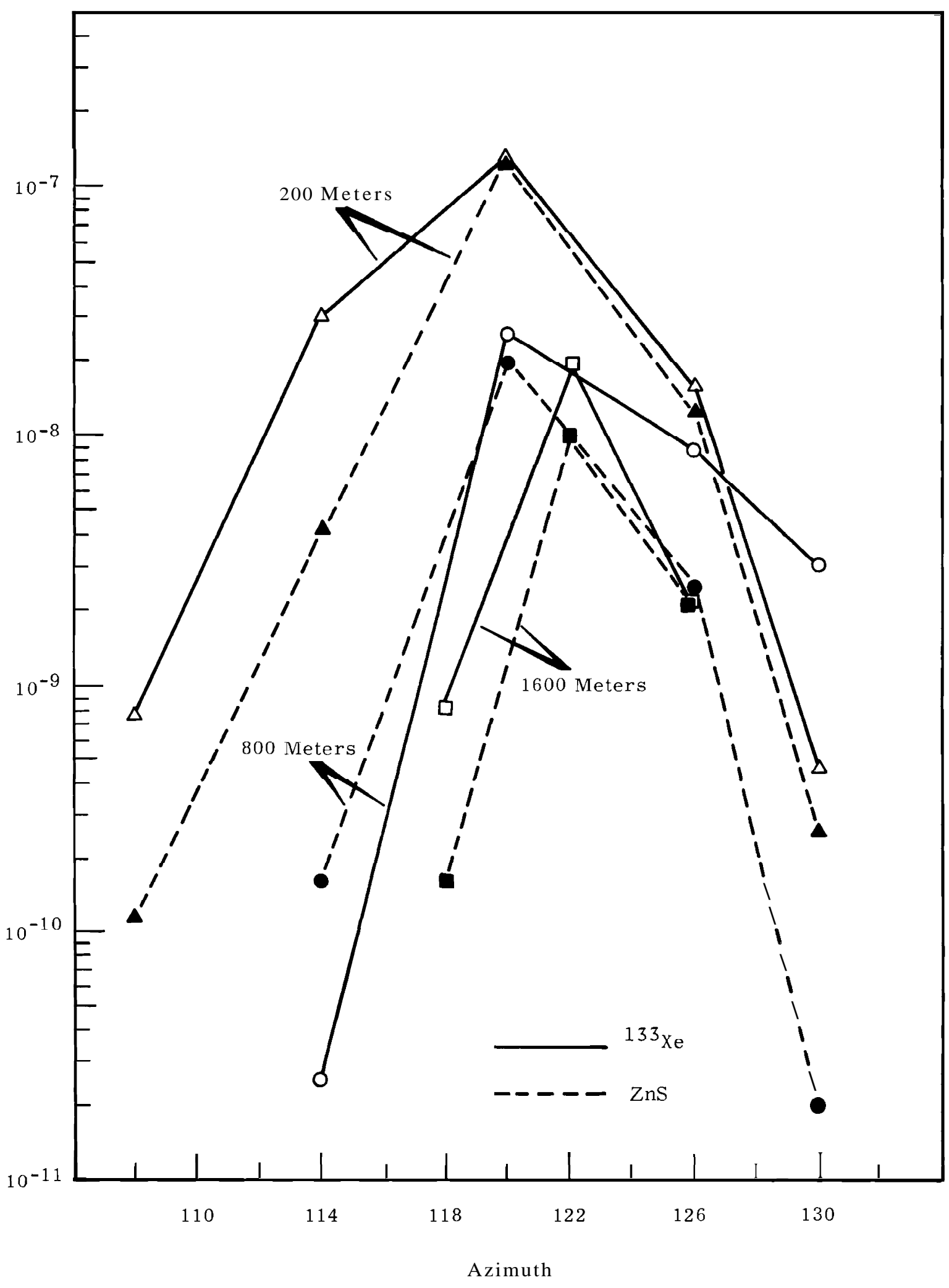

FIGURE 94. ${ }^{133}$ Xe Multitracer Run No. 5 
THE MEASUREMENT OF ${ }^{131}$ I TRANSPORT IN ATMOSPHERIC DIFFUSION EXPERIMENTSJ. D. Ludwick

In the late summer of 1963 a controlled ${ }^{131}$ I release was made at Hanford. (141) The experiment was conducted under slightly stable at mospheric conditions just at daybreak. Extensive studies of ground and vegetation deposition were made at that time in addition to a complete wind diffusion analysis. The results of several phases of the problem indicated a need for further study. For example, a large change in the form of the iodine was observed after only a brief downwind travel. This was evidenced, in part, by an increasingly large iodine fraction in the particulate phase. Some anomalous differences were observed between results from air sampling stations $1.5 \mathrm{~m}$ above ground level and those on towers at the same geographic position. A larger emmission was needed to measure more accurately the trace iodine concentrations at great distances.

Iodine Run No. 2

On the morning of August 24, 1965, a release of about $0.4 \mathrm{Ci}$ of ${ }^{131} \mathrm{I}$ was made. At the $800 \mathrm{~m}$ distance a wooden structure had been erected with horizontal crossmembers from which hung samples of basic building materials and surfaces. These had typical surfaces which a cloud of emitted iodine would probably contact in an urban area in any accidental ${ }^{13} I_{I}$ release. The amounts of deposition obtained on the various surfaces are shown in Table XXXVII. The "area exposed" in each case is the planar area and not the actual

\begin{tabular}{|c|c|c|}
\hline \multicolumn{3}{|c|}{$\begin{array}{l}\text { TABLE XXXVII. Comparative Deposition of } \\
\text { IJII on Building Material Surfaces }\end{array}$} \\
\hline Surface & Area Exposed, in. ${ }^{2}$ & Dis/min per $\mathrm{ft}^{2}$ \\
\hline White Prime Paint Coat & $\begin{array}{l}55 \\
55\end{array}$ & $\begin{array}{l}518 \\
518\end{array}$ \\
\hline Bare Fir Wood & $\begin{array}{l}55 \\
55\end{array}$ & $\begin{array}{l}1450 \\
1440\end{array}$ \\
\hline White Enamel Paint & $\begin{array}{l}55 \\
55\end{array}$ & $\begin{array}{l}2330 \\
2419\end{array}$ \\
\hline White Latex Paint & $\begin{array}{l}55 \\
55\end{array}$ & $\begin{array}{l}2520 \\
3427\end{array}$ \\
\hline Green Slate & 16 & 4032 \\
\hline Texas Dolomite (White) & 21 & 4766 \\
\hline Sparkle Slate & 20 & 5198 \\
\hline White Artificial Stone & 18 & 5342 \\
\hline Brown Slate & 17.5 & 5659 \\
\hline Black Slate & 16.6 & 6667 \\
\hline Red Weeping Brick & 20 & 6725 \\
\hline Pumice Block & 19 & 6984 \\
\hline Silver Plated Copper & 19 & 5458 \\
\hline
\end{tabular}

surface area; some of the building stones and artifical materials have, of course, a much larger surface area than that of the mere geometric dimensions of the sample. The physical sizes of most of the samples were alike so that air disturbance would be similar in all cases. The large tested area for the wooden pieces was the result of more pieces tested and not of larger samples.

It can be seen that the primed painted wood sorbs the least amount of ${ }^{131} \mathrm{I}$, while a common building material, pumice stone, was highly sorbent. For comparison purposes, data are included from testing silver-plated copper which has an iodine-reactive surface.

About 600 to 700 air samplers were utilized for sampling purposes during Run 2. Figure 95 illustrates the component parts of the iodine field samplers. These samplers contained various 


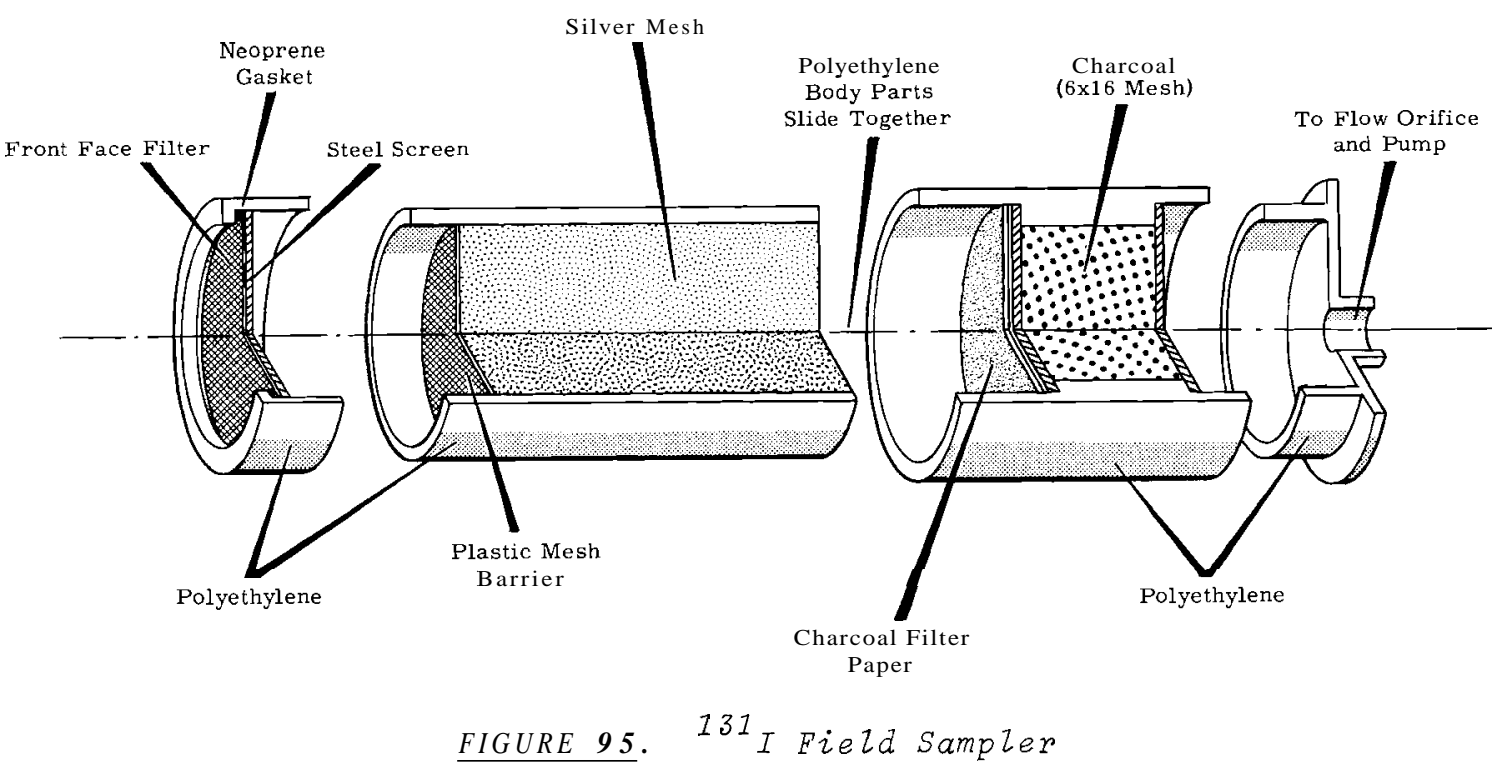

amounts of ${ }^{13}$ I as the result of Run 2 exposure. The overall program for iodine investigation required that a larger quantity of radioiodine be emitted than the $0.4 \mathrm{C}$ i in Run 2; consequently, it was necessary to prepare for a third major field test within a short time. A sufficient number of samples was analyzed from Run 2 to allow a reasonable estimate of ${ }^{131}$ I activity to be made throughout the entire field network. From these data it was found that about 250 samplers had insignificant amounts of activity so they were repositioned into the field sampling network along Arcs 1 and 2. Only their front-face membrane filters were changed. Small amounts of iodine contamination on samplers along the near-in arcs would have a negligible effect on the results of a future test, since high level ${ }^{131}$ I exposure was anticipated at these positions. Over 400 new samplers were assembled and positioned in the field to await Run 3, along with the reused samplers, making a total of about 700 sampling stations.

Instrumentation used to measure the quantity of ${ }^{131}$ I collected by the various parts of the samplers included a variety of gamma-ray spectrometric counting equipment. The front-face filter was placed in a 1 dram vial and counted in a 3 in. NaI(T1) we11type crystal. The silver-plated copper mesh contained within the plastic sleeve was counted intact by inserting it into a housing maintained between two 9 by 4 in. NaI(T1) crystals enclosed in a plastic phosphor backgroundreducing shield, normally used for coincidence work. Measurement of the charcoal filter paper was made in a 5 in. NaI(T1) well crystal housed in a lowlevel anticoincidence arrangement. Activity collected in the charcoal section was measured by removing the charcoal to a jar which was then inserted in a 9 in. NaI(T1) well crystal for gamma assay. All instruments used 
to measure the ${ }^{131}$ I activity were crosscalibrated with each other for the particular geometric arrangement necessary in counting actual field samples.

\section{Iodine Run No. 3}

About $1.8 \mathrm{Ci}$ of ${ }^{131} \mathrm{I}$ were released in a controlled manner to the Hanford meteorological field grid. Atmospheric conditions at the time, September 2, 1965, can be described as moderately stable with respect to lapse rate. The molecular iodine gas was generated by oxidation of the iodide by nitrite from an acid solution. Previous tests using this technique and the field sampling. devices had indicated that a molecular, reactive species was obtained in this manner. Only about $1 \%$ of the iodine activity in these laboratory tests was found to be sorbed onto the front face filter. The remaining iodine species were collected on the first part of the silver mesh section. Essentially no activity was passed to the charcoal component sections.
Seven grass plots were cultivated in an arc at $200 \mathrm{~m}$ from the iodine release point. A large pasture was also cultivated along the $800 \mathrm{~m}$ arc and samples of both plots as well as other vegetation were measured for ${ }^{131}$ I deposition. Table XXXVIII illustrates data taken from grass samples at 200 and $800 \mathrm{~m}$ from the release point.

These data represent results from ${ }^{131}$ I assay of selected grass samples taken at three different times subsequent to the ${ }^{131}$ I release. Only the green upper leaf portions were collected (upper $80 \%$ ). The first samples were taken on the morning of the experiment and one would expect very little depletion of ${ }^{131}$ I from the plant since the deposition. The second series was taken 6 days 1 ater and the thrid series after about 8 additional days. All results were corrected for decay back to the time of emission so any loss in activity must be attributed to causes other than decay.

There are, perhaps, several conclusions one might venture from these

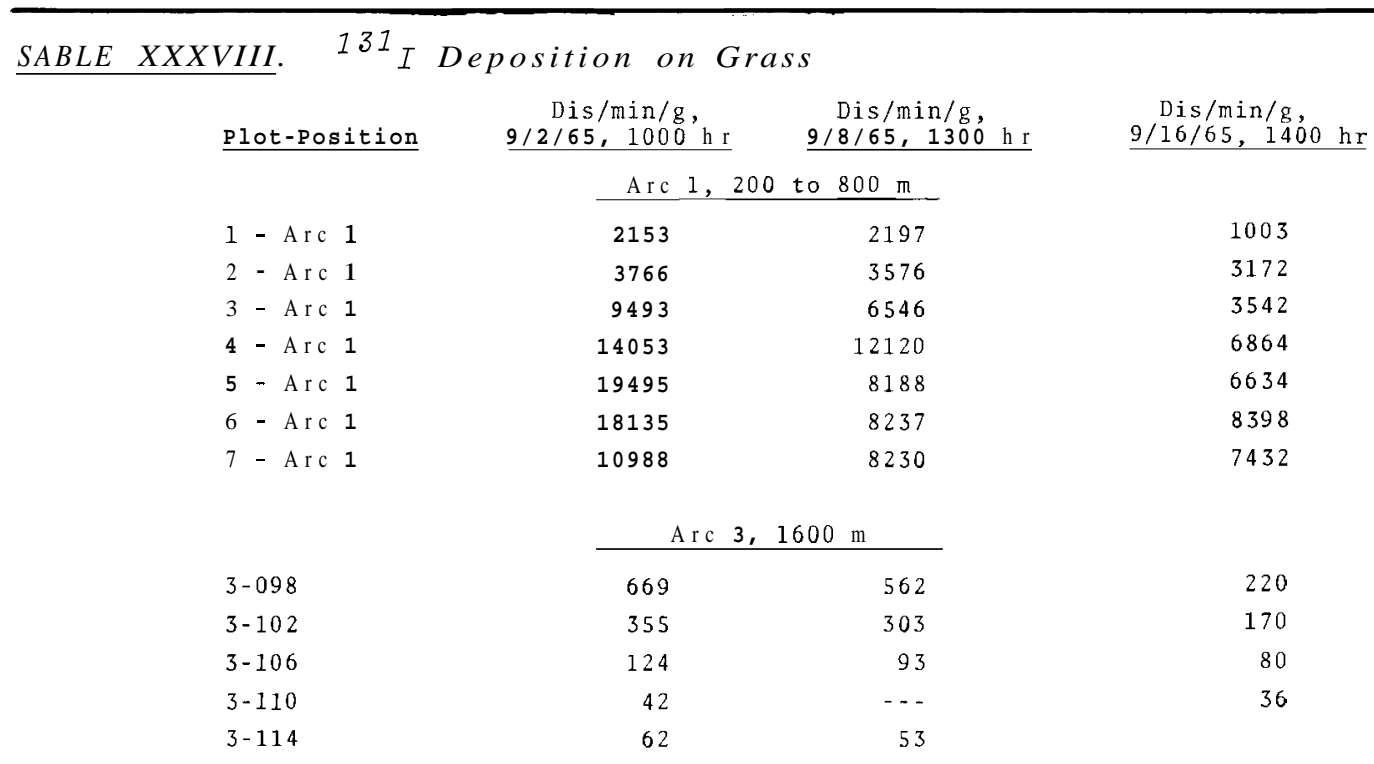


data. There was no reported precipitation between the period $9 / 2$ to $9 / 8$; therefore, one could conclude that any difference in activity between these two sampling periods would be attributed to plant expiration, to sampling variations, or to root takeup or lower level (vertical) concentration. The samples seem to fall into two groups, those in which the activity stayed essentially the same, and any small decreases in specific activity attributable to normal plant growth, and those in which a significant loss of ${ }^{131}$ I occurred. The fact that in some cases the iodine stayed essentially the same, except for radioactive decay, even after 6 days, indicates that absorption into the plant takes place in a very short time subsequent to deposition so that the iodine is not available for weathering. The fact, however, that some samples have a large loss of iodine indicates that at some stage of growth, iodine was available for a release process or that iodine was being held in lower levels of the grass and/or roots.

To follow this line of thought, compare the differences between the samples taken on $9 / 8$ and those taken on 9/16. Again they fall into two groups, those that remained essentially constant and those that lost a considerable fraction of their ${ }^{131}$ I activity. It is also interesting to note that the day before the last samples were taken, a heavy rain fell, 0.5 in. on 9/15/65. The results indicate simply a continuation of the processes which were already occurring with no apparent significant increase in depletion due to the wash. This again points out to a strong attachment of the ${ }^{131}$ I to the grass at least until the grass is ready to release it.

Results from the assay of sagebrush foliage are illustrated in Table XXXIX. Samples were collected immediately following the radio-iodine release and consisted of only the green outer top sections of the bush. Additional samples were taken 6 days later on Arc 3 at $1600 \mathrm{~m}$ to determine possible depletion. Again, a 11 numbers listed were corrected for decay back to iodine release time.

Tables XL and XLI are compilations of the air sampling information taken

TABLE XXXIX. ${ }^{131}$ I Deposition on Vegetation Samples, Dis/min/g

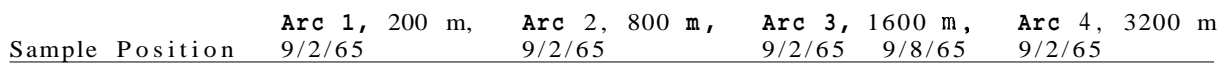

$\begin{array}{rrrrrr}\text { P-094 } & 354 & 25 & 20 & 12 & \\ \text { P-098 } & & 68 & 128 & 69 & 17 \\ \text { P-102 } & 6,680 & 610 & 98 & 72 & 9 \\ \text { P-106 } & 10,270 & 749 & 22 & 14 & 1.5 \\ \text { P-110 } & 6,817 & 209 & 5 & & 2.6 \\ \text { P-114 } & 3,409 & 41 & 8 & & 3.4 \\ \text { P-118 } & 707 & 16 & 7 & & 4.3 \\ \text { P-122 } & 27 & 26 & 10 & & 6.5\end{array}$


a t ground level $(1.5 \mathrm{~m})$ and tower positions during the ${ }^{131}$ I test release No. 3. One can note the very excellent agreement between the average percentage concentration of ${ }^{131} \mathrm{I}$ on each section of the samplers when comparisons are made between ground and tower samples. This was not observed in Run No. 1. (138) If a comparison is made between the results of Runs Nos. 1 and 3 , several factors are found to vary to a considerable extent, and in fact, these differences are useful in a discussion of Run No. 3 itself. From
Arc $1(200 \mathrm{~m})$ to Arc $4(3200 \mathrm{~m})$ in Run No. 3, a change in the percentage of particulate is observed starting at about $3 \%$ on Arc 1 and doubling to about 6\% at Arc 4. Thus only a small amount of the iodine was changed into particulate through interaction with itself or with dust; while during Run No. 1 (data included for comparison purposes on Table XLII, (138) about $10 \%$ of the iodine was already particulate at Arc 1, with over one-third found to be collected on the front-face filter a t Arc 4. This decreased percentage

\begin{tabular}{|c|c|c|c|c|c|c|c|c|c|c|c|c|c|}
\hline \multirow[t]{7}{*}{$\frac{T A B L}{\text { on } T}$} & $L E \quad X L$. & ${ }^{131} I$ & $i n$ & Air Sa & ampler $S$ & ections & s Taken & fron & \multicolumn{2}{|c|}{$n$ Ground L } & Level to & \multicolumn{2}{|c|}{$100 \mathrm{ft}$} \\
\hline & $\begin{array}{l}\text { Sample } \\
\text { azimutl }\end{array}$ & Location, & & $\begin{array}{l}\text { Samplers, } \\
\text { quantity }\end{array}$ & $\begin{array}{l}\text { Front Fi } \\
\text { Particul }\end{array}$ & $\begin{array}{l}\text { ter, } \\
\text { te }\end{array}$ & $\begin{array}{l}\text { Silver M } \\
\text { reactive. }\end{array}$ & esh, & $\begin{array}{l}\text { Charcoal } \\
\text { Paper }\end{array}$ & Filter, & $\begin{array}{l}\text { Charcoal } \\
1.5 \text { in. }\end{array}$ & 1 Mesh, & \\
\hline & & & & & Dis/min & $\%$ & $\underline{\mathrm{Dis} / \mathrm{min}}$ & $\underline{\underline{g}}$ & Dis/min & $\%$ & Dis/min & $\bar{q}$ & \\
\hline & Arc 1 & $068-130^{\circ}$ & & 32 & 69,500 & $3 . \overline{9}$ & $1, \overline{634,000}$ & 91.0 & 78,000 & 4.3 & 13,700 & $0 . \overline{8}$ & \\
\hline & $\operatorname{Arc} 2$ & $082-118^{\circ}$ & & 19 & 7,000 & 4.6 & 137,400 & 90.7 & 5,400 & 3.6 & 1,750 & 1.2 & \\
\hline & $\operatorname{Arc} 3$ & $084-110^{\circ}$ & & 26 & 5,070 & 6.0 & 74,700 & 87.8 & 3,600 & 4.2 & 1,670 & 2.0 & \\
\hline & Arc 4 & $085-106^{\circ}$ & & 18 & 3,750 & 6.7 & 47,100 & 83.7 & 4,560 & 8.1 & 840 & 1.5 & \\
\hline
\end{tabular}

TABLE XLI. ${ }^{131}$ I in Air Sampler Sections Taken $1.5 \mathrm{~m}$ Above Ground, Run 3

\begin{tabular}{|c|c|c|c|c|c|c|c|c|c|}
\hline $\begin{array}{l}\text { Tower Location, } \\
\text { azimuth }\end{array}$ & $\begin{array}{l}\text { Samplers, } \\
\text { quantity }\end{array}$ & $\begin{array}{l}\text { Front Fi } \\
\text { particul }\end{array}$ & & $\begin{array}{l}\text { Silver } \\
\text { reactive }\end{array}$ & & $\begin{array}{l}\text { Charcoal } \\
\text { paper }\end{array}$ & Filter, & $\begin{array}{l}\text { Charcoal } \\
1.5 \mathrm{in} .\end{array}$ & Mesh, \\
\hline & & Dis/min & $\%$ & Dis/min & $\%$ & Dis/min & $\%$ & Dis/min & $\underline{q}$ \\
\hline $1-098$ & 15 & 11,200 & 3.2 & 318,000 & 91 & 18,000 & 5.0 & 2,700 & 0.7 \\
\hline $1-106$ & 16 & 86,000 & 4.3 & $1,790,000$ & 89.7 & 110,900 & 5.5 & 16,400 & 0.8 \\
\hline $1-114$ & 16 & 44,000 & 2.5 & $1,635,000$ & 94.6 & 38,800 & 2.2 & 10,700 & 0.6 \\
\hline $1-122$ & 15 & 2,400 & 3.1 & 70,700 & 91.7 & 2,230 & 2.9 & 1,780 & 2.3 \\
\hline Average, $\%$ & & & $3.5^{\mathrm{a}}$ & & $91.9^{(a)}$ & & $4.1^{(a)}$ & & $0.7^{(a)}$ \\
\hline $2-098$ & 17 & 1,460 & 3.9 & 32,700 & 86.9 & 2,180 & 5.8 & 1,290 & 3.4 \\
\hline $2-106$ & 16 & 19,600 & 4.5 & 398,000 & 90.9 & 17,500 & 4.0 & 2,500 & 0.6 \\
\hline $2-114$ & 17 & 1,250 & 4.0 & 27,700 & 88.1 & 1,070 & 3.4 & 1,420 & 4.5 \\
\hline $2-122$ & 17 & 350 & 2.5 & 11,100 & 80.3 & 960 & 6.9 & 1,420 & 10.2 \\
\hline Average, $\%$ & & & $4.4^{(a}$ & & $90.2^{(a)}$ & & $4.2^{(a)}$ & & $1.3^{(a)}$ \\
\hline $3-098$ & 17 & 6,670 & 5.2 & 110,000 & 86.3 & 9,700 & 7.6 & 1,170 & 0.9 \\
\hline $3-106$ & 18 & 3,800 & 4.4 & 77,000 & 88.6 & 4,900 & 5.7 & 1,200 & 1.4 \\
\hline Average, \% & & & $4.9^{(a}$ & & $87.2^{(a)}$ & & $6.8^{(a)}$ & & $1.1^{(a)}$ \\
\hline $4-098$ & 18 & 5,900 & 6.5 & 76,400 & 84.2 & 7,363 & 8.1 & 1,130 & 1.2 \\
\hline $4-106$ & 17 & 200 & 2.8 & 5,900 & 82.9 & 630 & 8.8 & 390 & 5.5 \\
\hline Average, $\%$ & & & $5.6^{(a}$ & & $84^{(a)}$ & & $8.2^{(a)}$ & & $1.5^{(a)}$ \\
\hline
\end{tabular}

(a) Weighted Average 
TABLE XLII. ${ }^{131}$ I in Air Sampler Sections Taken $1.5 \mathrm{~m}$ Above Ground, Run 1 (138)

\begin{tabular}{|c|c|c|c|c|c|c|}
\hline $\begin{array}{l}\text { Sample } \\
\text { azimuth }\end{array}$ & Location, & $\begin{array}{l}\text { Samplers, } \\
\text { quantity }\end{array}$ & $\begin{array}{l}\text { Filter, } \\
\text { particul }\end{array}$ & & $\underline{\text { Silver, }}$ & reactive \\
\hline & & & $\mathrm{Dis} / \mathrm{min}$ & $\%$ & $\mathrm{Dis} / \mathrm{min}$ & $\%$ \\
\hline Arc 1 & 068-114' & 22 & 18,400 & 9.7 & 139,300 & 73.8 \\
\hline Arc 2 & $072-118^{\circ}$ & 22 & 2,200 & 12 & 11,800 & 65 \\
\hline Arc 3 & $085-110^{\circ}$ & 24 & 1,300 & 22.5 & 3,000 & 51 \\
\hline Arc 4 & $090-106^{\circ}$ & 15 & 900 & 30 & 1,000 & 34 \\
\hline Arc 4 & $\begin{array}{l}071-079^{\circ} \\
109-115^{\circ}\end{array}$ & 14 & 330 & 37 & 430 & 48 \\
\hline
\end{tabular}

\begin{tabular}{|c|c|c|c|}
\hline \multicolumn{2}{|c|}{$\underline{\text { Filter, Charcoal }}$} & \multicolumn{2}{|c|}{ Backup Charcoal } \\
\hline Dis/min & 8 & Dis/min & $\frac{0}{8}$ \\
\hline 15,000 & 8.0 & 16,100 & 8.5 \\
\hline 1,300 & 7.4 & 2,800 & 15.6 \\
\hline 600 & 10.5 & 900 & 16 \\
\hline 200 & 8.0 & 800 & 28 \\
\hline none pre & esent & 140 & 16 \\
\hline
\end{tabular}

existing in the particulate form is believed to be due mainly to the decreased content of atmospheric dust under the more stable atmospheric conditions.

The second section of the iodine collector, containing silver mesh and collecting all reactive species of iodine, held most of the ${ }^{131}$ I from Run No. 3. In Arc 1 samplers, the silver mesh sections contained at least $91 \%$ of the iodine, and this decreased only slowly with distance to Arc $4(84 \%)$ due first to an increase in particulate (almost half of the decrease in reactive iodine from Arc 1 to Arc 4 can be attributed to change to particulate). The remainder of the change in the form of the iodine can be attributed to change to unreactive organic forms collected on the charcoal filter section. A comparison of reactive iodine from Run No. 1 with that from Run No. 3 shows that the greatest differences can probably be attributed to the large amount of particulate iodine in Run No. 1 thus reducing, to a large extent, the amount of reactive iodine available to the silver section. This does not account for all of the difference since it can be seen that in Run No. 1 a great deal of the iodine was converted into unreactive (possibly organic) forms.

There appeared to be a slow conversion of reactive iodine into a form collectable on the charcoal filter (organic unreactive). This is seen as the iodine collection changes from 4 to $8 \%$ with downwind travel. The most remarkable difference that was observed between the two runs was in the amount of rather unreactive iodine collected on the charcoal mesh section. During Run No. 3 only a small portion of the iodine was found in this form, 0.8 to $2 \%$, whereas Run No. 1 contained up to $28 \%$. Obviously a much different chemical process was taking place during Run No. 3. Perhaps the availability of organic molecules in the atmosphere was much reduced; consequently, few iodine molecules were depleted by this process and much more remained in a reactive form to be collected on the silver section.

There appeared to be a rather constant amount of ${ }^{131}$ I collected on the charcoal back-up section away from the main atmospheric stream of iodine. This must be interpreted as a background. Thus when one collects only small amounts of ${ }^{131}$ I in a sampler, the percentage found on the last section (the charcoal 
paper) becomes a much greater percentage of the total found. The samplers used in the present experiments were sufficiently high in ${ }^{13}$ I to avoid misinterpretation due to this factor.

Examples of this can be seen by scanning the analytical data from Arc 1 Tower $1-130^{\circ} ;$ Arc 2 ground $118-160^{\circ}, 2-130^{\circ}$

Tower; Arc 3 ground $112-160^{\circ}, 3-114^{\circ}$

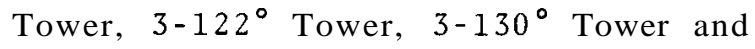
other similar positions on Arc 4. These data were not included since they represent little more than background activity levels.

\section{ORGANIC ${ }^{131}$ I COMPOUNDS RELEASED FROM A NUCLEAR FUEL CHEMICAL PROCESSING}

PLANT*-W. A. H a II e r

\begin{abstract}
Organic ${ }^{131}$ I compounds which are present in the effluent from a nuclear fuel processing plant were fractionated by gas chromatography and measured by gamma-ray spectroscopy. The analyses of the effluent organic ${ }^{131}$ I materials showed a complex mixture of compounds with boiling ranges of 40 through $255^{\circ} \mathrm{C}$; the lower boiling compounds were identified as methyl, ethyl, n-propyl and $n$-butyl iodides. The methyl and $n$-butyl iodide and the materials in the boiling range pentyl through heptyl iodide contained approximately $70 \%$ of the observed alkyl iodides.
\end{abstract}

* To be published in Analytical Chemistry

On September 29, 1965, at 0510 a purposely defected, partially molten fuel rod in the rupture loop test facility of the Plutonium Recycle Test Reactor (PRTR) failed in an unexpected manner. The rupture, which resulted in a loss of about $39 \%$ of the $\mathrm{UO}_{2}-\mathrm{PuO}_{2}$ fuel from the rod, was accompanied by the formation of a hole of approximately $1 / 2$ in. diam in the surrounding process tube. The subsequent flashing of the highly contaminated superheated water released a fission product aerosol to the containment vessel atmosphere. This release resulted in about half of the noble gas content reaching the containment vessel atmosphere. About $1 \%$ of the radioiodine and a somewhat smaller fraction of the solid fission products entered the containment vessel atmosphere. A subsequent radiochemical study provided detailed information on the behavior of some 15 fission products and of plutonium in their movement, following the rod failure, through the various liquid and gaseous systems of the reactor and its containment vessel. The ratios of the fission products (other than the noble gases) which were released to the containment vessel atmosphere were not far different from those in the fuel rod showing that relatively little fractionation occurred in the process of dissolution and aerosol formation. 
Only a small fraction of the ${ }^{131} \mathrm{I}$ and other fission products which were released to the containment vessel atmosphere were deposited on the containment vessel surfaces. About $99 \%$ of the fission product aerosol was removed in the condensate from air coolers which processed about $6 \%$ of the containment vessel's atmosphere per minute. The ratios of the solid fis sion products which were deposited on surfaces in the containment vessel were quite similar to those in the fuel rod. ${ }^{141} \mathrm{Ce}$ and ${ }^{95} \mathrm{Zr}-{ }^{95} \mathrm{Nb}$ were often exceptions, running several-fold lower on the surfaces. The ratio of radioiodine to other fission products was about 10 times higher on the walls and ceilings than on floor surfaces on the first 2 days following the incident, but after 6 days the ratios were comparable. The deposition of the radioactive aerosol per unit of surface area was about 4 to 5 times greater on the floors than on the wall or ceiling surfaces. The observed deposition per unit area on metal surfaces was much higher than on painted surfaces. The behavior of the radionuclides in this system was similar to what might be expected in the meltdown of any water-cooled reactor and the information gained from the study should be applicable to the design of safeguards for such events.

\title{
A SIMPLIFIED METHOD FOR THE DETERMINATION OF TRACE ELEMENTS IN GEOLOGIC
} SAMPLES BY ACTIVATION ANALYSISX-W. B. Silker

\begin{abstract}
A combination of neutron activation, simple chemical treatment and gamma-ray spectrometry was applied $i n$ the determination of trace elements in basalt samples. The simplicity of the procedure permitted completion of the separations $i n 2$ to $3 \mathrm{hr}$ and provided measurement of the concentrations of 15 elements including $?$ rare earths. Accurately weighed $10 \mathrm{mg}$ aliquots of powdered basalt samples were sealed in vitreous silica ampules and, together with an aliquot of a basalt whose trace element concentrations had been accurately determined, were irradiated for 5 min at a neutron flux of at least $5 \times 10^{13} \mathrm{n} / \mathrm{cm}^{2}-\mathrm{sec}^{1}$. I $t$ was found early in the work that the composition of the basalt was such that the neutron-induced radioisotopes of sodium and manganese effectively masked the other radioactive species present. The following technique was therefore developed to separate other radioisotopes of interest from these two elements prior to analysis by gamma-ray spectrometry. After sample dissolution by treatment with HF, HNO, and HCZO, the residue was dissolved in 10M HCl, and most metalZic elementswere retained (as anionic complexes) on a column of anion exchange resin through which the solution was passed. The rare earth elements were then isolated by coprecipitation with lanthanum fluoride from the column effluent, which also contained manganese, sodium, alkaline earths, and such anionic constituents as sulfate and phosphate. Measurements of the various fractions by gamma-ray spectrometry permitted determination of the concentrations of the following elements in the sample: sodium,
\end{abstract}


manganese, copper, gallium, zinc, iron, cobalt, uranium, lanthanum, cerium, samarium, europium, dysprosium, ytterbium, and lutetium. The elemental concentrations were determined within a deviation of 5 to $20 \%$ at the $68 \%$ confidence limit. This precision will permit fair estimation of the trace element concentrations in a variety of samples and, because of the relatively simple chemistry involved, would be quite adequate for survey type applications.

* Submitted for publication in Geochemica and Cosmochemica Acta

IDENTIFICATION OF INDIVIDUAL BASALT FLOWS BY ACTIVATION ANALYSIS - W. B. Silker

The geology of basalt flows in southeastern Washington, particularly those of the Pasco Basin, which includes the Hanford reservation, has been the subject of many investigations. The problems encountered in this work as reviewd by Brown and Brown (142) are centered primarily around positive identification of specific basalt flows and the recognition of these flows in other nearby areas. Most of the basalt formations were named and typed from the localities in which they were first observed. Subsequent identification of these flows at other locations is based on physical and microscopic properties and recognition of the sedimentary deposits bounding them. In many instances, lack of suitable contacting sediments, or the occurrence of erosion following deposition complicated the identification of a particular flow with one that had been previously characterized. This has, in some instances, resulted in confusion in the nomenclature of the various flows and has indicated a distinct need for a method of identification.

Knowledge of the origin and boundaries of each individual flow is also of interest in the definition of the geologic history of the area. $(142,143)$

Previous work by the author revealed significant differences in the concentration of certain trace elements in Pomona and Elephant Mountain basalt, two adjacent flows. The trace element concentrations appeared to be constant within each flow with little, if any, vertical fractionation. This present paper describes an extension of those studies to determine the feasibility of differentiating additional basalt flows by their characteristic trace element concentrations.

A series of samples was obtained from well No. 13/24-2851 which had been core drilled to the artesian aquifer and which included most of the flows of the Yakima Formation. The specimens were taken from 10 to $20 \mathrm{ft}$ above the base of the particular flow and were identified by R. E. Brown from the drilling record and microscopic examination. The samples were analyzed in duplicate by the neutron activation method detailed in the preceding paper. The concentrations of 15 elements are presented in Table XLIII. 
TABLE XLIII. Elemental Abundance in Individual Basalt FZows, ppm

\begin{tabular}{|c|c|c|c|c|c|c|c|}
\hline & $\begin{array}{l}\text { Elephant } \\
\text { Mountain }\end{array}$ & Pomona (a) & $\begin{array}{l}\text { Priest } \\
\text { Rapids No. } 3 \\
\end{array}$ & $\begin{array}{l}\text { Priest } \\
\text { Rapids No. } 2 \\
\end{array}$ & $\begin{array}{l}\text { Priest } \\
\text { Rapids No. } 1 \\
\end{array}$ & $\underline{\text { Roza }}$ & $\begin{array}{l}\text { Sand } \\
\text { Hollow }\end{array}$ \\
\hline No. of Analyses & 3 & 7 & 2 & 2 & 2 & 2 & 2 \\
\hline Depth in Well, f t & & & 60 & 140 & 250 & 360 & 570 \\
\hline Sodium & 60,500 & 56,800 & 73,000 & 58,800 & 72,600 & 76,800 & 67,000 \\
\hline Manganese & 1,440 & 1,120 & 1,190 & 1,210 & 1,240 & 1,410 & 1,320 \\
\hline Copper & 22.4 & 37.5 & 7.81 & 37.0 & 44.6 & 9.37 & 28.3 \\
\hline Gallium & 22.5 & 19.1 & 20.9 & 19.8 & 21.8 & 24.1 & 20.8 \\
\hline Zinc & 291 & 165 & 691 & 752 & 351 & 362 & 222 \\
\hline Iron & 110,000 & 78,700 & 82,600 & 84,000 & 98,100 & 91,500 & 96,700 \\
\hline Cobalt & 3.34 & 3.88 & 2.85 & 3.74 & 2.50 & 3.42 & 3.38 \\
\hline Uranium & 2.56 & 1.25 & 2.82 & 1.23 & 2.30 & 3.25 & 3.53 \\
\hline Europium & 2.41 & 1.53 & 4.10 & 1.53 & 3.54 & 3.57 & 3.08 \\
\hline Dysprosium & 10.3 & 6.34 & 9.35 & 5.56 & 6.88 & 8.17 & 10.3 \\
\hline Lutetium & 1.26 & 0.52 & 1.24 & 0.48 & 1.05 & 1.09 & 1.05 \\
\hline Lanthanum & 36.9 & 19.6 & 49.7 & 18.8 & 44.9 & 43.0 & 35.4 \\
\hline Cerium & 20.5 & 13.4 & 57.9 & 24.7 & 44.4 & 38.7 & 29.3 \\
\hline Samarium & 8.17 & 4.93 & 8.64 & 4.71 & 9.07 & 7.30 & 7.38 \\
\hline Ytterbium & 4.39 & 2.68 & 4.24 & 2.64 & 5.11 & 3.29 & 3.80 \\
\hline
\end{tabular}

The precision of replicates was within $10 \%$ for most of the determinations but was quite poor in the cases of zinc, cerium and ytterbium, so suitable reservations should be placed on these values in any evaluation.

It becomes obvious from these data that differentiation between adjacent flows by comparison of their respective trace element concentrations is entirely possible. Certain similarities exist, for example the Sand Hollow and Roza flows, but at the same time notable differences are seen in the respective concentrations of sodium, copper and lanthanum. The concentrations of all measured elements in samples of the Pomona and Priest Rapids No. 2 flows were identical, within experimental error. It would thus be impossible to distinguish between these two flows by analysis of individual samples. If, however, samples of either the overlying or underlying flows were concurrently analyzed, positive identification could then be made. The identity of the Pomona and Priest Rapids No. 2 flows strongly suggests that both were of the same origin. It is interesting to note the increased iron concentration from the Pomona through the Sand Hollow flows, a 11 of which are members of the Yakima Formation. The distributions of the rare earth elements in these basalt samples, normalized to lutetium, were compared with those observed in chondritic meteorites. (144) Lanthanum enrichment was found in all of the current samples. Chondritic rare earth distributions were observed in the samples of basalt from the Elephant Mountain, Priest Rapids No. 3, Roza, and Sand Hollow flows. This represents an undisturbed 
rare earth distribution and suggests that these flows originated from material lying deep within the mantle. The three other flows exhibited a distribution typical of other basalts, with increased enrichment of rare earth elements of decreasing atomic radius.
The degree of enrichment was considerably less than that reported for Kilauea-Iki lava. (144) It is suggested that the degree of rare earth enrichment might provide an index of the depth within the earth's mantle from which the magma was generated.

\section{TRACE ELEMENT IMPURITIES IN VITREOUS SILICA TUBING-D. E. Robertson}

One of the major problems in measuring trace elements by ultra-sensitive activation analysis is the risk of contaminating the sample during handing or pretreatment. A frequent source of contamination is that of the trace element impurities present in the container used for encapsulating the sample for neutron irradiation. This laboratory has extensively used vitreous silica ampules as sample containers, since organic containers such as polyethylene, Teflon, etc., are decomposed during long irradiations by the heat and radiation in the reactor. With the sensitivities afforded by available high neutron fluxes and the application of multidimensional gammaray spectrometry, it has become evident that standard grade vitreous si lic a ampules contained sufficient impurities to make them unsuitable for ultra-sensitive measurements of some trace elements. This was quite apparent during the measurement of certain trace elements in sea water and in biological materials where concentrations lower than 1 ppb are routinely measured using multidiniensional gamma-ray spectrometry after neutron irradiation.
To determine the trace element contributions from various containers, 6 proprietary silica tubing samples were neutron-activated and their trace element concentrations measured, with the ultimate aim of selecting one (or more) for use in ultra-sensitive activation analyses Samples of the tubing were irradiated for 2 hr (to measure short half-life radionuclides) and for 5 to 9 days (to measure long halflife radionuclides) in a thermal neutron flux of over 1 x 1013 neutrons $/ \mathrm{cm}^{2}-\mathrm{sec}$. A cobalt standard was used as a neutron flux monitor for each irradiation. After the 2 hr irradiation the silica samples were allowed to decay for about $24 \mathrm{hr}$ to reduce the Bremsitrahlung interference from ${ }^{31} \mathrm{Si}(2.6 \mathrm{hr})$. Then the samples were gamma-counted for 10 to 100 min using a detector system consisting of two $6 \times 6$ in . $\mathrm{NaI}$ (T1) crystals in coincidence surrounded by plastic phosphor anticoincidence shielding and coupled to a Packard multiparameter analyzer. The following radionuclides were easily measured without performing a chemical separation: ${ }^{24} \mathrm{Na},{ }^{64} \mathrm{Cu}$, 
${ }^{140} \mathrm{La},{ }^{134} \mathrm{Cs}$, and ${ }^{239} \mathrm{~Np}$. The 5 to 9 d irradiated samples were allowed to decay for several weeks to reduce the interference from ${ }^{24} \mathrm{Na}$ and other short half-life radionuclides. Then the samples were gamma-counted for 1000 min using the same counting equipment.

Most of the following radionuclides were readily measured without performing a chemical separation: ${ }^{46} \mathrm{Sc},{ }^{51} \mathrm{Cr}$, ${ }^{59} \mathrm{Fe},{ }^{60} \mathrm{Co},{ }^{65} \mathrm{Zn},{ }^{110 \mathrm{~m}} \mathrm{Ag},{ }^{124} \mathrm{Sb},{ }^{134} \mathrm{Cs}$,

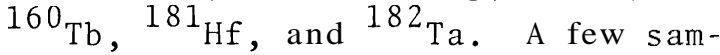

ples contained such high concentrations of certain radionuclides that their Compton interferences made the detection of other trace radionuclides difficult.

The concentration of the parent element of each induced radionuclide in the samples was then calculated. A summary of the data is presented in Table XLIV.

As Table XLIV indicates, the "Spectrosil" and "Suprasil" are in most cases the purer materials. Except for iron, the "Spectrosil" is extremely pure. The "Suprasil" is also very pure, except for relatively higher concentrations of scandium and cobalt. Brand "C" would be unsuitable for ultrasensitive activation analyses because of the extremely large amounts of ${ }^{134} \mathrm{Cs}$ and ${ }^{124} \mathrm{Sb}$ produced from the high cesium and antimony impurities. Brands "D," "E," and "F" also appear to be unsuitable for ultrasensitive work due to the relativelv large amounts of ${ }^{124} \mathrm{Sb}, 140 \mathrm{La},{ }^{160} \mathrm{~Tb}, 181_{\mathrm{Hf}}$. ${ }^{239} \mathrm{~Np}$ and ${ }^{140} \mathrm{Ba}-{ }^{140} \mathrm{La}$ (and other fission products of uranium) produced from their parent impurities.

To estimate the extent that samples would be contaminated from the ampules a simulated activation analysis experiment was conducted using only $0.10 \mathrm{ml}$ of $1.5 \mathrm{~N} \mathrm{HNO}_{3}$ (prepared from redistilled $\mathrm{HNO}_{3}$ and doubly distilled water) as the "sample." Also, empty, sealed ampules were irradiated. "Spectrosil" and "Suprasil" ampules were used, since they appeared best suited for ultrasensitive work. After a 5 d irradiation in a neu

TABLE XLIV. Trace Element Impurities in Six Sample of Proprietary Vitreous Silica Tubing

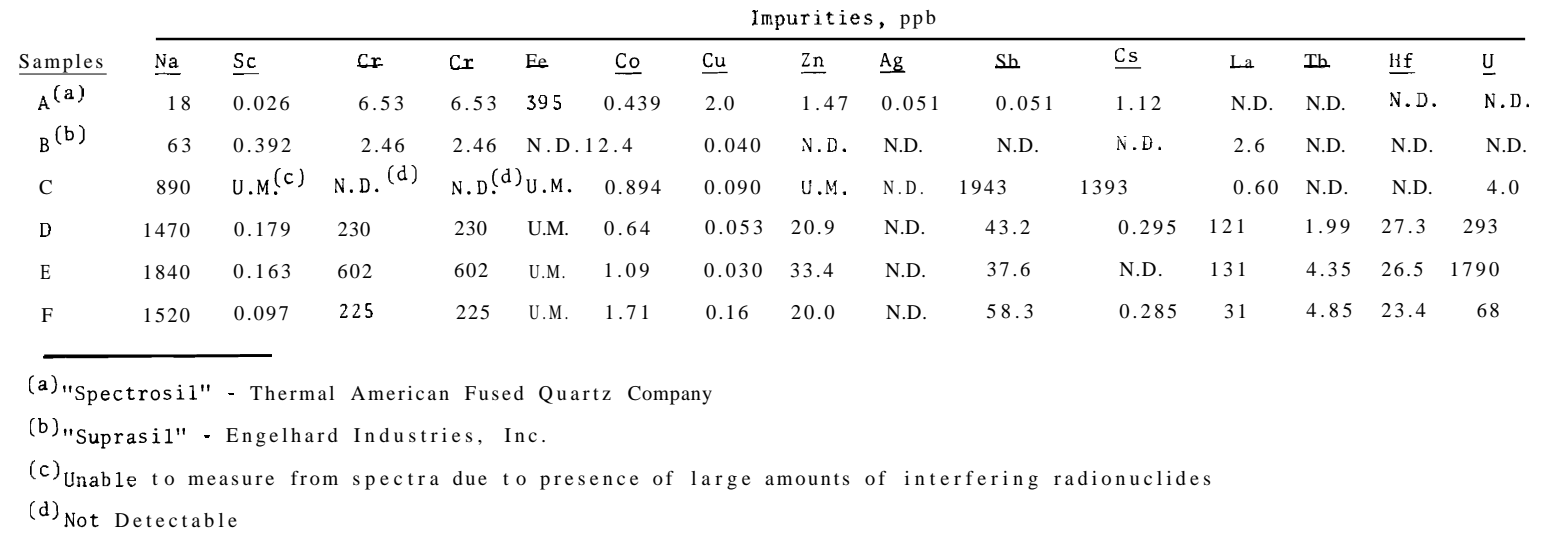


tron flux of over $1 \times 10^{13}$ neutron/ $\mathrm{cm}^{2}-$ sec and a 2 week decay period, the ampules were opened and the $\mathrm{HNO}_{3}$ transferred to a counting dish. The ampules which had contained the $\mathrm{HNO}_{3}$ were then rinsed with $1.0 \mathrm{ml}$ of hot, concentrated $\mathrm{WNO}_{3}$ which was also plated onto a counting dish. The empty ampules were opened and rinsed first with $1.0 \mathrm{~m} 1$ of distilled water which was plated and then with 1.0 $\mathrm{ml}$ of hot, concentrated $\mathrm{HNO}_{3}$ which was also plated. Thus, four alternate meth- ods of postirradiation treatment of the sample were evaluated. The 1. $\underline{5} \mathrm{HNO}_{3}$ and all of the rinse solutions were then counted for 1000 min using the Packard multidimensional analyzer to determine the extent to which the trace element impurity radionuclides were leached from the surface during the treatment. Table XLV is a summary of the data obtained.

It is apparent that the majority of the radionuclide impurities in the $1.5 \underline{N} \mathrm{HNO}_{3}$ used as a "sample" originated from trace element impurities in the $1.5 \mathrm{~N} \mathrm{HNO}_{3}$ itself, since many of their parents are not present

TABLE XLV. Contamination in Activation Analyses Originating from Trace Element Impurities in Vitreous Silica Ampule Sample Containers

\begin{tabular}{|c|c|c|c|c|}
\hline \multirow{2}{*}{$\begin{array}{l}\text { Radionuclide } \\
\text { Contaminant }\end{array}$} & \multicolumn{2}{|c|}{ "Spectrosil" Ampule } & \multicolumn{2}{|c|}{ "Suprasil" Ampule } \\
\hline & $1.55 \mathrm{HNO}_{3}$ "Sample" & Conr. $\mathrm{HNO}_{3}$ Rinse & $1.5 \mathrm{~N} \mathrm{HNO}_{3}$ "Sample" & Conc. $\mathrm{HNO}_{3}$ Rinse \\
\hline${ }^{46} \mathrm{Sc}$ & 57.6 & 0.99 & 31.0 & 3.0 \\
\hline${ }^{51} \mathrm{Cr}$ & 2748 & 15.2 & 1080 & U.M. (a) \\
\hline${ }^{59} \mathrm{Fe}$ & 94.1 & N.D. ${ }^{(b)}$ & 75.3 & U.M. \\
\hline${ }^{60} \mathrm{Co}$ & 59.9 & 1.8 & 30.7 & 1.8 \\
\hline $65_{z_{n}}$ & 633.3 & 0.91 & 351.0 & N.D. \\
\hline $110 \mathrm{~m} A g$ & 83.0 & N.D. & 10.0 & 22.4 \\
\hline $124_{\mathrm{Sb}}$ & 89.3 & N.D. & 48.1 & N.D. \\
\hline $140 \mathrm{Ba}-140 \mathrm{La}$ & 113.0 & N.D. & N.D. & N.D. \\
\hline${ }^{181_{\mathrm{Hf}}}$ & 83.1 & N.D. & 11.6 & U.M. \\
\hline${ }^{239} \mathrm{~Np}$ & 2683 & N.D. & 510 & U.M. \\
\hline $182 \mathrm{Ta}$ & N.D. & N.D. & N.D. & Large amount \\
\hline $134 \mathrm{Cs}$ & N.D. & N.D. & N.D. & N.D. \\
\hline
\end{tabular}

\begin{tabular}{|c|c|c|c|c|c|}
\hline & & $\begin{array}{l}\text { Empty Ampule Irra } \\
\text { Total Dis/min in }\end{array}$ & $\begin{array}{l}\text { tion } \\
\text { ses }\end{array}$ & & \\
\hline & Dist. Water Rinse & Conc. $\mathrm{HNO}_{3}$ Rinse & Dist. & Water Rinse & Conc. $\mathrm{HNO}_{3}$ Rinse \\
\hline${ }^{46} \mathrm{Sc}$ & 1.33 & 3.4 & & 18.4 & 43.0 \\
\hline${ }^{51} \mathrm{Cr}$ & 70.1 & 59 & & 138 & 111 \\
\hline${ }^{59} \mathrm{Fe}$ & N.D. & 6.9 & & 36 & N.D. \\
\hline${ }^{60} \mathrm{Co}$ & 18.3 & 6.4 & & 17.09 & 39.3 \\
\hline${ }^{65} \mathrm{zn}$ & 101 & N.D. & & 126 & N.D. \\
\hline $110 \mathrm{~m}_{\mathrm{Ag}}$ & 8.0 & 7.9 & & 7.9 & 5.3 \\
\hline${ }^{124} \mathrm{Sb}$ & N.D. & 4.6 & & 4.0 & 2.3 \\
\hline${ }^{134} \mathrm{Cs}$ & N.D. & N.D. & & N.D. & N.D. \\
\hline
\end{tabular}

(a) Unable to measure from spectra due to large amounts of interfering ${ }^{182} \mathrm{Ta}$

(b) Not Detectable 
as an impurity in the "Spectrosil" and "Suprasil" materials. However, some contribution is due to radionuclides that were leached from the ampules by the $1.5 \mathrm{~N} \mathrm{HNO}_{3}$. A better indication of which radionuclides are leached from the ampules is seen in the rinses from the empty irradiated ampules where outside contamination was minimized. Although "Spectrosil" contains a relatively high concentration of iron, very little ${ }^{59} \mathrm{Fe}$ is leached from the silica.

A 11 other leached radionuclides, except for ${ }^{65} \mathrm{Zn}$ and ${ }^{51} \mathrm{Cr}$, are very low in concentration. "Suprasil" has relatively higher concentrations of scandium and cobalt than "Spectrosil" and the effect of this can be seen in the higher concentrations of ${ }^{46}$ Sc and ${ }^{60}$ Co in the "Suprasil" rinses. Although these concentrations are very low they are well above the detection limits of the multidimensional analyzer and would contribute some contamination to a sample. However, here too, some slight outside contamination is suspected for some elements which possibly originated during the precleaning process before irradiation, which consisted of boiling in hot, concentrated redistilled $\mathrm{HNO}_{3}$ and rinsing with doubly distilled water.

A high concentration of ${ }^{182} \mathrm{Ta}$ appeared in the concentrated $\mathrm{HNO}_{3}$ rinse of the "Suprasil" ampule which contained the $1.5 \mathrm{~N} \mathrm{HNO}_{3}$. It appears that this ampule had an inclusion of some tantalum-containing material which was leached by the $1.5 \mathrm{~N} \mathrm{HNO}_{3}$ or was present in the $1.5 \mathrm{~N} \mathrm{HNO}_{3}$ itself. Another problem in using silica ampules as sample containers for ac- tivation analyses is that when they are opened, small, often unseen, chips of neutron-irradiated $\mathrm{SiO}_{2}$ sometimes fall into the sample, resulting in further contamination.

This, perhaps, explains the presence of certain radionuclides in the water rinse of the empty irradiated ampules where very small chips could have been transferred with the water.

In performing ultrasensitive activation analyses it is essential that one knows or can estimate the sources of error in a measurement. The error originating from contamination can be greatly reduced by selecting very pure tubing, such as "Spectrosil" or "Suprasil," to prepare the irradiation ampules. During the handling and shipping of the tubing, it is exposed to dust particles and other contaminants. Therefore, the ampules should be thoroughly precleaned by boiling in concentrated redistilled $\mathrm{HNO}_{3}$ and rinsing in doubly distilled water or by some other equivalent process. There should be no or very little pretreatment of the sample, since this increases the probability for contamination to occur. When it is possible and convenient, a dry sample with no rinsing of the ampule after irradiation is preferred. When rinsing of the ampule is required to remove a 11 of the sample, as $1 \mathrm{itt}$ le rinsing as possible should be used to avoid leaching out radionuclide impurities in the tubing. By following these precautions, the contamination in activation analyses due to impurities in the sample container can be greatly reduced. 
PRELIMINARY EVALUATION OF AN EXISTING MULTIDIMENSIONAL GAMMA-RAY SPECTROMETER FOR WHOLE BODY COUNTING-D. E. Robertson and R. W. Perkins

A preliminary evaluation of a multidimensional gamma-ray spectrometer as a whole body counter was conducted. The detection sensitivity for it s $93 / 8$ in. diam by 4 in. thick crystals was compared with that of a single NaI(T1) crystal of this size with and without anticoincidence shielding and light piping. The detection sensitivities for coincidence counting of ${ }^{60} \mathrm{Co}_{\mathrm{O}}$ and of $226_{\mathrm{Ra}}$ daughters by the coincidence system and single crystal were comparable. Although the multidimensional system had a background several orders of magnitude lower, its coincidence counting efficiency was also much lower at the geometries required for whole-body counting. The multidimensional system of this size would be most useful in measuring complex mixtures of radionuclides in the body since Compton interferences are extremely small, and the detection sensitivities would remain fairly constant even though many radionuclides were present. The use of larger crystals would improve the sensitivity for coincidence counting relative to single crystal counting.

Whole-body counting is useful in studying the uptake, distribution, retention and excretion of various radionuclides by the body. These radionuclides may result from fallout of nuclear weapons, from activation and fission product releases from nuclear installations, or other sources. They may enter the body either by accidental admittance into persons who work with radioactive materials or via the food chain through plants and animals which are consumed by humans. Because the uptake resulting from these radionuclides is, in most cases, extremely small compared with the maximum permissible body burden, their detection and measurement (with the possible exception of ${ }^{137} \mathrm{Cs}$ ) may be difficult or impossible with present technology. Very sensitive detector systems are required to measure the low concentrations of radionuclides in the body, which are often present as minor constituents of a complex mixture of radionuclides.

Multidimensional gamma-ray spectrometry has proven to be an extremely useful tool in analyzing complex mixtures of radionuclides whose constit- uents vary in concentration by orders of magnitude. $(98,102,145,146)$ detector systems have provided improved selectivity over conventional gammaray spectrometers for most applications. This present work was conducted to evaluate the use of a multidimensional gamma-ray spectrometer at the relatively poor geometry used in whole-body counting.

\section{Design of Detector System}

Several multidimensional gamma-ray spectrometry detector systems, utilizing various crystal sizes and anticoincidence shielding, have previously been described. $(98,102,145)$ The system selected for evaluation as a whole body counter consists of two $93 / 8$ in. diam by 4 in. thick NaI(T1) crystals operated in coincidence and surrounded by 30 in. diam plastic phosphor anticoincidence shielding (see Figure 96). The upper $\mathrm{NaI}(\mathrm{T} 1$ ) crystal is fitted with a $93 / 8$ in. diam by 8 in. thick Lucite light pipe to reduce background radiation originating in the phototube. This crystal is clad in 19 mil stainless steel 


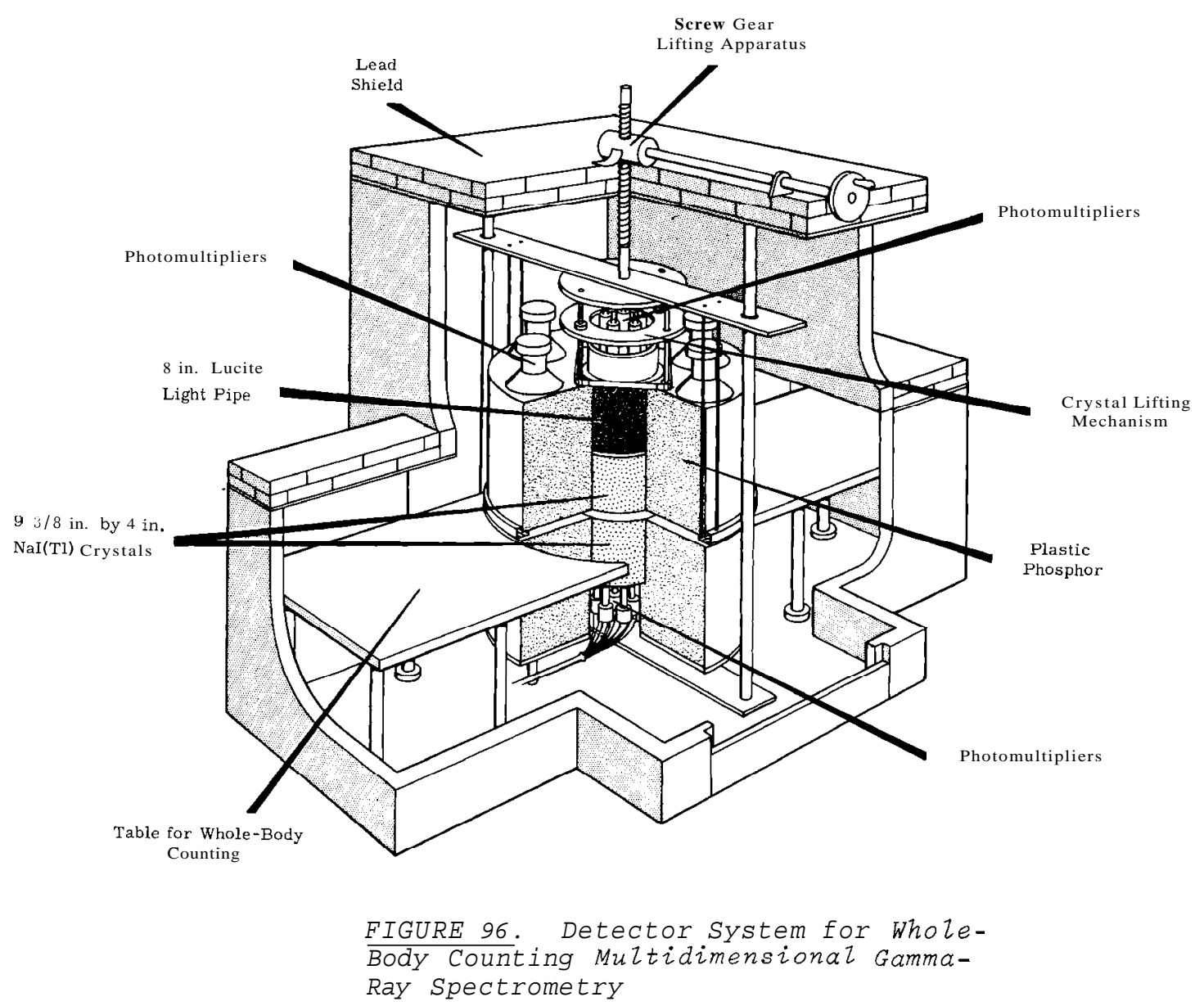

and is viewed by four 3 in. multiplier phototubes. The lower NaI(T1) crystal is identical with the upper crystal except that it has no light pipe. The resolution of the upper crystal with the $1 \mathrm{ight}$ pipe was $9.4 \%$ and the lower crystal had a resolution of $8.8 \%$ as measured at the $662 \mathrm{keV}{ }^{137} \mathrm{Cs}$ gamma-ray energy. Each NaI(T1) crystal was surrounded by a $30 \mathrm{in}$. diam by $15 \mathrm{in}$.

thick plastic phosphor anticoincidence shield. Each phosphor was viewed by six 5 in. EMI multiplier phototubes (No. 9530B). The upper phosphorcrystal assembly could be raised and lowered by a lifting jack. The upper NaI(T1) crystal also could be inde- pendently raised and lowered by a smaller lifting jack mounted on the top plate of the upper phosphor (see Figure 96). The lead cave was modified to accomodate an iron table extending on both sides of the lower phosphor-crystal assembly to allow a person to lie between the upper and lower detector assemblies during a whole body count. The amplified signals from the two $\mathrm{NaI}(\mathrm{T} 1)$ crystals were fed into the dual analog to digital converter of a Packard 4096 channel analyzer for energy and coincidence analysis and from there to the computer memory of the analyzer. When a single gamma-ray interacts 
with either one of the crystals, the event is recorded in the corresponding $X$ or $Y$ axis of the memory as in normal gamma-ray spectrometry. When two (or more) photons simultaneously interact, each in a separate crystal, the event is stored in the energy-energy plane of the memory at a point uniquely characteristic of the two (or more) photon energies. When the two detectors are very close together, the detector system approaches a total absorption gamma-ray spectrometer, since most of the photons which are not totally absorbed in the $\mathrm{NaI}(\mathrm{T} 1)$ crystals are cancelled by the anticoincidence shielding.

Background Measurements and Compton Reductions

Background measurements were made for each $93 / 8$ in. diam by 4 in. thick NaI(T1) crystal with and without anticoincidence to illustrate the benefit of background reduction by anticoincidence shielding and light piping (see Figure 97). With anticoincidence the background is reduced to about

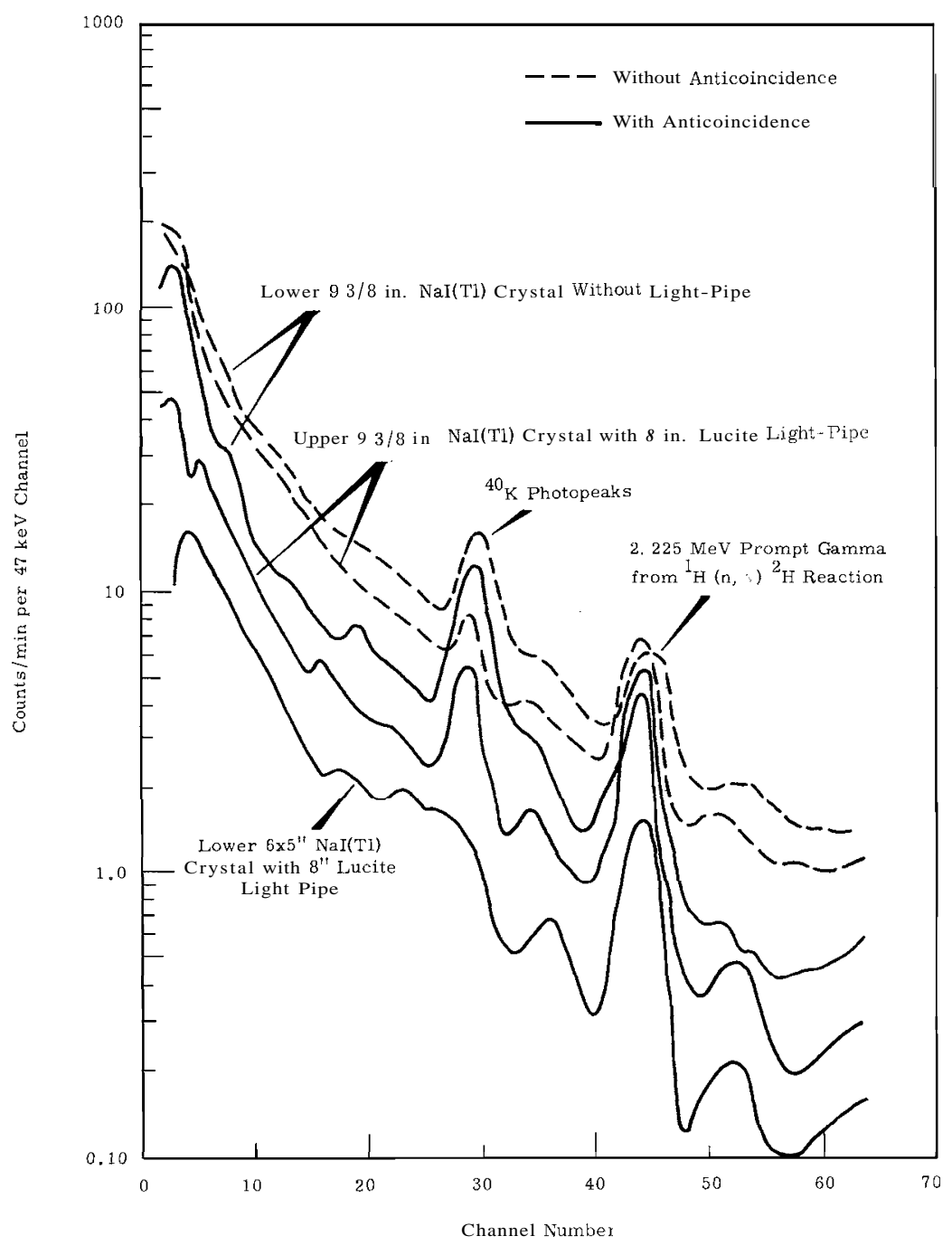

FIGURE 97. Comparison of Single Crystal Backgrounds with and Without Light Pipe and Anticoincidence Shielding 
one-third over most of the energy range from 0.1 to $3 \mathrm{MeV}$. The light pipe on the upper $\mathrm{NaI}(\mathrm{T} 1)$ crystal further reduces the remaining background to onehalf over most of the same energy range by reducing the contribution from ${ }^{40} \mathrm{~K}$ and other radionuclides in the phototubes. The efficiency of the anticoincidence shielding is somewhat limited by the relatively thick stainless steel cladding on the crystals. Some Compton photons escaping the $\mathrm{NaI}$ (T1) detectors interact with their cladding and thus are not able to interact with the phosphor and cancel the event. The large peak in the backgrounds at $2.225 \mathrm{MeV}$ results from cosmic-ray interactions with the lead shielding and plastic phosphors.

Cosmic-ray showers produce neutrons in the lead shielding which are thermalized in the plastic phosphors and captured by hydrogen atoms which deexcite by the emission of a $2.225 \mathrm{MeV}$ prompt gamma-ray. A fraction of these gamma-rays lose their full energy to the $\mathrm{NaI}(\mathrm{T} 1)$ crystals, resulting in the large peaks in the background curves. Studies are presently being made to reduce this interference. The background of a 6 in. diam by 5 in. thick NaI(T1) crystal with an 8 in. thick Lucite light pipe and which was also surrounded by a $30 \mathrm{in}$. diam plastic phosphor anticoincidence shield is compared with the $93 / 8$ in. crystals in Figure 97. This crystal exhibits essentially no ${ }^{40} \mathrm{~K}$ interference and a lower background because of its smaller $\mathrm{s}$ i z e.

Background measurements of the energyenergy plane of the $93 / 8$ in. crystal system were made with the detectors $1 / 8$ in. and $10 \mathrm{in}$. appart and also at 10 in. apart with a "torso water bath" sandwiched between the detectors to simulate the thorax of a person. These measurements are for an energy diagonal extending from 0 to $3 \mathrm{MeV}$ (see Figure 98). The "torso water bath" consisted of an $8 \mathrm{in}$. deep by $111 / 2$ in. wide, water-filled polyethylene container in the shape of a cylindroid. The backgrounds were taken with and without anticoincidence. With anticoincidence, the backgrounds for each geometry were lowered to values $1 / 3$ to $1 / 10$ of the previous over most of the energy range from 0.1 to $3 \mathrm{MeV}$. Compared with the $1 / 8$ in. crystal separation, the background counts in the energy-energy plane were lowered to $1 / 2$ to $1 / 9$ with the crystals 10 in. apart and were decreased even further when the "torso water bath" was between the crystals. These background reductions with the crystals separated 10 in. were, of course, due to the decreased probability of a single gamma-ray, or of two or more gamma rays produced from a cosmic ray shower, interacting with the two detectors simultaneously. The two peaks in the energy-energy background which occurred around Channels 15 and 22 (total energy loss of 1.47 and $2.225 \mathrm{MeV}$ ) when the detectors were together were the result of the scatter of a partially absorbed ${ }^{40} \mathrm{~K}$ or deuterium de-excitation gamma ray from one NaI crystal into the other.

The effect of the anticoincidence shielding and source geometry on the response of one of the single crystals is illustrated in Figure 99. With the detectors $1 / 8$ in. apart, the anticoincidence shielding reduces the Compton 


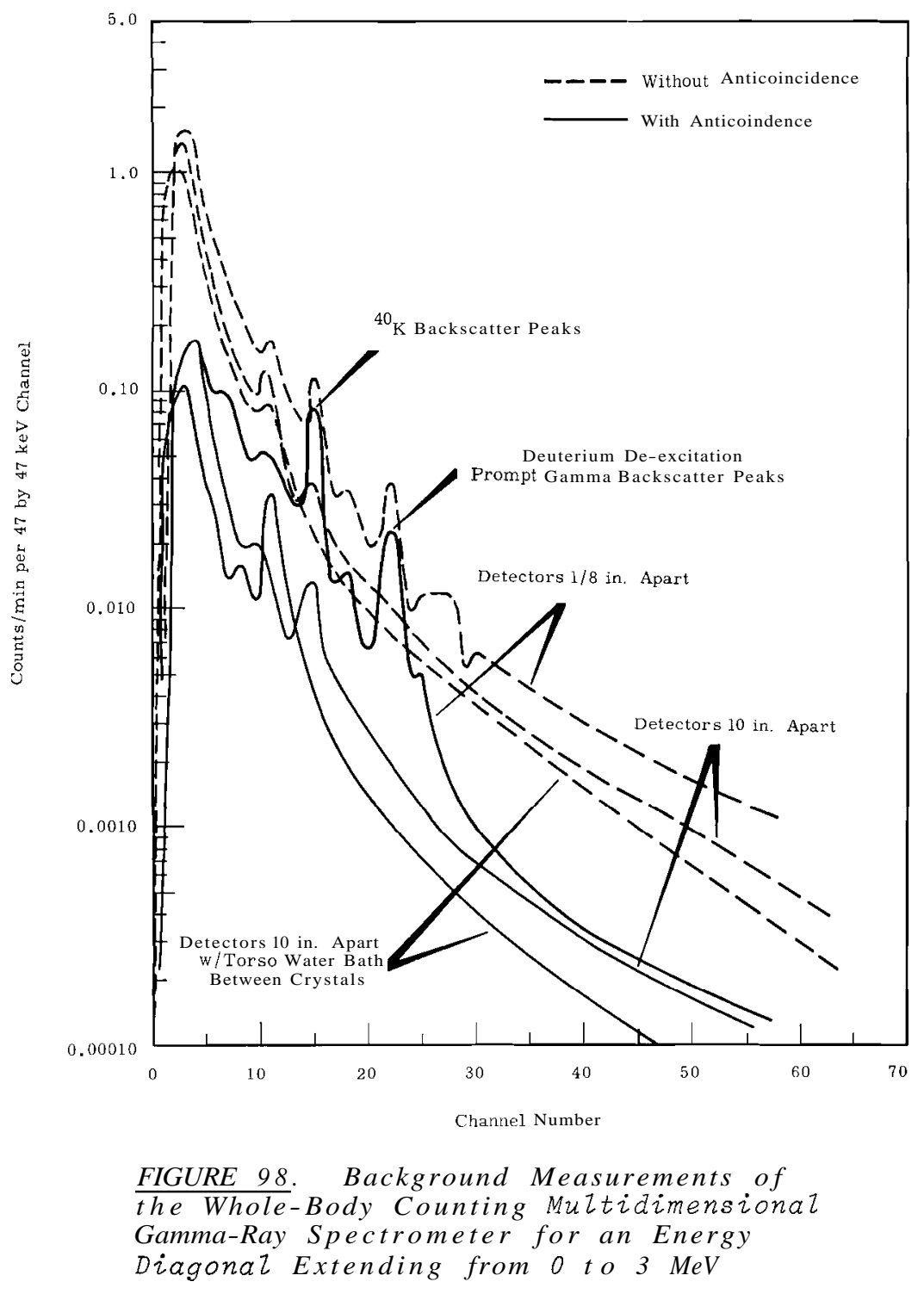

regions of a ${ }^{60}$ Co spectrum to $1 / 2$ to $1 / 5$ over most of the energy range and the photopeaks at 1.17 and $1.33 \mathrm{MeV}$ to 1/3. By separating the crystals 10 in. and centering the ${ }^{60}$ Co point source between the crystals, the coincidence counting efficiency is reduced to about $1 / 20$ to $1 / 25$ while the counting rates of the 1.17 and $1.33 \mathrm{MeV}$ gammarays are reduced to only about $1 / 2$, when compared with the system not operating with anticoincidence. Placing the "torso water bath" between the crystals, with the ${ }^{60}$ Co source suspended in the center of the bath produces an additional reduction to $1 / 3$ to $1 / 4$ in the coincidence peak and a 30 to $40 \%$ reduction in the single gamma-ray peaks. The probability for the two ${ }^{60}$ Co gamma rays being totally absorbed, one each in the separate detectors is about the same as for both being absorbed by one detector (see Figure 99). Thus, relatively low coincidence counting efficiencies are obtained in whole body counting. 


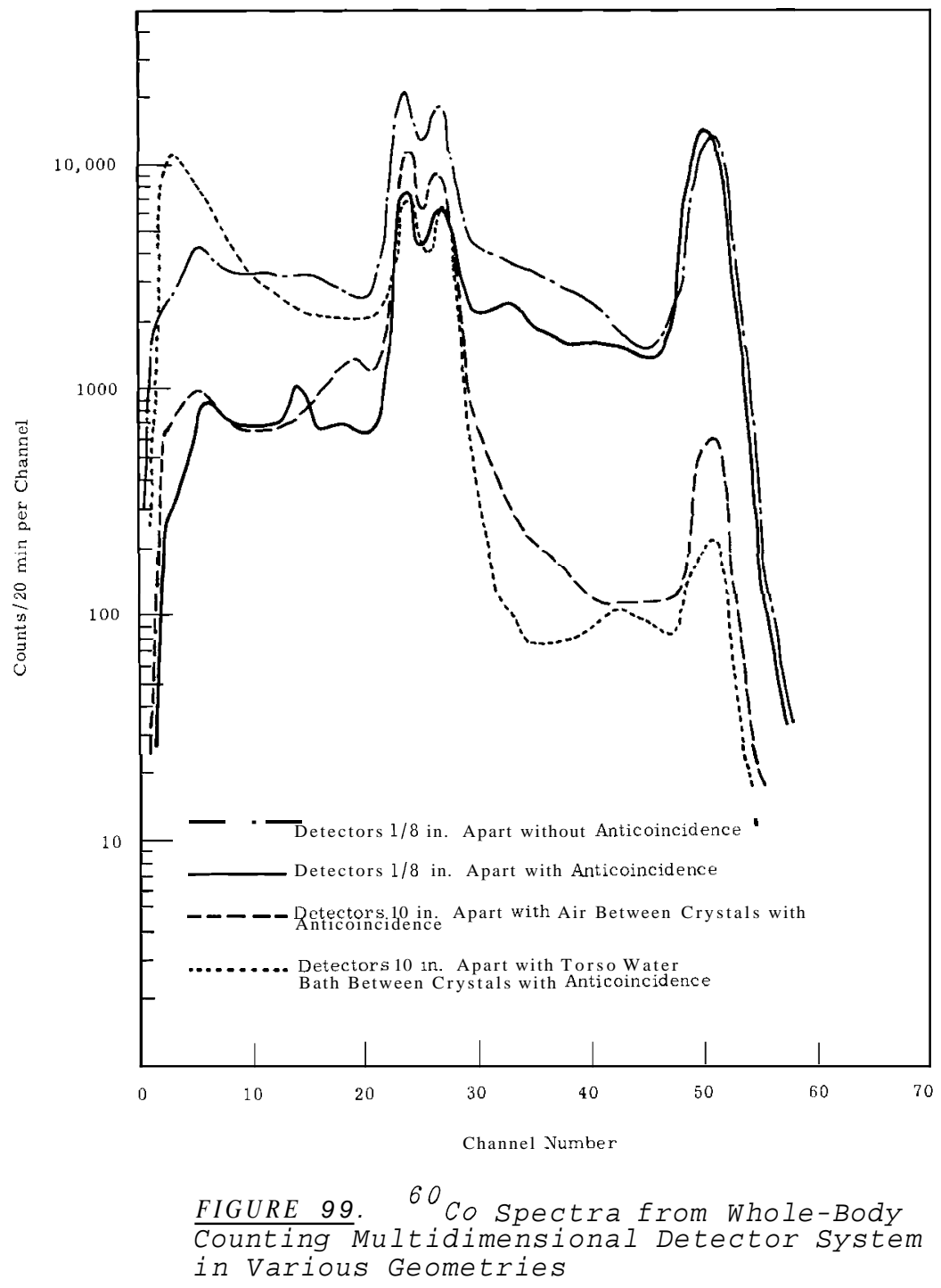

\section{Counting Efficiencies}

Table XLVI summarizes the photopeak counting efficiencies for ${ }^{51} \mathrm{Cr}$, ${ }^{65} \mathrm{Zn},{ }^{137} \mathrm{Cs},{ }^{60} \mathrm{Co}$, and ${ }^{226} \mathrm{Ra}$ a $\mathrm{t}$ crystal spacings of $1 / 8 \mathrm{in}$. and 10 in. For the 10 in. spacing the point source is centered between the crystals, and is in air for one set of measurements and in the "torso water bath" for the other set of measurements. The decrease in efficiency with geometry and absorption mater- ial i s evident. While the efficiency for a single gamma ray such as that of ${ }^{65} \mathrm{Zn} \quad(1.11 \mathrm{MeV})$ is only reduced to $1 / 10$ by going from a point source $1 / 8$ in. geometry to the "torso water bath" the efficiency for coincidence counting of ${ }^{60}$ Co dropped to about $1 / 90$ of its original value.

\section{$\underline{\text { Whole Body Counting }}$}

An evaluation of this multidimensional detector system as a whole 
TABLE XLVI. Counting Efficiency of the Whole Body Counting Multidimensional Gamma-Ray Spectrometer at Various Geometries with Anticoincidence

\begin{tabular}{|c|c|c|c|}
\hline$\underline{\text { Radionuclide }}$ & Geometry $(\mathrm{a})$ & $\begin{array}{l}\text { Counting Efficiency, } \\
\text { Single Gamma Rays } \\
\text { (b) }\end{array}$ & Counting Efficiency, Counts/min/nCi \\
\hline \multirow[t]{3}{*}{${ }^{51} \mathrm{Cr}$} & A & 147 & $\cdots$ \\
\hline & B & 42.4 & --- \\
\hline & $\mathrm{C}$ & 10.2 & $\cdots$ \\
\hline \multirow[t]{3}{*}{${ }^{65} \mathrm{zn}$} & A & 394 & -- \\
\hline & B & 84.9 & $-\cdots$ \\
\hline & C & 42.6 & $-\cdots$ \\
\hline \multirow[t]{3}{*}{${ }^{137} \mathrm{Cs}$} & A & 938 & $-\cdots$ \\
\hline & B & 215 & --- \\
\hline & $\mathrm{C}$ & 95.4 & -- \\
\hline \multirow[t]{3}{*}{${ }^{60} \mathrm{Co}$} & A & 131 & 168 \\
\hline & B & 191 & 8.00 \\
\hline & c & 117 & 1.83 \\
\hline \multirow[t]{3}{*}{$226_{\mathrm{Ra}}$} & A & -- & -.- \\
\hline & B & 35.1 & 3.34 \\
\hline & C & 22.5 & 0.95 \\
\hline \multicolumn{4}{|c|}{$\begin{aligned}{ }^{(a)} \mathrm{A}= & \text { Detectors } 1 / 8 \mathrm{in.} \text { apart with point source center between them } \\
\mathrm{B}= & \text { Detectors } 10 \mathrm{in} . \text { apart with point source center between them } \\
\mathrm{C}= & \text { Detectors } 10 \mathrm{in} . \text { apart with the "torso water bath" centered } \\
& \text { between the crystals }\end{aligned}$} \\
\hline
\end{tabular}

body counter was made by comparing its detection sensitivity with that of a single crystal with and without anticoincidence shielding and light piping (see Table XLVII). The detection sensitivities were measured by counting point sources of ${ }^{60} \mathrm{Co},{ }^{226} \mathrm{Ra}$, and 65 Zn which were centered in the "torso water bath." The "torso water bath" was centered between the two $93 / 8$ in. diam by 4 in. thick NaI(T1) crystals in the multidimensional system and centered directly under the $93 / 8$ in. diam by 4 in. thick $N a I(T 1)$ single crystal systems. All counting was performed in 4 in. lead caves. The sensitivities for measuring $0.10,0.50$, and $1.00 \mathrm{nCi}$ of each radionuclide during a 100 min count at the $90 \%$ confidence level was calculated from the counting efficiencies and backgrounds (including the contribution from a person) of each detector system (see Table XLVII). The sensitivity for measuring $0.10 \mathrm{nCi}$ of ${ }^{60} \mathrm{Co}$ or ${ }^{226} \mathrm{Ra}$ is nearly identical for each detector system. Although the backgrounds of the single crystal sysems were about 2 to 4 orders of magnitude higher than that of the multidimensional system, the coincidence counting efficiency of the latter was reduced, thus making the sensitivities of the sysems essentially equivalent. The 
TABLE XLVII. Comparison of Detecfion Sensitivities of Various Detector Systems for Whole Body Counting (a)

\begin{tabular}{|c|c|c|c|c|}
\hline \multirow[b]{2}{*}{ Radionuclide } & \multicolumn{4}{|c|}{$\begin{array}{c}\text { Detection Sensitivity in } \mathrm{nCi} \text { for a } 100 \text { min Count at } 90 \% \\
\text { Confidence Level }\end{array}$} \\
\hline & $\begin{array}{l}\text { Multidimensional System, } \\
\text { Two } 9 / 8 \text { in. by } 4 \text { in. } \\
\text { crystals in coincidence } \\
\text { with anticoincidence } \\
\text { shielding }\end{array}$ & $\begin{array}{l}\text { Single } 93 / 8 \text { in. } \\
\text { crystal with } \\
\text { anticoincidence } \\
\text { and light pipe }\end{array}$ & $\begin{array}{l}\text { Single } 9 \\
\text { crystal } \\
\text { anticoin } \\
\text { and ligh }\end{array}$ & $\begin{array}{l}93 / 8 \text { in } \\
\text { without } \\
\text { ncidence } \\
\text { ht pipe }\end{array}$ \\
\hline${ }^{60} \mathrm{Co}$ & $\begin{array}{l}0.10 \pm .04 \\
0.50 \pm .09 \\
1.00 \pm .12\end{array}$ & $\begin{array}{l}0.10 \pm .05 \\
0.50 \pm .05 \\
1.00 \pm .05\end{array}$ & $\begin{array}{l}0.10 \pm \\
0.50 \\
1.00 \pm\end{array}$ & $\begin{array}{l} \pm .05 \\
\pm .05 \\
\pm .05\end{array}$ \\
\hline${ }^{226} \mathrm{Ra}$ & $\begin{array}{l}0.10 \pm .09 \\
0.50 \pm .14 \\
1.00 \pm .19\end{array}$ & $\begin{array}{l}0.10 \pm .11 \\
0.50 \pm .11 \\
1.00 \pm .12\end{array}$ & 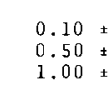 & $\begin{array}{ll} \pm & .14 \\
\pm & .14 \\
\pm & .14\end{array}$ \\
\hline${ }^{65} \mathrm{Zn}$ & - & $\begin{array}{l}0.10 \pm .08 \\
0.50 \pm .08 \\
1.00 \pm .08\end{array}$ & $\begin{array}{l}0.10 \pm \\
0.50 \mathrm{t} \\
1.00 \pm\end{array}$ & $\begin{array}{l} \pm .17 \\
\pm .17 \\
\pm \quad .17\end{array}$ \\
\hline
\end{tabular}

error in measuring $1.00 \mathrm{nCi}$ of ${ }^{60} \mathrm{Co}$ or ${ }^{226} \mathrm{Ra}$ is relatively less for the single crystal systems because their greater counting efficiencies allow higher counting rates with correspondingly lower standard deviations.

Although the background is reduced by anticoincidence shielding, this also cancels single gamma rays from a cascade where a second gamma ray from the cascade is seen by anticoincidence shield. Thus the counting efficiency for individual coincidence gamma rays is reduced. The benefit of anticoincidence shielding and light piping for single gamma ray detection is better observed by comparing the detection sensitivity of a non-coincidence gamma-ray emitting radionuclide, such as ${ }^{65} \mathrm{Zn}$. Here, the lower background afforded by the anticoincidence shielding and light pipe resulted in about a 2 fold improvement in the detection sensitivitiy for ${ }^{65} \mathrm{Zn}$. The detection sensitivities of other radionuclides could be expected to be increased by about the same amount. The sensitivities calculated in this study were based on the assumption that the radionuclide being measured was the only one present in the body (except for the natural ${ }^{40} \mathrm{~K}$ and some ${ }^{137} \mathrm{Cs}$ ). If other radionuclides were present their Compton interference could seriously hinder or prohibit the detection of smaller concentrations of other radionuclides which could be present.

Under these conditions multidimensional whole body counting could prove most useful. Since the Compton interference in multidimensional gamma-ray spectrometry is, in most cases, sma11, the detection sensitivities in Table XLVII would remain essentially constant even if other radionuclides were also present in the body. However, the sensitivities of the single crystal systems, especially the non-anticoincidence shielded detector, could be seriously reduced.

A few persons were counted for 100 min in the multidimensional whole body 
counting detector system. The only radionuclides detected were ${ }^{40} \mathrm{~K}$, ${ }^{137} \mathrm{Cs}$ (from fallout), and ${ }^{65} \mathrm{Zn}$ (from drinking Columbia River water). The concentrations of coincidence gammaray emitting radionuclides in their bodies were therefore lower than the detection limits stated in Table XLVII No opportunity was available to count persons who contained relatively high concentrations of a radionuclide mixture in the body.

\section{Discussion and Conclusions}

Multidimensional gamma-ray spectrometry may prove very useful in wholebody counting, especially in the cases in which a relatively high concentration of a complex mixture of radionuclides is present. Although the coincidence counting efficiency of this system is greatly reduced by separating the crystals to allow for whole-body counting, the detection limits of most radionuclides which decay with cascade gamma rays are still quite reasonable since the background in the energy-energy plane is extremely low for counts as long as $100 \mathrm{~min}$. These detector systems also offer several advantages as normal gamma-ray spectrometer wholebody counters. The anticoincidence shielding significantly lowers the background and Compton response of each crystal thus increasing their sensitivities. Also, a twin crystal system doubles the counting efficiency.

The sensitivity of the present detector system is just marginal compared to that of a single crystal. Since the coincidence counting efficiency increases rapidly with crystal size, coincidence counting sensitivities would increase relative to single crystal sensitivities as the crystal sizes were increased beyond those of the present system.

A similar detector system is near completion which consists of two $11 \mathrm{in}$. diam by 6 in. thick NaI(T1) crystals each fitted with 4 in. thick pure NaI light pipes and surrounded by 36 in. diam plastic phosphor anticoincidence shielding. This new system will also be evaluated as a whole-body counter.

\section{AN ANTICOINCIDENCE-SHIELDED Ge ( $\mathrm{L} i$ ) GAMMA-RAY SPECTROMETER-M. W. Hill}

An anticoincidence-shielded gammaray spectrometer was assembled using a Ge(Li) diode as the primary detector and a large $26 \mathrm{in}$. diam by $24 \mathrm{in}$. thick plastic phosphor as the anticoincidence shielding. The diode was housed at the end of a vacuum chamber containing a long cold finger and inserted in the plastic phosphor through a 2 in. diam hole. Pulses from the Ge(Li) detector were fed through an Ortec 101-XL preamplifier into a RIDL 34-12 pulse height analyzer. Pulses from the plastic phosphor shield were fed into the anticoincidence gate of the analyzer. The signal lead capacitance resulting from the long signal lead was $9 \mathrm{pF}$. With this arrangement, reduction at the Compton edge and backscatter peak of ${ }^{137} \mathrm{Cs}$ was 11.5 and 6.3 respectively for a $1 \mathrm{~cm}^{2}$ by $1 \mathrm{~mm} \mathrm{Ge(Li)} \mathrm{detector.} \mathrm{For} \mathrm{those} \mathrm{ra-}$ dionuclides having cascading gamma rays, both the intensity of the Compton 
continuum and the photoelectric peak were reduced; however, the intensity of the photopeak relative to the Comp- ton edge was still enhanced. For ${ }^{134} \mathrm{Cs}$, the reduction of the Compton edge was to $1 / 20$ while that of the $605 \mathrm{keV}$ photopeak was to 1/12.

REDESIGN AND APPLICATON OF AN ANTICOINCIDENCE-SHIELDED Ge ( $\mathrm{i} i$ ) GAMMA-RAY SPECTROMETER-C. W. Thomas

The anticoincidence-shielded gammaray spectrometer originally designed and constructed by M. W. Hil1 (147) utilized a $\mathrm{Ge}(\mathrm{Li})$ diode as the primary detector and a large 26 in. diam by 24 in. thick plastic phosphor as the anticoincidence shielding. The diode was housed at the end of a long 19 in. vacuum chamber containing a cold finger which was inserted in the plastic phosphor through a 2 in. diam hole. To change samples it was necessary to remove the long vacuum housing from the plastic phosphor by means of a counterweighted pulley system after disconnecting the preamplifier and the vac-ion pump. During such a period of time some temperature and pressure irregularities were encountered as well as gain instability after reassembling the system. The uncertainty of gain shift when determining unknown radionuclides required a more stable system. I t was also noted that when operating the system under these conditions, some preamplifier noise was encountered. Over a period of 2 weeks use the resolution of this system had changed from $6.5 \mathrm{keV}$ (FWHM) to 14.1 keV (FWHM). Measurements showed that the loss was due to the preamplifier, not to the $4 \mathrm{~cm}^{2}$ diam by $5 \mathrm{~mm}$ depletiondepth diode.

To improve the design, a sample port was designed to enter through the side of the plastic phosphor. A long channel $1 / 2$ in. thick by 2 in. wide by 12 in. long was milled out of the underside of the top section of plastic phosphor so that it connected with the 2 in. diam hole. A $30 \mathrm{mil}$ aluminum 1 ine $\mathrm{r}$ was made to line both the hole and the sample trough. Samples were inserted using a long Lucite paddle.

The system has been in constant use for measuring the radionuclide composition of a variety of samples. Figure 100 shows the response of the system, with and without anticoincidence shield-

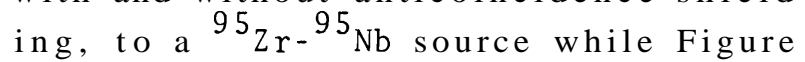
101 shows the response to a source of 1 day old non-volatile fission products. Although the resolution with this system does not compare with that of diodes using FET preamplifiers, the effect of the anticoincidence shielding does give a photopeak-to-Compton ratio which is comparable to that of the high resolution systems. Figure 102 compares the response of this system to that of a NaI(T1) detector. With the exception of cesium the isotopes mentioned above are present in reactor tube film and the system has been used effectively in analyzing samples of that type.

In addition to measuring samples of high activity, low level samples have been measured quite effectively. Figure 103 is a spectrum of ashed wolf muscle showing the natural ${ }^{40} \mathrm{~K}$ and the 
fallout isotope ${ }^{137}$ Cs. Figure 104 is the spectrum of an air filter sample showing the fallout isotopes ${ }^{144} \mathrm{Ce}$, ${ }^{141} \mathrm{Ce}, 125_{\mathrm{Sb}},{ }^{131} \mathrm{I},{ }^{7} \mathrm{Be}, 103_{\mathrm{Ru}},{ }^{106} \mathrm{Ru}$, ${ }^{137} \mathrm{Cs},{ }^{95} \mathrm{Zr}-{ }^{95} \mathrm{Nb}$, and ${ }^{54} \mathrm{Mn}$. From these results it is apparent that even a small Ge(Li)diode is useful in some low level counting applications.
Additional modifications are underway. An FET preamplifier has been designed so that the input stage will be nounted near the diode thus increasing the resolution of the system. Larger diodes of the coaxial type are also planned for incorporation into the system.

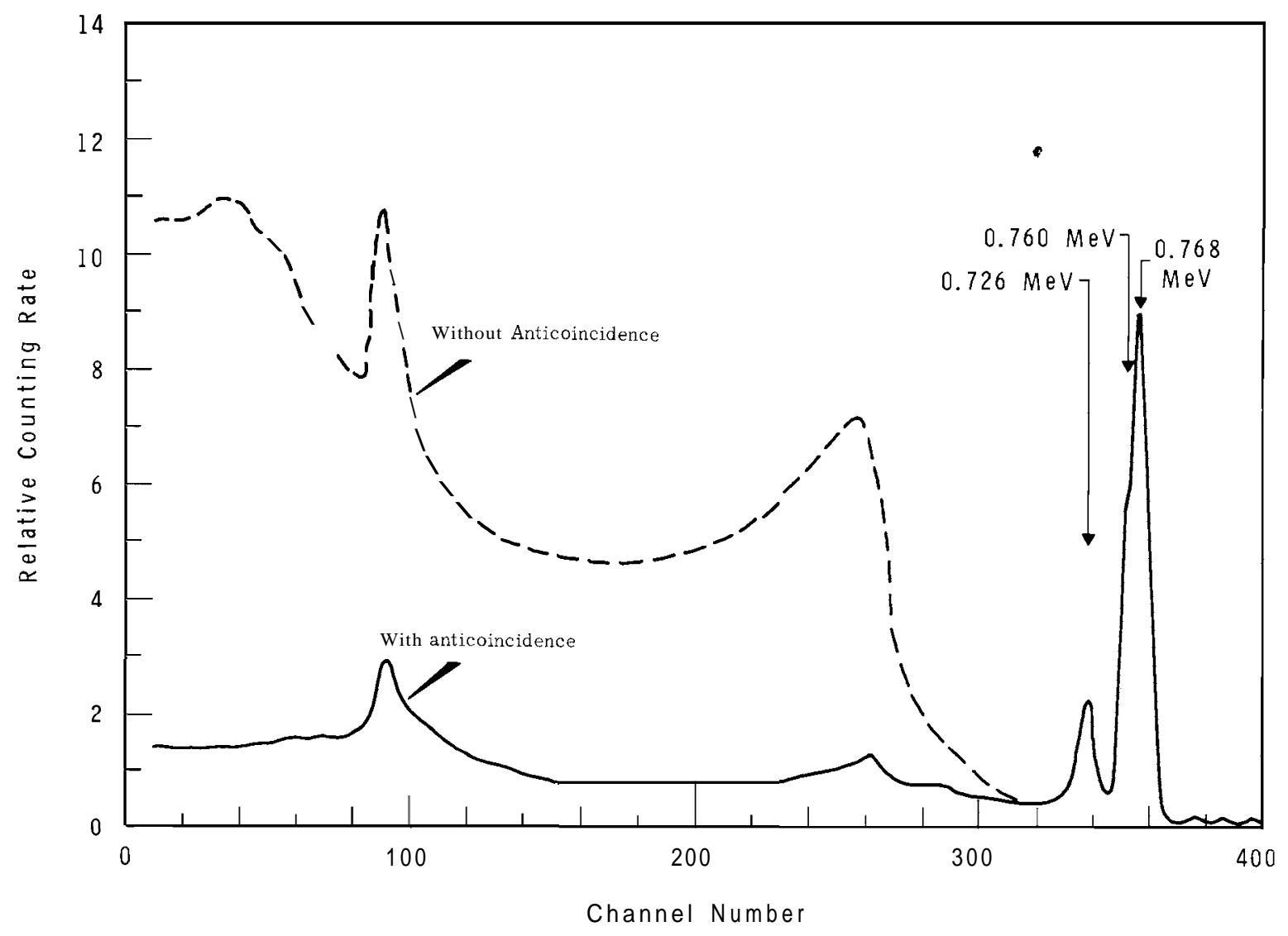

FIGURE 100. Response of an Anticoincidence Shielded $5 \mathrm{~mm}$ Depletion Depth Ge(Li) Diode to a ${ }^{95} \mathrm{Zr}-^{95} \mathrm{Nb}$ Source 


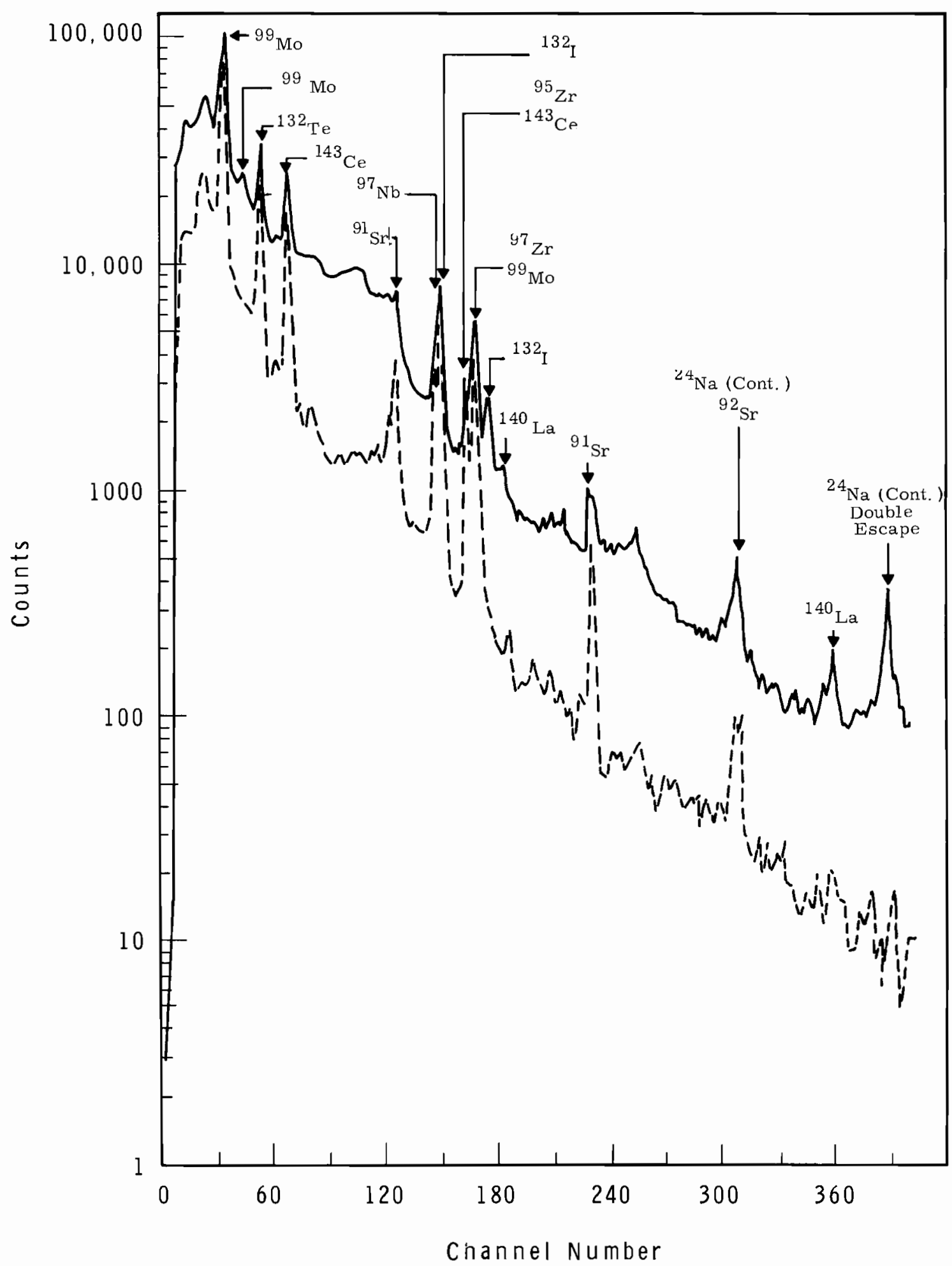

EIGURE 101. One Day OZd Nonvolatile

Fission Product Counted with and

Without Anticoincidence Shiezding 


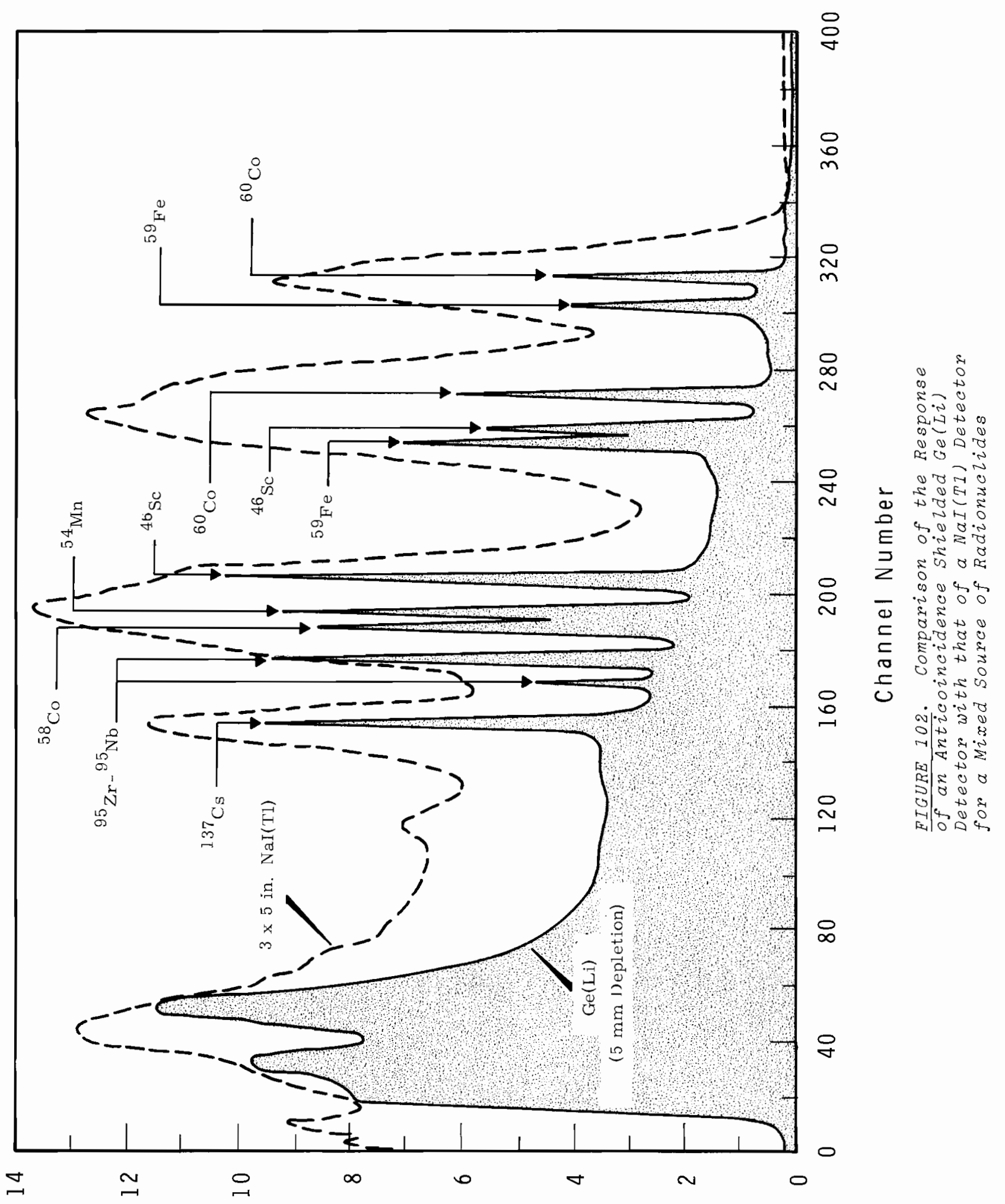

әfey bu!funoJ ә八!feləy 


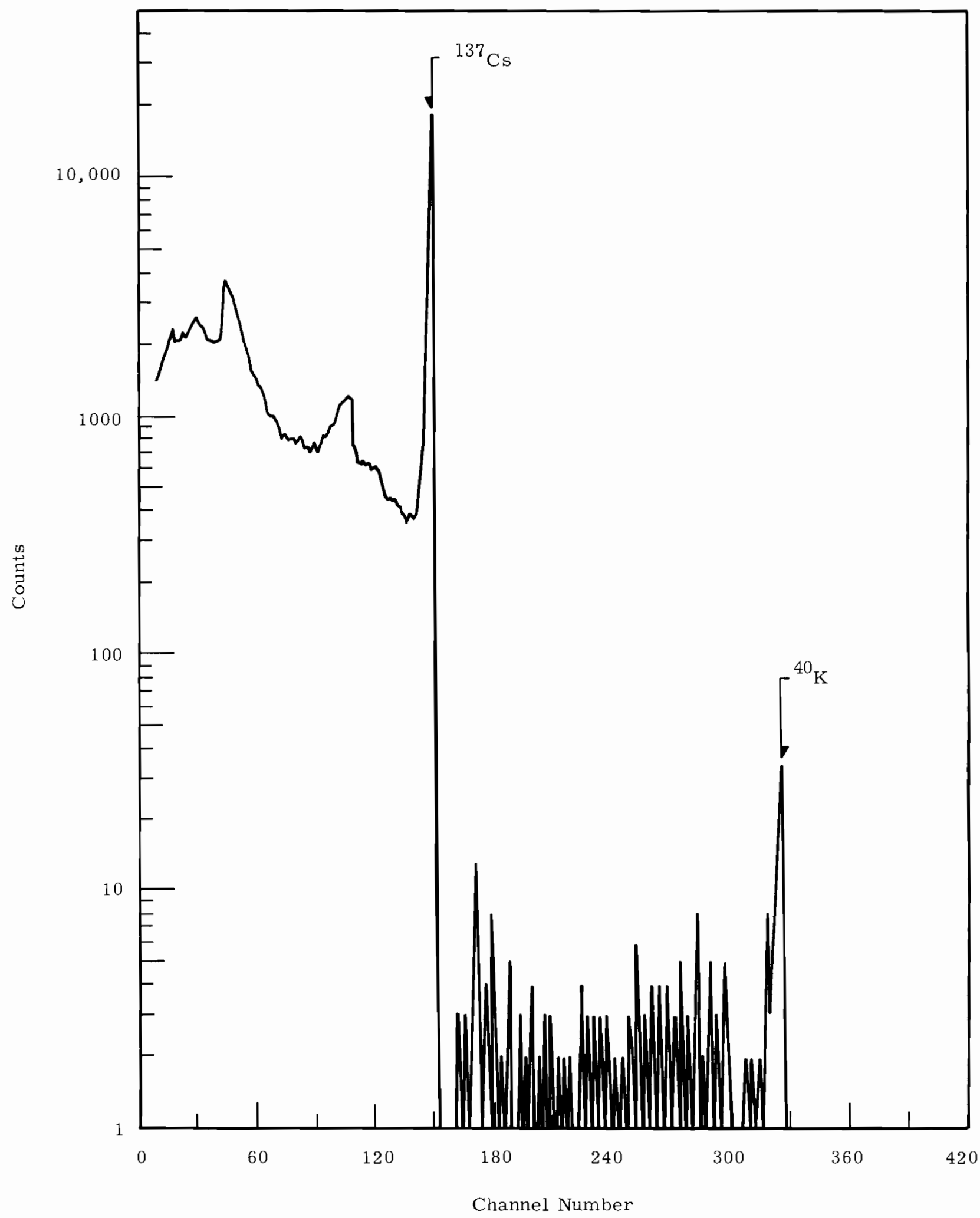

FIGURE $\frac{103}{\text { Muscie }}$ Ash $\quad$ Radionuclides - Wolf 


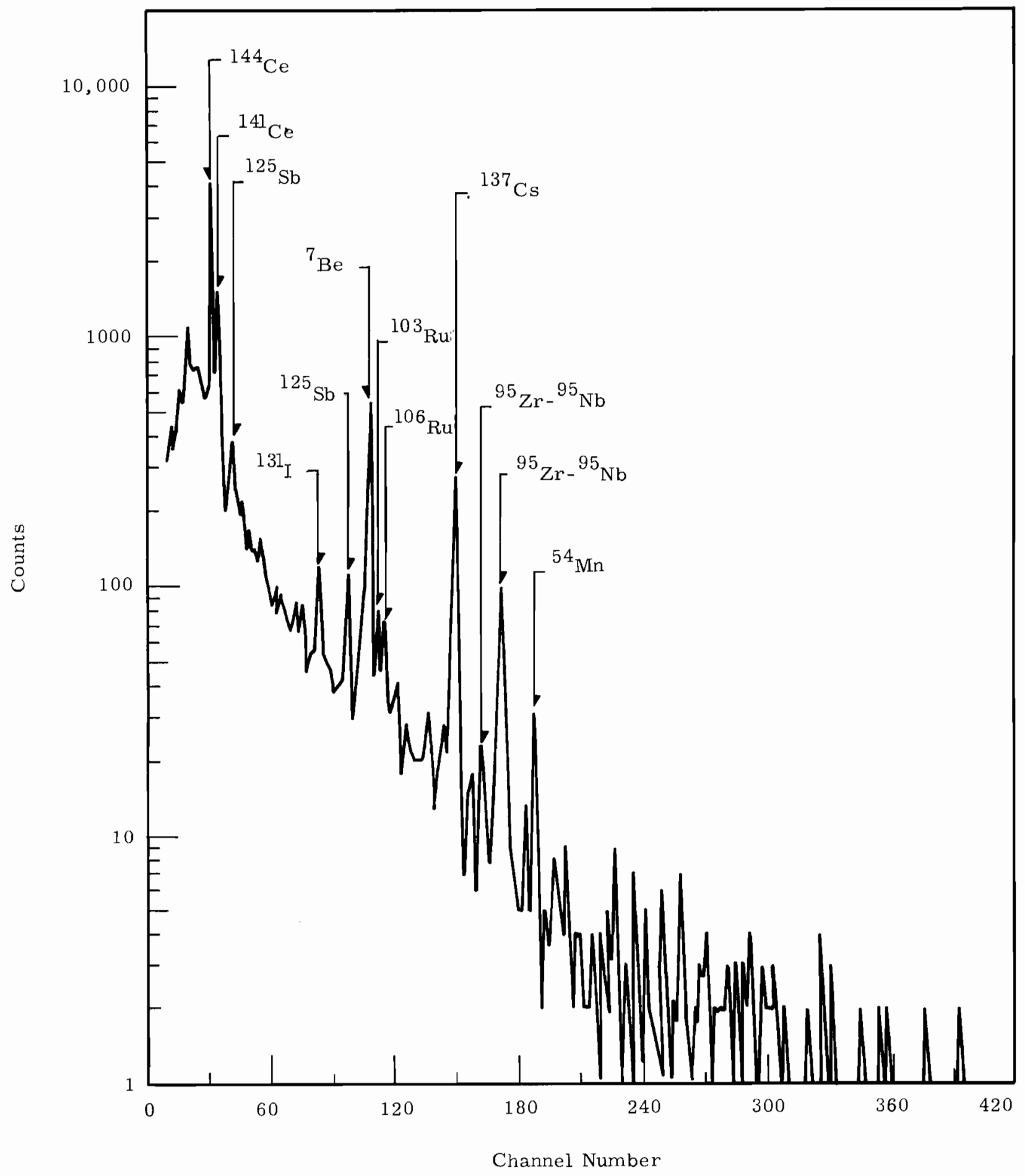

FIGURE 104. Air Filter sample showing 
EVALUATION OF RADIOCHEMICAL METHODS FOR BIOLOGICAL PURPOSES-C. W. ThOMAS, W. B. Silker, and J. D. Ludwick

A program to evaluate radiochemical methods critically for use with environmental and biological samples was continued. The following procedures for determining radionuclide concentrations in the given matrices were investigated, tested, and written in standard form by this laboratory: gold in water and urine; barium in water and urine; cesium in water and urine; thor- ium in water and urine; neptunium in water, urine, air, tissue, and excreta; uranium in air, tissue, and excreta; americium in air and tissue; plutonium in air, tissue, and excreta. In addition, a gamma-ray spectrometric method was evaluated for use on all matrices for gamma-ray emitting radionuclides. A compilation of the standard tested methods will be completed for use by AEC contractors on July 1, 1966.

DETERMINATION OF ELEMENTS IN WHEAT GRAIN BY NEUTRON ACTIVATION ANALYSIS AND MULTIDIMENSIONAL GAMMA-RAY SPECTROMETRY-W. A. Haller

A method was devised for simultaneous determination of many elements in wheat grain. With the use of neutron activation and a multidimensional gamma-ray spectrometer, simultaneous determination of the elements may be made by direct counting following appropriate decay periods or following minimal chemica1 manipulations. The need for involved chemical separations for each element is thereby elimated. The elements determined include manganese, sodium, potassium, copper, bromine, zinc, iron, cobalt, antimony, cesium, and scandium. The concentrations found agreed quite closely with those obtained by others using the more conventional wet chemistry techniques.

\section{SELECTIVE AND SENSITIVE ANALYSIS OF ACTIVATION PRODUCTS BY MULTIDIMENSIONAL GAMMA-RAY SPECTROMETRY $(98)_{-R}$. W. Perkins and D. E. Robertson}

Gamma-ray spectrometry detector systems have been developed for use with multidimensional analyzers which permit an extremely sensitive and selective analysis of complex radionuclide mixtures. With these systems, use is made of the individual gamma-ray decay characteristic of each radionuclide for identification and measurement. This is accomplished by sandwiching the sample between two detectors and cancelling those events not totally absorbed in these detectors with a third annular detector which serves as an anticoincidence guard. The sample can be held in a fourth annular discshaped detector which allows the sample to be seen by the two principal detectors but which intercepts and cancels most of the degraded 
photons which would be scattered between them. The principal detectors are shielded from the gamma-ray radiation originating in their phototubes with light pipes which provide a substantial background reduction. Applications of these instruments include the direct measurement of atomic-weapons-produced radionuclide and cosmic-rayproduced activation products in water, soil, and biological materials.

GAMMA-RAY BACKGROUND STUDIES FOR NaI(T1) AND PLASTIC DETECTORS-C. W. ThOmas

\begin{abstract}
Reduction in the background seen by a gamma-ray detector has been accomplished in a series of steps during the past 15 years. Initially, the reduction was attained by using massive shields such as lead. This was followed by utilizing low-activity lead and copper-cadmium liners in the cover. The latter reduced the low energy backscatter radiation. A major advance was made in background reduction when anticoincidence ring arrays were used to shield the main detector. Recently, large plastic phosphors or NaI(T1) crystals have been used to replace the ring arrays. During this period of time larger and higher resolution primary detectors have been made available. The combined effect of these crystals and the anticoincidence shielding was apparent. Identification of the peaks followed by appropriate action has been a valuable method used in reducing the background. For example, ${ }^{40} \mathrm{~K}$, which originated at the phototube, has been attenuated by using light pipes to separate the detector from the tube.

Certain photopeaks have recently been shown to be the result of an interaction between neutrons and the plastic phosphors used for anticoincidence shielding. The neutrons are
\end{abstract}

probably generated by the reaction of cosmic rays with the lead shielding. The most obvious recognizable photopeak is the $2.22 \mathrm{MeV}$ prompt gamma ray resulting from the reaction of the thermalized evaporative neutrons with the hydrogen in the plastic phosphor. Figure 105 shows the background spectra of a 9 by 9 in. NaI(TI) crystal with and without a paraffin blanket; these were taken in a $4 \mathrm{in.}$ thick lead cave. The normal background spectrum showed small photopeaks at $0.5,1.46$, and 3.1 and a relatively large peak at $6.6 \mathrm{MeV}$. A second background spectrum taken with the crystal surrounded by $11 / 2$ in. of paraffin illustrated that the hydrogen prompt gamma of $2.22 \mathrm{MeV}$ also increased under these conditions. Therefore, a reaction similar to that which produced the hydrogen prompt gamma may introduce the $6.6 \mathrm{MeV}$ photopeak. The plastic phosphor background shown in Figure 106 contained the $2.22 \mathrm{MeV}$ hydrogen prompt gamma but not the $6.6 \mathrm{MeV}$ photopeak. Thus, since similar caves were used for the plastic and for the sodium iodide detectors, it was concluded that the $6.6 \mathrm{MeV}$ photopeak was produced by evaporative neutron bombardment of the sodium iodide assembly.

Figure 107 depicts the background spectrum from 0 to $11 \mathrm{MeV}$ using a 


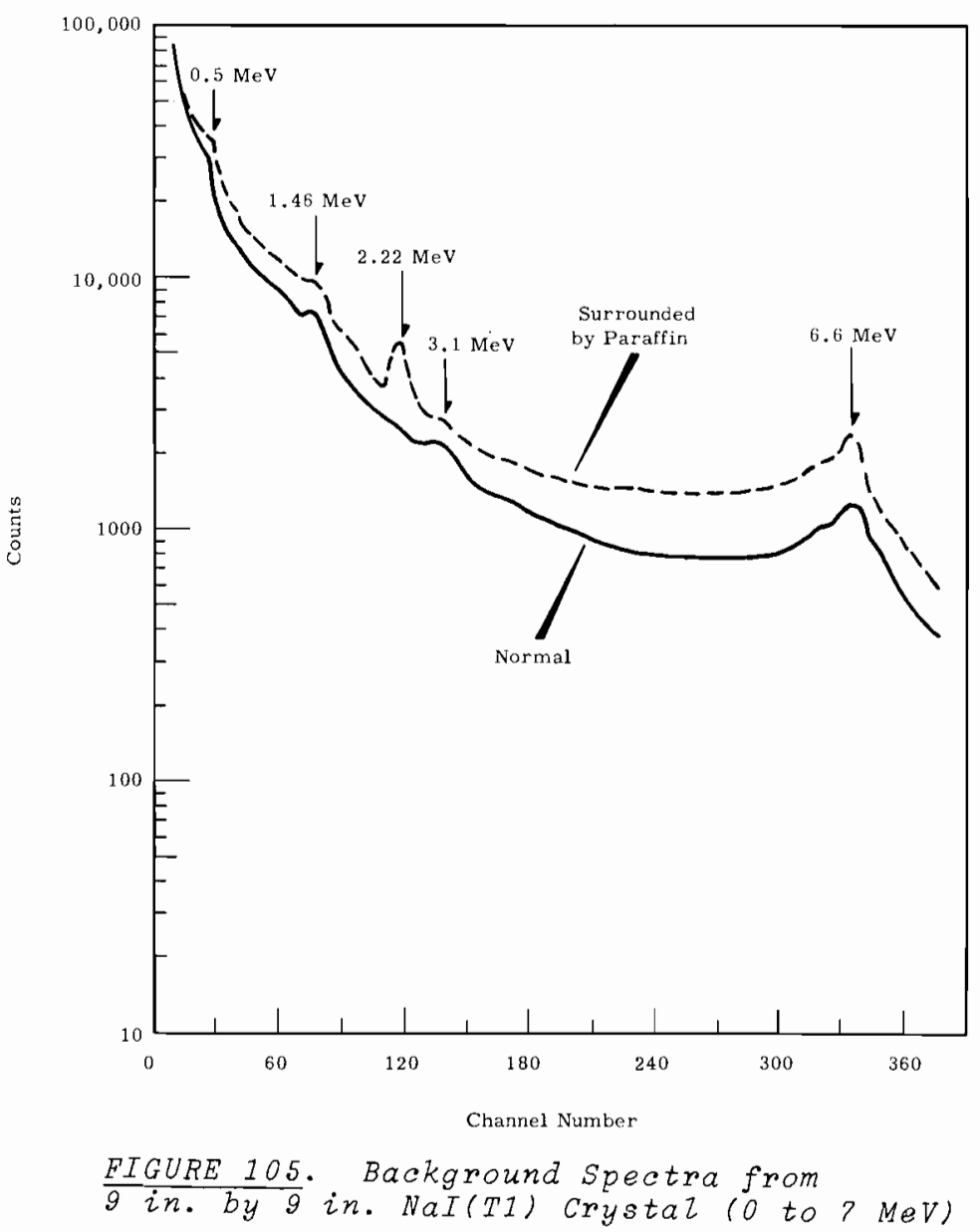

the 9 in. diam by 4 in. thick $\mathrm{NaI}$ (T1) crystals arranged in coincidence.

Two spectra were recorded, one with the crystals separated 11 in. apart, the other with the crystals on $1 y 17 / 8$ in. apart. Both spectra show similar photopeaks; however, the intensities varied depending on the distance separating the crystals. The difference in the peaks indicates that the main detector to exposed lead surface affects these photopeaks in various ways. The $6.6 \mathrm{MeV}$ photopeak doubled in intensity while the effect on the other photopeaks was much less. The slight increase for the $1.46,2.22$, and 3.1 $\mathrm{MeV}$ photopeaks appeared to be due to a higher Compton level resulting mainly from the $6.6 \mathrm{MeV}$ gamma ray.

Figure 108 is a background spectrum from 0 to approximately $100 \mathrm{MeV}$ which was recorded using the plastic phosphor. The large high energy photopeak at approximately $65 \mathrm{MeV}$ is believed to be due to the decay products of mesons. The particular reaction is presently being sought.

In conclusion, it appears that most of the photopeaks which are now present in the background are due to either meson decay or neutron reactions, which are produced by the interaction of cosmic rays with the lead shielding. 


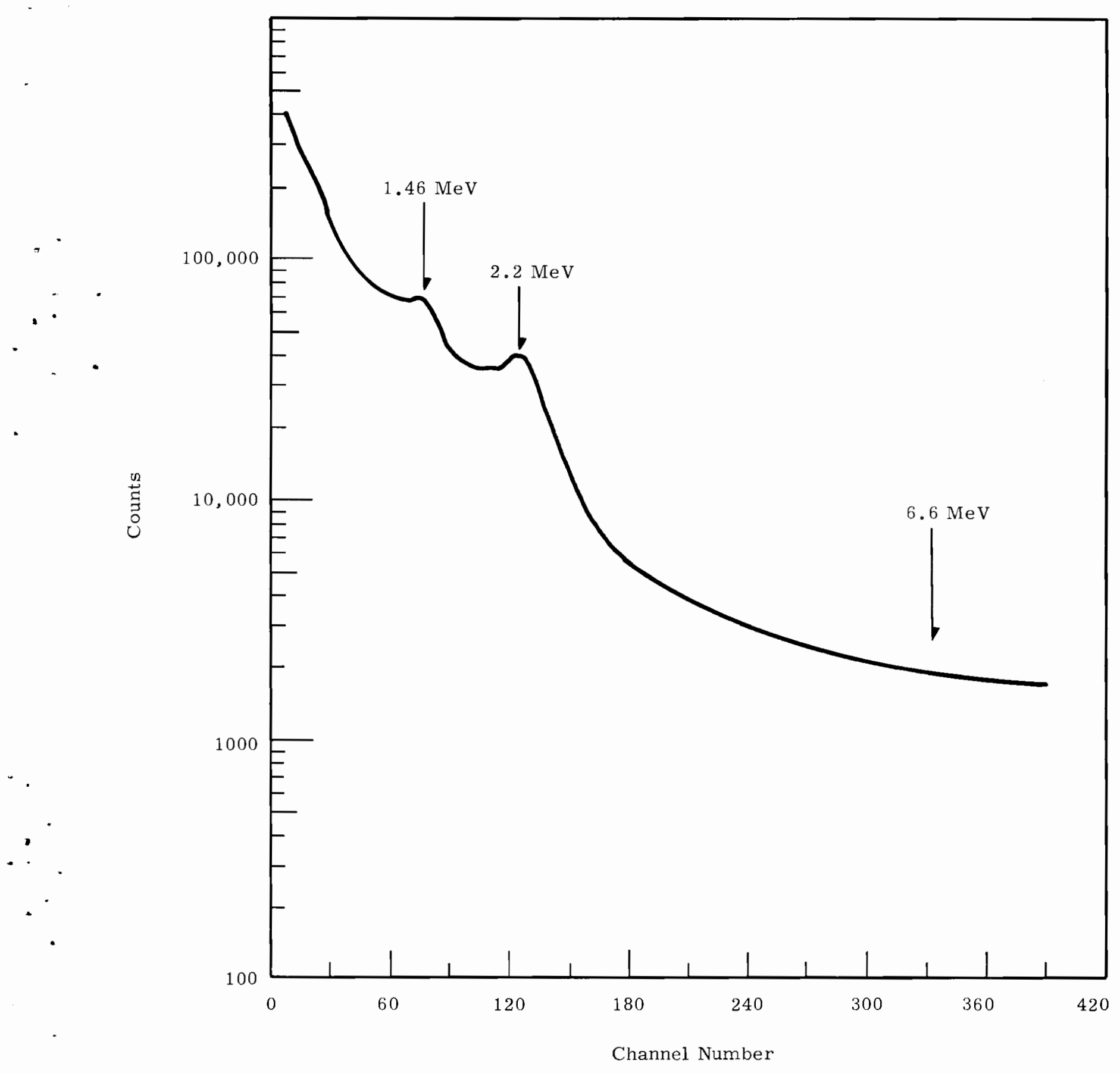

FIGURE 106. Background from Plastic 


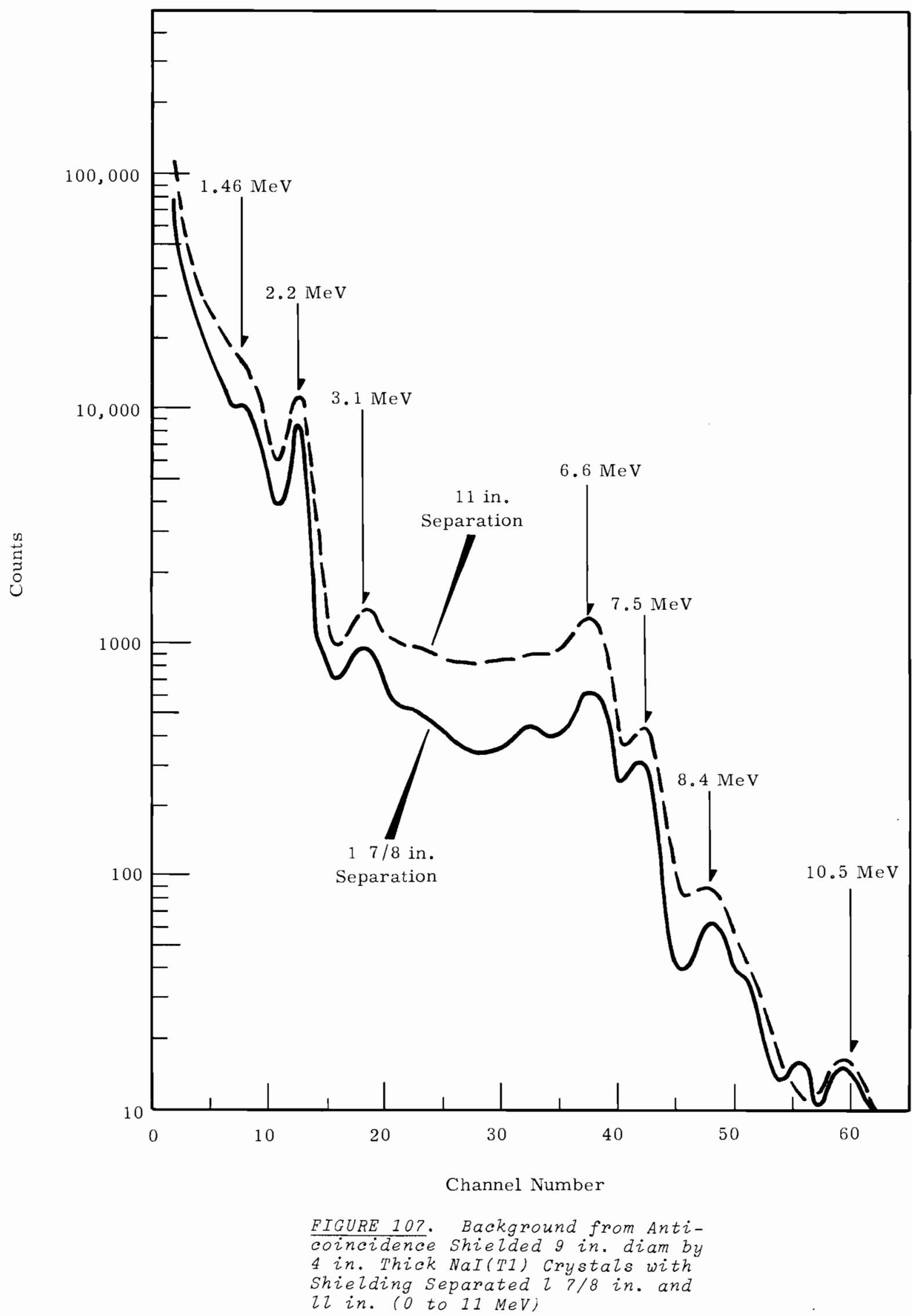




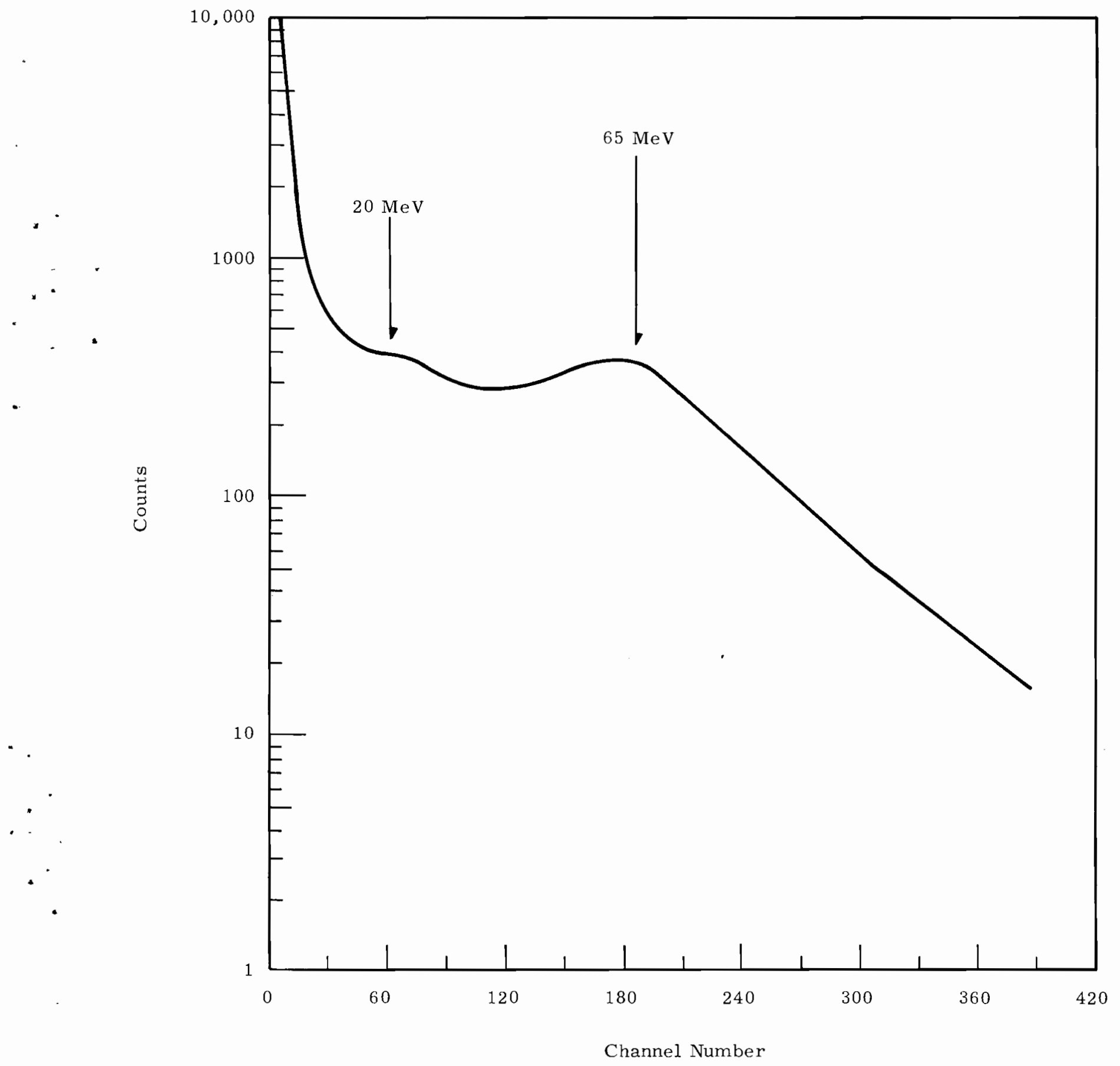

FIGURE 108. Background from Plastic 
GAMMA-RAY TRANSITIONS IN ${ }^{58} \mathrm{Fe}-\mathrm{M}$. W. Hill

The decay of ${ }^{58}$ Co has been studied by several investigators. ${ }^{(48-152)}$. A1though the decay scheme is extremely simple (see Figure 109), certain discrepancies exist between the values reported for the energies and intensities of the gamma-ray transitions in ${ }^{58} \mathrm{Fe}$. These discrepancies are primarily brought about by the small energy difference between the two cascading gamma rays and by the small percentage of decays leading to the second excited state. Frauenfelder et al. concluded from their studies that the energy difference between the two cascading gamma rays is less than 10 $\mathrm{keV}$, and that the energy of the second excited state is $1.62 \mathrm{MeV}$. MacArthur et al., using a beta-ray spectrometer found the energies of the cascading gamma rays to be $810.48 \pm 0.1 \mathrm{keV}$ and $864.8 \pm 1.0 \mathrm{keV}$. From gammagamma coincidence studies they found

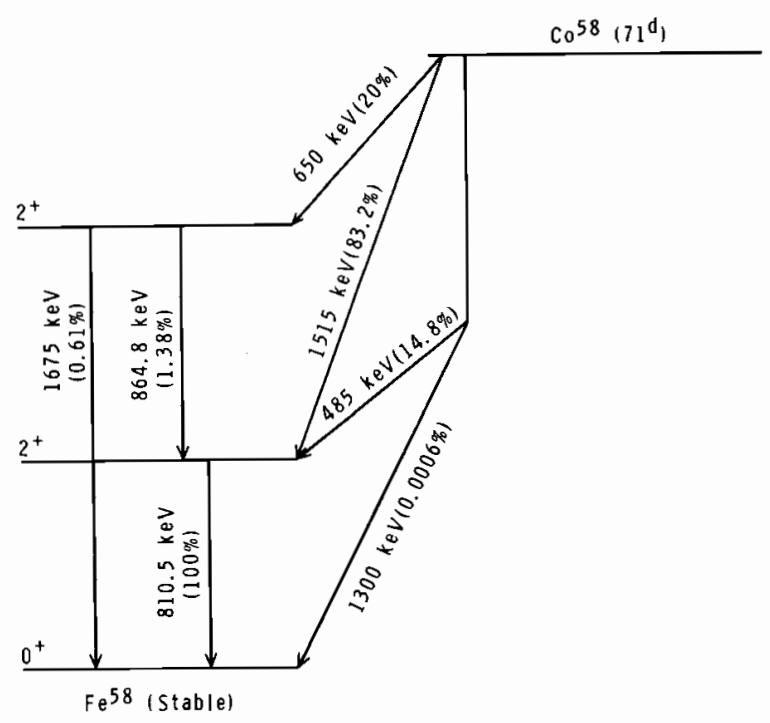

FIGURE 109. The Decay Scheme of ${ }^{58} \mathrm{Co}$ According to Malmskog the $865 \mathrm{keV}$ transition to be $1.20 \pm$ $0.06 \%$ as intense as the $810 \mathrm{keV}$ transition. Scintillation measurements placed the energy of the crossover transition at $1673 \pm 10 \mathrm{keV}(0.66 \pm$ $0.05 \%)$. The decay scheme according to Malmskog is shown in Figure 109. The energies of the gamma transitions are those of MacArthur et al. The intensities are those which Malmskog found from gamma-gamma coincidence measurements capable of distinguishing between chance and true coincidences. It seemed obvious that a direct measurement of the energies and intensities of all three gamma rays could easily be made with a lithium-drifted germanium detector.

The measurements reported here were made with three different sources of ${ }^{58}$ Co containing different amounts of ${ }^{57} \mathrm{Co}$ and ${ }^{60} \mathrm{Co}$ impurities. The spectra which are shown were obtained with a sample containing $0.4 \%{ }^{57}$ Co and $0.4 \%$ ${ }^{60}$ Co.

Three different detectors with active volumes of $1 \mathrm{~cm}^{2}$ by $1 \mathrm{~mm}, 1 \mathrm{~cm}^{2}$ by $2 \mathrm{~mm}$, and $4 \mathrm{~cm}^{2}$ by $5 \mathrm{~mm}$ were used during the course of the studies. The spectra shown in Figure 110 and Figure 111 were taken with the $1 \mathrm{~cm}^{2} \mathrm{x}$ $2 \mathrm{~mm}$ detector which had a resolution of $4.0 \mathrm{keV}$ at $662 \mathrm{keV}$.

Figure 110 shows the complete spectrum of ${ }^{58} \mathrm{Co}$. Similar spectra were obtained with each of the three detectors. Energies were determined to within $\pm 1.0 \mathrm{keV}$ by comparison with energy standards. Intensities were determined from efficiency curves established for each 


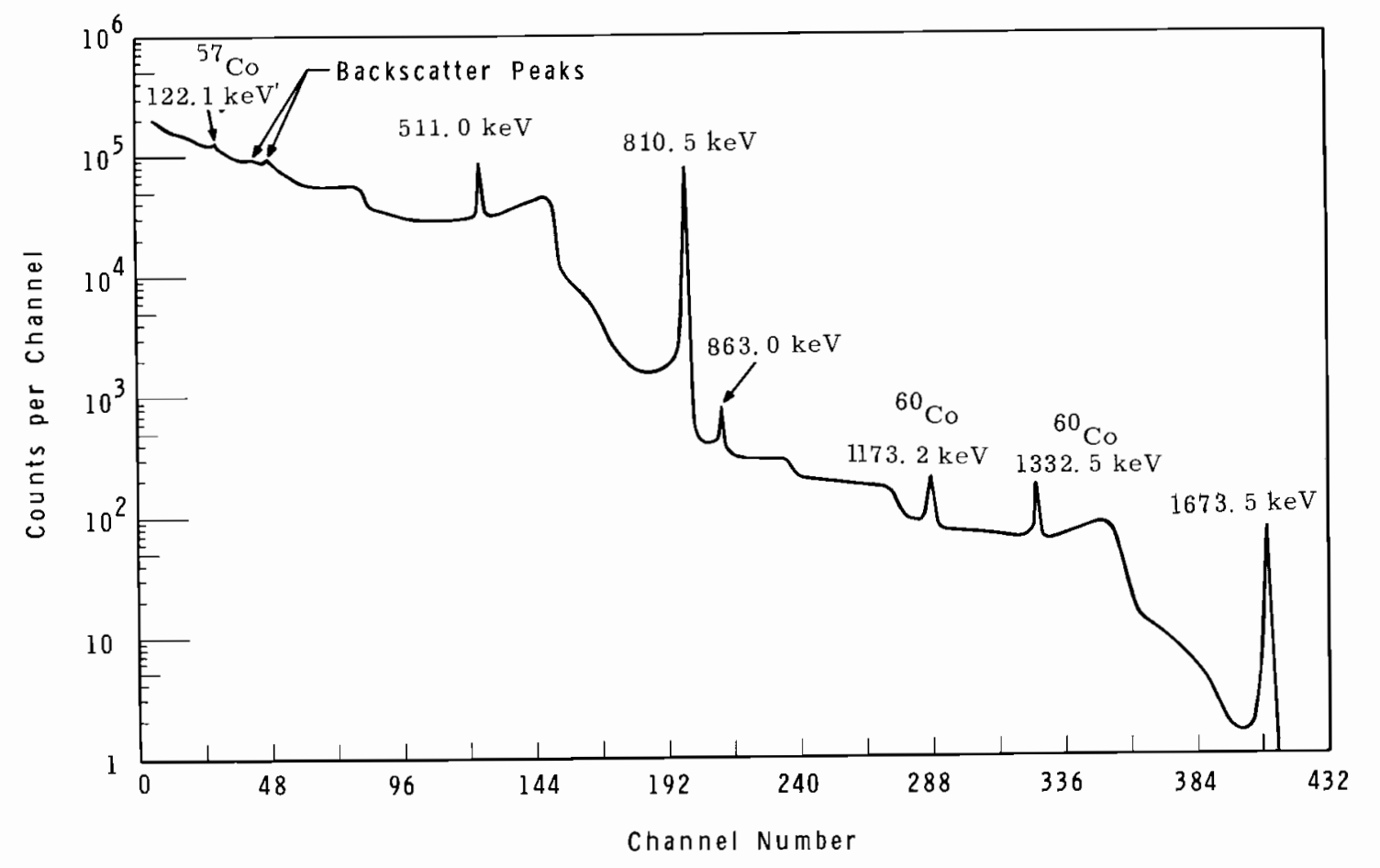

EIGURE 110. Gamma-Ray Spectrum of 58 To Taken with a Lithium-Drifted Germanium Detector - Small Amounts of $57 \mathrm{Co}$ and $60 \mathrm{Co}$ Were Present as Impurities

detector. Agreement between efficiencies obtained with the three detectors was wel1 within experimental and statistical errors.

To obtain a more accurate measurement of the energies and relative intensities of the two cascading gamma rays, the bias and gain were adjusted to give the spectrum shown in Figure 111. Although only two energy standards were needed, the third provided a check on the linearity. Uncertainties in peak location and any nonlinearity in response amounted to less than $0.2 \mathrm{keV}$.

The energies and intensities of the gamma rays emitted in the decay of ${ }^{58}$ Co were found to be $810.5 \pm 0.3$ $\mathrm{keV}(99.3 \%), 863.0 \pm 0.3 \mathrm{keV}(0.77 \pm$ $0.04 \%)$, and $1674.1 \pm 1.0 \mathrm{keV}(0.68 \pm$ $0.05 \%)$. The energy difference of $52.5 \pm 0.2 \mathrm{keV}$ found from Figure 111 between the $810.5 \mathrm{keV}$ and $863.0 \mathrm{keV}$ gamma rays is considered to be more accurate than the absolute energy found for either. The energy of the $1674 \mathrm{keV}$ gamma ray was not determined more precisely because it is obviously the crossover transition. Its energy is therefore $1673.5 \pm 0.4 \mathrm{keV}$.

The value shown for the intensity of the $863 \mathrm{keV}$ transition differs considerably from previously reported values. However, because of the 
direct nature of the measurement and the fact that the intensities obtained by using three different detectors and three different samples agreed to within $3 \%$, it is felt that the quoted limits of error are conservative. A measurement of this intensity was also made with an anticoincidence-shielded multi- dimensional gamma-ray spectrometer which stores the single spectrum as well as the coincidence spectrum. (102) By correcting the coincidence spectrum in the energy-energy plane for chance coincidences, a value of $0.8 \pm 0.2 \%$ was obtained for the intensity of the $863 \mathrm{keV}$ gamma ray.
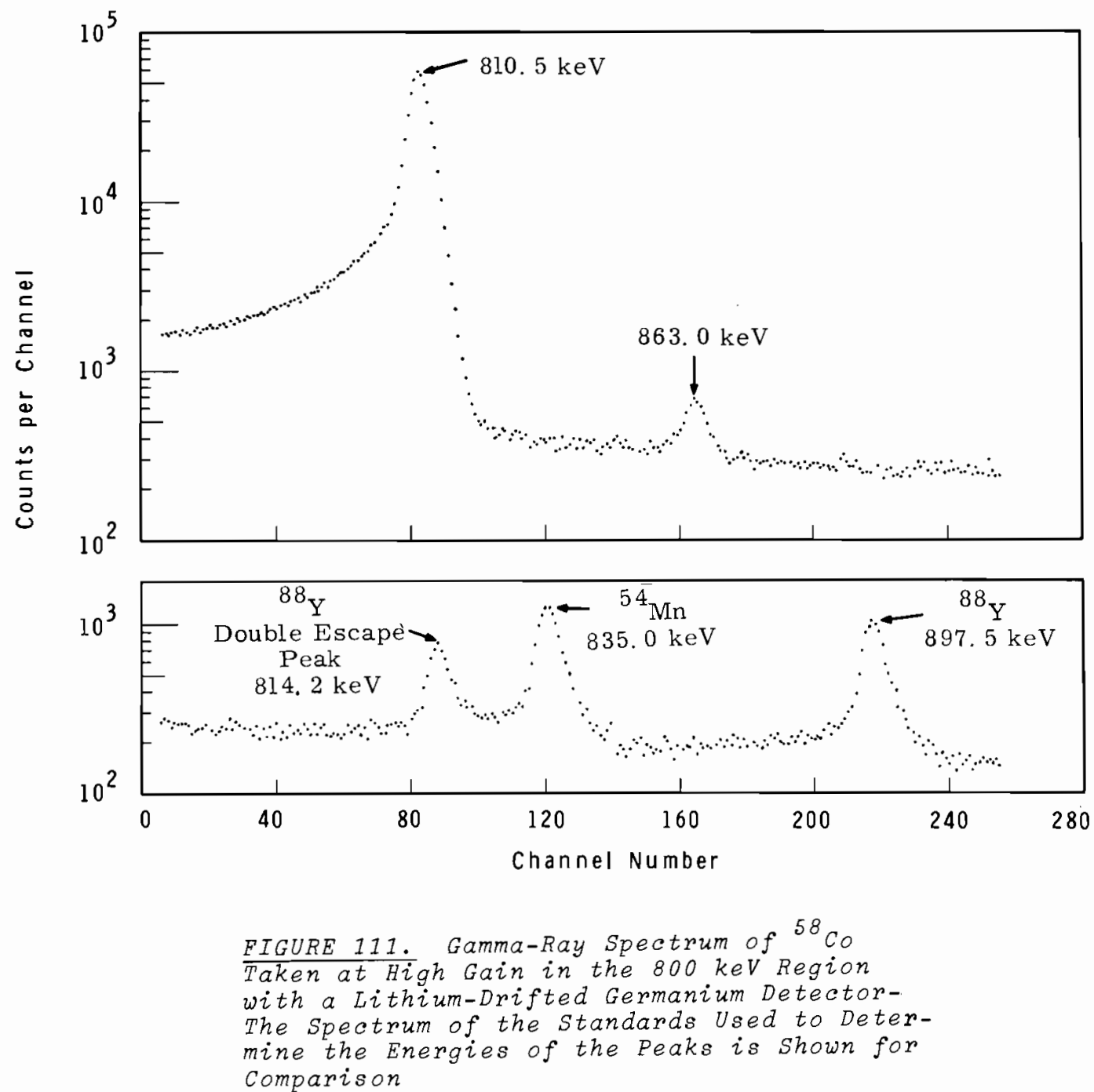

TRACE ELEMENTS IN CANCEROUS AND NONCANCEROUS HUMAN TISSUES-C. W. Thomas

A preliminary study was made of the trace element content of cancerous and noncancerous tissues by measuring the ratio in which these elements occurred 
in normal and cancerous tissues of 1 ung, liver, kidney, and brain. Trace elements were determined by activation analysis using the Hanford reactors and the radionuclides of induced activity were determined without chemical separation using an anticoincidence-shielded multidimensional gamma-ray spectrometer.

The tissues were selected and handled in a manner such as to minimize contamination. Both the normal and cancerous tissues came from the same patient thus eliminating differences in tissue composition from different individuals. To keep contamination at a minimum, samples were handled with plastic forceps and aliquots dissected using plastic knives. The aliquots were encapsulated in vitreous silica ampules and irradiated for various time periods after which the ampules were frozen in liquid nitrogen, opened, and the contents mounted on planchets for counting. By using the multidimensional analyzer no chemical separation steps were necessary to measure the radionuclide daughters of the trace elements.

Radionuclide daughters of zinc, scandium, cobalt, iron, antimony, cesium, copper, potassium, sodium, bromine, and phophorus were determined. The results obtained are presented in Table XLVIII in the form of the ratio of the content of the element in the normal tissue to that in the cancerous tissue.

Generally speaking, the elements zinc, scandium, cobalt, iron, antimony, and possibly copper and cesium were deficient in the cancerous tissues. This same trend was noted by Samsah 1 et a1., (153) in the measurement of trace elements in liver, kidney, and intestine cancerous tissues. Converse$1 y$, the common body electrolytes sodium and potassium were present in excess in the cancerous tissues. Phosphorus appeared to be similar in behavior to the body electrolyte elements, while bromine showed little selectivity toward either type of tissue. Wilms tumor showed the greatest variation from the other cancerous tissue, and this may be a result of the different (teratomacious) character of this tumor.

The author is greatly indebted to Dr. C. W. Westman for supplying the samples for this preliminary study.

TABLE XLVIII. Ratio of Trace Elements in Normal to that in Cancerous Tissue

\begin{tabular}{|c|c|c|c|c|c|}
\hline \multirow[b]{2}{*}{$\begin{array}{l}\text { Isotope } \\
\text { Measured }\end{array}$} & \multicolumn{5}{|c|}{$\frac{\text { Normal }}{\text { Cancerous }}$} \\
\hline & $\begin{array}{l}\text { Bronchogenic } \\
\text { Carcinoma } \\
\text { (Lung) }\end{array}$ & $\begin{array}{l}\text { Squamous Cel1 } \\
\text { Carcinama } \\
\text { (Liver) } \\
\end{array}$ & $\begin{array}{l}\text { Wilns } \\
\text { Tumor } \\
\text { SKidney) } \\
\end{array}$ & $\begin{array}{l}\text { Astrocytoma } \\
\text { Carcinoma } \\
\text { (Brain) } \\
\end{array}$ & $\begin{array}{l}\text { Hepatic } \\
\text { Carcinoma } \\
\text { (Liver) }\end{array}$ \\
\hline $6 s_{2 n}$ & 2.3 & 1.6 & 0.7 & 1.0 & 1.0 \\
\hline${ }^{46} \mathrm{Sc}$ & 4.5 & 4.2 & 0.4 & 2.0 & 1.5 \\
\hline${ }^{60} \mathrm{Co}$ & 2.4 & 4.0 & 2.0 & 2.5 & 1.6 \\
\hline${ }^{59} \mathrm{Fe}$ & 3.4 & 2.1 & 0.6 & 0.7 & 1.3 \\
\hline $124_{S b}$ & 3.7 & 1.0 & 0.1 & 2.0 & 2.0 \\
\hline${ }^{134} \mathrm{Cs}$ & 3.0 & 1.7 & 0.2 & 1.0 & 1.4 \\
\hline${ }^{64} \mathrm{Cu}$ & 0.8 & 1.0 & 1.2 & 1.5 & 0.9 \\
\hline $42_{k}$ & 0.6 & 0.6 & 0.8 & 0.8 & 0.7 \\
\hline${ }^{24} \mathrm{Na}$ & 0.5 & 0.6 & 0.8 & 0.7 & 1.0 \\
\hline $82_{B \mathrm{r}}$ & 1.0 & 1.0 & 1.4 & 1.0 & 1.1 \\
\hline $322_{p}$ & 0.4 & 0.7 & 1.5 & 1.7 & 0.7 \\
\hline
\end{tabular}




\section{REFERENCES}

1. W. C. Roesch, "East-Pulse Averaging", Hanford Radiological Sciences Research and Development Annual Report for 1964, BNWL-36 2, (1965).

2.W. E. Wilson and W. C. Roesch, "East-Pulse Averaging," this report. $p \cdot 1$

3. H.H. Rossi, "Microscopic Energy Distribution in Irradiated Matter," Chapter for Second Edition of Radiation Dosimetry, to be pubiished.

4. W. C. Kaiser, and J.A.M deVizliers, "Relative Light output Evaluation of Different Commercial Plastic Scintilzators," IEEE Transactions on Nuclear Science, NS-11, P 29-37, (1964).

5. D. M. Fleming and N. E. Mutka, "A Pulsed X-Ray Machine," Radiological Physics Annual Report, BNWL-36, p. 2.52, (1964)

6. K. G. Zimmer, Studies on Quantitative Radiation Biology, Hafner Publishing Co., Inc., New York, 1961 .

7. W. C. Roesch, "Locaz-EnergyDensity Distributions," this report, p. 18

8. H. H. Rossi, M. H. Biavati, and W. Gross, "Local Energy Density in Irradiated Tissues," Radiation Research, Vol. 15, pp. 437-439, (1961).

9. L. L. Nichols, "The Secular Change of Pu-Be Neutron Sources," Hanford Radiological Sciences Research and Development Annual Report for 1964,

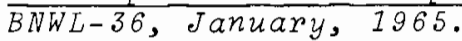

10. J. DePangher, "Radiation Effects in $B F_{3}$ Tubes," Research and DeveLopment Activities in the Radiological Sciences, $H W-73337, p p .48-$ 51, Jan.-Dec., 1961.

11. J. DePangher, "A Reproducible Precision Polyethylene Long Counter for Measuring Fast-Neutron Flux," Research and Development Activities in the Radiological sciences, HW-70050, pp.51-57, January, 1961.
12. H. H. Rossi, Radiation Dosimetry, Academic Press, second edition, in press.

13. B. J. Biavati, "Dose-Effect Curves and some Mathematical Models," Trans. N.Y. Acad. Sci. Vol. 34, pp. 551-561, (1965).

14. W. Swietoslawski, Reinhold Publishing Corp., (1964)

15. U. Eano, "Note on the Bragg-Gray Cavity Principle for Measuring Energy Dissipation," Radiation Research, Vol. 1, pp. $\frac{2}{37-240}$, (1954).

16. G. Faizza, "The Flux of Secondary Ionizing Particles in a Uniformly Irradiated Homogeneous Medium of Varying Density: Application to Walzed Ionization Chambers," Radiation Research, Vol. 4, pp. 102-109, (1956).

17. H. H. Rossi and Walter Rosenzweig, "A Device for the Measurement of Dose as a Function of Specific Ionization," Radiology, Vol. 64, pp. 404-411, (1955).

18. H. H. Rossi, "Specification of Radiation Quality," Radiation Research, Vol. 10, pp. 522-531, (1959).

19. M. H. Biavati, E. Boer, and H. H. Rossi, "Tissue Equivalent Proportional Counter and Gas Flow System," Annual Report of Research Project, NYO-10451, January 1963 .

20. M. H. Biavati, W. Rosenzweig, H. H. Rossi, and I. Miyanaga, "The Dependence of $R B E$ on the Energy of Fast Neutrons III, Evaluation of Radiation Quality," Radiation

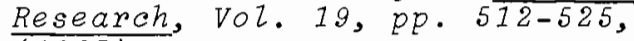
(1963).

21. W. A. Glass and D. N. Samsky. "Specific Ionization of Monoenergetic Protons in Tissue Equivalent Gas," this report, p. 5

22. L. A. Braby, "Tissue Equivalent Ion Chamber Development," this report, p.27

23. F. R. Shonka, J. E. Rose, and G. Eaizza, "Geneva Conference Paper," Progr. Nucl. Energy Ser. XIII, 753,1958 . 
24. L. A. Braby, "Fulse Reader for Ion Chambers," Hanford Radiological Sciences Research and Development Annual Report for $1964, B N W L-3 \bar{E}$, p. 46,46 (1964).

25. H. H. Rossi and Walter Rosenzweig, "Measurements of Neutron Dose as a Function of Linear Energy Transfer," Radiation Research, Vol. 2, pp. 417-425, (1955).

26. Randalz S. Caswell, "Deposition of Energy by Neutrons in spherical Cavities," to be published in Radiation Research.

27. W. A. GZass, "Low Energy Fast Neutron spectrometer," to be pubiished.

28. D. C. Lehfeldt, Ceramics Research and Deve Zopment Quarterly Report, Juzy-September, 1965, BNWL-

29. R. Loevinger, J. G. Holt, and G. J. Hine, in G. H. Hine and G. L. Brownell, Radiation Dosimetry, Academic Press, New York $(1956), p .824$.

30. F. W. Spiers, in G. J. Hine and G. L. Brownelz, Radiation Dosimetry, Academic Press, New York $(1956)$, p. 24.

31. H. E. Johns and J. S. Laughlin, in G. J. Hine and G. L. Brownelz, Radiation Dosimetry, Academic Press, New York (1956), p. 104.

32. G. N. Whyte, Principles of Radiation Dosimetry, John Wiley and Sons Inc., New York (1959), p. 18.

33. M. J. Berger and S. M. Seltzer, Tables of Energy Losses and Ranges of Electrons and Positrons, NASA $S P=3012,(1964)$.

34. K. L. Swinth and B. I. Griffin, "Counting Plutonium in Animals and ir Excreta," Hanford Radiological Sciences Research and Development Annual Report for $1 \overline{9} 64, B N W L-36$, January, 1965 .

35. B. Altshuler, and B. Pasternack, Health Physics, Vol. 9, pP. $293-$ $298,(1963)$.
36. B. I. Griffin and W. C. Roesch, "A Scintilzation Counter for In Vivo Detection of Plutonium," Hanford Radiological Sciences Research and Development Annual Report for 1963, HW-81746, January 1964 .

37. K. L. Swinth and B. I. Griffin, "Progress in Plutonium whole Body Counting," Hanford Radiological Sciences Research and Development Annual Report for 1964, BNWL-36, January, 1965 .

38. E. A. Putzier, C. R. Lagerquist, S. E. Hammond, J. R. Mann, and C. R. Piltingsrud, Evaluation and Treatment of an Acute Internal Exposure to Plutonium, RFP-446, $(1965)$.

39. Rama, Minoru Kiode, and Edward $D$. Goldberg, Nature, Vol. 191, p. 162 , (1961).

40. A. H. Seymour, Gamma-Emitting Radionuclides in Tuna samples from the Tokyo Central Fish Market, 1962, University of Washington, Laboratory of Radiation Biology, DOC. U.W.F.L. -90 .

41. T. R. Folsom, Observations Related to Falzout in the Pacific ocean in 1964, University of California, Scripps Institute of Oceanography, Progress Report SIO, pp. 71, 1964-A $(1965)$.

42. H. E. Palmer and T. M. Beasley, Scierce, Vol. 149, pp. 431-432, (1965).

43. T. R. Folsom and D. R. Young, Nature, Vol. 206, pp. 804-805, (1965).

44. H. E. Palmer, W. C. Hanson, B. I. Griffin, and L. A. Braby, Science, vol. 147, pp. 620-621, (1965).

45. International Committee Radiological Protection, Recommendations: Report 2, Permissible Dose of Internal Radiation, (Pergamon, New York, 1960).

46. W. C. Hanson, H. E37Palmer, "Seasonal cycle of $\mathrm{Cs}^{23}$ in some Alaskan Natives and Animals," Health Physics, Vol. 11, (1964). 
47. H. E. Palmer, "Simplified whole Body Counting," Hanford Radiological Sciences Research and Development Annual Report for 1964, BNWL-36, January, 1965.

48. H. E. Palmer, "Simplified whole Body Counting," Health Physics, (in press).

49. C. C. Lushbaugh, N. J. Basmann and D. B. Hale, "Electronic Measurement of Celzular Volumes. VI. Electronic Improvement of Coulter Counter Resolution," $L A M S-3034, p p .261-265$, July 1962June 1963 .

50. C. C. Lushbaugh and D. B. Hale, "Electronic Measurement of Celzular Volume. VII. Biologic Evidence for Two Volumetrically Distinct Subpopulations of Red Celz," $L A M S-3034, p p .270-278$.

51. H. E. Kubitschek, "Apertures for Coulter Counters," Rev. Sci. Instr, Vol. 35, p. 1598, (1964).

52. C. C. Lushbaugh, E. C. Anderson, $H$. I. Israel, D. B. Hale and N. J. Basmann, "Electronic Measurement of Celzuzar Volume. V. Changes in Red Blood Cells Resulting from Nonphysiologic pH," LAMS-3034, pp. 253-260, July 1962-June 1963.

53. D. R. Kalkwarf and R. W. Henkens, "Chemical Protection of Red Blood Cezzs from Radiation Damage," HW-73337, January 15, 1962.

54. D. R. Kalkwarf and R. W. Henkens, "Kinetics of Radiation-Induced Hemolysis of Human Red Blood Cells," $H W-77609$, pP. 3.82-3.84, January 1963 .

55. W. D. Felix, "Kinetics of Diffusion Through Human Erythrocyte Membranes," BNWL-36, Vol. III, pp. 3.21-3.31, January, 1965.

56. K. S. Trincher and A. M. Kuzin, "Significance of Water in Radiation Damage to the Erythrocytes," AEC-tr-6404, pp. 48-54, (TransZation of Radiobiologiya, Vol. IV, No. 1, pp. 36-40, 1964).

57. M. Anbar and J. K. Thomas, "Pulse Radiolysis Studies of Aqueous Sodium Chloride Solutions," J. Phys. Chem., Vol. 68, pp. 3829-3835, (1964).
58. J. F. Ward and L. S. Myers, Jr., "The Effect of Chloride Ions on Some Radiation Chemical Reactions in Aqueous Solutions," Rad. Res., Vol. 26, PP. 483-492, (1965).

59. A. 0. Alzen, The Radiation Chemistry of water and Aqueous Solutions, p. 60 ff., Princeton: D. Von Nostrand, (1961).

60. D. R. Kalkwarf, "Effect of Temperature on Radical-Capturing Reactions in Irradiated Aqueous Solutions," $H W-S A-3063$, (1963).

61. Microwave Associates type MA $4610 \mathrm{~A}$ in Ma $4611 \mathrm{~A}$ matching holder is the only type tested. Similar items are also available from PhilcoLansdale.

62. R. 0. Wright, "The Backward DiodeWhen and How to Use It," Microwaves, December, 1964.

63. B. I. Bleany and B. Bleany, "Electricity and Magnetism," oxford University Press, London, Section 17.5, 1959 .

64. H. O. Sorensen, "Using the Hot Carrier Diode as a Detector," Hewlett-Packard Journal, Vol. 17:4, pp. 2-5, December, 1965 .

65. G. Feher, Bell Syst. Tech. J., Vol. 36, pp.449-484, (1957).

66. Varian Associates, Tech. Information Bulz., Fall 1965.

67. G. K. Fraenkel, Fhysical Methods of Organic Chemistry, Chap. XLII, 3rd. Edition, Interscience Publ. N.Y., (1960).

68. R. N. Diebel and D. R. Kalkwarf, Hanford Radiological Sciences Research and Development Annual Report for 1963, $H W-81746$, pp.3.113-3.119, January, 1964.

69. L. Michaelis, M. P. Schulbert and S. Granick, "The Free Radicals of the Type of Wursters Salts," J. Amer. Chem. Soc., Vol. 61, pp.1981-1992, (1939).

70. J.R. Bolton, A. Carrington and J. Dos Santos-Veiga, "Analysis of High Resolution ESR Spectra. A Reinterpretation of the Wursters Blue Ion Spectrum," Mol. Phys., Vol. 5, pp. 615-619, (1962). 
71. K. H. Hausser, "Reinterpretation of the Wurster's Blue E.S.R. Spectrum," Mol. Phys., Vol. 7, p. 76, (1964).

72. A. Ehrenberg, "Detailed ESR Spectra of the Free Radicals of FMN and Fad," Acta Chem. Scan., Vol. 14, pp. 766-767, (1960).

73. A. V. Guzzo and G. Tolzin, "Electron Paramagnetic Resonance Studies of Riboflavin and Its Derivatives. 1. The Isoalloxazine Semiquinones in Acid," Arch. Biochem. Biophys., Voz. 103 , pp. 203-243, (1963).

74. A. Ehrenberg, Electronic Aspects of Biochemistry, edited by $B$. Puliman, Academic Press, New York, $1964, p .382$.

75. D. C. Borg and G. C. Cotzias, "Interaction of Trace Metals with Phenothiazine Drug Derivatives, III. Theoretical Part," Proc. Nat. Acad. Sci., Vol. 48, pp. $\overline{643-652,}$ $(1962)$.

76. P. B. Ayscough and C. Thomson, "Electron-Spin Resonance observations on the Reduction of Methylene Blue and Related Compounds," J. Chem. Soc., pp. 2055$2060,(1962)$.

77. D. R. Kalkwarf and R. N. Diebel, "Comparison of Radicals Fromed by Gamma Irradiation and Dithionite Reduction of Aromatic NitroCompounds in Water," Hanjora Radiological Sciences Research and Development Annual Report for 1964 BNWL-36 III, pp. 3.12-3.20, (January, 1965 ).

78. D. R. Kalkwarf and R. N. Diebel, "Long-lived Organic Radicals Formed in Irradiated Aqueous Solution," Hanford Radiological Sciences Research and Development Annual Report for $1965, \overline{B N W L-}$

79. W. Kohnlein and A. Mulzer, "Absolute Yiezd Measurements of Radiation-Produced Radicals by ESR," Phys. Med. Biol., Vol. 6, pp. 599-604, (1964).

80. J. A. Friend and N. K. Roberts, "A Polarographic Study of Some Wursters Salts," Australian J. Chem., Vol. 11, pp.104-119, (1958).
81. A. Henglein, $M$. Boysen, and $W$. Schnaber, "Die Zersetzun des Polymethyl Acryzsauremethylesters in Losung unter deam Einfluss von 60 Co-Gamma-Strahlen," Leit. Phys. Chem. N.F., Vol. 10, pp.137-155, (1957).

82. P. B. Ayscough and C. Thomson, "Electron-Spin Resonance Observations on the Reduction of Methylene Blue and Related Compounds," J. Chem.Soc.2.pp. 2055-2060, (1962).

83. D. R. Kalkwarf, "Radiation Protection of Agarose Jellies," Hanford Radiological Sciences Research and Development Annual Report for 1964, BNWL-36 III, p. 3.4, January, 1365.

95. T. M. Beasley. Hanford Radiological Sciences Research and Develpment Report for 1964, BNWL-36, pp. 3.55-3.57. 1965 .

86. R. D. Cherry. "Alpha-Radioactivity of Plankton," Nature: vol. 203, pp. 139-143. 1964.

87. T. M. Beasley. "Application of Tertiary Amine Extraction to the Determination of Uranium in Biological Materials," Health Physics, vol. 11, pp. 1059-10大5. 1965.

88. Stuart C. Black. "Low-Level Polonium and Radiolead Analysis," Health Physics, vol. 7, pp. 87-91. 1961 .

89. M. Koide Rama and E. D. Goldgerg. "Lead-210 in Natural Waters," Science, vol. 134, pp. 98-99. 1961 .

90. R. B. Holtzman. "Lead 210 (RaD) and Polonium-210 (RaF) in Potable Waters in Illinois," The Natural Radiation Environment, pp. 321. University of Chicago Press, Chicago, Ilzinois, 1964.

91. C. R. Hizl. "Po"10: a Naturalzy occurring Component of "Ealz-Out'," Symposium on Radioactivity in Scandinavia, Denmark. Oet., 1964.

92. R. G. Geier. Reactor Effluent Research and Development Program. HW-81063, Richland, Washinaton. February 28, 1964. 
93. R. F. Foster. Evaluation of Radiological Conditions in the Vicinity of Hanford for 1964, BNWL-90. Juzy 1965 .

94. R. W. Perkins. Source of Reactor Effluent Water Radioisotopes, HW-69969, Richland, Washington. May 1961. (Secret)

95. D. E. Robertson and R. W. Perkins. Reduction of Effluent Water Radionuclides by the Addition of Sodium Silicate to Process Water, HW-75782, Richland, Washington. December 10, 1962 .

96. R. W. Perkins and D. E. Robertson. The Effect of Chemical Additives and Coating Materials on the Adsorption of Radionuclide Parent Elements on Aluminum Surfaces, HW-75976, Richland, Washington. December $26,1963$.

97. D. E. Robertson and R. W. Perkins. Reduction of Radionuclides in Reactor Effiuent Water: Final Report on the Effect of Chemical Additives and Coating Material on the Adsorption of Radionuclide Parent Elements in Process Water on Aluminum Surfaces, $H W-80557$, Richland, Washington. December 23 , 1963.

98. R. W. Perkins and D. E. Robertson. "Selective and Sensitive Analysis of Activation Products by Multidimensional Gamma-Ray Spectrometry," 1965 International Conference, Modern Trends in Activation Analysis, College Station, Texas. April 19-22, 1965.

99. R. G. Geier. "Interim Report I, Production Test IP-728, Half Plant Sodium Silicate Test," RL-REA2546. General Electric Co., Richland, Washington. September 16 , 1965. (SECRET)

106. D. E. Robertson. Evaluation of Selected Procedures for the Separation of Radioarsenic from Reactor Effluent Water, HW-76379, Richland, Washington. March 1963.

101. R. W. Perkins, J. L. Nelson, and W. L. Haushild. "Behavior and Transport of Radionuclides in the Columbia River Between Hanford and Vancouver, Washington," oceanography and Limnology. In Press. (1966)
102. J. L. Nelson, R. W. Perkins, and J. M. Nielsen. Progress in Studies of Radionuclides in Columbia River Sediments, HW-83614, General Electric Company, Richland, Washington. December, 1964 .

103. R. W. Perkins. "An Anticoincidence-Shielded Multidimensional Analyzer," Nuclear Instruments and Methods, vol. 33, pp. 71-76. 1965 .

104. J. L. Nelson. "Scouring of Radionuclides Deposited with Columbia River sediments During the Spring Freshet," Radiological Chemistry, Hanford Radiological Sciences Research and Development Annual Report for 1964, BNWL-36 III, pp3.71-3.74. 1965 .

105. David R. Schink and James T. CorZiss, ed. "Occasional Publication No. 3," Symposium on Marine Geochemistry, Narragansett Marine Laboratory, University of Phode Is land. 1965 .

106. Julian M. Nielsen. Behavior of Radionuclides in the Columbia River, PID-7664, pp.91-112. 1963.

107. R. W. Perkins, J. M. Nielsen. "Zine-65 in Foods and People", science, vol. 129 , pp. 94-95. $\overline{1959}$.

108. R. W. Perkins, J. M. Nielsen, W. C. Roesch, and R. C. MeCalz. "Zine-65 and Chromium-51 in Foods and People", Science, vol. 132, pp. $1895-1897.1960$.

109. R. W. Perkins and J. M. Nielsen. "Sodium-22 and Caesium-134 in Foods, Man, and Air," Nature, vol. 205, pp. 866-867. 1965 .

110. R. W. Perkins. "The Direct Measurement of Radionuclides in BioAssay Samples by Multidimensional Gemma-Ray Spectrometry," Proceedings of the 10th Annual BioAssay and Analytical Chemistry Meeting, p.190. Cincinnati, Ohio, oct. 8-9. 1964.

111. International Committee on Radiological Protection. "Report of Committee II on Permissibile Dose for Internal Emmitters." Health Physics, vol. 3, Publication NO. 2, 1959, ICRP. 1960. 
112. M. Eisenbud, et al. "Naturally occurring Radionuclides in Foods and Waters from the Brazilian Areas of High Radioactivity," The Natural Radiation Environment. J. A. S. Adams and W. M. Lowder, Ed., University of Chicago Press, 1964.

113. H. E. Palmer, W. C. Hanson, B. I. Griffin, and $W$. C. Roesch. "Cesium-137 in Alaskan Eskimos," Science, vol 142, pp. 64-65. 1963.

114. H. E. Palmer and T. M. Beasley. "Iron-5.5 in Humans and Their Food," Science, vor. 149, pp. $4 3 1 - 4 3 2 \longdiv { 1 9 6 5 }$.

115. R. B. Holtzman. "Measurement of the Natural Contents of RaD (PB210) and RaF (Po210) in Human Bone--Estimates of Whole Body Burdens," Health Physics, vol 9, pp. 385-400. 1963 .

116. R. B. Holtzman. "Natural Content of $R A D$ ( $P b^{2} 10$ ) and $R A F$ (PO210) in the Human Body", Radioactivity in Man, pp. 433-442, C. R. Meneely and S. M. Linde, Ed. Charles C. Thomas, Publisher, Springfield, Illinois.

117. R. B. Holtzman. "The Source of Unsupported 210 Po (RaF) in Tissue," Health Physics, vol 10, pp. 763-4. 1964 .

118. R. B. Holtzman. "210 $\mathrm{Pb}$ (RaD) in Inhabitants of a Caribbean Is Land", Health Physics, vol 11, pp. 477480,1965 .

119. R. B. Holtzman. ANL-6?69, pp. 5965. July $1962-63$.

120. C. W. Silz and C. Wilzis. "Radiochemical Determination of Lead-210 in Mill Products and Biological Materials," Anal. Chem. vol. 37, pp. 1661-1671. 1965.

121. T. M. Beasley. Unpublished data.

122. W. C. Hanson and H. E. Palmer. "The accumulation of Falzout Cesium-137 in Northern AZaskan Natives," Trans. North Amer. Wizdlife and Natural Resources Conference, vol $29, p p \cdot 215-225$. 1964 .
123. H. E. Palmer, W. C. Hanson, B. I. Griffin, and L. A. Braby. "Radioactivity Measured in Alaskan llatives, 1962-1964", Science, vol. 147 , pp. $620-621.196 \overline{5}$.

124. M. Sultzer and J. B. Hursh. "Polonium in Urine of Miners Exposed to Radon". Arch. Ind. Hyg. \& Occ. Med., vol. 9, pp. 8D-99. 1954.

125. R. M. Fink, Ed. "Biological Studies with Polonium, Radium, and Piutonium", National Nuclear Energy Series, pp. 1-53. New York, McGraw-Hiz Book Company, Inc. 1950.

126. R. W. Perkins, J. M. Nielsen, and C. W. Thomas. "Air Concentrations of Twelve Radionuclides from 1962 through Miä-1964," Science vol. 146, pp. 762-764. 1964.

127. T. R. Folsom and D. R. Young. "Silver-110m and Cobalt-60 in oceanic and coastal organisms," Nature, vol. 206, pp. 803-805. 1965 .

128. Edward D. Goldberg. Amn. Rev. Phys. Chem., vol. 12, pp. $\overline{29-48.1961 .}$

129. C \& E News, 42, No. 22, pp. 1A-48A, Special Reprot, "Chemistry and the oceans."

130. J. Mauchline and w. L. Templeton. oceanogr. Mar. Biol. Ann. Rev. vol 2, pp. 229-279. 1964.

131. Donald F. Schutz. Geochemistry Technical Report No. 9, TID-21067, Yale univ. Hew Haven, Conn. Apriz, 1964.

132. D. Lal, J. R. Amold and B. L. K. Somayajulu. Geochimica et Cosmochimica Acta 28, pp.1111. 1964.

133. R. W. Perkins, C. W. Thomas, and J. M. Nielsen. "Airborne Radionuclide Measurements and Physical Characteristics Determination," Proceedings of the 2nd AEC Fallout Conference, Germantown, Maryland, November 3-6. 1964.

134. C. W. Thomas. "Physical and Chemical Characteristics of Various Fallout Radionuclides," III Radiological Chemistry Hanfor $\bar{d}$ Radiological Sciences Research and Development Annual Report for 1964. BNWL-36 III, pp. 3.58-3.62. 1965 
135. E. P. Hardy, Jr., and W. R. Collins, 144. Jr. Health and Safety Laboratory, Fallout Program Quarterly Summary Report for June, 1963 to September 1, 1963. USAEC Report HASL-140. october 1, 1963 .

136. C. W. Thomas. "Air Concentrations of Short Lived Radionuclides Resulting from the 1964 Chinese Detonations," BNWL-36 III, pp. 3.62-3.65. January 1965.

137. J. D. Ludwick. "Investigation of Inert Atmospheric Tracers; Xe133," Radiological Chemistry, Hanford Radiological Sciences Research and Development Annual Report for 1964, BNWL-36 III, pp.3.104-3.114. January 1965.

138. E. C. Watson and C. C. Gamertsfelder. Environmental Radioactive Contamination As A Factor In Nuclear Plant Siting Criteria, HWSA-2809. March 29, 2963, REV. Apriz 16, 1965 .

139. J. D. Ludwick. "Atmospheric Diffusion studies with Fluor escein and Zine Sulfide Particles As Dual Tracers," J. Geophysical Research. Scheduled for publication March 15, 1966.

140. J. D. Ludwick. "Investigation of the Nature of I131 in the Atmosphere," Hanford Radiological Sciences Research and Develop ment Annual Report For 1963 . HW-81746, pp. 3.58-3.68. January, 1964 .

141. R. E. Brown and D. J. Brown. "Problems Associated with the Extension of the Stratigraphic Units of South-Central Washington, "BNSA-135. 1966 .

142. A. C. Waters. "Stratigraphic and Lighlogic Variations in the Columbia River Basalt," Am. Jour. Sci., Vol 257, pp. 583-561. 1961.

143. H. U. Schmincke. "Petrology, Current Directions and History of the Upper Lake Yakima Basalt Flows and Interbedded Sediments, South-Central Washington," Fourth Annual Grad. Student Symposium in the Geol. Sei., University of California, Los Angeles, California, April 17-18, 1964, pp 1415.1964 .
F. A. Frey and L. Haskins. "Rare Earths in oceanic Basalts," $J$. Geophys. Res., vol 69, pp. $7 \overline{75}$ 780.1964.

145. R. W. Perkins and J. M. Nielsen. "Multidimensional Gamma-Ray Spectrometry and Its Use in Health Physics," to be published in the Proceedings of the Annual Meeting of Health Physics Society, Los Angeles, California, June 14-17, 1965 .

146. R. W. Perkins and D. E. Robertson. "Multidimensional Gamma-Ray Speetrometry and Its Application in Radiochemistry," to be published in Proceedings of National Meeting of American Chemical Society, Detroit, Michigan. April, 1965.

147. M. W. Hizl. "An Anticoincidence Shiezded Ge (Li) Gamma-Ray Spectrometer," vol. 36, No. 2 of Nuclear Instruments and Methods. 1965 .

148. J. M. Cork, M. K. Brice, and L. C. Schmid. Phys.Rev., vol $99, p p .703$. $19 \overline{55}$

149. H. Frauenfelder, N. Levine, $A$. Rossi, and S. Singer. Phys. Rev. vol. 103, pP. 352. 1956 .

150. C. S. Cook, F. M. Tomnovec. Phys. Rev., vol 104, pp. 1407. 1956 .

151. D. MacArthur, R. Goodman, A. Artna, and $M$. J. Johns. Nuclear Physics, vol. 38, pp. 106. 1962 .

152. S. Malmskog. Nuclear Physics, vol. 51, pp. 690. 1964.

153. K. Samsahl, D. Brune, and P. 0. Wester. "Simultaneous Determination of 30 Trace Elements in Cancerous and Non-cancerous Human Tissue Samples by Heutron Activation Analysis," Intern. J. Appl. Radiation Isotopes, vol. 16, pp. 273-281. Apriz, 1965. 


\section{DISTRIBUTION}

Number of Copies

\begin{tabular}{|c|c|}
\hline 272 & $\frac{\text { Division of Technical }}{\text { Information Extension }}$ \\
\hline 1 & $\begin{array}{l}\text { AIF Task Force } \\
\text { MPR Associates, Inc. } \\
815 \text { Connecticut Ave. NW } \\
\text { Washington D.C. } 20006\end{array}$ \\
\hline 1 & $\begin{array}{l}\text { Air Force Cambridge } \\
\frac{\text { Research Laboratory }}{\text { Bellford, Massachusetts }} \\
\text { D. A. Hargen }\end{array}$ \\
\hline 1 & $\begin{array}{l}\text { Atomic Energy of Canada } \\
\text { Limited } \\
\text { Chalk River, Ontario } \\
\text { C. A. Mawson }\end{array}$ \\
\hline 2 & $\begin{array}{l}\text { Atomic Energy Estab- } \\
\frac{\text { lishment Trombay }}{\text { Bombay 73, India }} \\
\text { P. N. Krishnamoorthy } \\
\text { K. T. Thomas }\end{array}$ \\
\hline 2 & $\begin{array}{l}\text { Atomic Energy Research } \\
\text { Establishment } \\
\text { Harwell, Berks, England } \\
\text { R. H. Burns } \\
\text { E. Glueckauf }\end{array}$ \\
\hline
\end{tabular}

1 Australian A.E.C.

1

1

Number of Copies Post Office Coogee New South Wales, Australia

A. W. R. Wilson

Brookhaven National L. P. Hatch

Center for Nuclear Studies

P.0. Box 6, Fontenayaux-Roses

(Seine) France

$$
\text { A. de Calmes }
$$

Center for Nuclear

Studies

P.0.Box 2, Saclay

Gif-sur-Yvette ( $S \& O)$, France

F. Duhame 1

A. Menoux

Douglas United Nuclear

T. W. Ambrose

P. C. Jerman
2

1

duPont Company

Aiken, South Carolina

W. B. Scott

duPont Company Wilmington, Delawa re

V. R. Thayer

ENEA (OECD) Health and Safety Office

38, Blvd. Suchet, Paris XVI, France

E. Wallauschek

Eurochemic Library Mol, Belgium

General Electric Company M. C. Leverett

R. T. Trumbul1

General Electric Company Advanced Technology Laboratories 1 River Road Schenectady. 5, New York J. W. Hea $1 y$-Consultant Technica 1 Hazards

Geological Survey of the United Kingdom Water Division Stevenson Buchan, Chief Geologist

Geological Survey of the United States Washington 25, D.C. M. King Hubbert C. V. Theis

George Washington University

Washington, D.C.

C. R. Naeser

Geotechnical Corporation

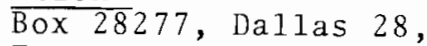
Texas

W. B. Heroy

1

Geselschaft Kernforschung $\mathrm{mbH}$ Karlsrike 5 West Germany Dr. H. Krause 
DISTRIBUTION (contd)

Number of Copies

2

3

1

2

2

2

9

$$
\frac{\text { Hanford Occupational }}{\frac{\text { Health Foundation }}{\text { W. D. Norwood }}}
$$

International Atomic
Energy Agency
Vienna l, Kaerntner-
ring ll, Austria
H. Seligman
J. Servant

Isochem, Inc.

R. E. Tomlinson

F. $\mathrm{Hi} 11$

D. J. Brown

Johns Hopkins University

Baltimore, Maryland W. A. Patrick

$\frac{\text { Lawrence Radiation }}{\text { Laboratory }}$

G. H. Higgins

J. B. Knox

National Institute of

Radiological Sciences 250 , Kurosuna-Cho, Chiba-shi, Japan

M. Saiki

M. Suzuki

N. V. Belchim

200 Boeretang

Mol, Belgium

Paul Dejonghe

Leo $H$. Baets 1 e

Richland Operations

Office

J. T. Christy

J. P. Derouin

P. G. Holsted

C. L. Robinson

R. K. Sharp (2)

Technical Informa-

tion Library (2)

R. D. Wildman

Oak Ridge National Laboratory

K. Z. Morgan

E. G. Struxness

Number of Copies

1

1

1

1

1

9

1

1

\author{
University of Arizona \\ Tucson, Arizona \\ Department of Geology \\ E. S. Simpson \\ University of Califor- \\ nia \\ Berkeley, California \\ Department of Civil \\ Engineering \\ W. J. Kaufman \\ University of I11i- \\ nois

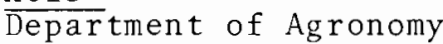 \\ M. B. Russe 11 \\ University of North \\ Carolina \\ Chape1 Hill, N.C. \\ Department of Chem- \\ istry \\ H. C. Thomas
}

University of Wisconsin Department of Chemistry

H. Gladys Swope

U.S. Atomic Energy Commission

Washington, D.C.

Division of Biology

and Medicine

N. F. Barr

H. D. Bruner

W. D. Claus

C. L. Dunham, Dir.

H. Hollister

A. W. Klement

S. A. Lough

J. R. Totter

J. N. Wolfe

Division of Naval

Reactors

R. S. Brodski

Division of production

W. L. Lennemann

Division of Reactor

Development

W. G. Belter

Health and Safety Laboratory

New York City

John Harley, Dir. 


\section{DISTRIBUTION (contd)}

Number of Copies

1

1

1

216
U.S. Atomic Energy

Commission Savannah

River Operations Office

K. K. Brown

U.S. Pub1ic Health

Service

Division of Health

Mobilization

Washington, D.C.

$\mathrm{J}$. J . Lang, Re-

search Branch

World Health Organization

Geneva, Switzerland

R. L. Dobson, M. D.

Batte11e-Northwest

G. J. A1kire

F. W. Albaugh

W. J. Bair

C. A. Bennett

R. J. Brouns

L. J. Chockie

W. J. Clarke

R. F. Dickerson

R. L. Dillon(2)

W. L. Dotson

C. E. Elderkin

R. J. Englemann

S. L. Fawcett

E. H. Finney

R. F. Foster

P. A. Fuqua

J. J. Fuquay (50)

J. K. Green (5)
Number of Copies

W. A. Haney

J. F. Honstead

F. P. Hungate

E. R. Irish

R. T. Jaske

R. L. Junkins

D. R. Kalkwarf

A. R. Keene

H. A. Kornberg

C. R. Lagergren

K. H. Larson

R. E. Nakatani

C. E. Newton

J. M. Nielsen (100)

R. F. Palmer

H. M. Parker

R. S. Pau 1

D. W. Pearce

R. W. Perkins

I. C. Roberts

W. C. Roesch (6)

L. C. Schwendiman

W. G. Spear

C. L. Simpson

A. J. Stevens

M. F. Sullivan

R. C. Thompson

C. R. Tipton, Jr.

E. E. Voiland

M. T. Walling

W. E. Wilson

D. C. Worlton

Biology Library(2)

Technical Informa-

tion Files(5)

Technical Publications (2) 